Solução numérica do modelo PTT para escoamentos viscoelásticos com superfícies livres 



\title{
Solução numérica do modelo PTT para escoamentos viscoelásticos com superfícies livres
}

\author{
Gilcilene Sanchez de Paulo
}

Orientador: Prof. Dr. Murilo Francisco Tomé

Tese apresentada ao Instituto de Ciências Matemáticas e de Computação - ICMC-USP, como parte dos requisitos para obtenção do título de Doutor em Ciências - Ciências de Computação e Matemática Computacional.

USP - São Carlos

Agosto de 2006 

À minha mãe, minha irmã e ao meu pai (in memoriam) 

Agradeço a Deus por mais essa oportunidade em minha vida e pelo amparo em todos os momentos.

Ao meu orientador Prof. Murilo Francisco Tomé pela disponibilidade, compreensão, apoio e amizade durante todo este trabalho.

A todos os professores do grupo de pesquisa do LCAD/ICMC que sempre estiveram presentes auxiliando-me não somente com sugestões para o desenvolvimento do projeto mas também aconselhando-me e incentivando-me a novos desafios.

Aos professores Miguel Nóbrega e Olga Carneiro do Departamento de Engenharia de Polímeros da Universidade do Minho (UM), Guimarães, Portugal, por acolherem-me atenciosamente nesta universidade e também aos professores Fernando Pinho e Manuel Alves da Faculdade de Engenharia da Universidade do Porto, pela oportunidade que deram-me de conhecer vossos ambientes de pesquisa.

Ao professor Pedro Nuno Ferreira Pinto Oliveira do Departamento de Produção e Sistemas da Universidade do Minho, pelo recurso computacional disponibilizado durante o meu estágio de doutorado no exterior.

À minha família que sempre esteve presente em minha vida mesmo com a distância geográfica e, em especial à minha mãe e minha irmã.

Aos amigos da UM e do LCAD, que tanto prezo e respeito, pelo incentivo, idéias e momentos alegres de discontração e, em especial as eternas amigas Dayene e Marcela que fizeram parte do LCAD compartilhando junto a mim a mesma área de pesquisa.

À FAPESP pelo apoio financeiro durante todo o desenvolvimento dessa pesquisa e a CAPES que financiou o meu estágio de doutorado no exterior. 



\section{Resumo}

O objetivo deste trabalho é desenvolver um método numérico capaz de simular escoamentos viscoelásticos com superfícies livres governados pela equação constitutiva não-linear PTT (Phan-Thien-Tanner). Neste trabalho foram apresentados três métodos numéricos para simular escoamentos viscoelásticos modelados pela equação PTT. Dois desses métodos foram desenvolvidos para simular escoamentos viscoelásticos bidimensionais enquanto o terceiro método foi desenvolvido para simular escoamentos viscoelásticos tridimensionais. Estes métodos numéricos foram incorporados aos ambientes de simulação Freeflow2D e Freeflow3D, extendendo estes ambientes para escoamentos viscoelásticos descritos por uma equação constitutiva não-linear. Inicialmente, uma descrição de Freeflow2D e Freeflow3D é apresentada. As equações governantes para escoamentos descritos pelo modelo PTT são dadas na forma de tensorial e as formulações matemáticas para obtenção dos métodos numéricos são apresentadas. As equações que descrevem os métodos numéricos são resolvidas pela técnica de diferenças finitas numa malha deslocada e o fluido é representado por partículas marcadoras usando o método Marker-and-Cell. As condições de contorno para cada tipo de contorno são descritas em detalhes e o cálculo do tensor extra-tensão no contorno rígido é obtido utilizando as idéias de Tomé et al. [84] para o modelo Oldroyd-B. Seguindo a metodologia de Alves et al. [2], a solução analítica do modelo PTT para escoamentos totalmente desenvolvidos em um canal bidimensional é apresentada em detalhes. Esta solução analítica é então usada para validar o método numérico desenvolvido neste trabalho. Os métodos numéricos desenvolvidos nesse trabalho foram aplicados para simular os seguintes problemas: um jato de fluido viscoelástico incidindo numa placa rígida; o inchamento do extrudado e o problema de uma gota esférica de fluido viscoelástico incidindo perpendicularmente contra uma superfície rígida plana.

palavras-chaves: escoamento viscoelástico, modelo PTT, superfície livre, técnica de diferença finita, solução analítica. 



\section{Abstract}

The aim of this work is to develop a numerical method capable of simulating viscoelastic free surface flows governed by the non-linear constitutive equation PTT (Phan-Thien-Tanner). In this work three numerical methods to simulate vicoelastic flows of fluids modelled by the PTT equation are presented. Two of these methods were developed to simulate two-dimensional viscoelastic flows while the third method was developed to simulate three-dimensional viscoelastic flows. These numerical methods were incorporated into the codes Freeflow2D and Freeflow3D, extending these codes to viscoelastic flows described by the non-linear constitutive equation PTT. Initially, a description of Freeflow2D and Freeflow3D is presented. The governing equations for flows described by the PTT model are given in index form and the mathematical formulations for obtaining the numerical methods are presented. The equations describing the numerical methods are solved by the finite difference method on a staggered grid and the fluid is modelled using a Marker-and-Cell type method. The boundary conditions for each type of boundary are described in details and the calculation of the extra-stress tensor on rigid boundaries is performed using the ideas of Tomé et al. [84] for an Oldroyd-B fluid. Following the methodology presented by Alves et al. [2], the analytic solution of the PTT model for fully developed flows in a two-dimensional channel is presented in details. This analytic solution is then used to validate the numerical method developed in this work. Finally, the numerical methods developed in this work were applied to simulate viscoelastic flows such as a viscoelastic jet flowing onto a rigid plate, the extrudate swell of viscoelastic fluids and the simulation of a viscoelastic drop hitting a rigid plate.

keywords: viscoelastic flows, PTT model, free surface, finite difference technique, analytic solution. 



\section{Nomenclatura}

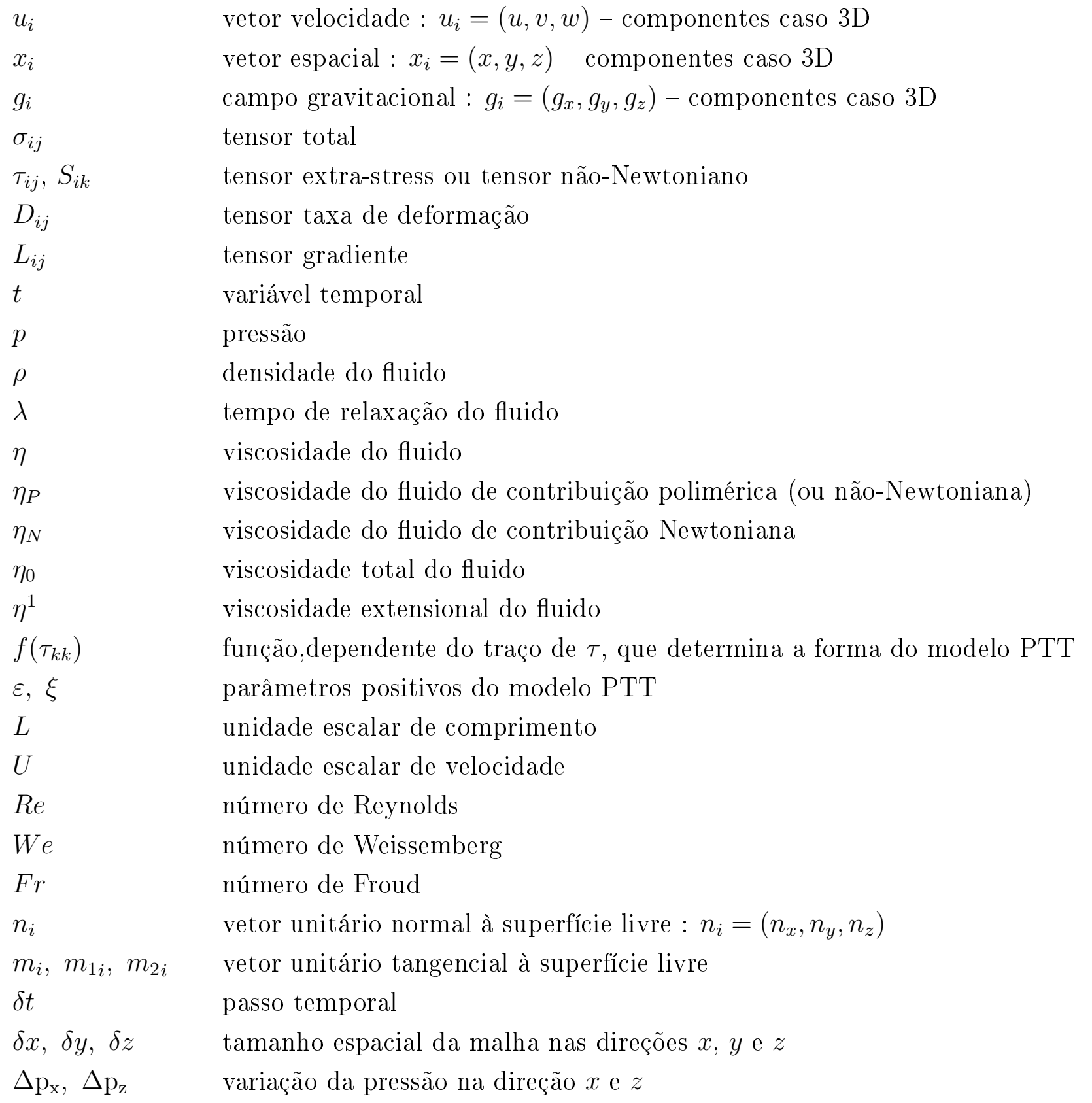


$\mathrm{E}$ (SolNum) erro relativo da solução numérica na norma $\mathbf{L}_{\mathbf{2}}$

$H$

$D$

altura do injetor até a superfície rigída

diâmetro do injetor

\section{Subescritos}

$m, n$ direção tangencial e normal, respectivamente

$i, j, k \quad$ ponto na malha, localização nas direções $x, y$ e $z$

$\max$ máximo

\section{Superescritos}

solução intermediária auxiliar

$n+1 \quad$ nível temporal

* $\quad$ nível temporal entre $n$ e $n+1$

\section{Símbolos}

$\square \quad$ derivada convectiva

$\nabla \quad$ operador gradiente

$\nabla \cdot \quad$ operador divergente

$\nabla^{2} \quad$ operador laplaciano 


\section{Lista de Figuras}

1 (a) Jato experimental, (b) Simulação do "jet buckling" com fluido Newtoniano obtido pelo código GENSMAC. . . . . . . . . . . . . . . . . . . .

1.1 Definição do domínio de dependência das variáveis para o escoamento totalmente desenvolvido em um canal bidimensional. . . . . . . . . . . . . . . . . . . . . 17

1.2 Esboço do domínio computacional para simular o escoamento em um canal bidimensional. . . . . . . . . . . . . . . . . . . . . . . . . 21

1.3 Soluções numéricas e solucões analíticas de $u, S^{x y}, S^{x x}$ e $S^{y y}$ em três malhas diferentes no tempo t $=100 \mathrm{~s}$ para $x=5$.

1.4 Simulação numérica do jato oscilante. Visualização do escoamento em diferentes tempos. Jato Newtoniano (coluna a esquerda) e jato PTT (coluna a direita) -

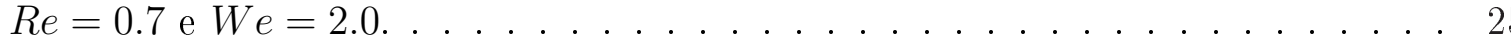

1.5 Cálculo da viscosidade extensional: o ponto em destaque representa a célula $(i, j)=(51,77)$ do domínio computacional. Esta célula está localizada no eixo de simetria do jato. . . . . . . . . . . . . . . . . . . 26

1.6 Simulação do jato oscilante: valores da viscosidade $\eta^{1}$ no ponto $(i, j)=(51,77)$ em relação ao tempo t. . . . . . . . . . . . . . . . . . . .

1.7 Simulação numérica do enchimento de um contêiner em diferentes tempos do escoamento. $R e=0.25, W e=1.5, \xi=0.2, \varepsilon=0.01$. (a esquerda) Modelo PTT - $\beta=0.1$. (a direita) Modelo PTT - $\beta=$

1.8 Tipos de fenômenos do impacto de uma gota com fluido contra uma superfície plana rígida: (a) 'bouncing'; (b) 'spreading'; (c) 'splashing' . . . . . . . . . . . . 30

1.9 Perfil de velocidade $u$ em diferentes tempo: $t=0.027 \mathrm{~s}, t=0.035 \mathrm{~s}, t=0.042 \mathrm{~s}$, $t=0.047 \mathrm{~s}, t=0.055 \mathrm{~s}, t=0.060 \mathrm{~s}$. Coluna a) fluido Newtoniano: $R e=5$, Coluna b) fluido PTT: $W e=1$, e Coluna c) fluido PTT: $W e=3 . \ldots . . . . . . .33$

2.1 Esboço do contorno de entrada de fluido. . . . . . . . . . . . . . . . . . 39

2.2 Esboço do contorno de saída de fluido. . . . . . . . . . . . . . . . . . . . . 40

2.3 Exemplo de superfície livre do fluido e vetor normal e tangencial. . . . . . . . . . 41 
2.4 Esboço do contorno rígido paralelo ao eixo-x. . . . . . . . . . . . . . 43

2.5 Esboço do contorno rígido paralelo ao eixo-y . . . . . . . . . . . . 46

2.6 Célula computacional utilizada em GENSMAC2D-PTT. . . . . . . . . . . . . 49

2.7 Tipos de células do domínio computacional. . . . . . . . . . . . . . . . 50

2.8 Célula do "inflow"com a face direita em contato com célula do interior do domínio. 53

2.9 Célula do "outflow"com a face direita em contato com célula do interior do domínio. 54

2.10 Configurações das células do contorno rígido (B) . . . . . . . . . . . . . . . 55

2.11 Célula do contorno rígido com a face inferior em contato com uma célula do interior do domínio. . . . . . . . . . . . . . . . . . . . . 56

2.12 Célula do contorno rígido com a face direita em contato com célula do interior do domínio. . . . . . . . . . . . . . . . . . . . 57

2.13 Células de superfície com apenas uma face em contato com células vazias. . . . . 59

2.14 Célula de superfície com duas faces adjacentes em contato com células vazias. . . 60

2.15 Esboço de um corte vertical em um canal bidimensional. Domínio de dependência das variáveis do problema. . . . . . . . . . . . . . . . . . 62

2.16 Definição do domínio para a simulação do escoamento em um canal bidimensional. 64

2.17 Simulação numérica do escoamento em um canal bidimensional em $t=7 \mathrm{~s}$. Isolinhas das variáveis: (a) $u,(\mathrm{~b}) \tau^{x y}$, (c) $\tau^{x x}$ e (d) $\tau^{y y}$. Dados de entrada: $R e=$ $1, W e=1.25, \xi=0.01, \varepsilon=0.5, \Delta \mathrm{p}_{\mathrm{x}}=-1.0 \ldots \ldots \ldots \ldots 6$

2.18 Simulação numérica do escoamento em um canal bidimensional em $t=100 \mathrm{~s}$. Isolinhas das variáveis: (a) $u$, (b) $\tau^{x y}$, (c) $\tau^{x x}$ e (d) $\tau^{y y}$. Dados de entrada: $R e=1, W e=1.25, \xi=0.01, \varepsilon=0.5, \Delta \mathrm{p}_{\mathrm{x}}=-1.0 \ldots \ldots \ldots \ldots$. . . . . 67

2.19 Solução numérica e solução analítica das componentes $u, \tau^{x y}, \tau^{x x}$ e $\tau^{y y}$ no meio do canal no tempo $t=100 \mathrm{~s}$. Coluna (a) M1, Coluna (b) M2, Coluna (c) M3

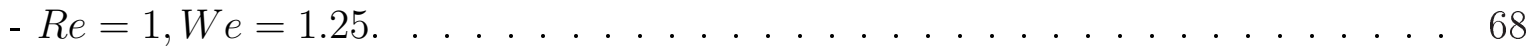

2.20 Componente $\tau^{x x}$. Solução analítica: APO. Solução numérica: $\varepsilon=0.1 ;$ e $\varepsilon=0.2$. $R e=1, W e=1.25, \xi=0.01, \Delta \mathrm{p}_{\mathrm{x}}=-1.0 \ldots \ldots \ldots \ldots$

2.21 Domínio geométrico: $5.6 \times 9.6 \mathrm{~cm} ; 56 \times 96$ células. Placa rígida de $5.6 \mathrm{~cm}$. Injetor de $7 \mathrm{~mm}$

2.22 Simulação numérica do jato oscilante em diferentes tempos. Visualização do fluido. Coluna a esquerda - Jato Newtoniano, Coluna a direita - Jato PTT: $R e=0.1, W e=0.5, \xi=0.01$ e $\varepsilon=0.01$.

2.23 Simulação numérica do jato oscilante em diferentes tempos. Visualização do fluido. Coluna a esquerda - Jato Newtoniano, Coluna a direita - Jato PTT: $R e=0.7, W e=0.5, \xi=0.01$ e $\varepsilon=0.01$.

2.24 Cálculo da viscosidade extensional: o ponto em destaque representa a célula $(i, j)=(31,80)$ do domínio computacional. Esta célula está localizada no eixo de simetria do jato. . . . . . . . . . . . . . . . . . 
2.25 Gráfico da viscosidade $\eta^{1}$ no ponto $(i, j)=(31,80)$ em função do tempo $t$. $R e=0.1, W e=0.5, \xi=0.01$ e $\varepsilon=0.01$. (a) Fluido Newtoniano. (b) Fluido viscoelástico (modelo PTT). . . . . . . . . . . . . . 76

2.26 Gráfico da viscosidade $\eta^{1}$ no ponto $(i, j)=(31,80)$ em função do tempo $t$. $R e=0.7, W e=0.5, \xi=0.01$ e $\varepsilon=0.01$. (a) Fluido Newtoniano. (b) Fluido viscoelástico (modelo PTT). . . . . . . . . . . . . . . . . . 77

2.27 Simulação numérica do jato oscilante no tempo $t=0.25 \mathrm{~s}: W e=0.5, \xi=0.01 \mathrm{e}$ $\varepsilon=0.01$. (a) $R e=0.8$ (b) $R e=0.9 \quad$ (c) $R e=1$.

2.28 Graficos da viscosidade $\eta^{1}$ no ponto $(i, j)=(31,80)$ em relação ao tempo $t$ para os jatos viscoelásticos com $R e=0.8, R e=0.9$ e $R e=1: W e=0.5, \xi=0.01 \mathrm{e}$ $\varepsilon=0.01$ fixos.

2.29 Jatos de fluidos viscoelásticos no tempo $t=0.24 \mathrm{~s}: R e=1.0, \xi=0.01$ e $\varepsilon=0.01$ nos três casos. (a) $W e=0.9$ (b) $W e=1.2 \quad$ (c) $W e=1.3 \ldots \ldots$. . . . . . .

2.30 Simulação numérica do jato oscilante nas malhas M0, M1 e M2. Dados utilizados: $R e=0.25, W e=1, \xi=0.1$ e $\varepsilon=0.1$. Configuração da superfície livre do jato em diferentes tempos: (a) $t=0.12 \mathrm{~s}$, (b) $t=0.16 \mathrm{~s}$, (c) $t=0.18 \mathrm{~s}, \quad$ (d) $t=0.21 \mathrm{~s}$.

2.31 Definição do domínio computacional para a simulação numérica do inchamento do extrudado

2.32 Simulação numérica do inchamento do extrudado. Domínio geométrico: $14.75 \mathrm{~m} \times$ $8.0 \mathrm{~m} ; 118 \times 64$ células.

2.33 Simulação do inchamento do extrudado: $R e=1$ e $W e=0.1,2.0$, 3.0. Visualização do escoamento em diferentes tempos da simulação. . . . . . . . . . . . . . 85

2.34 Razão de inchamento. $R e=1 \ldots \ldots \ldots$. . . . . . . . . 88

2.35 Simulação do inchamento do extrudado: $R e=0.1$ e $W e=0.1,1.0,2.0$. Visualização do escoamento em diferentes tempos da simulação. . . . . . . . . . . . . 89

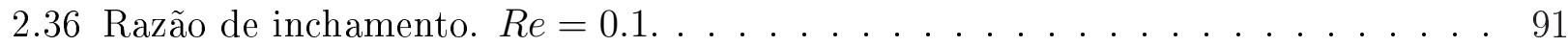

2.37 Razão de inchamento. Coluna B: $R e=1$. Coluna C: $R e=0.1 \ldots$. . . . . . . 91

2.38 Tipos de fenômenos do impacto de uma gota com fluido contra uma superfície plana rígida: (a) 'bouncing'; (b) 'spreading'; (c) 'splashing' . . . . . . . . . . . . .

2.39 Domínio geométrico: $10 \times 3.5 \mathrm{~cm} ; 100 \times 35$ células. Placa rígida de $10 \mathrm{~cm}$. Diâmetro da gota $2 \mathrm{~cm}$. Altura do centro da gota até a placa rígida $1.2 \mathrm{~cm}$. . .

2.40 Simulação do impacto de uma gota esférica com fluidoPTT contra uma placa rígida em diferentes tempos. (a) Perfil da componente $u$ de velocidade. Perfil da componente $v$ de velocidade.

2.41 Simulação do impacto de uma gota esférica de fluido Newtoniano contra uma placa rígida em diferentes tempos. (a) Perfil da componente $u$ de velocidade. (b) Perfil da componente $v$ de velocidade. 
2.42 Modelo PTT: simulação do impacto de uma gota esférica de fluido viscoelástico contra uma placa rígida em diferentes tempos. (a) Perfil da componete $\tau^{x x}$ do tensor. (b) Perfil da componente $\tau^{x y}$ do tensor. (c) Perfil da componente $\tau^{y y}$ do tensor. . . . . . . . . . . . . . . . . . . . 97

2.43 Gráficos da superfície livre da gota viscoelástica em diversos tempos da simulação. (a) $t=0.002,0.005,0.009,0.015$ s. (b) $t=0.015,0.020,0.030 \mathrm{s.}$ (c) $t=0.030,0.080 \mathrm{~s} .(\mathrm{d}) t=0.080,0.100,0.150,0.250,0.348$ s. $R e=5, W e=2$, $\xi=0.01$ e $\varepsilon=0.01$.

3.1 Esboço da superfície livre do fluido; $n_{i}$ : vetor unitário normal; $m 1_{i}, m 2_{i}$ : vetores unitários tangenciais à superfície livre do fluido. . . . . . . . . . . . . . . . . 104

3.2 Plano-xy $(\pi-x y) \ldots \ldots \ldots \ldots$. . . . . . . . . . . . . . . . . . . . . . . .

3.3 Plano-xz $(\pi-x z) \ldots \ldots \ldots \ldots \ldots \ldots \ldots$

3.4 Plano-yz $(\pi-y z) \ldots \ldots \ldots \ldots \ldots$

3.5 Célula tridimensional utilizada em GENSMAC3D-PTT. . . . . . . . . . . . . 118

3.6 Célula do contorno rígido com a face superior em contato com uma célula do interior do domínio. . . . . . . . . . . . . . . . . . . . . . . . . . . . . . 124

3.7 Tipos de superfícies planas. a) superfície-1D; b) superfície-2D; c) superfície-3D . 126

3.8 Células de superfícies (S) com apenas uma face em contato com uma face de célula vazia (E). a) face $\left(k-\frac{1}{2}\right)$ da célula $(\mathrm{S})$ em contato com uma face da célula (E); b) face $\left(k+\frac{1}{2}\right)$ da célula $(\mathrm{S})$ em contato com uma face da célula (E) . . . . 126

3.9 Células de superfícies (S) com duas faces em contato com faces de célula vazia (E).128

3.10 Células de superfícies (S) com duas faces em contato com faces de célula vazia (E).130

3.11 Domínio computacional: $7 \mathrm{~cm} \times 7 \mathrm{~cm} \times 12.8 \mathrm{~cm} ; 70 \times 70 \times 128$ células. Placa rígida de $7 \mathrm{~cm} \times 7 \mathrm{~cm} \times 3 \mathrm{~mm}$. Injetor de $6 \mathrm{~mm}$ de diâmetro. Altura do injetor até a placa rígida de $12 \mathrm{~cm}$. . . . . . . . . . . . . . . . . 132

3.12 Simulação numérica do jato oscilante. Configuração do fluido em diferentes tempos. Coluna a esquerda - jato Newtoniano; coluna a direita - jato PTT. $R e=0.5, W e=1, \xi=0.01$ e $\varepsilon=0.01 \ldots \ldots \ldots 134$

3.13 Simulação numérica do jato oscilante. Configuração do fluido em diferentes tempos. Coluna a esquerda - jato Newtoniano; coluna a direita - jato PTT.

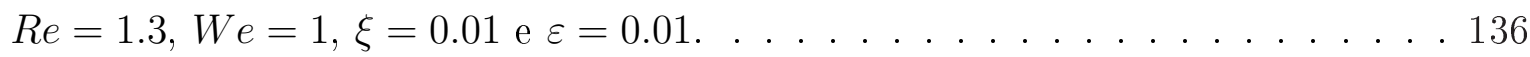

3.14 Domínio geométrico: $4.6 \times 4.6 \times 14.6 \mathrm{~m}$; Raio do injetor: $1 \mathrm{~m}$; compimento do tubo: $4 \mathrm{~m}$; Distância da extremidade do tudo até o ejetor: $10 \mathrm{~m}$. Malha utilizada: $46 \times 46 \times 146$ células $(\delta x=\delta y=\delta z=0.1 \mathrm{~m}) \ldots \ldots \ldots$. . . . . 139

3.15 Razão de inchamento obtida no tempo $t=30$ s. $R e=1$ e $W e=0.1,1$ e $3 . \quad$. . 140

3.16 Simulação do inchamento do extrudado. Visualização tridimensional do inchamento do extrudado em diferentes tempos. $R e=1$ e $W e=0.1,1,3 . \quad$. . . 141 
3.17 Simulação do inchamento do extrudado. Visualização pela face direita do inchamento do extrudado em diferentes tempos. $R e=1$ e $W e=0.1,1,3$. . . . 142 


\section{Lista de Tabelas}

1.1 Erro relativo da solução numérica, dado pela norma $L_{2}$, em cada malha considerada. 22

1.2 Modelagem do problema. Dados geométricos para as simulações de um jato incidindo contra uma placa rígida. . . . . . . . . . . . . . . . . 24

1.3 Dados que caracterizam os escoamentos dos fluidos Newtonianos e viscoelásticos. 24

1.4 Modelagem do problema. Dados geométricos para as simulações do enchimento de um contêiner. . . . . . . . . . . . . . . . . . . . 28

1.5 Dados que caracterizam os escoamentos dos fluidos Newtonianos e viscoelásticos. 28

1.6 Modelagem do problema. Dados geométricos. . . . . . . . . . . . . . . . . . . 31

1.7 Dados utilizados na simulação do impacto normal das gotas esféricas de fluido Newtoniano e viscoelástico. . . . . . . . . . . . . . . . . . . . . . 31

2.1 Erro relativo da solução numérica, dado pela norma $L_{2}$, em cada malha considerada. 69

2.2 Modelagem do problema. Geometria utilizada para o problema I e problema II . 71

2.3 Simulação numérica do jato oscilante. Dados utilizados nas simulações com jatos Newtonianos e viscoelásticos. . . . . . . . . . . . . . . . . . . 72

2.4 Dados utilizados na simulação do jato oscilante em diferentes malhas. . . . . . . 80

2.5 Simulação numérica do inchamento do extrudado. Modelagem do problema. . 83

2.6 Simulação numérica do inchamento do extrudado. Dados utilizados nas simulações com $R e=1.0 \ldots \ldots \ldots \ldots \ldots$. . . . . . . . . . 84

2.7 Simulação numérica do inchamento do extrudado. Dados utilizados nas simulações com $R e=0.1 \ldots \ldots \ldots \ldots \ldots$. . . . . . . . . 84

2.8 Simulação numérica do impacto de uma gota esférica numa placa rígida: Modelagem do problema. . . . . . . . . . . . . . . . . . . . . . . 93

2.9 Simulação numérica do impacto de uma gota esférica numa placa rígida: Dados utilizados para simular as gotas de fluido Newtoniano e viscoelástico. . . . . . . . 93

3.1 Modelagem do problema. Geometria para o Problema I e Problema II . . . . 132

3.2 Simulação numérica do jato oscilante: Dados do fluido Newtoniano e viscoelásticos. 133 
3.3 Modelagem do problema. . . . . . . . . . . . . . . . . . . . 139

3.4 Dados utilizados na simulação do inchamento do extrudado para $R e=1$. . . . 140 


\section{Sumário}

$\begin{array}{ll}\text { Introdução } & 1\end{array}$

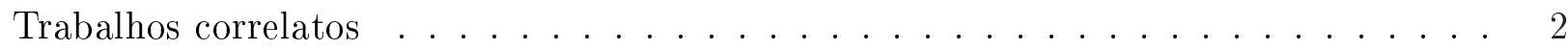

Organização da tese . . . . . . . . . . . . . . . . . 6

1 Resultados Preliminares $\quad 9$

1.1 O modelo PTT . . . . . . . . . . . . . . . . . . . . . . 9

1.2 Formulação do problema bidimensional I . . . . . . . . . . . . . . . . . 10

1.3 Adimensionalização . . . . . . . . . . . . . . . . . . . . . 11

1.4 Condições de contorno . . . . . . . . . . . . . . . . . . . . . 12

1.4.1 Contornos de entrada e saída de fluido . . . . . . . . . . . . . . . 13

1.4.2 Condições de contorno na superfície livre . . . . . . . . . . . . . . 13

1.5 Cálculo do tensor não-Newtoniano em contornos rígidos . . . . . . . . . . . . . . 14

1.5.1 Contorno rígido paralelo ao eixo- $x \ldots \ldots \ldots \ldots$. . . . . . . 14

1.5.2 Contorno rígido paralelo ao eixo-y . . . . . . . . . . . 15

1.6 Método de solução . . . . . . . . . . . . . . . . . . . . . 16

1.7 Solução analítica para escoamentos totalmente desenvolvidos em um canal bidimensional . . . . . . . . . . . . . . . . . . . . 17

1.8 Validação do método numérico . . . . . . . . . . . . . . . . . . . . . . 21

1.9 Simulação numérica de escoamentos viscoelásticos com superfícies livres . . . 23

1.9.1 Simulação numérica do jato oscilante . . . . . . . . . . . . . . . 23

1.9.2 Simulação numérica do impacto de uma gota contra uma superfície rígida 30

2 GENSMAC2D-PTT $\quad 35$

2.1 O modelo PTT . . . . . . . . . . . . . . . . . . . 35

2.2 Formulação do problema bidimensional II . . . . . . . . . . . . . . . . . . 36

2.3 Adimensionalização . . . . . . . . . . . . . . . . . . . . . 37

2.4 Condições de contorno . . . . . . . . . . . . . . . . . . . . . . 38

2.4.1 Contornos de entrada e saída de fluido . . . . . . . . . . . . . . . 39 
2.4 .2 Condições na superfície livre . . . . . . . . . . . . . . . . . . . 40

2.5 Cálculo do tensor não-Newtoniano em contornos rígidos . . . . . . . . . . . . . . 41

2.5.1 Contorno rígido paralelo ao eixo- $x \ldots \ldots \ldots \ldots$. . . . . . . . 43

2.5.2 Contorno rígido paralelo ao eixo-y . . . . . . . . . . . . . . 46

2.6 Método de solução . . . . . . . . . . . . . . . . . . . . . . . . . . . . 48

2.7 Aproximação das equações por diferenças finitas . . . . . . . . . . . . . . . . . 49

2.7.1 Classificação das Células . . . . . . . . . . . . . . . . . . . . . . . . . 49

2.7.2 Aproximação das equações básicas . . . . . . . . . . . . . . . 50

2.7.3 Aproximação das equações nos contornos . . . . . . . . . . . . . . . . 53

2.7.4 Aproximação do tensor não-Newtoniano em contornos rígidos . . . . . . 55

2.7.5 Aproximação das equações na superfície livre . . . . . . . . . . . . . . 59

2.8 Verificação do método numérico . . . . . . . . . . . . . . . . . . . 62

2.8.1 Solução analítica do modelo PTT em um escoamento totalmente desenvolvido em um canal bidimensional . . . . . . . . . . . . . . . . . 62

2.8.2 Comparação da solução numérica com a solução analítica . . . . . . . . . . 64

2.9 Simulação numérica de escoamentos viscoelásticos bidimensionais com superfícires livres . . . . . . . . . . . . . . . . . . . . . 70

2.9.1 Simulação numérica do jato oscilante . . . . . . . . . . . . . . 70

2.9.1.1 Efeito da viscoelásticidade no jato oscilante . . . . . . . . . 75

2.9.1.2 Teste de convergência: refinamento da malha . . . . . . . . . . 80

2.9.2 Simulação numérica do inchamento do extrudado . . . . . . . . . . . . . 82

2.9.3 Simulação numérica do impacto de uma gota contra uma superfície rígida 92

3 GENSMAC3D-PTT $\quad 99$

3.1 Formulação do problema tridimensional . . . . . . . . . . . . . . . . . . . . 99

3.2 Adimensionalização . . . . . . . . . . . . . . . . . . . . . . 100

3.3 Condições de contorno . . . . . . . . . . . . . . . . . . . . . . . 103

3.3 .1 Contornos de injeção e ejeção . . . . . . . . . . . . . . . . . 103

3.3.2 Condição de contorno na superfície livre . . . . . . . . . . . . . . . . . 104

3.4 Cálculo do tensor não-Newtoniano em contornos rígidos . . . . . . . . . . . . . . 105

3.4.1 Contornos rígidos paralelos ao plano-xy . . . . . . . . . . . 108

3.4.2 Contornos rígidos paralelos ao plano-xz . . . . . . . . . . 115

3.4.3 Contornos rígidos paralelos ao plano-yz . . . . . . . . . . . . . 116

3.5 Método de solução . . . . . . . . . . . . . . . . . . . . . . . . 117

3.6 Aproximação por diferenças finitas . . . . . . . . . . . . . . . 118

3.6.1 Aproximação das equações básicas . . . . . . . . . . . . . . . . . . . 119

3.6.2 Aproximação do tensor não-Newtoniano em contornos rígidos . . . . . . 123

3.6.3 Condição de contorno na superfície livre . . . . . . . . . . . . . 126

3.6.4 Cálculo do Passo no tempo . . . . . . . . . . . . . . . . . 130 
3.7 Simulação numérica de escoamentos viscoelásticos tridimensionais com superfícies livres . . . . . . . . . . . . . . . . . . . . . . . . . 131

3.7.1 Simulação numérica do jato oscilante . . . . . . . . . . . . . . . . . 131

3.7.2 Simulação numérica do inchamento do extrudado . . . . . . . . . . . . 138

4 Comentários finais e conclusões

Referências Bibliográficas 


\section{Introdução}

Há muitos séculos o homem vem pesquisando as propriedades dos fluidos em movimento. Através da compreensão dos mecanismos que governam o escoamento de fluidos vem sendo possível o desenvolvimento tecnológico em muitas áreas da ciência. Como por exemplo, o enchimento de um pote de margarina sem que o produto final contenha bolhas de ar, garantindo que o peso do produto que o consumidor está levando para casa será realmente o especificado na embalagem. Atualmemente, além desse estudo poder ser realizado combinando-se considerações teóricas sobre o escoamento de fluidos com os ensaios experimentais, esse estudo pode também ser feito através da simulação numérica. A simulação numérica só tornou-se possível a partir da década de 50 com o surgimento do computador digital e nesta época surgiu um novo ramo da ciência: Dinâmica de fluidos computacional (DFC). A dinâmica de fluidos computacional é a área da computação científica que estuda métodos computacionais para simulação de fenômenos que envolvem fluidos em movimento com ou sem trocas de calor. De forma alguma o uso desses métodos computacionais deixa de lado a prática dos estudos experimentais e das análises teóricas. Para um ensaio experimental pode-se usar dados já previstos numa simulação numérica e/ou numa análise teórica. No mesmo sentido, a análise teórica e o ensaio experimental podem validar e dar confiabilidade a uma determinada técnica numérica. E também, é através da observação nos ensaios experimentais e/ou nos resultados numéricos que despertam a curiosidade para uma nova investigação teórica. Portanto, pode-se dizer que esses três ramos: experimental, teórico e computacional se complementam.

A dinâmica de fluidos computacional é uma área muito recente, e por isso, há muitos problemas para serem investigados. Entre os diversos problemas surgidos nessa área está o tratamento da superfície livre do fluido durante o escoamento. Esse tipo de problema tem se tornado um grande desafio nessa área pois a maioria das aplicações industriais envolvem escoamentos com superfícies livres movimentando-se constantemente, tais como, extrusão e moldagem por injeção de plásticos e ligas metálicas; a injeção de fluidos utilizados na indústria alimentícia; etc. Portanto, existe um grande interesse em desenvolver métodos numéricos capazes de simular escoamentos de fluidos com superfícies livres em movimento para assim poder prever, a custos bem mais baixos e com bons resultados, o comportamento do escoamento de um fluido durante 
o processo industrial. Porém, essa não é uma tarefa fácil pois nestes problemas a superfície livre do fluido muda continuamente sua posição no tempo e no espaço tomando diferentes formas. Além disso, outra dificuldade é a aplicação das condições de contorno que devem ser satisfeitas na superfície livre cujo o formato não é conhecido e adicionalemte, muitos problemas envolvem escoamentos em geometrias altamente complexas. Diante de tantos desafios, que uma vez solucionados, podem refletir em bons resultados para a indústria. Portanto, o desenvolvimento de técnicas numéricas para simular escoamentos envolvendo superfícies livres tem sido uma área de intensa pesquisa nas últimas décadas.

\section{Trabalhos correlatos}

Uma das primeiras técnicas desenvolvidas para a simulação de escoamentos de fluidos com superfícies livres foi o método MAC (Marker-and-Cell) [33], [89] que foi introduzido por Harlow \& Welch, em Los Alamos no começo da década de 60. MAC é uma técnica de diferenças finitas que emprega variáveis primitivas velocidade e pressão. Uma das características do método MAC é o uso de partículas marcadoras que descrevem o fluido o que permite a visualização do escoamento. A partir do método MAC outros métodos numéricos foram desenvolvidos, entre eles está o método SMAC (Simplified-Marker-and-Cell) [3] cuja característica principal é a divisão do ciclo computacional em duas partes: velocidade e pressão, ou seja, não existe um processo iterativo envolvendo velocidade e pressão, fazendo assim com que algumas dificuldades do método MAC original sejam evitadas. Nos últimos anos, os método MAC e SMAC tem sido investigados por muitos pesquisadores e existem muitos trabalhos publicados baseados nos métodos MAC e SMAC e os livros de Shyy [71] e Griebel [30] abordam de forma ampla as extensões destas técnicas numéricas. Uma extensão destas técnicas numéricas que abordaremos nesse trabalho é o método GENSMAC (Generalized-Simplified-Marker-and-Cell) desenvolvido por Tomé \& McKee [85]. GENSMAC está voltado para o cálculo de escoamentos transientes bidimensionais com superfícies livres em um domínio arbitrário com condições de contorno do tipo não-escorregamento e escorregamento onde vários injetores e ejetores podem ser especificados e, assim como no método SMAC, este código utiliza partículas marcadoras para descrever o fluido, as quais fornecem a localização e a visualização da superfície livre do fluido. A princípio, Tomé \& McKee desenvolveram o código GENSMAC para simular escoamentos Newtonianos bidimensionais envolvendo superfícies livres. GENSMAC foi aplicado para simular o enchimento de contêineres e com o objetivo de validar GENSMAC, um estudo experimental desses problemas foi realizado pela empresa Unilever no fim dos anos 90. A Figura 1(a) mostra um resultado experimental de um jato Newtoniano que apresenta o fenômeno conhecido como "jet buckling" e a Figura 1(b) mostra um resultado numérico gerado pelo código GENSMAC utilizando os dados do experimento realizado. Um estudo detalhado da comparação entre o jato experimental e o resultado numérico obtido por GENSMAC pode ser encontrado no trabalho de Tomé et al., 1999 (ver [87]). Mais tarde, o código GENSMAC foi expandido para o tratamento de escoamentos de fluidos Newtonianos Generalizados na forma bidimensional [85] e Grossi et 


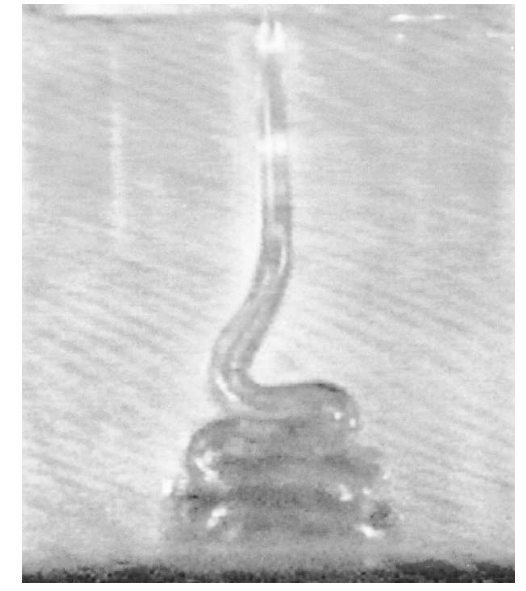

(a)

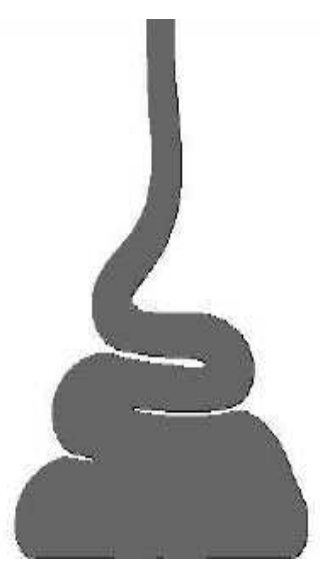

(b)

Figura 1: (a) Jato experimental, (b) Simulação do "jet buckling" com fluido Newtoniano obtido pelo código GENSMAC.

al. [31] desenvolveram uma versão do código para tratamento de escoamentos axissimétricos de fluidos Newtonianos Generalizados onde o modelo Cross é utilizado para modelar a viscosidade. A partir de 2001, GENSMAC passou a simular escoamentos bidimensionais turbulentos através da contribuição de Ferreira [28].

Motivados em obter uma técnica numérica para simular escoamentos incompressíveis tridimensionais de fluidos Newtonianos isotérmicos, Tomé et al. [80] desenvolveram o método GENSMAC3D. GENSMAC3D foi desenvolvido para simular escoamentos com superfícies livres em três dimensões e resolve as equações governantes pelo método de diferenças finitas numa malha deslocada tridimensional. O método GENSMAC3D foi implementado computacionalmente em um ambiente de simulação denominado Freeflow3D (ver [16]). O ambiente Freeflow3D é um software construído especificamente para simular escoamentos Newtonianos incompressíveis com superfícies livres em 3 dimensões. Esse ambiente é constituído de 3 módulos independentes: Modflow3D, Simflow3D e Visflow3D. O módulo Modflow3D é responsável pela modelagem de objetos geométricos tais como containers e injetores, especificação do domínio, atribuição das constantes adimensionais e inicialização de variáveis. O módulo Simflow3D é a parte central do ambiente Freeflow3D. Esse módulo implementa a discretização das equações governantes para um fluido Newtoniano incompressível utilizando a técnica GENSMAC3D [80]. O módulo Visflow3D disponibiliza a visualização dos dados gerados pelo módulo Simflow3D utilizando ferramentas de computação gráfica. Este sistema foi estruturado para permitir futuras extensões nos três módulos. Em particular, tem-se em funcionamento o movimento de contêineres [57], escoamento de fluidos não-isotérmicos [69], escoamentos multifásicos e inclusão de efeitos de tensão superficial [76] e escoamentos de fluidos Newtonianos generalizados [79].

$\mathrm{Na}$ prática muitos problemas podem ser modelados como escoamentos bidimensionais e, 
portanto, uma versão bidimensional denominada Freeflow2D foi desenvolvida por Oliveira [52]. O ambiente Freeflow2D é uma versão obtida de Freeflow3D pela supressão de uma coordenada cartesiana; assim como em Freeflow3D, Freeflow2D também possui 3 módulos distintos: Modflow2D, Simflow2D e Visflow2D. Inicialmente, Freeflow2D foi desenvolvido para simular escoamentos bidimensionais incompressíveis, isotérmicos e Newtonianos com superfícies livres (ver [52]). Com o objetivo de expandir a aplicabilidade do sistema Freeflow2D, foram introduzidos a simulação de escoamentos multifásicos [70], escoamentos axissimétricos [53], escoamentos não-isotérmicos [73], inclusão de fluidos Newtonianos Generalizados [75], escoamentos turbulentos [19]. Recentemente, Oishi [51] desenvolveu um método implícito para resolver as equações governantes para escoamentos Newtonianos, o que até então era realizado utilizando métodos explícitos.

Essencialmente tratamos aqui de escoamentos de fluidos Newtonianos os quais possuem uma viscosidade constante durante todo o escoamento, salvo a excessão dos escoamentos de fluidos Newtonianos Generalizados onde a viscosidade pode ser modelada, por exemplo, pelos modelos de Cross, Ellis, Power Law ou Carreau-Yasuda, os quais permitem calcular a viscosidade como função da taxa de cizalhamento. Assim, a simulação de escoamentos de fluidos Newtonianos Generalizados foi o primeiro passo para tratar escoamentos não-Newtonianos, que com certeza são predominantes na natureza. Há décadas, as indústrias vem desenvolvendo produtos a base de material polimérico, fluido viscoelástico - surgindo a famosa frase que sempre se ouve: "Hoje em dia, tudo é feito de plástico"; isso se dá através de processos tecnológicos como: extrusão de polímeros, injeção de moldes, enchimento de contêineres, entre outros. Desta forma, o interesse em estudar escoamentos de fluidos viscoelásticos vem crescendo em todas as áreas que abrangem esse tópico, tais como, estudos teóricos na tentativa de obter uma equação matemática capaz de descrever o escoamento de um fluido viscoelástico muito próximo do real - as equações constitutivas; estudos experimentais submetendo um determinado fluido viscoelástico a certas condições para estudar o seu comportamento; e estudos de simulação numérica que podem prever, a custos bem mais baixos dos ensaios experimentais, como o fluido viscoelástico irá se comportar. Assim, estudos para desenvolver métodos numéricos que simulem escoamentos viscoelásticos vem tomando proporção cada vez maior, não só motivados pela aplicação industrial mas também pelo fato de vencer as dificuldades inerentes das equações constitutivas, justamente por serem equações matemáticas complexas e de díficil tratamento em contornos do domínio computacional.

$\mathrm{Na}$ literatura pode-se encontrar uma vasta quantidade de artigos que tratam dos modelos viscoelásticos diferenciais de Maxwell (e.g. [6], [50]), dos modelos Oldroyd-B ([50], [63], [11], [62]), PTT (Phan-Thien-Tanner) ([60], [63], [2], [54], [92], [93]) e também de modelos integrais como por exemplo o modelo K-BKZ ([45], [44], [56], [55],[35]). O modelo de Maxwell foi uma das primeiras tentativas de descrever o efeito da viscoelasticidade de um determinado fluido. Esse modelo incorpora a idéia de um fluido que apresenta características tanto de um sólido elástico Hookeano, como de um fluido viscoso Newtoniano. Entre as diversas técnicas numéricas 
utilizadas para resolver esses modelos viscoelásticos podemos citar o trabalho [95] que utilizou o método de diferenças finitas; os trabalhos de [47], [13], [11] utilizando elementos finitos; [34], [50], [61] com volumes finitos; [6] empregando métodos espectrais; e [27] com métodos integrais de fronteira ('boundary integral methods'). Um fato que tem atraído a atenção dos pesquisadores nos escoamentos de fluidos viscoelásticos é o problema para altos números de Weissenberg. Entre os pesquisadores que estão realizando trabalhos sobre esses problemas podemos citar o Prof. Crochet e seu grupo de colaboradores em Louvain, Amstrong e Brown do MIT e os professores Tanner e Phan-Thien em Sidney. Apesar dos esforços nesta área, pouco ainda se tem feito em três dimensões mas não se pode deixar de citar os trabalhos significativos [91], [92] e [93] e, também o trabalho [43] que foi um dos principais trabalhos que apresentou um estudo numérico do fenômeno clássico conhecido como 'rod-climbing'. Este trabalho é realizado em duas dimensões (escoamentos axissimétricos).

Em três dimensões, devido a complexidade dos escoamentos de fluidos viscoelásticos, somente problemas em que a superfície livre não sofre grandes deformações ou problemas confinados tem sido tratados, como por exemplo, a simulação numérica do problema da contração que tem sido estudado em 2 e 3 dimensões ([40], [50], [63], [92], [95], [61]) e a simulação numérica do escoamento totalmente desenvolvido em um canal (2D) ou tubo (3D) ([91], [54], [2]). Escoamentos viscoelásticos envolvendo superfícies livres em movimento são importantes, mas poucos resultados têm sido publicados na literatura. Os primeiros trabalhos nesse sentido foram apresentados por Tanner [77] e Ryan \& Dutta [68]. Nestes trabalhos, eles simulam o inchamento do extrudado para o modelo Maxwell. Crochet [21] apresenta uma metodologia para resolver o problema do escoamento de um fluido Oldroyd-B para o caso do inchamento do extrudado circular e extrudado planar. Keunings, Bousfield, e co-autores ([38], [10], [39], [9]) escreveram vários artigos no final da década de oitenta (1986 e 1987) os quais tratam de escoamentos viscoelásticos com superfícies livres. Kolte et al. [42] apresentaram um estudo e os resultados da simulação 'transient filament stretching rheometer', e Yao \& McKinley [94] tratam a deformação extensional transiente em 'filament stretching devices'. Cormenzana et al. [18] estenderam o código CONNFESSITT para escoamentos com superfícies livres e apresentaram uma comparação dos seus resultados numéricos com os resultados obtidos pelo código POLYFLOW [20].

Atualmente, no laboratório LCAD/ICMC, têm-se implementado os códigos GENSMAC , Freeflow2D e Freeflow3D. O grupo de pesquisa em Matemática Computacional do LCAD tem procurado expandir esses códigos para o tratamento de escoamentos mais gerais. Grande parte dessas extensões tem sido temas de dissertações de mestrado e teses de doutorado orientadas pelos pesquisadores desse grupo. Com relação as extensões para escoamentos viscoelásticos há vários trabalhos realizados, como por exemplo, Tomé et al. [84] que apresentaram o método GENSMACVISCO para simular escoamentos viscoelásticos com superfícies livres modelados pela equação constitutiva Oldroyd-B. GENSMACVISCO foi a primeira técnica numérica a simular escoamentos viscoelásticos bidimensionais com superfícies livres dentro do código GENS- 
MAC [85], ou seja, GENSMACVISCO foi a primeira extensão do código GENSMAC para escoamentos viscoelásticos bidimensionais envolvendo superfícies livres. Outra contribuição para o código GENSMAC foi o trabalho de doutorado de Luciane Grossi [32]. Neste trabalho, o código GENSMAC é extendido para escoamentos viscoelásticos axissimétricos com superfícies livres modelados pela equação constitutiva Oldroyd-B. Também, foram temas de dissertações de mestrado as seguintes extensões no sistema Freeflow2D: escoamentos viscoelásticos bidimensionais e também axissimétricos, ambos envolvendo superfícies livres e modelados pela equação constitutiva Oldroyd-B ([72], [74]), e os escoamentos viscoelásticos bidimensionais com superfícies livres modelados pela equação constitutiva 'Upper Convected Maxwell (UCM)' [14] e fluido de segunda ordem [83]. Recentemente, Araújo [4] desenvolveu uma técnica de diferenças finitas para simular escoamentos viscoelásticos bidimensionais com superfícies livres usando a equação integral não-linear K-BKZ através do sistema Freeflow2D. A mais recente extensão é a inclusão do modelo não-linear PTT neste sistema, cuja metodologia e resultados obtidos serão apresentados em detalhes neste trabalho. Quanto as extensões no sistema Freeflow3D para escoamentos de fluidos não-Newtonianos temos como marco o trabalho de Grossi [32] (ver também Tomé et al. [79]) que desenvolveu uma técnica numérica para simular escoamentos Newtonianos Generalizados tridimensionais envolvendo superfícies livres e utiliza o modelo de Cross para calcular a viscosidade. Recentemente, Tomé et al. [82] apresentaram um trabalho que trata de escoamentos viscoelásticos tridimensionais modelados pela equação constitutiva Oldroyd-B, sendo esta mais uma extensão do sistema Freeflow3D. Neste trabalho, será mostrado o desenvolvimento de uma técnica numérica para resolver escoamentos viscoelásticos tridimensionais com superfícies livres em movimento usando a equação constitutiva não-linear PTT, tornando assim a aplicabilidade do sistema Freeflow3D cada vez mais ampla.

Portanto, o objetivo desse trabalho é expandir a aplicabilidade da simulação numérica de escoamentos viscoelásticos com superfícies livres. A equação constitutiva não-linear PTT (Phan-Thien-Tanner) ([60] e [58]) tem sido considerada um modelo realístico para polímeros fundidos e concentrados. A dificuldade em produzir um método numérico estável e robusto para resolver escoamentos viscoelásticos envolvendo superfícies livres existe até mesmo usando os modelos lineares. Portanto, é importante desenvolver um método numérico capaz de simular escoamentos viscoelásticos com superfícies livres usando o modelo PTT.

\section{Organização da tese}

Esta tese está organizada como segue.

- Capítulo 1: Apresenta as equações governantes para escoamentos viscoelásticos modelados pela equação constitutiva PTT. Descreve a primeira formulação matemática para o desenvolvimento de um método numérico capaz de simular escoamentos viscoelásticos bidimensionais envolvendo superfícies livres. Apresenta as equações envolvidas na forma adimensional, as condições de contorno e os cálculos do tensor extra-tensão nos contornos. Descreve passo-a-passo o método numérico desenvolvido e também a forma de como obter 
a solução analítica para escoamentos totalmente desenvolvidos em um canal bidimensional usando as equações resultantes da formulação matemática. Apresenta os resultados de validação bem como os resultados numéricos dos seguintes problemas: simulção numérica do jato oscilante e do impacto de uma gota contra uma superfície rígida plana. Este capítulo não apresenta a discretização das equações por meio da técnica de diferenças finitas. Isto será apresentado no Capítulo 2.

- Capítulo 2: Esse capítulo, descreve a segunda formulação matemática para o desenvolvimento de um método numérico para resolver escoamentos viscoelásticos bidimensionais envolvendo superfícies livres. Esta formulação é mais adequada porque envolve apenas os parâmetros constantes existentes no modelo PTT e não introduz parâmetros adicionais. Este capítulo apresenta as equações na forma adimensional, as condições de contorno e os cálculos do tensor extra-tensão nos contornos. Descreve o método numérico desenvolvido e também a aproximação das equações numa malha diferenciada aplicando a técnica de diferenças finitas. Apresenta os resultados de validação bem como os resultados numéricos dos seguintes problemas: simulação numérica do jato oscilante, simulação do inchamento do extrudado e simulação do impacto de uma gota contra uma superfície rígida plana.

- Capítulo 3: Esse capítulo descreve uma formulação matemática para o desenvolvimento de um método numérico para simular escoamentos viscoelásticos tridimensionais com superfícies livres em movimento. Apresenta as equações na forma adimensional, as condições de contorno e os cálculos do tensor extra-tensão nos contornos. Descreve o método numérico desenvolvido e a aproximação das equações seguindo as idéias da técnica Marker-And-Cell discretizando as equações pela técnica de diferenças finitas numa malha diferenciada (como em [81]). Por último, este capítulo apresenta os resultados numéricos dos seguintes problemas: simuação numérica do jato oscilante e do inchamento do extrudado.

- Capítulo 4: Constitui-se da conclusão ressaltando as dificuldades e os êxitos encontrados durante o trabalho. Também apresenta sugestões para trabalhos futuros. 


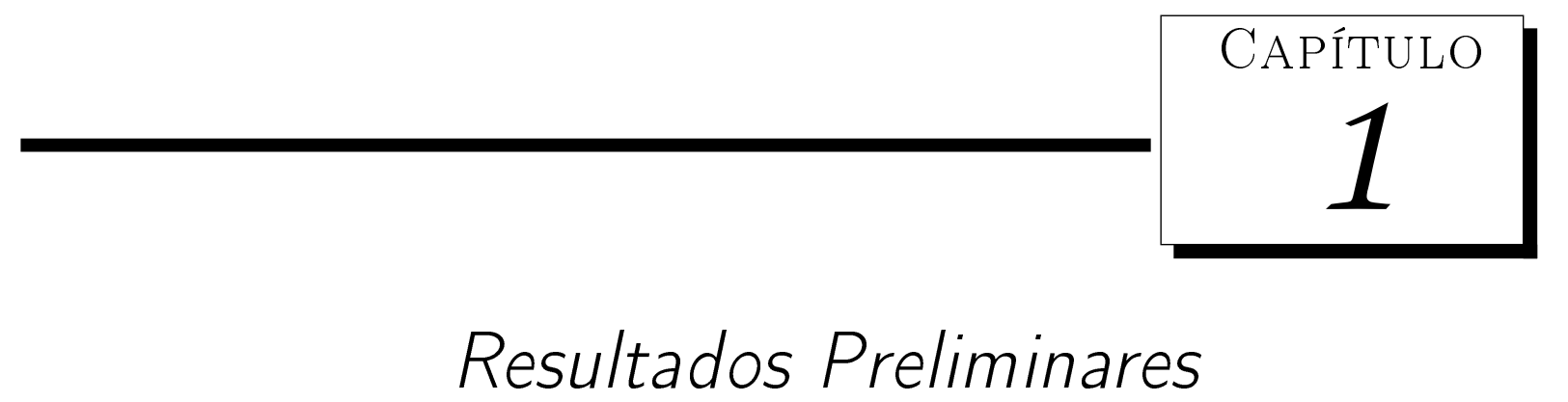

Este capítulo tem por objetivo apresentar a formulação matemática adotada inicialmente nesse projeto para a obtenção de um método numérico capaz de simular escoamentos viscoelásticos bidimensionais com superfícies livres usando a equação constitutiva PTT (Phan-Thien-Tanner). Essa formulação matemática é baseada na formulação apresentada por Xue et al. ([92] e [93]) e consiste em introduzir um novo parâmetro ao problema a ser resolvido. Este capítulo descreve as equações básicas que governam escoamentos bidimensionais incompressíveis e isotérmicos descritos pelo modelo PTT. Em seguida, é abordado uma formulação matemática do problema para obtenção do método numérico bidimensional. Para a adimensionalização das equações serão adotados os seguintes números adimensionais: número de Reynolds $(R e)$, número de Weissenberg $(W e)$ e o número de Froude $(F r)$. Os cálculos das condições de contorno e o cálculo do tensor extra-tensão em contornos rígidos não serão apresentados visto que o tratamento dessas equações será apresentado em detalhes no Capítulo 2.

\subsection{O modelo PTT}

As equações básicas que governam escoamentos incompressíveis, isotérmicos descritos pelo modelo PTT são: a equação da continuidade (conservação de massa), equação da conservação de quantidade de movimento e a equação constitutiva PTT que em notação tensorial são dadas por

$$
\begin{gathered}
\frac{\partial u_{i}}{\partial x_{i}}=0 \\
\rho \frac{\partial u_{i}}{\partial t}+\rho \frac{\partial u_{k} u_{i}}{\partial x_{k}}=-\frac{\partial p}{\partial x_{i}}+\frac{\partial \tau_{i k}}{\partial x_{k}}+\rho g_{i} \\
f\left(\tau_{k k}\right) \tau_{i j}+\lambda \stackrel{\square}{\tau}_{i j}=2 \eta_{P} D_{i j} .
\end{gathered}
$$


onde $t$ é o tempo, $x_{i}$ é o vetor espacial, $u_{i}$ é o vetor velocidade, $p$ é a pressão, $\rho$ é a densidade do fluido, $g_{i}$ é o campo gravitacional, $\tau_{i k}$ é o tensor extra-stress, o qual se relaciona com as quantidades cinemáticas através da equação constitutiva (1.3),

$$
D_{i j}=\frac{1}{2}\left(\frac{\partial u_{i}}{\partial x_{j}}+\frac{\partial u_{j}}{\partial x_{i}}\right)
$$

é o tensor taxa de deformação, $\lambda$ é o tempo de relaxação do fluido, $\eta_{P}$ é um parâmetro do modelo PTT conhecido como viscosidade do fluido.

A função $f$, dependente do traço de $\tau_{i j}$, determina qual a forma do modelo PTT:

(i) forma linear: $f\left(\tau_{k k}\right)=1+\frac{\lambda \varepsilon}{\eta_{P}} \tau_{k k}$

(ii) forma quadrática: $f\left(\tau_{k k}\right)=1+\frac{\lambda \varepsilon}{\eta_{P}} \tau_{k k}+\frac{1}{2}\left(\frac{\lambda \varepsilon}{\eta_{P}} \tau_{k k}\right)^{2}$

(iii) forma exponencial: $f\left(\tau_{k k}\right)=\exp \left(\frac{\lambda \varepsilon}{\eta_{P}} \tau_{k k}\right)$

A forma linear foi a forma proposta no paper original de Phan-Thien e Tanner [60]. Neste trabalho, vamos considerar o modelo PTT na forma linear, ou seja, a função $f$ será dada por (i).

O símbolo $(\cdot)$ representa a seguinte derivada convectiva:

$$
\stackrel{\square}{\tau}_{i j}=\frac{\partial \tau_{i j}}{\partial t}+\frac{\partial\left(u_{k} \tau_{i j}\right)}{\partial x_{k}}-\left(L_{i k}-\xi D_{i k}\right) \tau_{k j}-\left(L_{j k}-\xi D_{j k}\right) \tau_{k i}
$$

onde $L_{i j}=\partial u_{i} / \partial x_{j}$ é o tensor gradiente, a diferença $L_{i j}-\xi D_{i j}$ é chamada gradiente efetivo de velocidade [58], $\varepsilon$ e $\xi$ são parâmetros positivos do modelo PTT. O modelo SPTT (Simplified Phan-Thien-Tanner) é obtido fazendo $\xi=0$ [60] e obtém-se o modelo UCM (Upper-Convected-Maxwell) fazendo $\xi=0$ e $\varepsilon=0$ [7].

\subsection{Formulação do problema bidimensional I}

Para resolver as equações (1.1)-(1.3) empregamos a transformação EVSS (Elastic-Viscous Stress-Splitting) [64] juntamente com as idéias de Xue et al. ([92] e [93]). Essa formulação consiste em separar o tensor $\tau_{i j}$ em duas componentes, uma componente chamada de contribuição Newtoniana do fluido e a outra conhecida como contribuição não-Newtoniana e também introduz um novo parámetro $\beta$ que relaciona dois tipos de viscosidades, uma relacionada com a parte Newtoniana do fluido e a outra representa a contribuição da parte não -Newtoniana do fluido. A decomposição de $\tau_{i j}$ é dada pela equação (1.4).

$$
\tau_{i j}=2 \eta_{N} D_{i j}+S i j
$$


onde $\eta_{N}$ é a viscosidade da contribuição-Newtoniana, Sij é o tensor extra-tensão não -Newtoniano associado a contribuição polimérica do fluido.

Seja $\beta$ o parámetro de razão de retardação definido por:

$$
\beta=\eta_{P} / \eta_{0}
$$

onde $\eta_{0}=\eta_{N}+\eta_{P}$ é a viscosidade total do fluido. Dessa maneira, $\eta_{P}=\beta \eta_{0}$ e $\eta_{N}=(1-\beta) \eta_{0}$.

É interessante observar que o modelo Oldroyd-B e o modelo Maxwell(UCM) podem ser obtidos como modelos particulares do modelo PTT. Se fizermos $\xi=0, \varepsilon=0$ e $\lambda_{2}=(1-\beta) \lambda$ então obtemos o modelo Oldroyd-B; além disso, se $\beta=1$ obtemos o modelo Maxwell (UCM) [7].

Substituindo a equação (1.4) em (1.2) e (1.3) obtém-se:

$$
\begin{gathered}
\rho \frac{\partial u_{i}}{\partial t}+\rho \frac{\partial u_{k} u_{i}}{\partial x_{k}}=-\frac{\partial p}{\partial x_{i}}+(1-\beta) \eta_{0} \frac{\partial}{\partial x_{k}}\left(\frac{\partial u_{i}}{\partial x_{k}}\right)+\frac{\partial S_{i k}}{\partial x_{k}}+\rho g_{i}, \\
f\left(S_{k k}\right) S_{i j}+\lambda \stackrel{\nabla}{S}_{i j}=2 \eta_{0}\left[\beta-(1-\beta) f\left(S_{k k}\right)\right] D_{i j}-2 \lambda(1-\beta) \eta_{0} \stackrel{\nabla}{D}_{i j},
\end{gathered}
$$

$\operatorname{com} f\left(S_{k k}\right)=1+\frac{\lambda \varepsilon}{\beta \eta_{0}} S_{k k}$

Portanto, resolveremos as equações (1.1), (1.5) and (1.6) para as variáveis dependentes $u_{i}$, $p$ and $S_{i j}$.

\subsection{Adimensionalização}

Considere o sistema de coordenadas cartesiano bidimensional $x_{k}=(x, y)$ e sejam $L$ e $U$ valores de referência para comprimento e velocidade. Introduzimos a seguinte adimensionalização

$$
\begin{array}{lllll}
x=L \bar{x} & , \quad u=U \bar{u}, & g_{x}=g \bar{g}_{x}, & t=\frac{L}{U} \bar{t}, & S^{x x}=\rho U^{2} \bar{S}^{x x} \\
y=L \bar{y}, & v=U \bar{v}, & g_{y}=g \bar{g}_{y}, & p=\rho U^{2} \bar{p}, & S^{x y}=\rho U^{2} \bar{S}^{x y} \\
& S^{y y}=\rho U^{2} \bar{S}^{y y}
\end{array}
$$

Introduzindo essas variáveis adimensionais nas equações (1.1), (1.5) e (1.6) obtemos as seguintes equações adimensionais em coordenadas cartesianas bidimensionais (as barras são omitidas por conveniência):

$$
\begin{gathered}
\frac{\partial u}{\partial x}+\frac{\partial v}{\partial y}=0 \\
\frac{\partial u}{\partial t}=-\frac{\partial u^{2}}{\partial x}-\frac{\partial(v u)}{\partial y}-\frac{\partial p}{\partial x}+(1-\beta) \frac{1}{R e}\left(\frac{\partial^{2} u}{\partial x^{2}}+\frac{\partial^{2} u}{\partial y^{2}}\right)+\frac{\partial S^{x x}}{\partial x}+\frac{\partial S^{x y}}{\partial y}+\frac{1}{F r^{2}} g_{x}
\end{gathered}
$$




$$
\begin{aligned}
& \frac{\partial v}{\partial t}=-\frac{\partial(u v)}{\partial x}-\frac{\partial v^{2}}{\partial y}-\frac{\partial p}{\partial y}+(1-\beta) \frac{1}{R e}\left(\frac{\partial^{2} v}{\partial x^{2}}+\frac{\partial^{2} v}{\partial y^{2}}\right)+\frac{\partial S^{x y}}{\partial x}+\frac{\partial S^{y y}}{\partial y}+\frac{1}{F r^{2}} g_{y} \\
& \frac{\partial S^{x y}}{\partial t}=-f\left(\operatorname{tr}\left(S_{k k}\right)\right) \frac{1}{W e} S^{x y}-\frac{\partial\left(u S^{x y}\right)}{\partial x}-\frac{\partial\left(v S^{x y}\right)}{\partial y}-\left[\left(\frac{\xi}{2}-1\right) \frac{\partial v}{\partial x}+\frac{\xi}{2} \frac{\partial u}{\partial y}\right] S^{x x} \\
& -\left[\left(\frac{\xi}{2}-1\right) \frac{\partial u}{\partial y}+\frac{\xi}{2} \frac{\partial v}{\partial x}\right] S^{y y}+\left[\beta-(1-\beta) f\left(\operatorname{tr}\left(S_{k k}\right)\right)\right] \frac{1}{R e W e}\left(\frac{\partial u}{\partial y}+\frac{\partial v}{\partial x}\right) \\
& -(1-\beta) \frac{1}{R e}\left[\frac{\partial}{\partial t}\left(\frac{\partial u}{\partial y}+\frac{\partial v}{\partial x}\right)+2 \frac{\partial\left(u D^{x y}\right)}{\partial x}+2 \frac{\partial\left(v D^{x y}\right)}{\partial y}\right. \\
& \left.+2\left(\frac{\partial u}{\partial y}-\frac{\partial v}{\partial x}\right) \frac{\partial u}{\partial x}\right] \\
& \frac{\partial S^{x x}}{\partial t}=-f\left(\operatorname{tr}\left(S_{k k}\right)\right) \frac{1}{W e} S^{x x}-\frac{\partial\left(u S^{x x}\right)}{\partial x}-\frac{\partial\left(v S^{x x}\right)}{\partial y}-2(\xi-1) \frac{\partial u}{\partial x} S^{x x} \\
& -\left[(\xi-2) \frac{\partial u}{\partial y}+\xi \frac{\partial v}{\partial x}\right] S^{x y}+2\left[\beta-(1-\beta) f\left(\operatorname{tr}\left(S_{k k}\right)\right)\right] \frac{1}{R e W e} \frac{\partial u}{\partial x} \\
& -2(1-\beta) \frac{1}{R e}\left[\frac{\partial}{\partial t}\left(\frac{\partial u}{\partial x}\right)+\frac{\partial\left(u D^{x x}\right)}{\partial x}+\frac{\partial\left(v D^{x x}\right)}{\partial y}+2(\xi-1)\left(\frac{\partial u}{\partial x}\right)^{2}\right. \\
& \left.+\frac{\xi}{2}\left(\frac{\partial v}{\partial x}\right)^{2}+\left(\frac{\xi}{2}-1\right)\left(\frac{\partial u}{\partial y}\right)^{2} !+(\xi-1) \frac{\partial v}{\partial x} \frac{\partial u}{\partial y}\right] \\
& \frac{\partial S^{y y}}{\partial t}=-f\left(\operatorname{tr}\left(S_{k k}\right)\right) \frac{1}{W e} S^{y y}-\frac{\partial\left(u S^{y y}\right)}{\partial x}-\frac{\partial\left(v S^{y y}\right)}{\partial y}-2(\xi-1) \frac{\partial v}{\partial y} S^{y y} \\
& -\left[(\xi-2) \frac{\partial v}{\partial x}+\xi \frac{\partial u}{\partial y}\right] S^{x y}+2\left[\beta-(1-\beta) f\left(\operatorname{tr}\left(S_{k k}\right)\right)\right] \frac{1}{R e W e} \frac{\partial v}{\partial y} \\
& -2(1-\beta) \frac{1}{R e}\left[\frac{\partial}{\partial t}\left(\frac{\partial v}{\partial y}\right)+\frac{\partial\left(u D^{y y}\right)}{\partial x}+\frac{\partial\left(v D^{y y}\right)}{\partial y}+2(\xi-1)\left(\frac{\partial v}{\partial y}\right)^{2}\right. \\
& \left.+\frac{\xi}{2}\left(\frac{\partial u}{\partial y}\right)^{2}+\left(\frac{\xi}{2}-1\right)\left(\frac{\partial v}{\partial x}\right)^{2}+(\xi-1) \frac{\partial v}{\partial x} \frac{\partial u}{\partial y}\right]
\end{aligned}
$$

onde $R e=\frac{\rho U L}{\eta_{0}}, W e=\frac{\lambda U}{L}$ e $F r=\frac{U}{\sqrt{L|g|}}$ são os números de Reynolds, Weissenberg e Froude, respectivamente.

\subsection{Condições de contorno}

Para resolver as equações (1.8)-(1.13), deve-se impor condições de contorno apropriadas para $u_{i}=(u, v)$ e efetuar o cálculo das componentes do tensor extra-tensão $S_{i j}$ nos contornos do domínio computacional. 


\subsubsection{Contornos de entrada e saída de fluido}

Nesses contornos os valores de $u, v, S^{x x}, S^{x y}$ e $S^{y y}$ são especificados como segue.

Contorno de entrada de fluido: Na entrada de fluido as componentes do vetor velocidade são especificadas por

$$
u_{n}=U \quad \text { e } \quad u_{m}=0 .
$$

Para as componentes do tensor extra-tensão $S_{i j}$ adota-se a estratégia empregada por Crochet \& Marchal [47] e Mompean \& Deville [50], ou seja,

$$
S^{x x}=S^{x y}=S^{y y}=0 .
$$

Contorno de saída de fluido: Na saída de fluido impõem-se condições de Neumann homogêneas tanto para as componentes do vetor velocidade quanto para as componentes do tensor extra-tensão, ou seja

$$
\begin{gathered}
\frac{\partial u_{n}}{\partial n}=0, \quad \frac{\partial u_{m}}{\partial n}=0, \\
\frac{\partial S^{x x}}{\partial n}=\frac{\partial S^{x y}}{\partial n}=\frac{\partial S^{y y}}{\partial n}=0 .
\end{gathered}
$$

Nas equações acima, os subscritos $n$ e $m$ denotam direções normal e tangencial ao contorno, respectivamente.

\subsubsection{Condições de contorno na superfície livre}

Considera-se escoamentos viscosos não-estacionários com superfícies livres em contato com uma atmosfera passiva (na qual pode-se assumir pressão nula). Na ausência de tensões superficiais, as componentes do tensor tensão total $\sigma_{i j}$ devem ser contínuas nas interfaces entre dois fluidos, de maneira que na superfície livre do fluido as condições de contorno são (ver [5]).

$$
n_{i} \cdot\left(\sigma_{i j} \cdot n_{j}\right)=0 \quad \text { e } \quad m_{i} \cdot\left(\sigma_{i j} \cdot n_{j}\right)=0
$$

onde $\sigma_{i j}=-p \delta_{i j}+2(1-\beta) \frac{1}{R e} D_{i j}+S_{i j}$.

Utilizando coordenadas cartesianas bidimensionais a equação (1.14) pode ser escrita na forma adimensional

$$
\begin{gathered}
p=\frac{2(1-\beta)}{R e}\left[\left(\frac{\partial u}{\partial x} n_{x}{ }^{2}+\left(\frac{\partial u}{\partial y}+\frac{\partial v}{\partial x}\right) n_{x} n_{y}+\frac{\partial v}{\partial y} n_{y}{ }^{2}\right)\right]+S^{x x} n_{x}{ }^{2}+2 S^{x y} n_{x} n_{y}+S^{y y} n_{y}{ }^{2} \\
\frac{(1-\beta)}{R e}\left[2\left(\frac{\partial u}{\partial x}-\frac{\partial v}{\partial y}\right) n_{x} n_{y}+\left(\frac{\partial u}{\partial y}+\frac{\partial v}{\partial x}\right)\left(n_{y}^{2}-n_{x}^{2}\right)\right]+\left(S^{x x}-S^{y y}\right) n_{x} n_{y}+S^{x y}\left(n_{y}^{2}-n_{x}^{2}\right)=0,
\end{gathered}
$$

onde $n_{k}=\left(n_{x}, n_{y}\right)$ e $m_{k}=\left(n_{y},-n_{x}\right)$ são vetores unitários normal e tangencial a superfície 
livre, respectivamente.

As equações (1.15) e (1.16) representam as condições de contorno apropriadas na superfície livre.

\subsection{Cálculo do tensor não-Newtoniano em contornos rígi- dos}

No contorno rigído a velocidade obedece a condição de não-escorregamento, $u_{i j}=0$. Para o cálculo do tensor extra-tensão adota-se uma mudança de variável introduzida por Tomé et al. [84]

$$
S_{i j}=e^{-\frac{1}{W e} t} \tilde{S}_{i j}
$$

Substituindo a equação (1.17) nas equações (1.11)-(1.13) pode-se determinar o tensor extra-tensão em contornos rígidos paralelos ao eixo $x$ e ao eixo $y$, como segue.

\subsubsection{Contorno rígido paralelo ao eixo- $x$}

Nesse caso, a condição de não-escorregamento para a velocidade conduz a $\frac{\partial u}{\partial x}=0$ e $\frac{\partial v}{\partial x}=0$. Conseqüentemente pela equação da continuidade obtém-se $\frac{\partial v}{\partial y}=0$.

Dessa forma, com a mudança de variável dada pela equação (1.17) e as simplificações dadas acima obtém-se equações para as componentes $\tilde{S}^{x y}, \tilde{S}^{x x}$ e $\tilde{S}^{y y}$ através das equações (1.11)-(1.13). Resolve-se as equações para as componentes $\tilde{S}^{x y}, \tilde{S}^{x x}$ e $\tilde{S}^{y y}$, integrando-as no intervalo $[t, t+\delta t]$. Aplica-se a regra dos trapézios, o teorema do valor médio e integração por partes. Desta forma, obtém-se um sistema $3 \times 3$ para as componentes $\tilde{S}^{x y}, \tilde{S}^{x x}$ e $\tilde{S}^{y y}$. Usando a mudança de variável dada pela equação (1.17) obtém-se um sistema para as componentes $S^{x y}, S^{x x}$ e $S^{y y}$. Resolve-se esse sistema $3 \times 3$ e obtém-se as seguintes equações para o cálculo das componentes do tensor extra-tensão em contornos rígidos paralelos ao eixo $x$.

$$
\begin{aligned}
& S^{x y}(x, y, t+\delta t)=e^{-\frac{1}{W e} \delta t} S^{x y}(x, y, t)-(1-\beta) \frac{\varepsilon}{\beta} \frac{\delta t}{2}\left[\frac{\partial u(x, y, t+\delta t)}{\partial y}\left(S^{x x}(x, y, t+\delta t)+S^{y y}(x, y, t+\delta t)\right)\right. \\
& \left.+e^{-\frac{1}{W e} \delta t} \frac{\partial u(x, y, t)}{\partial y}\left(S^{x x}(x, y, t)+S^{y y}(x, y, t)\right)\right]-\frac{\xi}{2} \frac{\delta t}{2}\left[\frac{\partial u(x, y, t+\delta t)}{\partial y} S^{x x}(x, y, t+\delta t)\right. \\
& \left.\quad+e^{-\frac{1}{W e} \delta t} \frac{\partial u(x, y, t)}{\partial y} S^{x x}(x, y, t)\right]-\left(\frac{\xi}{2}-1\right) \frac{\delta t}{2}\left[\frac{\partial u(x, y, t+\delta t)}{\partial y} S^{y y}(x, y, t+\delta t)\right. \\
& \left.\quad+e^{-\frac{1}{W e} \delta t} \frac{\partial u(x, y, t)}{\partial y} S^{y y}(x, y, t)\right]+(2 \beta-1) \frac{1}{R e}\left(1-e^{-\frac{1}{W e} \delta t}\right) \frac{\partial u\left(x, y, t^{*}\right)}{\partial y} \\
& \quad-(1-\beta) \frac{1}{R e}\left[\frac{\partial u(x, y, t+\delta t)}{\partial y}-\left(1-e^{-\frac{1}{W e} \delta t}\right) \frac{\partial u\left(x, y, t^{*}\right)}{\partial y}-e^{-\frac{1}{W e} \delta t} \frac{\partial u(x, y, t)}{\partial y}\right]
\end{aligned}
$$




$$
\begin{gathered}
S^{x x}(x, y, t+\delta t)=e^{-\frac{1}{W e} \delta t} S^{x x}(x, y, t)-(\xi-2) \frac{\delta t}{2}\left[\frac{\partial u(x, y, t+\delta t)}{\partial y} S^{x y}(x, y, t+\delta t)\right. \\
\left.+\quad e^{-\frac{1}{W e} \delta t} \frac{\partial u(x, y, t)}{\partial y} S^{x y}(x, y, t)\right]-2 \frac{W e}{R e}(1-\beta)\left(\frac{\xi}{2}-1\right)\left(1-e^{-\frac{1}{W e} \delta t}\right)\left(\frac{\partial u\left(x, y, t^{*}\right)}{\partial y}\right)^{2} \\
S^{y y}(x, y, t+\delta t)=e^{-\frac{1}{W e} \delta t} S^{y y}(x, y, t)-\xi \frac{\delta t}{2}\left[\frac{\partial u(x, y, t+\delta t)}{\partial y} S^{x y}(x, y, t+\delta t)\right. \\
\left.+e^{-\frac{1}{W e} \delta t} \frac{\partial u(x, y, t)}{\partial y} S^{x y}(x, y, t)\right]-(1-\beta) \frac{W e}{R e} \xi\left(1-e^{-\frac{1}{W e} \delta t}\right)\left(\frac{\partial u\left(x, y, t^{*}\right)}{\partial y}\right)^{2}
\end{gathered}
$$

onde $t^{*} \in(t, t+\delta t)$.

\subsubsection{Contorno rígido paralelo ao eixo-y}

O cálculo das componentes do tensor extra-tensão em contornos rígidos paralelos ao eixo $y$ segue as mesmas idéias utilizadas em contornos rígidos paralelos ao eixo $x$. Nesse caso, pode-se mostrar que são dadas por

$$
\begin{gathered}
S^{x y}(x, y, t+\delta t)=e^{-\frac{1}{W e} \delta t} S^{x y}(x, y, t)-(1-\beta) \frac{\varepsilon}{\beta} \frac{\delta t}{2}\left[e^{-\frac{1}{W e} \delta t} \frac{\partial v(x, y, t)}{\partial x}\left(S^{x x}(x, y, t)+S^{y y}(x, y, t)\right)\right. \\
+\frac{\partial v(x, y, t+\delta t)}{\partial x}\left(S^{x x}(x, y, t+\delta t)+S^{y y}(x, y, t+\delta t)\right]-\left(\frac{\xi}{2}-1\right) \frac{\delta t}{2}\left[e^{-\frac{1}{W e} \delta} \frac{\partial v(x, y, t)}{\partial x} S^{x x}(x, y, t)\right. \\
\left.+\frac{\partial v(x, y, t+\delta t)}{\partial x} S^{x x}(x, y, t+\delta t)\right]-\frac{\xi \delta t}{2} \frac{\delta}{2}\left[e^{-\frac{1}{W e} \delta t} \frac{\partial v(x, y, t)}{\partial x} S^{y y}(x, y, t)\right. \\
\left.+\frac{\partial v(x, y, t+\delta t)}{\partial x} S^{y y}(x, y, t+\delta t)\right]+(2 \beta-1) \frac{1}{R e W e} e^{-\frac{1}{W e} \delta t}\left(e^{\frac{1}{W e} \delta t}-1\right) \frac{\partial v\left(x, y, t^{*}\right)}{\partial x} \\
-(1-\beta) \frac{1}{R e} e^{-\frac{1}{W e} \delta t}\left[e^{\frac{1}{W e} \delta t} \frac{\partial v(x, y, t+\delta t)}{\partial x}-\left(e^{\frac{1}{W e} \delta t}-1\right) \frac{\partial v\left(x, y, t^{*}\right)}{\partial x}-\frac{\partial v(x, y, t)}{\partial x}\right] \\
S^{x x}(x, y, t+\delta t)=e^{-\frac{1}{W e} \delta t} S^{x x}(x, y, t)-\xi \frac{\delta t}{2}\left[e^{-\frac{1}{W e} \delta t} \frac{\partial v(x, y, t)}{\partial x} S^{x y}(x, y, t)\right. \\
\left.+\frac{\partial v(x, y, t+\delta t)}{\partial x} S^{x y}(x, y, t+\delta t)\right]-(1-\beta) \frac{W e}{R e} \xi e^{-\frac{1}{W e} \delta t}\left(e^{\frac{1}{W e} \delta t}-1\right)\left(\frac{\partial v\left(x, y, t^{*}\right)}{\partial x}\right)^{2} \\
S^{y y}(x, y, t+\delta t)=e^{-\frac{1}{W e} \delta t} S^{y y}(x, y, t)-(\xi-2) \frac{\delta t}{2}\left[e^{-\frac{1}{W e} \delta t} \frac{\partial v(x, y, t)}{\partial x} S^{x y}(x, y, t)\right. \\
\left.+\frac{\partial v(x, y, t+\delta t)}{\partial x} S^{x y}(x, y, t+\delta t)\right]-2(1-\beta) \frac{W e}{R e}\left(\frac{\xi}{2}-1\right) e^{-\frac{1}{W e} \delta t\left(e^{\frac{1}{W e} \delta t}-1\right)\left(\frac{\partial v\left(x, y, t^{*}\right)}{\partial x}\right)^{2},(1.22)}
\end{gathered}
$$

onde $t^{*} \in(t, t+\delta t)$. 


\subsection{Método de solução}

O método numérico empregado para resolver as equações (1.8)-(1.13) foi baseado no método GENSMACVISCO introduzido por Tomé et al. [84] como segue.

Assumimos que num dado instante de tempo $t_{n}$ o vetor velocidade $u_{i}\left(x_{j}, t_{n}\right)$ e o tensor extra-stress $S_{i j}\left(x_{j}, t_{n}\right)$ são conhecidos e são dadas as condições de contorno para a velocidade e a pressão. Para calcular o vetor velocidade, o campo de pressão e o tensor extra-stress no tempo $t_{n+1}=t_{n}+\delta t$, ou seja, $u_{i}\left(x_{j}, t_{n+1}\right), p\left(x_{j}, t_{n+1}\right)$ e $S_{i j}\left(x_{j}, t_{n+1}\right)$ procedemos como segue:

Passo 1: Seja $\tilde{p}\left(x_{j}, t_{n}\right)$ um campo de pressão arbitrário que satisfaz a condição (1.15) na superfície livre.

Passo 2: Calcular o campo de velocidade intermediário $\widetilde{u}_{i}\left(x_{j}, t_{n+1}\right)$ por meio de

$$
\frac{\partial \widetilde{u}_{i}}{\partial t}=\frac{\partial\left(u_{k} u_{i}\right)}{\partial x_{k}}-\frac{\partial \widetilde{p}}{\partial x_{i}}+(1-\beta) \frac{1}{R e} \frac{\partial}{\partial x_{k}}\left(\frac{\partial u_{i}}{\partial x_{k}}\right)+\frac{\partial S_{i k}}{\partial x_{k}}+\frac{1}{F r^{2}} g_{i},
$$

com $\widetilde{u}_{i}\left(x_{j}, t_{n}\right)=u_{i}\left(x_{j}, t_{n}\right)$ e utilizando as mesmas condições de contorno da velocidade $u_{i}\left(x_{j}, t_{n}\right)$. Pode-se mostrar que $\widetilde{u}_{i}\left(x_{j}, t_{n+1}\right)$ tem a vorticidade correta no tempo $t_{n+1}$.

Passo 3: Resolver a equação de Poisson

$$
\nabla^{2} \psi\left(x_{j}, t_{n+1}\right)=\nabla \cdot \widetilde{u}_{i}\left(x_{j}, t_{n+1}\right) .
$$

Passo 4: Calcular a velocidade final dada por

$$
u_{i}\left(x_{j}, t_{n+1}\right)=\widetilde{u}_{i}\left(x_{j}, t_{n+1}\right)-\frac{\partial \psi\left(x_{j}, t_{n+1}\right)}{\partial x_{i}}
$$

Passo 5: Calcular a pressão :

$$
p\left(x_{j}, t_{n+1}\right)=\tilde{p}\left(x_{j}, t_{n}\right)+\frac{\psi\left(x_{j}, t_{n+1}\right)}{\delta t} .
$$

Passo 6: Calcular o tensor extra-stress $S_{i j}\left(x_{j}, t_{n+1}\right)$

(a) calcule o tensor extra-stress nos contornos: entrada de fluido, saída de fluido e contornos rígidos conforme especificado na Seção 1.4 e Seção 1.5.

(b) calcule $S_{i j}\left(x_{j}, t_{n+1}\right)$ de

$$
f\left(S_{k k}\right) S_{i j}+W e \stackrel{\nabla}{S_{i j}}=\frac{2}{R e}\left[\beta-(1-\beta) f\left(S_{k k}\right)\right] D_{i j}-2(1-\beta) \frac{W e}{R e} \stackrel{\nabla}{D}_{i j}
$$

onde $f\left(S_{k k}\right)=1+\frac{\varepsilon}{\beta} R e W e S_{k k}$, ou seja, por meio das equações (1.11)-(1.13). 
Passo 7: Atualizar as posições das partículas marcadoras: o último passo no ciclo computacional é mover as partículas marcadoras para as suas novas posições . Esse passo é efetuado resolvendo a equação

$$
\frac{d x_{i}}{d t}=u_{i}
$$

para cada partícula marcadora. A superfície livre do fluido é então definida por uma lista contendo as coordenadas dessas partículas marcadoras. A visualização da superfície livre é obtida conectando, localmente, essas partículas por retas.

\subsection{Solução analítica para escoamentos totalmente de- senvolvidos em um canal bidimensional}

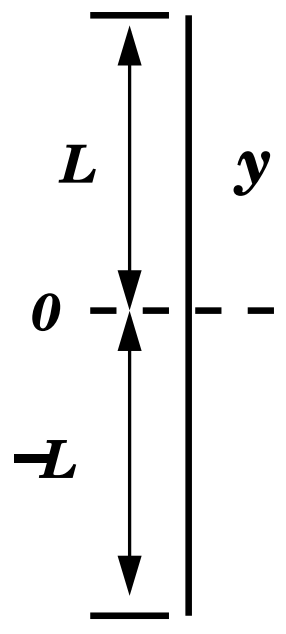

Figura 1.1: Definição do domínio de dependência das variáveis para o escoamento totalmente desenvolvido em um canal bidimensional.

Se considerarmos escoamentos totalmente desenvolvidos em um canal bidimensional temos

$$
\frac{\partial S_{i j}}{\partial t} \equiv \frac{\partial S_{i j}}{\partial x} \equiv 0, \text { onde } S_{i j} \text { é uma propriedade do fluido, } v=0, u=u(y) \text { e } \frac{\partial p}{\partial x}=\Delta \mathrm{p}_{\mathrm{x}}
$$

(constante).

Consideramos um sistema de coordenadas que é centrado no eixo de simetria $y=0$ (ver figura 1.1) e assumimos que a velocidade $u(y)$ obedece a condição de não-escorregamento em $y= \pm 1$, ou seja, $u(-1)=u(1)=0$. Além disso, impomos que a solução $u(y)$ satisfaz

$$
\frac{d u}{d y}(0)=0
$$

Neste caso, a equação de conservação de massa é automaticamente satisfeita e equação (1.9)-(1.13) reduz a

$$
\frac{\partial S^{x y}}{\partial y}=\Delta \mathrm{p}_{\mathrm{x}}-(1-\beta) \frac{1}{R e} \frac{d^{2} u}{d y^{2}}
$$




$$
\begin{gathered}
-\frac{\partial p}{\partial y}+\frac{\partial S^{y y}}{\partial y}=0 \\
-\frac{1}{W e} f\left(S_{k k}\right) S^{x x}+(2-\xi) \frac{d u}{d y} S^{x y}+(1-\beta)(2-\xi) \frac{1}{R e}\left(\frac{d u}{d y}\right)^{2}=0 \\
-\frac{1}{W e} f\left(S_{k k}\right) S^{y y}-\xi \frac{d u}{d y} S^{x y}-(1-\beta) \xi \frac{1}{R e}\left(\frac{d u}{d y}\right)^{2}=0 \\
-\frac{1}{W e} f\left(S_{k k}\right) S^{x y}-\frac{\xi}{2} \frac{d u}{d y} S^{x x}-\left(\frac{\xi}{2}-1\right) \frac{d u}{d y} S^{y y}+\left[\beta-(1-\beta) f\left(S_{k k}\right)\right] \frac{1}{R e W e} \frac{d u}{d y}=0
\end{gathered}
$$

onde $S_{k k}=S^{x x}+S^{y y}$ é o traço do tensor extra-tensão e a função $f\left(S_{k k}\right)$ é dada por

$$
f\left(S_{k k}\right)=1+\operatorname{ReWe} \frac{\varepsilon}{\beta}\left(S^{x x}+S^{y y}\right) .
$$

Para resolver o sistema de equações (1.30)-(1.35) procedemos como segue. Primeiro, integramos a equação (1.30) no intervalo $[0, y]$ para obter

$$
S^{x y}(y)-S^{x y}(0)=\Delta \mathrm{p}_{\mathrm{x}} y-(1-\beta) \frac{1}{R e}\left[\frac{d u(y)}{d y}-\frac{d u(0)}{d y}\right] .
$$

Da equação (1.29) temos $\frac{d u}{d y}(0)=0$ e neste caso pode-se ver que a solução das equação constitutiva (1.32)-(1.34) em $y=0$ é $S^{x y}(0)=S^{x x}(0)=S^{y y}(0)=0$. Assim, a equação (1.36) reduz a

$$
S^{x y}(y)=\Delta \mathrm{p}_{\mathrm{x}} y-(1-\beta) \frac{1}{R e} \frac{d u(y)}{d y} .
$$

As componentes do tensor extra-tensão $S^{x x}(y), S^{y y}(y)$ e $\frac{d u(y)}{d y}$ serão obtidas das equações (1.32)-(1.34), como segue.

Eliminando $S^{x y}$ das equações (1.32) e (1.33) provém, depois de algumas manipulações algébricas, a relação

$$
S^{y y}=-\frac{\xi}{2-\xi} S^{x x}
$$

Agora,

$$
f\left(S^{x x}+S^{y y}\right)=1+A S^{x x}
$$

onde

$$
A=\frac{2 \operatorname{Re} W e \epsilon(\xi-1)}{\beta(\xi-2)}
$$

e substituindo a equação (1.39) na equação (1.32) resulta a expressão quadrática para $S^{x x}$

$$
\frac{2 \operatorname{Re} W e \epsilon(\xi-1)}{\beta(\xi-2)}\left(S^{x x}\right)^{2}+S^{x x}-W e(2-\xi)\left[S^{x y}+\frac{(1-\beta)}{R e} \frac{d u}{d y}\right] \frac{d u}{d y}=0
$$

quando $W e \neq 0$. A equação (1.41) fornece duas soluções para $S^{x x}$. Usando o fato de que 
$\frac{d u}{d y}=0$ e $S^{x x}=0$ no meio do canal, pode-se facilmente ver que a solução procurada da equação (1.41) é dada por:

$$
S^{x x}=\frac{-1+\sqrt{1-8 R e(\epsilon / \beta)(\xi-1) \Delta \mathrm{p}_{\mathrm{x}} y \frac{d u}{d y}}}{2 A} .
$$

O parâmetro $\xi$ da equação (1.42) é em geral pequeno sendo assumido sempre $\xi<1$. Precisamos impor um gradiente de pressão negativo $\Delta \mathrm{p}_{\mathrm{x}}<0$ e a condição de não-escorregamento juntamente com a equação (1.29) implica que

$$
y \frac{d u}{d y}<0, \quad \forall y \in[-1,1]
$$

permitindo que $S^{x x}$ seja determinada da equação (1.42) para quaisquer constantes dadas $\epsilon, \beta, R e, W e$ e $0<\xi<1$.

Agora, voltamos nossa atenção para a equação (1.34). Eliminamos $S^{x x}, S^{x y}$ e $S^{y y}$ usando $(1.42),(1.37)$ e (1.38) respectivamente para obter uma equação quadrática para $\frac{d u}{d y}$. Depois de algumas manipulações algébricas, pode-se mostrar que os coeficientes de $\left(\frac{d u}{d y}\right)^{4}$ e $\left(\frac{d u}{d y}\right)^{3}$ são nulos. Assim, obtemos a seguinte equação quadrática em $\frac{d u}{d y}$ :

$$
a_{0}\left(\frac{d u}{d y}\right)^{2}+a_{1}\left(\frac{d u}{d y}\right)+a_{2}=0
$$

onde

$$
\begin{aligned}
& a_{0}=a_{0}(y)=-\operatorname{Re} W e^{2} \beta^{2} \xi(\xi-2)^{2} \Delta \mathrm{p}_{\mathrm{x}} y \\
& a_{1}=a_{1}(y)=-4 R e^{2} W e^{2} \epsilon \beta \xi(\xi-1)(\xi-2)\left(\Delta \mathrm{p}_{\mathrm{x}}\right)^{2} y^{2}-\beta^{3}[2 \epsilon(\xi-1)-\xi(\xi-2)] \\
& a_{2}=a_{2}(y)=-4 \operatorname{Re}^{3} W e^{2} \epsilon^{2}(\xi-1)^{2}\left(\Delta \mathrm{p}_{\mathrm{x}}\right)^{3} y^{3}-\beta^{2} \operatorname{Re} \Delta \mathrm{p}_{\mathrm{x}}[2 \epsilon(1-\xi)+\xi(2-\xi)] y
\end{aligned}
$$

Resolvendo a equação (1.43), obtemos

$$
\left(\frac{d u}{d y}\right)_{1}=\left(-a_{1}+\sqrt{a_{1}^{2}-4 a_{0} a_{2}}\right) / 2 a_{0}
$$

$\mathrm{Ou}$

$$
\left(\frac{d u}{d y}\right)_{2}=\left(-a_{1}-\sqrt{a_{1}^{2}-4 a_{0} a_{2}}\right) / 2 a_{0} .
$$

Das equações (1.44)-(1.46) pode-se ver que em $y=0$ temos $a_{0}(0)=a_{2}(0)=0$ enquanto $a_{1}(0)=-\beta^{3}[2 \epsilon(\xi-1)-\xi(\xi-2)] \neq 0$ em geral. Claramente (1.48) é ilimitada em $y=0$ e portanto deve ser descartada. Além do mais, pode-se mostrar que $\lim _{y \rightarrow 0}\left(\frac{d u}{d y}\right)_{1}=0$. Portanto, 
podemos afirmar que $\left(\frac{d u}{d y}\right)_{1}$ é a solução procurada. Após algumas manipulações podemos escrever

$$
\left(\frac{d u}{d y}\right)_{1}=\frac{d u}{d y}=-\frac{2 R e \Delta \mathrm{p}_{\mathrm{x}}}{\beta \chi} y+\frac{1}{2 W e^{2} \xi(2-\xi) \Delta \mathrm{p}_{\mathrm{x}}}(1+2 / \chi)\left(\frac{1-\sqrt{1-(a y)^{2}}}{y}\right)
$$

onde

$$
\chi=\frac{\xi(2-\xi)}{\epsilon(1-\xi)}
$$

e

$$
a=-2 \frac{R e}{\beta} W e \sqrt{\xi(2-\xi)} \Delta \mathrm{p}_{\mathrm{x}} .
$$

Integrando a equação (1.49) obtém-se

$$
\begin{aligned}
& u(y)=\frac{R e \Delta \mathrm{p}_{\mathrm{x}}}{\beta \chi}\left(1-y^{2}\right) \\
& +\frac{1}{2 W e^{2} \xi(2-\xi) \Delta \mathrm{p}_{\mathrm{x}}}(1+2 / \chi)\left\{\sqrt{1-a^{2}}-\sqrt{1-(a y)^{2}}+\ln \left[\left|1+\frac{\sqrt{1-(a y)^{2}}}{1+\sqrt{1-a^{2}}}\right|\right]\right\}
\end{aligned}
$$

A componente $S^{x y}$ do tensor extra-stress é calculada facilmente de (1.37), sendo

$$
S^{x y}=\left(1+\frac{2(1-\beta)}{\beta \chi}\right) \Delta \mathrm{p}_{\mathrm{x}} y-\frac{(1-\beta)}{2 \operatorname{ReW} e^{2} \xi(2-\xi) \Delta \mathrm{p}_{\mathrm{x}}}(1+2 / \chi)\left(\frac{1-\sqrt{1-(a y)^{2}}}{y}\right)
$$

Para calcular $S^{x x}$ substituimos a equação (1.52) $\left(\frac{d u}{d y}\right)$ na equação (1.42) e após algumas manipulações algébricas, obtemos

$$
\begin{aligned}
& S^{x x}=\frac{\beta(\xi-2)}{4 \operatorname{Re} W e \epsilon(\xi-1)}\{-1 \\
& \left.+\sqrt{1-8 \frac{R e}{\beta} W e^{2} \epsilon(\xi-1)\left(1+2 \frac{(1-\beta)}{\beta \chi}\right)\left(\Delta \mathrm{p}_{\mathrm{x}}\right)^{2} y^{2}+4 \frac{(1-\beta)}{\beta \chi}\left(1+\frac{2}{\chi}\right)\left(1-\sqrt{1-(a y)^{2}}\right)}\right\}
\end{aligned}
$$

e conseqüentemente $S^{y y}$ pode ser calculada utilizando a equação

$$
S^{y y}=-\left(\frac{\xi}{2-\xi}\right) S^{x x}
$$

Portanto, a solução analítica para as componentes do tensor extra-tensão $S^{x y}, S^{x x}$ e $S^{y y}$ e para a velocidade $u$ nos escoamentos cartesianos bidimensionais em estado estácionario são dadas pelas equações (1.37), (1.52), (1.54), (1.55), respectivamente. 


\subsection{Validação do método numérico}

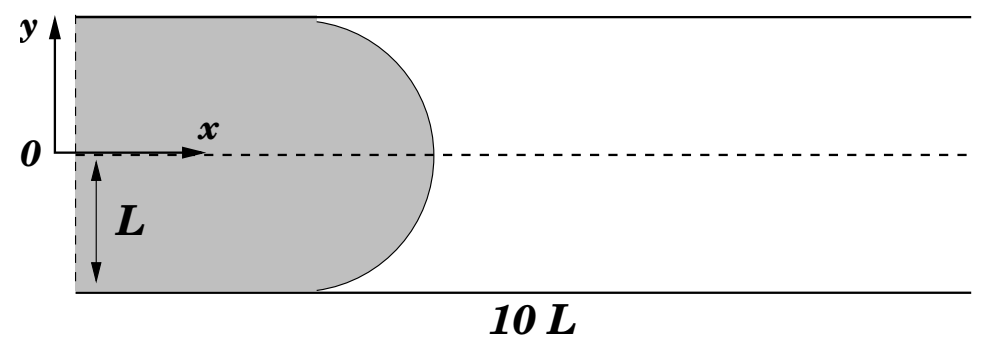

Figura 1.2: Esboço do domínio computacional para simular o escoamento em um canal bidimensional.

Para validarmos o método numérico apresentado na Seção 1.6, simulamos o escoamento em um canal bidimensional governado pelo modelo PTT e comparamos os resultados numéricos com a solução analítica obtida na Seção 1.7, como segue.

Consideramos um canal bidimensional formado por duas placas paralelas a uma distancia $L$ do eixo de simetria $y=0$ tendo o comprimento de $10 L$. Na entrada do canal impomos os perfis analíticos para as componentes do tensor extra-tensão $S^{x y}, S^{x x}$ e $S^{y y}$, e para a velocidade $u$ obtidas na Seção 1.7 (conforme as equações (1.37), (1.52), (1.54) e (1.55)).

No tempo $t=0$ o canal está complementamente vazio; o fluido é injetado na entrada do canal a uma velocidade prescrita até atingir a saída do canal; após $t=100 \mathrm{~s}$ de simulação o escoamento atinge o estado estacionário. No estado estacionário os valores da velocidade e das componentes do tensor extra-tensão são os mesmos que os impostos na entrada do canal. Para verificar este fato e comparar os resultados numéricos com a solução analítica, dada pelas equações (1.37), (1.52), (1.54) e (1.55), foram feitas três simulações utilizando malhas diferentes:

Malha $1(M 1): 80 \times 400$ celúlas,

Malha $2(M 2): 40 \times 200$ celúlas,

Malha $3(M 3): 20 \times 100$ celúlas.

Nessas simulações, os seguintes dados de entrada foram utilizados:

$L=1.0 \mathrm{~m}, \quad U=U \max =1.273341654 \mathrm{~ms}^{-1}, \quad \nu=1.273341654 \mathrm{~m}^{2} \mathrm{~s}^{-1}, \quad \lambda=0.314134073 \mathrm{~s}$, $\varepsilon=0.01, \xi=0.1, \quad \beta=0.7, \Delta \mathrm{p}_{\mathrm{x}}=-1.6, R e=1.0 \quad$ e $W e=0.4$.

Os resultados dessas simulações estão mostrados na figura 1.3. Os valores da solução numérica obtidos para a velocidade $u$ e para as componentes do tensor extra-tenão $S^{x y}, S^{x x}$ e $S^{y y}$ nas malhas $(M 1),(M 2)$ e (M3) no meio do canal estão sendo representados por símbolos na figura 1.3, enquanto as soluções analíticas estão sendo representadas por linhas contínuas.

Como pode-se observar na figura 1.3, existe uma ótima concordância entre a solução numérica e a solução exata.

Os erros relativos entre a solução analítica e a solução numérica, representadas por SolEx e 

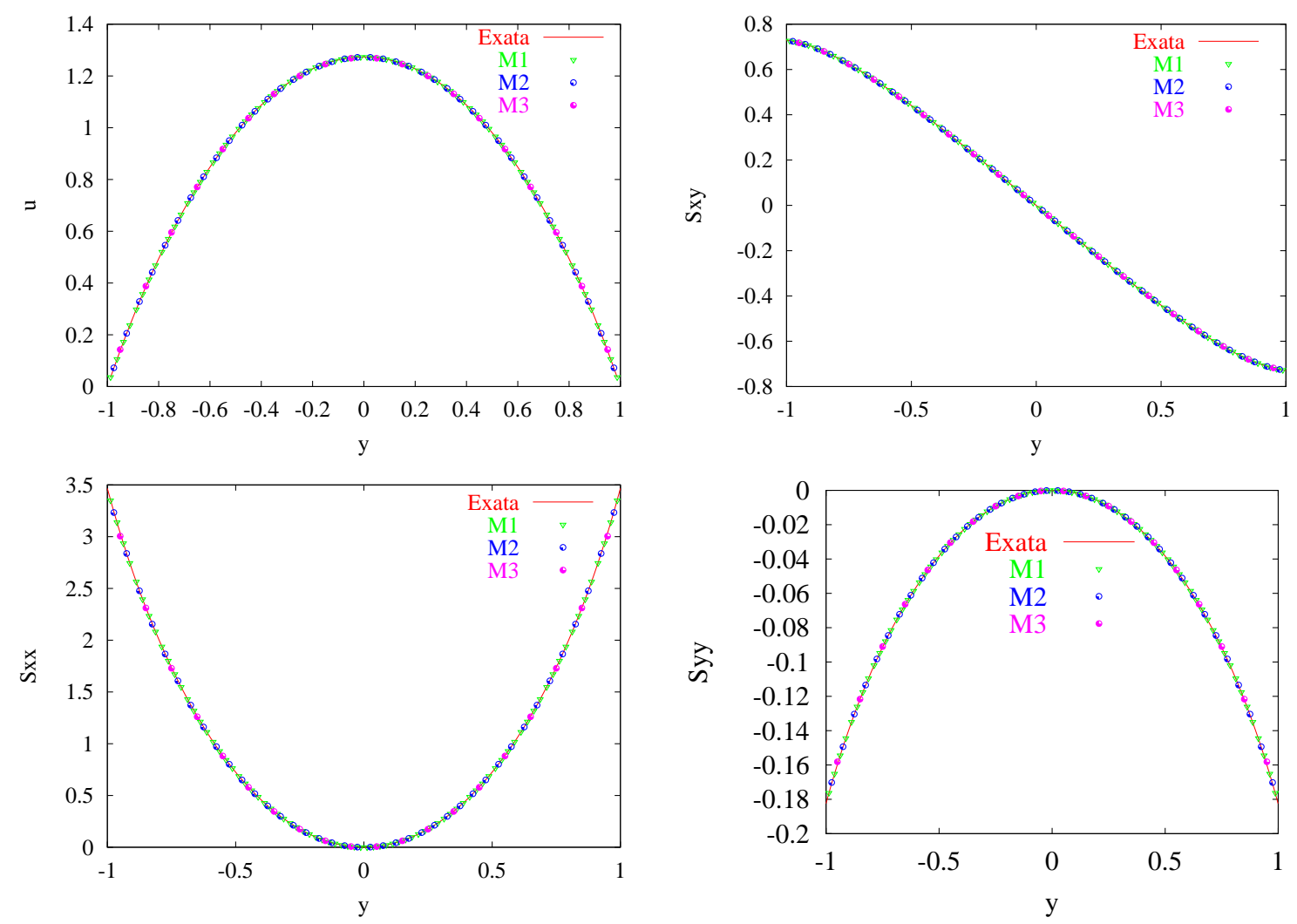

Figura 1.3: Soluções numéricas e solucões analíticas de $u, S^{x y}, S^{x x}$ e $S^{y y}$ em três malhas diferentes no tempo $\mathrm{t}=100 \mathrm{~s}$ para $x=5$.

\begin{tabular}{|c|c|c|c|c|}
\hline Malha & $E(u)$ & $E\left(\Sigma^{x y}\right)$ & $E\left(\Sigma^{x x}\right)$ & $E\left(\Sigma^{y y}\right)$ \\
\hline \hline$M 1$ & $24.4010^{-9}$ & $18.5610^{-9}$ & $16.0610^{-8}$ & $16.9610^{-8}$ \\
\hline$M 2$ & $32.2510^{-8}$ & $23.0810^{-8}$ & $29.3110^{-7}$ & $29.3010^{-7}$ \\
\hline$M 3$ & $37.2110^{-7}$ & $24.9910^{-7}$ & $48.5310^{-6}$ & $48.2610^{-6}$ \\
\hline
\end{tabular}

Tabela 1.1: Erro relativo da solução numérica, dado pela norma $L_{2}$, em cada malha considerada.

SolNum respectivamente, dados por

$$
E(\operatorname{SolNum})=\sqrt{\sum_{j}\left(\operatorname{SolEx}\left(y_{j}\right)-\operatorname{SolNum}_{M_{i}}\left(y_{j}\right)\right)^{2}} .
$$

são apresentados na Tabela 1.1 na qual pode-se observar que o erro decresce a medida em que a malha é refinada. Isso mostra que o método numérico desenvolvido nesse trabalho é convergente. 


\subsection{Simulação numérica de escoamentos viscoelásticos com superfícies livres}

Nesta seção pretende-se demonstrar que o sistema Freeflow2D pode simular escoamentos viscoelásticos com superfícies livres em movimento governados pela equação constitutiva não -linear PTT. Consideramos o problema de um jato de fluido viscoelástico que ao incidir numa placa rígida pode apresentar um fênomeno conhecido como flambagem ou jato oscilante ('jet buckling') e o problema de uma gota de fluido viscoelástico incidindo perpendicularmente contra uma superfície rígida plana - problema 'impacting drop'.

\subsubsection{Simulação numérica do jato oscilante}

No momento em que um jato a baixo número de Reynolds incide numa superfície rígida plana, pode ocorrer o fênomeno conhecido como flambagem ou jato oscilante. Esse fênomeno tem atraído um grande número de pesquisadores e tem sido estudado tanto experimentalmente quanto numericamente. Cruickshank \& Munson [23] e Cruicksanck [22] (ver também [67], [66], [8]) apresentaram resultados teóricos e experimentais para jatos Newtonianos e obtiveram uma estimativa para quando o fenômeno do jato oscilante ocorre. A estimativa encontrada mostra uma relação entre o numéro de Reynolds e a razão $H / D$ ( $H$ é a altura do injetor até a superfície rigída e $D$ é o diâmetro do injetor). Cruickshank e Munson mostraram que um jato Newtoniano bidimensional sofrerá oscilações se a condição (1.57) for satisfeita:

$$
R e<0.56 \text { e } H / D>3 \pi
$$

Para o caso do jato Newtoniano axissimétrico eles mostraram que se a condição (1.58)

$$
R e<1.2 \text { e } H / D>2 \pi \text {. }
$$

for satisfeita então o jato oscilará. Tomé \& McKee [86] investigaram este problema numéricamente realizando simulações com jatos bidimensionais. As simulações numéricas para o caso tridimensional foram feitas por Castelo et al. [15] e recentemente por Tomé et al. [79]. Nesse caso, os autores simularam o escoamento de um jato tridimensional de um fluido Newtoniano através de um injetor no formato de um paralelepípedo. Considerando fluidos viscoelásticos, a primeira investigação do grupo de pesquisa do LCAD (Laboratório Computacional de Alto Desempenho) do ICMC/USP com fluidos viscoelásticos foi feita por Tomé et al. [84] onde os autores estudaram o escoamento de um jato de fluido viscoelástico modelado pela equação constitutiva Oldroyd-B. Com o intuito de expandir a aplicabilidade do sistema Freeflow2D para escoamentos viscoelásticos vamos demonstrar nesta seção que o método numérico é capaz de tratar o problema do jato oscilante para fluidos viscoelásticos governados pelo modelo PTT.

O número de Reynolds tem um carácter importante que prediz o comportamento do escoamento de um jato Newtoniano quanto ao fenômeno do jato oscilante. Entretanto, para um 
jato com fluido viscoelástico modelado pela equação constitutiva PTT o número de Reynolds não é constante durante o escoamento pois o valor da viscosidade varia com o valor da taxa de deformação local e com o tempo. Para ilustrar que a viscoelasticidade tem uma forte influência no fenômeno oscilatório do jato apresentamos duas simulações : uma usando fluido Newtoniano e a outra empregando o modelo PTT. Os dados geométricos utilizados nessas simulações estão dispostos na Tabela 1.2 e os dados que caracterizam os fluidos, tanto os Newtonianos quanto os viscoelásticos, estão dispostos na Tabela 1.3.

\begin{tabular}{|l|l|}
\hline espaçamento da malha & $\delta x=\delta y=1 \mathrm{~mm}$ \\
\hline$H:$ altura do injetor até a placa rígida & $9 \mathrm{~cm}$ \\
\hline$D:$ diâmetro do injetor & $7 \mathrm{~mm}$ \\
\hline
\end{tabular}

Tabela 1.2: Modelagem do problema. Dados geométricos para as simulações de um jato incidindo contra uma placa rígida.

Pode-se observar na Tabela 1.2 que $H / D=12.85>3 \pi$. Dessa forma, escolhemos $R e=$ $U D / \nu=0.7$ para as simulações, ou seja, escolhemos o número de Reynolds de forma que a condição (1.57) não seja satisfeita.

\begin{tabular}{|l|l|}
\hline Newtoniano e PTT & Simulações com Re $=0.7$ \\
\hline \hline$U:$ velocidade no injetor & $0.7 \mathrm{~ms}^{-1}$ \\
\hline$g_{x}:$ força gravitacional & $-9.81 \mathrm{~ms}^{-2}$ \\
\hline$\nu=\eta_{0} / \rho$ & $0.007 \mathrm{~m}^{2} \mathrm{~s}^{-1}$ \\
\hline$R e$ & $\mathbf{0 . 7}$ \\
\hline$F r$ & 2.671250 \\
\hline modelo PTT & \\
\hline$\lambda$ & $0.02 \mathrm{~s}$ \\
\hline$\varepsilon$ & 0.5 \\
\hline$\xi$ & 0.1 \\
\hline$\beta$ & 0.9 \\
\hline$W e$ & 2.0 \\
\hline
\end{tabular}

Tabela 1.3: Dados que caracterizam os escoamentos dos fluidos Newtonianos e viscoelásticos.

Logo, temos que $H / D>3 \pi$ e $R e=0.7>0.56$ e portanto, pela teoria de Cruickshank o jato Newtoniano não apresentará o fenômeno oscilatório. Os resultados dessas simulações são mostrados na figura 1.4. De fato, como era esperado pela teoria de Cruickshank o jato Newtoniano não sofreu nenhuma oscilação ao atingir a placa rígida. Porém, o jato viscoelástico apresentou o fênomeno oscilatório, ou seja, a teoria de Cruickshank não se aplica ao jato bidimensional de fluido viscoelástico. Isso se deve ao fato de que a viscosidade do jato contendo o fluido viscoelástico não é constante durante o escoamento e varia com o valor da taxa de deformação local.

Com efeito, em escoamentos na direção y puramente extensional planar, a viscosidade do 

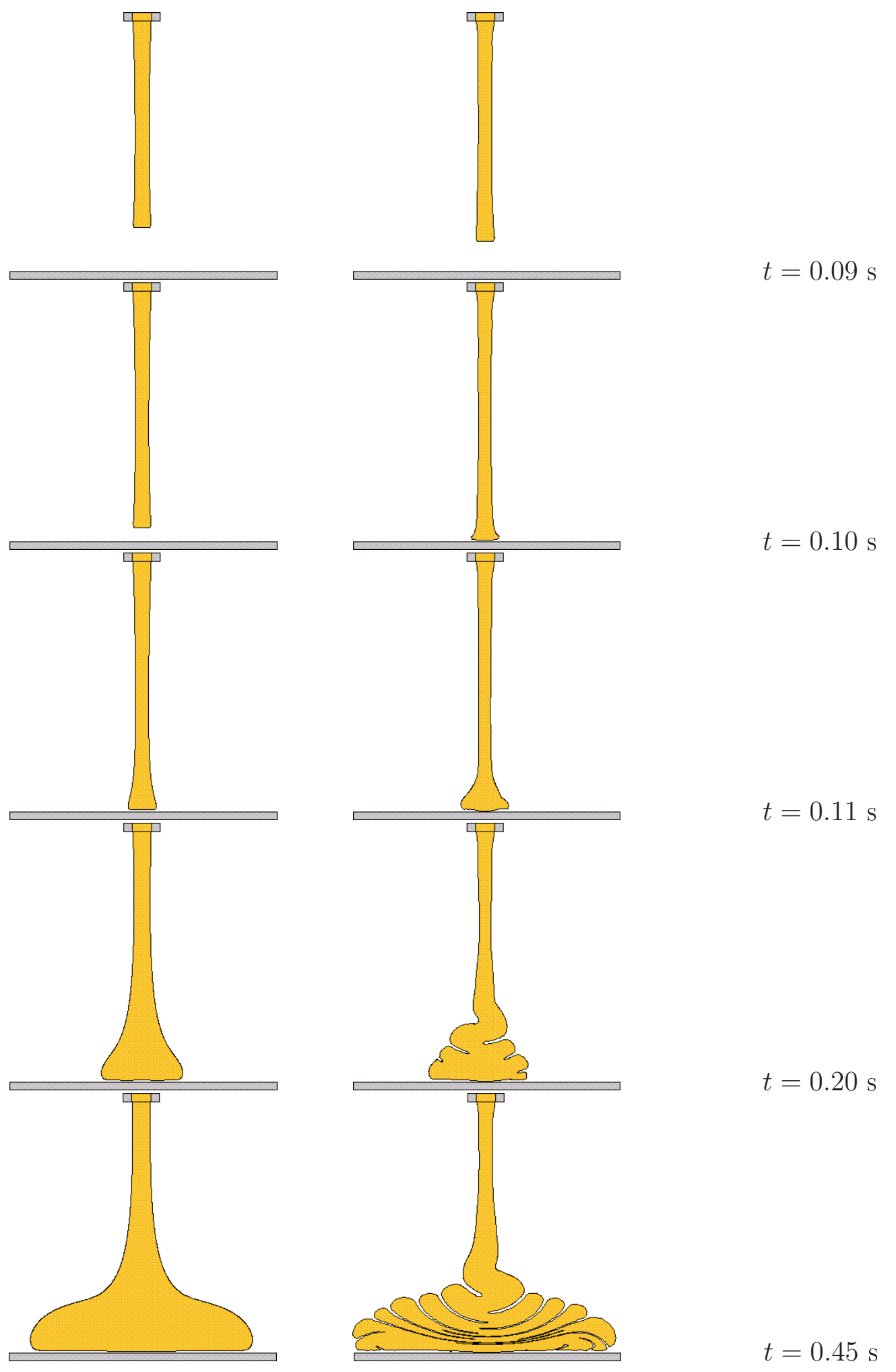

Figura 1.4: Simulação numérica do jato oscilante. Visualização do escoamento em diferentes tempos. Jato Newtoniano (coluna a esquerda) e jato PTT (coluna a direita) - Re $=0.7 \mathrm{e}$ $W e=2.0$. 
jato viscoelástico pode ser calculada pela equação (1.59) (ver [46])

$$
\eta^{1}=\frac{\tau^{y y}-\tau^{x x}}{D^{y y}}
$$

Essa viscosidade varia no decorrer do escoamento com o tempo e com o valor da taxa de deformação local. Para mostrar esse fato, consideramos um ponto fixo ao longo do eixo de simetria do jato e calculamos a viscosidade pela equação (1.59) durante a descida do jato. Escolhemos o ponto mostrado na figura 1.5 situado na célula $(i, j)=(51,77)$ e calculamos a viscosidade no intervalo de tempo $[0.09 s, 0.11 s]$. Esse intervalo de tempo é uma vizinhança do tempo em que o jato atinge a placa rígida e durante esse tempo podemos dizer que o escoamento é puramente elongacional. Após o jato atingir a placa rígida teremos então um escoamento complexo, envolvendo também cisalhamento.

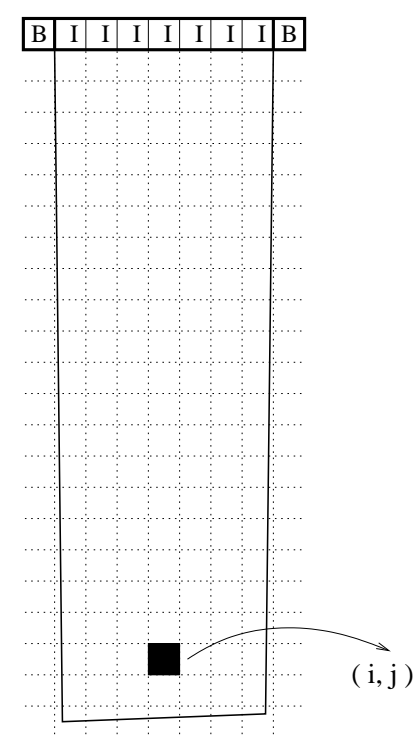

Figura 1.5: Cálculo da viscosidade extensional: o ponto em destaque representa a célula $(i, j)=$ $(51,77)$ do domínio computacional. Esta célula está localizada no eixo de simetria do jato.

Os valores da viscosidade extensional calculados em função do tempo na célula $(i, j)=$ $(51,77)$ são mostrados na figura 1.6. Podemos observar no gráfico 1.6 que a viscosidade do fluido Newtoniano é constante durante todo o intervalo de tempo considerado. Além disso, o valor da viscosidade $\eta^{1}$ para o fluido Newtoniano pode ser verificado pela definição $\tau_{i j}=\frac{2}{R e} D_{i j}$ e pela equação de conservação de massa:

$$
\eta^{1}=\frac{\tau^{y y}-\tau^{x x}}{D^{y y}}=\frac{\frac{2}{R e} \frac{\partial v}{\partial y}-\frac{2}{R e} \frac{\partial u}{\partial x}}{\frac{\partial v}{\partial y}}=\frac{4}{R e}=\frac{4}{0.7}=5.71428
$$

Por outro lado, a viscosidade do fluido viscoelástico está crescendo durante todo o intervalo de tempo considerado e toma valores superiores ao da viscosidade Newtoniana após o jato atingir a placa rígida. Isso faz com que o jato fique mais viscoso reduzindo o número de Reynolds local 


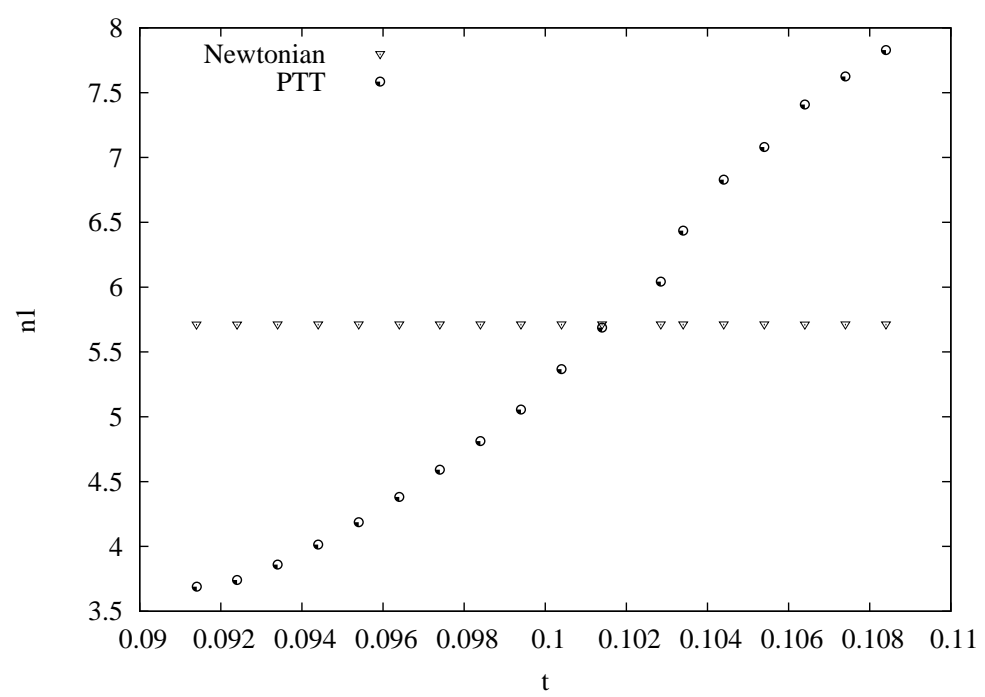

Figura 1.6: Simulação do jato oscilante: valores da viscosidade $\eta^{1}$ no ponto $(i, j)=(51,77)$ em relação ao tempo $t$.

o que torna o escoamento mais lento fazendo com que o jato oscile.

Para mostrar a influência do parâmetro $\beta$ no escoamento, realizamos duas simulações do enchimento de um contêiner com fluido viscoelástico governado pelo modelo PTT onde em cada uma dessas simulações utilizamos diferentes valores para o parâmetro $\beta$ e mantivemos fixos os demais parâmetros do problema. A Tabela 1.4 apresenta os dados geométricos utilizados em ambas as simulações. Os dados que caracterizam os fluidos viscoelásticos governados pelo modelo PTT, estão dispostos na Tabela 1.5. A figura 1.7 mostra os resultados obtidos nessas simulações nos tempos $t=0.12,0.14,0.30,0.44$ e $0.62 \mathrm{~s}$. No tempo $t=0.12 \mathrm{~s}$ as duas simulações ainda não atingiram o contêiner e aparentemente mostram o mesmo comportamento. No tempo $t=0.14 \mathrm{~s}$ ambos os fluidos atingiram o contêiner e então pode-se notar uma pequena diferença nos jatos. O jato com o parâmetro $\beta=0.1$ apresenta uma leve assimetria enquanto o jato com o parâmetro $\beta=0.9$ mantêm-se simétrico. No entanto, a partir do tempo $t=0.30 \mathrm{~s}$ podemos observar que ambos os jatos apresentam o fenómeno oscilatório e podemos notar que o jato com $\beta=0.9$ apresenta um efeito mais viscoso que o jato com $\beta=0.1$. Acreditamos que esse efeito é devido a alta viscoelasticidade do fluido porque pela definição do parâmetro $\beta$ temos: $\beta=\frac{\eta_{P}}{\eta_{N}+\eta_{P}}$ temos que $\beta \rightarrow 1$ quando $\eta_{N} \rightarrow 0$ e $\beta \rightarrow 0$ quando $\eta_{N} \rightarrow \infty$. Portanto, para $\beta=0.9$, o parâmetro viscosidade predominante é a viscosidade polimérica $\eta_{P}$ assim os efeitos viscoelásticos são mais fortes no escoamento e o jato apresenta efeitos provenientes da elasticidade do fluido. 


\begin{tabular}{|l|l|}
\hline espaçamento da malha & $\delta x=\delta y=1 \mathrm{~mm}$ \\
\hline$H:$ altura do injetor até a placa rígida & $5 \mathrm{~cm}$ \\
\hline$D:$ diâmetro do injetor & $6 \mathrm{~mm}$ \\
\hline
\end{tabular}

Tabela 1.4: Modelagem do problema. Dados geométricos para as simulações do enchimento de um contêiner.

\section{Dados que caracterizam os escoamentos dos fluidos viscoelásticos}

\begin{tabular}{|l|l|}
\hline$U:$ velocidade no injetor & $0.25 \mathrm{~ms}^{-1}$ \\
\hline$g_{x}:$ força gravitacional & $-9.81 \mathrm{~ms}^{-2}$ \\
\hline$\nu=\eta_{0} / \rho$ & $0.006 \mathrm{~m}^{2} \mathrm{~s}$ \\
\hline$R e$ & 0.25 \\
\hline$F r$ & 1.030457 \\
\hline$\lambda$ & $0.036 \mathrm{~s}$ \\
\hline$W e$ & 1.5 \\
\hline$\varepsilon$ & 0.01 \\
\hline$\xi$ & 0.2 \\
\hline$\beta$ & $\mathbf{0 . 1}$ e $\mathbf{0 . 9}$ \\
\hline
\end{tabular}

Tabela 1.5: Dados que caracterizam os escoamentos dos fluidos Newtonianos e viscoelásticos. 

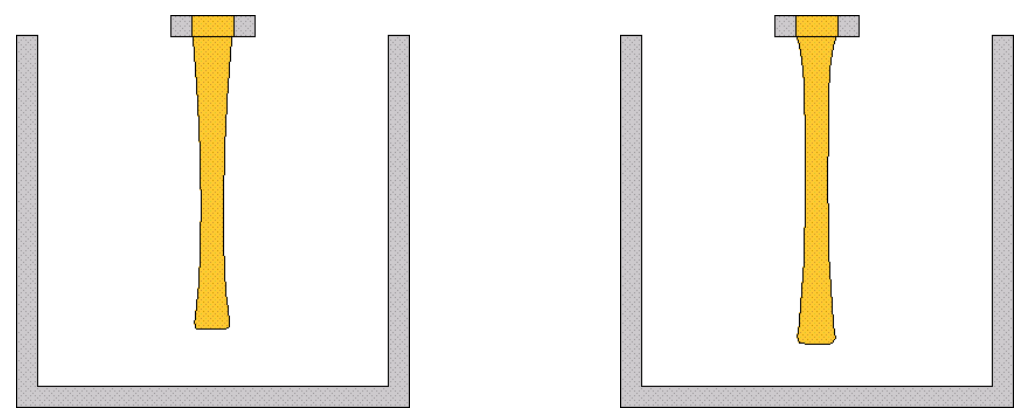

$t=0.12 \mathrm{~s}$
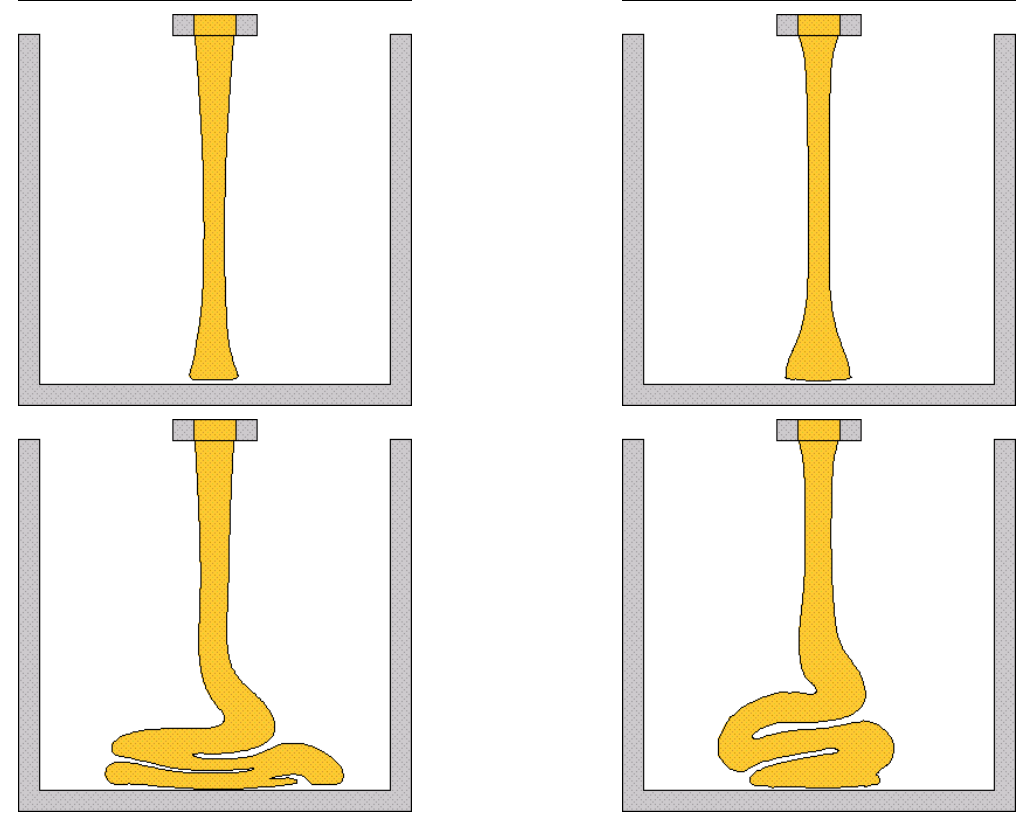

$t=0.14 \mathrm{~s}$
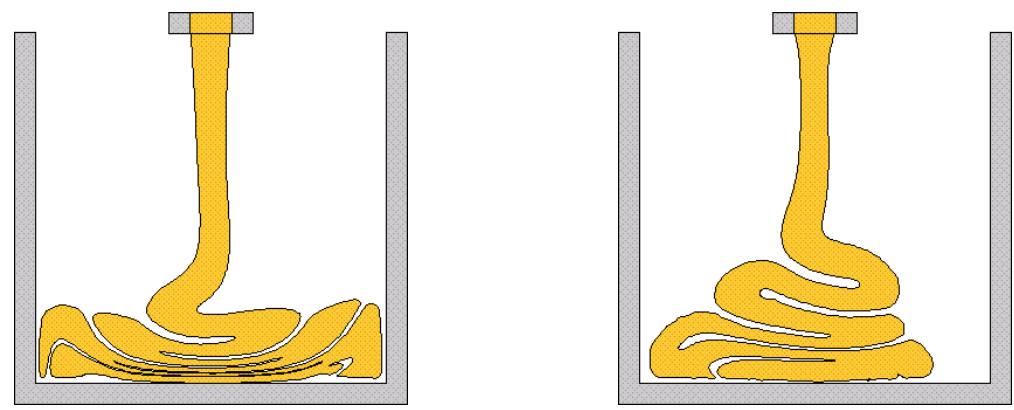

$t=0.30 \mathrm{~s}$
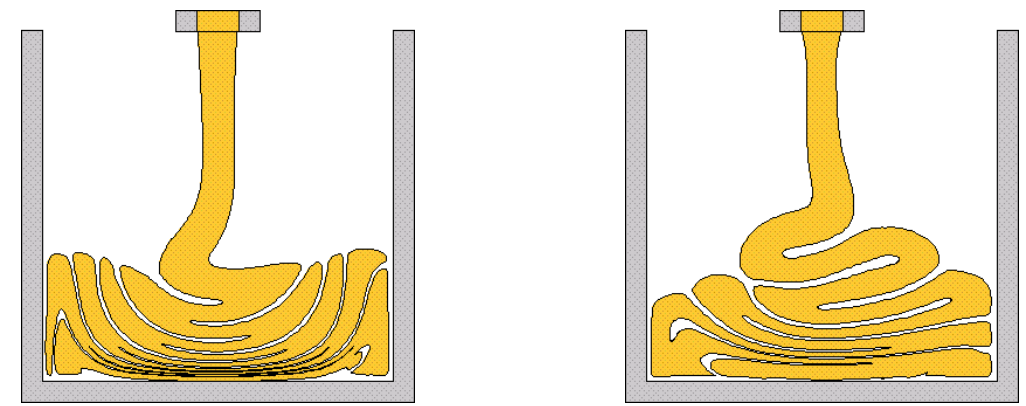

$t=0.44 \mathrm{~s}$

$t=0.62 \mathrm{~s}$

Figura 1.7: Simulação numérica do enchimento de um contêiner em diferentes tempos do escoamento. $R e=0.25, W e=1.5, \xi=0.2, \varepsilon=0.01$. (a esquerda) Modelo PTT - $\beta=0.1$. (a direita) Modelo PTT $-\beta=0.9$ 


\subsubsection{Simulação numérica do impacto de uma gota contra uma su- perfície rígida}

O impacto de uma gota de fluido contra uma superfície rígida é um fato presente em muitas aplicações industriais, tais como, impressoras a jato de tinta, pinturas, camadas por spray, etc.. Worthington foi um dos primeiros a investigar impactos sistematicamente, seu livro [90] contém fascinantes fotografias de fenômenos do imapcto de uma gota sólida e liquida em tanques liquidos. Estes fenômenos também atraem o público em geral, motivando os consumidores através de comerciais na TV que abusam desses fenômenos, como por exemplo, comerciais onde uma gota de detergente cai sobre uma louça; uma gota de hidratante ou shampoo caindo sobre a palma da mão, entre outros. Há mais de um século estes fenômenos vem sendo estudados e ainda hoje eles estão longe de serem compreendidos na sua totalidade por isso eles continuam atraindo muitos pesquisadores.

Motivados por esses fatos, nesta seção apresentamos resultados numéricos da simulação de uma gota de fluido incidindo perpendicularmente numa superfície plana rígida.

Os efeitos que podem ocorrem durante o impacto de uma gota com fluido contra uma superfície plana rígida são conhecidos como 'bouncing', 'spreading' e 'splashing'. Um esboço desses fenômenos é mostrado na figura 1.8 .

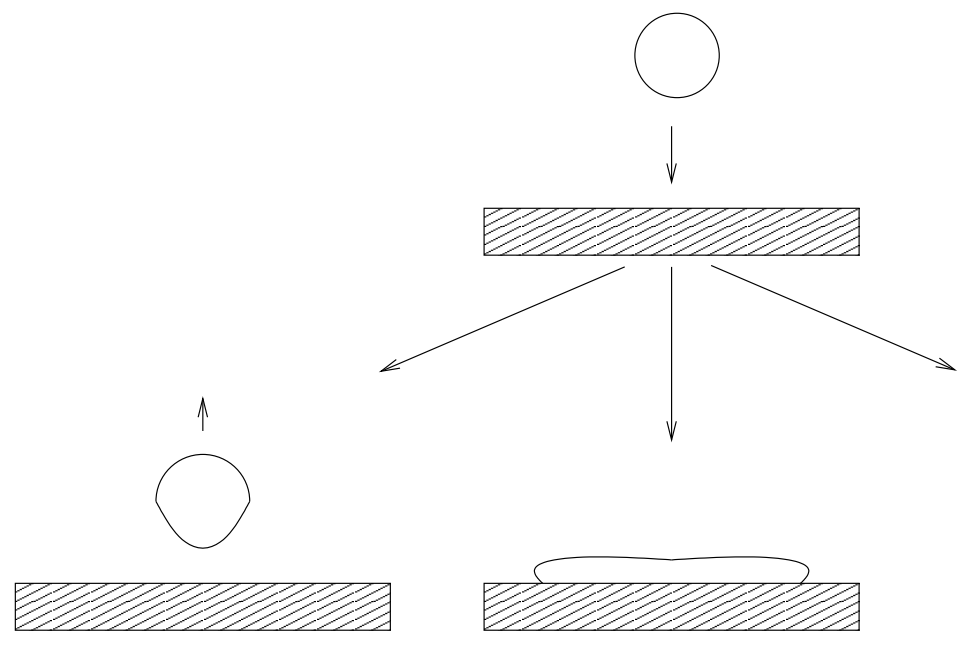

(a) (b)

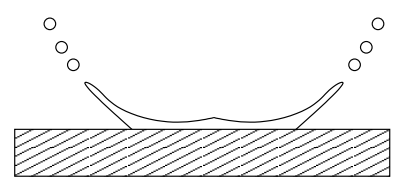

(c)

Figura 1.8: Tipos de fenômenos do impacto de uma gota com fluido contra uma superfície plana rígida: (a) 'bouncing'; (b) 'spreading'; (c) 'splashing'

Para mostrar que o método numérico apresentado nesse trabalho é capaz de simular o impacto de uma gota em uma placa rígida, foram realizadas várias simulações de uma gota esférica de fluido viscoelástico incidindo contra uma placa rígida e os resultados obtidos foram comparados com os resultados de uma gota esférica Newtoniana. Nessas simulações será adotada a mesma geometria e os dados utilizados estão dispostos na tabela 1.6.

Além de mostrar a diferença do escoamento produzido por uma gota viscoelástica e uma 
gota Newtoniana vamos também mostrar as diferenças no escoamento de duas gotas viscoelásticas. Desta forma, vamos variar o número de Weissenberg We e observar a sua influência no escoamento produzido por duas gotas viscoelásticas. Os dados utilizados nessas simulações estão dispostos na Tabela 1.7 .

\begin{tabular}{|l|l|}
\hline domínio & $5 \mathrm{~cm} \times 5 \mathrm{~cm}$ \\
\hline malha & $100 \times 100$ células \\
\hline altura do centro da gota até a placa rígida & $3 \mathrm{~cm}$ \\
\hline diâmetro da gota & $2 \mathrm{~cm}$ \\
\hline
\end{tabular}

Tabela 1.6: Modelagem do problema. Dados geométricos.

\begin{tabular}{|l|l|l|}
\hline Newtoniano e PTT: $R e=5$ & $W e=1$ & $W e=3$ \\
\hline \hline$U:$ velocidade inicial da gota & $-1 \mathrm{~ms}^{-1}$ & $-1 \mathrm{~ms}^{-1}$ \\
\hline$g_{x}:$ força gravitacional & $-9.81 \mathrm{~ms}^{-2}$ & $-9.81 \mathrm{~ms}^{-2}$ \\
\hline$\nu=\eta_{0} / \rho$ & $0.004 \mathrm{~m}^{2} \mathrm{~s}^{-1}$ & $0.004 \mathrm{~m}^{2} \mathrm{~s}^{-1}$ \\
\hline$F r$ & 2.2576 & 2.2576 \\
\hline$R e$ & 5 & 5 \\
\hline apenas para o modelo PTT & & \\
\hline $\boldsymbol{\lambda}$ & $\mathbf{0 . 0 2} \mathrm{s}$ & $\mathbf{0 . 0 6}$ \\
\hline $\boldsymbol{W} e$ & $\mathbf{1}$ & $\mathbf{3}$ \\
\hline$\varepsilon$ & 0.005 & 0.005 \\
\hline$\xi$ & 0.01 & 0.01 \\
\hline$\beta$ & 0.8 & 0.8 \\
\hline
\end{tabular}

Tabela 1.7: Dados utilizados na simulação do impacto normal das gotas esféricas de fluido Newtoniano e viscoelástico.

Os resultados dessas simulações são apresentados na figura 1.9. Na figura 1.9 a), podemos observar que a gota Newtoniana atinge a placa rígida e escoa radialmente mantendo sua forma côncava enquanto que as figuras 1.9 b) e 1.9 c) mostram que as gotas viscoelásticas apresentam o fenômeno 'bouncing'. No escoamento produzido pela gota viscoelástica com $W e=1$ pode ser observado três fases distintas. A primeira fase, entre o tempo que a gota atinge a placa rígida e o tempo $t=0.042 \mathrm{~s}$, o escoamento está associado a um valor positivo da velocidade radial $u$. Nesta fase o escoamento apresenta uma maior facilidade para escoar radialmente do que a gota Newtoniana. Na segunda fase ocorre no intervalo de tempo [0.042. 0.073], onde a velocidade radial $u$ têm uma inversão de sinal e a gota apresenta o efeito de uma contração. A partir do tempo $t=0.073$ s podemos notar que a gota passa por uma terceira fase caracterizada por uma nova inversao de sinal da velocidade radial $u$ o que faz com que a gota escoe radialmente adquirindo um formato semelhante ao formato da gota Newtoniana. O escoamento da gota com $W e=3$ apresenta efeitos semelhantes aos efeitos produzidos pela gota com $W e=1$ com a diferença que os efeitos são mais visiveis, isto é, o efeito da contração faz com que a gota adquira o formato próximo do esférico. A mudança de sinal na velocidade $u$ acontece várias 
vezes havendo uma diminuição na intensidade da velocidade o que faz com que o efeito da contração seja diminuido. 

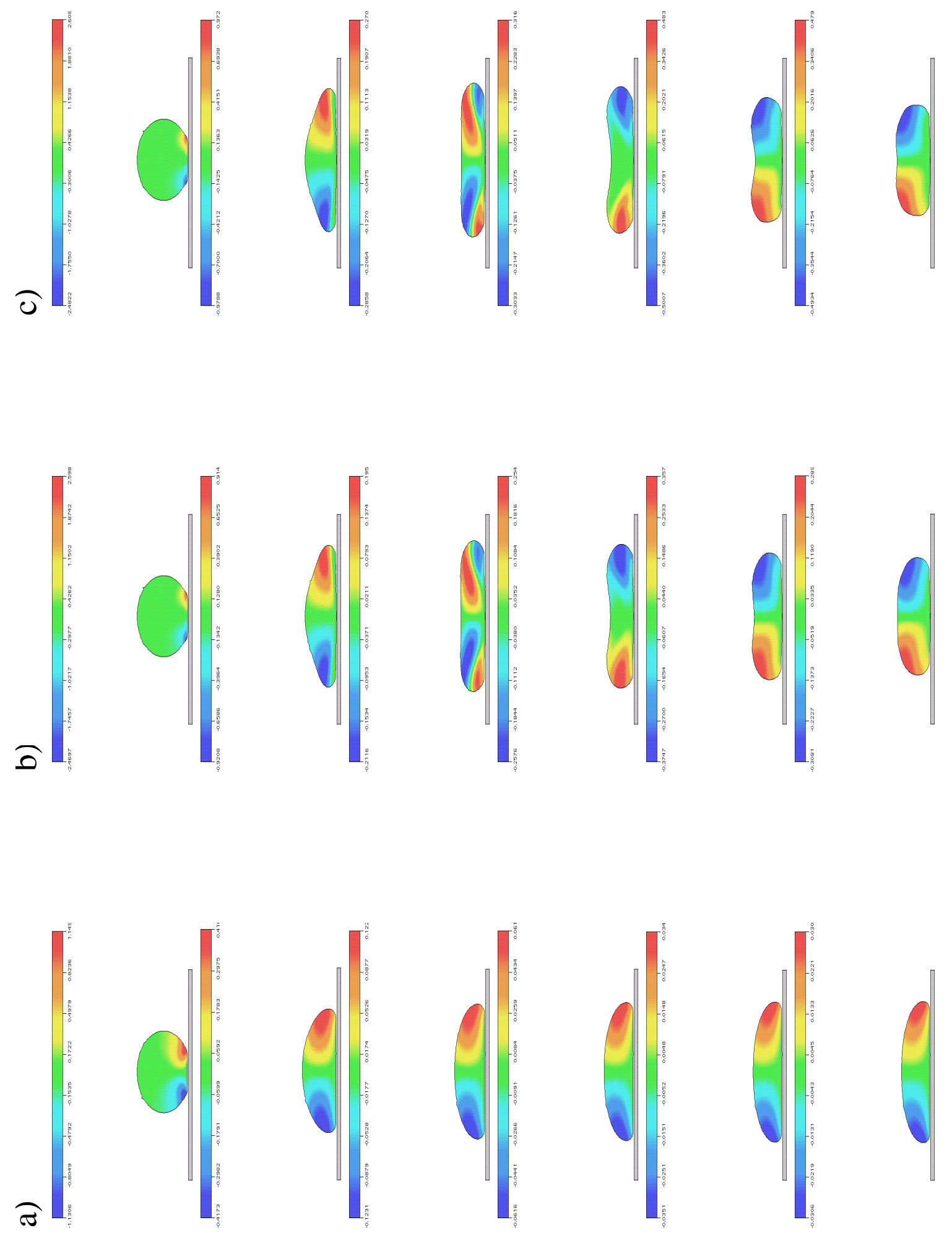

Figura 1.9: Perfil de velocidade $u$ em diferentes tempo: $t=0.027 \mathrm{~s}, t=0.035 \mathrm{~s}, t=0.042 \mathrm{~s}$, $t=0.047 \mathrm{~s}, t=0.055 \mathrm{~s}, t=0.060 \mathrm{~s}$. Coluna a) fluido Newtoniano: $R e=5$, Coluna b) fluido PTT: $W e=1$, e Coluna c) fluido PTT: $W e=3$. 

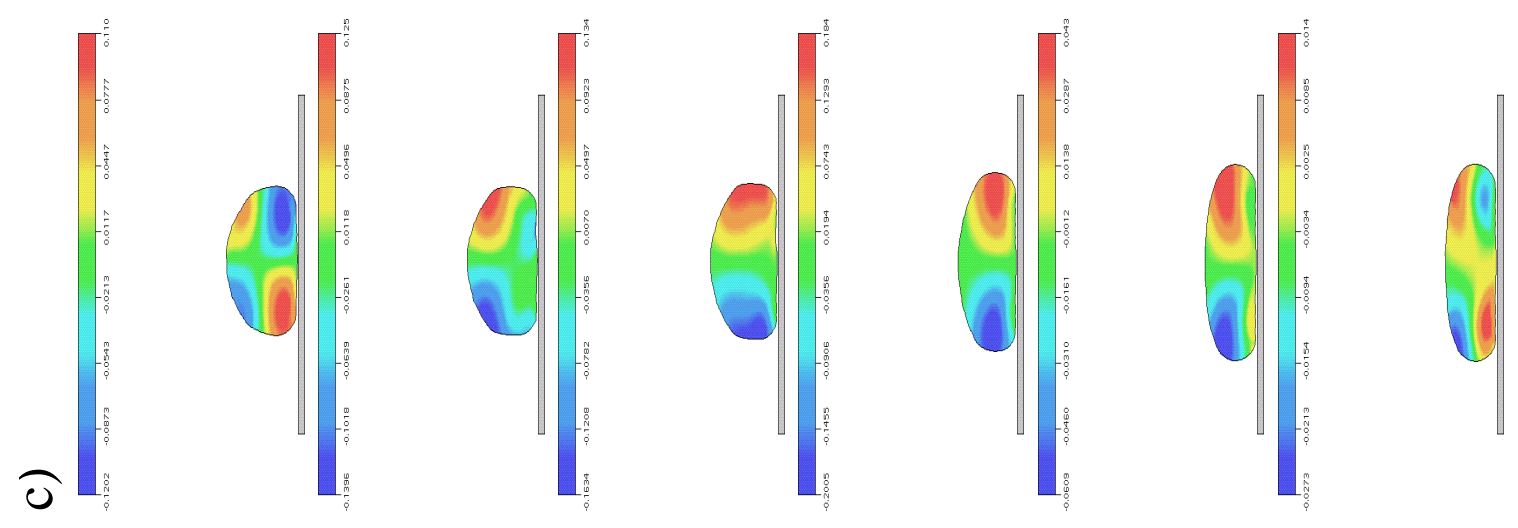

$\overbrace{}^{0}$
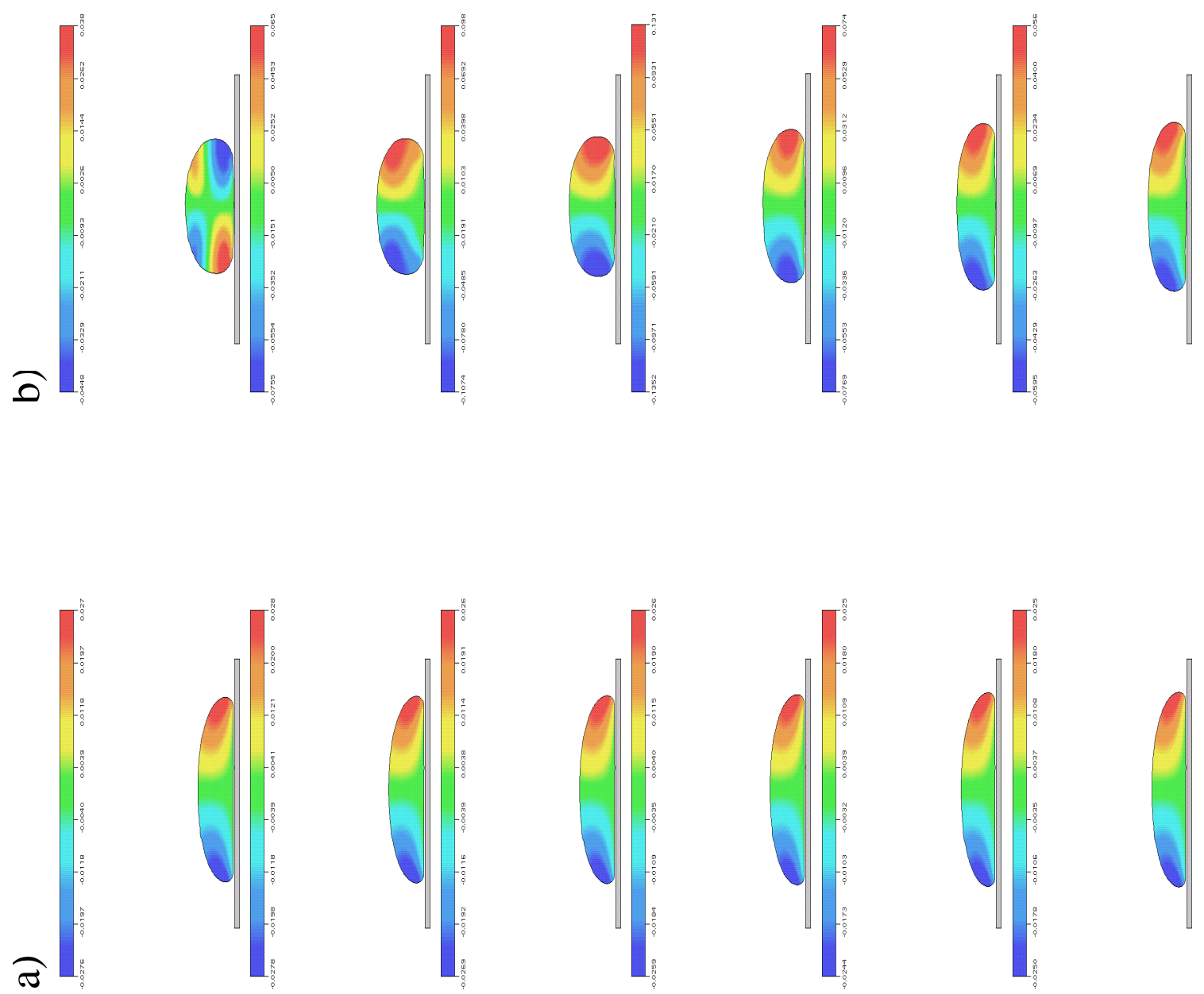

Figura 1.9. Continuação. Perfil de velocidade $u$ em diferentes tempos: $t=0.073 \mathrm{~s}, t=0.080 \mathrm{~s}$, $t=0.085 \mathrm{~s}, t=0.095 \mathrm{~s}, t=0.108 \mathrm{~s}, t=0.112 \mathrm{~s}$. Coluna a) fluido Newtoniano: $R e=5$, Coluna b) fluido PTT: $W e=1$, e Coluna c) fluido PTT: $W e=3$. 


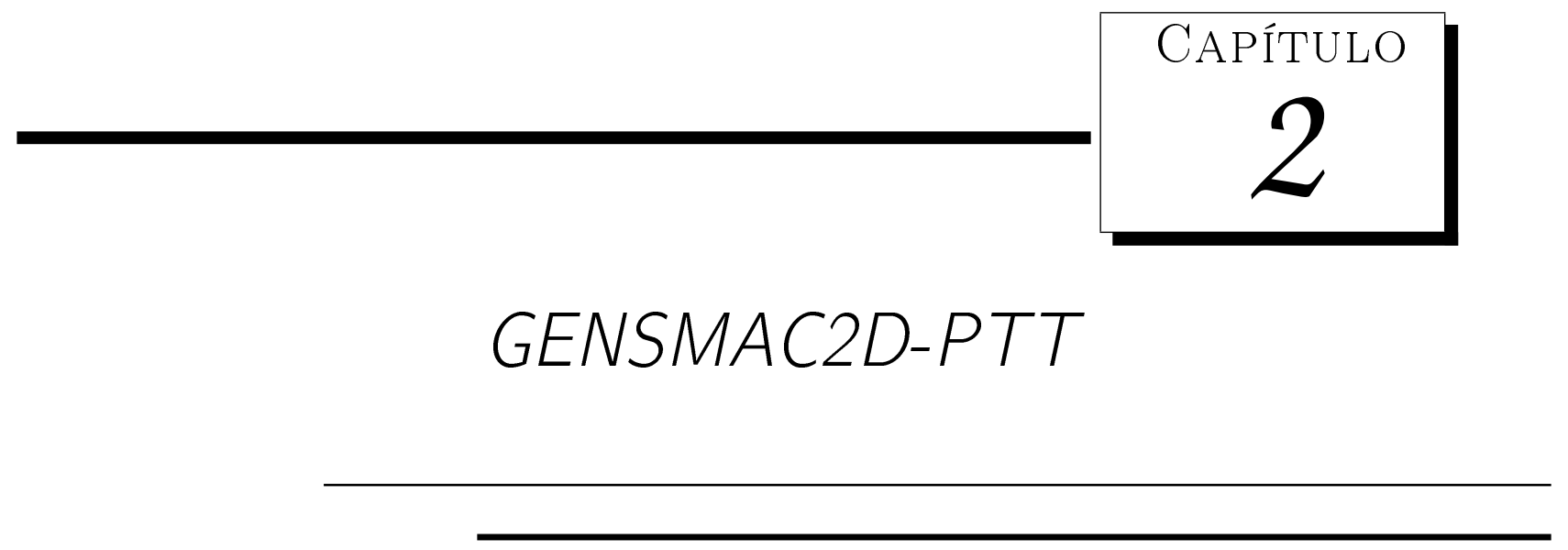

Este capítulo apresenta um método numérico para simular escoamentos viscoelásticos bidimensionais com superfícies livres em movimento usando a equação constitutiva PTT (Phan-Thien-Tanner). Inicialmente, descreve-se as equações básicas que governam escoamentos bidimensionais incompressíveis e isotérmicos descritos pelo modelo PTT. Em seguida, aborda-se a formulação matemática do problema para obtenção do método numérico GENSMAC2D-PTT. Para a adimensionalização das equações adota-se os seguintes números adimensionais: número de Reynolds $(R e)$, número de Weissenberg $(W e)$ e o número de Froude $(F r)$. O tratamento das condições de contorno é apresentado em detalhes e o método de solução, denominado GENSMAC2D-PTT, é discutido passo-a-passo. Finalmente, apresentam-se as aproximações das equações que descrevem o método numérico utilizando a técnica de diferenças finitas.

\subsection{O modelo PTT}

As equações básicas que governam escoamentos incompressíeis, isotérmicos descritos pelo modelo PTT são: equação da continuidade (conservação de massa), equação da conservação de quantidade de movimento e a equação constitutiva PTT que em notação tensorial são dadas por

$$
\begin{gathered}
\frac{\partial u_{i}}{\partial x_{i}}=0 \\
\rho \frac{\partial u_{i}}{\partial t}+\rho \frac{\partial u_{k} u_{i}}{\partial x_{k}}=-\frac{\partial p}{\partial x_{i}}+\frac{\partial \tau_{i k}}{\partial x_{k}}+\rho g_{i} \\
f\left(\tau_{k k}\right) \tau_{i j}+\lambda \stackrel{\square}{\tau i j}_{i j}=2 \eta D_{i j} .
\end{gathered}
$$

onde $t$ é o tempo, $x_{i}$ é o vetor espacial, $u_{i}$ é o vetor velocidade, $p$ é a pressão, $\rho$ é a massa específica do fluido, $g_{i}$ é o campo gravitacional, $\tau_{i k}$ é o tensor extra-stress, o qual se relaciona 
com as quantidades cinemáticas através da equação constitutiva (2.3),

$$
D_{i j}=\frac{1}{2}\left(\frac{\partial u_{i}}{\partial x_{j}}+\frac{\partial u_{j}}{\partial x_{i}}\right)
$$

é o tensor taxa de deformação, $\lambda$ é o tempo de relaxação do fluido, $\eta$ é um parâmetro do modelo PTT conhecido como viscosidade do fluido a baixas taxas de cizalhamento.

A função $f$, dependente do traço de $\tau_{i j}$, determina qual a forma do modelo PTT. Usualmente, a função $f$ assume uma das seguintes formas:

(i) Linear: $f\left(\tau_{k k}\right)=1+\frac{\lambda \varepsilon}{\eta} \tau_{k k}$

(ii) Quadrática: $f\left(\tau_{k k}\right)=1+\frac{\lambda \varepsilon}{\eta} \tau_{k k}+\frac{1}{2}\left(\frac{\lambda \varepsilon}{\eta} \tau_{k k}\right)^{2}$

(iii) Exponencial: $f\left(\tau_{k k}\right)=\exp \left(\frac{\lambda \varepsilon}{\eta} \tau_{k k}\right)$

A forma linear foi a forma proposta no paper original de Phan-Thien and Tanner [60] e portanto, neste trabalho vamos considerar o modelo PTT na forma linear, ou seja, a função $f$ será dada por (i).

O símbolo $(\cdot)$ representa a seguinte derivada convectiva:

$$
\stackrel{\square}{\tau}_{i j}=\frac{\partial \tau_{i j}}{\partial t}+\frac{\partial\left(u_{k} \tau_{i j}\right)}{\partial x_{k}}-\left(L_{i k}-\xi D_{i k}\right) \tau_{k j}-\left(L_{j k}-\xi D_{j k}\right) \tau_{k i},
$$

onde $L_{i j}=\partial u_{i} / \partial x_{j}$ é o tensor gradiente, a diferença $L_{i j}-\xi D_{i j}$ é chamada gradiente efetivo de velocidade [58], $\varepsilon$ e $\xi$ são parâmetros positivos do modelo PTT. O modelo SPTT (Simplified Phan-Thien-Tanner) é obtido fazendo $\xi=0$ [60] e obtém-se o modelo UCM (Upper-Convected-Maxwell) fazendo $\xi=0$ e $\varepsilon=0$ (ver [7]).

\subsection{Formulação do problema bidimensional II}

Para resolver as equações (2.1)-(2.3) empregamos a transformação EVSS (Elastic-Viscous Stress-Splitting) [64]. Essa formulação consiste em separar o tensor $\tau_{i j}$ em duas componentes, uma componente chamada de contribuição Newtoniana do fluido e a outra conhecida como contribuição não-Newtoniana. A decomposição de $\tau_{i j}$, dada pela equação (2.4), será muito importante para o desenvolvimento do método numérico porque adiciona na equação de conservação de quantidade de movimento (2.2) um termo difusivo que contribui para a convergência e estabilidade do método numérico a ser obtido. Assim, temos

$$
\tau_{i j}=2 \eta D_{i j}+S_{i j}
$$

onde $S_{i j}$ é o tensor não-Newtoniano, usualmente chamado de contribuição não-Newtoniana do tensor $\tau_{i j}$ e $D_{i j}$ é o tensor taxa de deformação. 
Substituindo a equação (2.4) nas equações (2.2) e (2.3) obtém-se:

$$
\begin{gathered}
\rho \frac{\partial u_{i}}{\partial t}+\rho \frac{\partial u_{k} u_{i}}{\partial x_{k}}=-\frac{\partial p}{\partial x_{i}}+\eta \frac{\partial}{\partial x_{k}}\left(\frac{\partial u_{i}}{\partial x_{k}}\right)+\frac{\partial S_{i k}}{\partial x_{k}}+\rho g_{i}, \\
f\left(S_{k k}\right) S_{i j}+\lambda \stackrel{\square}{S}_{i j}=2 \eta\left[1-f\left(S_{k k}\right)\right] D_{i j}-2 \lambda \eta \stackrel{\square}{D}_{i j},
\end{gathered}
$$

onde $f\left(S_{k k}\right)=1+\frac{\lambda \varepsilon}{\eta} S_{k k}$.

Portanto, deve-se resolver as equações $(2.1),(2.5)$ e (2.6) para as variáveis dependentes $u_{i}$, $p$ e $S_{i j}$

\subsection{Adimensionalização}

Nesse trabalho vamos considerar escoamentos cartesianos bidimensionais com $x_{i}=(x, y)$, $u_{i}=(u, v)$, onde as componentes nas direções $x$ e $y$ serão denominadas $u$ e $v$, respectivamente. Com o intuito de simplificar a notação, muitas vezes denotaremos apenas, por exempo, $u$ ao invés de $u(x, y, t)$. O tensor não-Newtoniano $S_{i j}$ será representado por

$$
S_{i j}=\left[\begin{array}{ll}
S^{x x} & S^{x y} \\
S^{x y} & S^{y y}
\end{array}\right]
$$

Os problemas de mecânica dos fluidos, em geral, são caracterizados por grandezas específicas, como velocidade, viscosidade, massa específica do escoamento, diamêtro de um tubo, etc. Essas grandezas dimensionais podem ser agrupadas em parâmetros adimensionais, que passam a caracterizar o escoamento. Neste trabalho, usaremos os seguintes parâmetros adimensionais: número de Reynolds, número de Weissenberg e número de Froude.

Para a adimensionalização das equações, as unidades escalares de comprimento, velocidade e massa específica serão denotadas por $L, U$ e $\rho$, respectivamente. A adimensionalização pode ser feita a partir das seguintes variáveis adimensionais:

$$
\begin{aligned}
& x=L \bar{x}, \quad u=U \bar{u}, \quad S^{x x}=\rho U^{2} \bar{S}^{x x}, \quad g_{x}=g \bar{g}_{x}, \\
& y=L \bar{y}, \quad v=U \bar{v}, \quad S^{x y}=\rho U^{2} \bar{S}^{x y}, g_{y}=g \bar{g}_{y}, \\
& t=(L / U) \bar{t}, \quad p=\rho U^{2} \bar{p}, \quad S^{y y}=\rho U^{2} \bar{S}^{y y},
\end{aligned}
$$

Introduzindo essas variáveis adimensionais nas equações (2.1), (2.5) e (2.6) obtemos as seguintes equações adimensionais:

$$
\begin{gathered}
\frac{\partial u}{\partial x}+\frac{\partial v}{\partial y}=0 \\
\frac{\partial u}{\partial t}=-\frac{\partial u^{2}}{\partial x}-\frac{\partial(v u)}{\partial y}-\frac{\partial p}{\partial x}+\frac{1}{R e}\left(\frac{\partial^{2} u}{\partial x^{2}}+\frac{\partial^{2} u}{\partial y^{2}}\right)+\frac{\partial S^{x x}}{\partial x}+\frac{\partial S^{x y}}{\partial y}+\frac{1}{F r^{2}} g_{x}
\end{gathered}
$$




$$
\begin{aligned}
& \frac{\partial v}{\partial t}=-\frac{\partial(u v)}{\partial x}-\frac{\partial v^{2}}{\partial y}-\frac{\partial p}{\partial y}+\frac{1}{R e}\left(\frac{\partial^{2} v}{\partial x^{2}}+\frac{\partial^{2} v}{\partial y^{2}}\right)+\frac{\partial S^{x y}}{\partial x}+\frac{\partial S^{y y}}{\partial y}+\frac{1}{F r^{2}} g_{y} \\
& \frac{\partial S^{x y}}{\partial t}=-f\left(S_{k k}\right) \frac{1}{W e} S^{x y}-\frac{\partial\left(u S^{x y}\right)}{\partial x}-\frac{\partial\left(v S^{x y}\right)}{\partial y}-\left[\left(\frac{\xi}{2}-1\right) \frac{\partial v}{\partial x}+\frac{\xi}{2} \frac{\partial u}{\partial y}\right] S^{x x} \\
& -\left[\left(\frac{\xi}{2}-1\right) \frac{\partial u}{\partial y}+\frac{\xi}{2} \frac{\partial v}{\partial x}\right] S^{y y}+\left[1-f\left(S_{k k}\right)\right] \frac{2}{R e W e} D^{x y} \\
& -\frac{2}{R e}\left\{\frac{\partial}{\partial t} D^{x y}+\frac{\partial\left(u D^{x y}\right)}{\partial x}+\frac{\partial\left(v D^{x y}\right)}{\partial y}+\left[\left(\frac{\xi}{2}-1\right) \frac{\partial v}{\partial x}+\frac{\xi}{2} \frac{\partial u}{\partial y}\right] D^{x x}\right. \\
& \left.+\left[\left(\frac{\xi}{2}-1\right) \frac{\partial u}{\partial y}+\frac{\xi}{2} \frac{\partial v}{\partial x}\right] D^{y y}\right\} \\
& \frac{\partial S^{x x}}{\partial t}=-f\left(S_{k k}\right) \frac{1}{W e} S^{x x}-\frac{\partial\left(u S^{x x}\right)}{\partial x}-\frac{\partial\left(v S^{x x}\right)}{\partial y}-\left[(\xi-2) \frac{\partial u}{\partial y}+\xi \frac{\partial v}{\partial x}\right] S^{x y} \\
& -2(\xi-1) \frac{\partial u}{\partial x} S^{x x}+\left[1-f\left(S_{k k}\right)\right] \frac{2}{R e W e} D^{x x} \\
& -\frac{2}{R e}\left\{\frac{\partial}{\partial t} D^{x x}+\frac{\partial\left(u D^{x x}\right)}{\partial x}+\frac{\partial\left(v D^{x x}\right)}{\partial y}+2(\xi-1)\left(D^{x x}\right)^{2}\right. \\
& \left.+\left[(\xi-2) \frac{\partial u}{\partial y}+\xi \frac{\partial v}{\partial x}\right] D^{x y}\right\} \\
& \frac{\partial S^{y y}}{\partial t}=-f\left(S_{k k}\right) \frac{1}{W e} S^{y y}-\frac{\partial\left(u S^{y y}\right)}{\partial x}-\frac{\partial\left(v S^{y y}\right)}{\partial y}-\left[(\xi-2) \frac{\partial v}{\partial x}+\xi \frac{\partial u}{\partial y}\right] S^{x y} \\
& -2(\xi-1) \frac{\partial v}{\partial y} S^{y y}+\left[1-f\left(S_{k k}\right)\right] \frac{2}{R e W e} D^{y y} \\
& -\frac{2}{R e}\left\{\frac{\partial}{\partial t} D^{y y}+\frac{\partial\left(u D^{y y}\right)}{\partial x}+\frac{\partial\left(v D^{y y}\right)}{\partial y}+2(\xi-1)\right)\left(D^{y y}\right)^{2} \\
& \left.+\left[(\xi-2) \frac{\partial v}{\partial x}+\xi \frac{\partial u}{\partial y}\right] D^{x y}\right\}
\end{aligned}
$$

onde $f\left(S_{k k}\right)=1+\varepsilon R e W e\left(S_{k k}\right) ; R e=\rho U L / \eta, W e=\lambda U / L$ and $F r=U / \sqrt{L g}$ denotam os números de Reynolds, de Weissenberg e de Froude, respectivamente.

\subsection{Condições de contorno}

Para resolver as equações (2.7)-(2.12) é necessário impor condições iniciais e de contorno.

A condição inicial para o escoamento é a distribuição espacial das variáveis dependentes no tempo $t=0$, enquanto a condição de contorno contêm informações físicas das variáveis dependentes em todo tempo $t$.

As condições iniciais do escoamento para as componentes da velocidade $u_{i}$ e para as com- 
ponentes do tensor não-Newtoniano $S_{i j}$ foram impostas como sendo nulas:

$$
u_{i}=0, \quad \tau_{i j}=0 \quad \text { e } \quad S_{i j}=0, \quad i, j=1,2,3 .
$$

Neste trabalho deve-se considerar condições de contorno para as variáveis dependentes nos seguintes tipos de contornos:

- contorno rígido: é a parede rígida do domínio do escoamento, ou seja, é uma geometria como contêiner, tubo, placa rígida, etc.;

- injetor: é a região do domínio do escoamento onde há entrada de fluido;

- ejetor: é a região do domínio do escoamento onde há saída de fluido;

- superfície livre: é a interface entre o fluido e a atmosfera.

Detalhes sobre cada uma dessas condições de contorno são dados a seguir.

\subsubsection{Contornos de entrada e saída de fluido}

Entende-se por contorno de injeção, ou simplesmente injetor, como a região do domínio do escoamento onde há entrada de fluido, e por contorno de ejeção, ou simplesmente ejetor, como a região do domínio do escoamento onde há saída de fluido. O cálculo da velocidade e do tensor extra-tensão sobre estes contornos são tratados como segue.

\section{- Contorno de injeção}

Na figura 2.1, I representa o contorno de injeção; $n$ representa a direção normal ao contorno de entrada de fluido; $m$ representa a direção tangencial ao contorno de entrada de fluido.

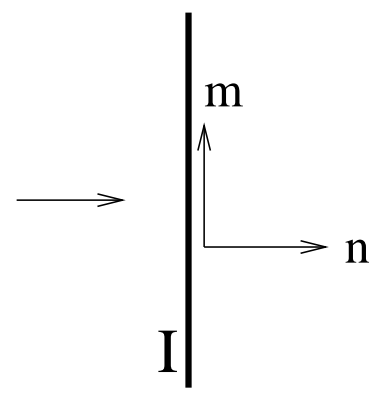

Figura 2.1: Esboço do contorno de entrada de fluido.

No injetor assume-se que as componentes da velocidade são dadas por

$$
u_{n}=U, \quad \text { e } \quad u_{m}=0
$$

ou seja, a componente normal da velocidade tem um valor não nulo prescrito no injetor, enquanto a componente tangencial da velocidade tem valor nulo. 
Para as componentes do tensor não-Newtoniano $S_{i j}$ foi empregada a estratégia adotada por Crochet [47] e Mompean \& Deville [50], a qual impõe-se o valor nulo para todas as componentes do tensor não-Newtoniano no injetor:

$$
S^{x x}=0, \quad S^{y y}=0, \quad \text { e } \quad S^{x y}=0 .
$$

\section{- Contorno de ejeção}

Na figura 2.2, $E$ representa o contorno de ejeção; $n$ representa a direção normal ao contorno de saída de fluido; $m$ representa a direção tangencial ao contorno de saída de fluido.

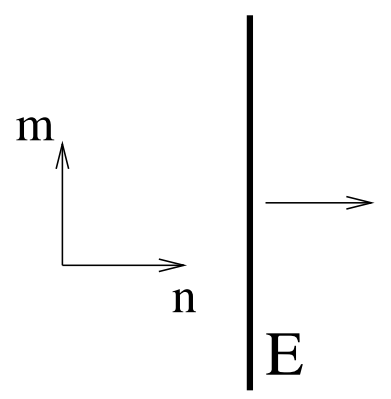

Figura 2.2: Esboço do contorno de saída de fluido.

No ejetor considera-se que não há variação nem da velocidade e nem das componentes do tensor não-Newtoniano na direção normal ao contorno. Então, impõem-se a condição homogênea de Neumann para as componentes da velocidade e do tensor não-Newtoniano neste contorno, ou seja,

$$
\begin{gathered}
\frac{\partial u_{n}}{\partial n}=0, \quad \frac{\partial u_{m}}{\partial n}=0 \\
\frac{\partial S^{x x}}{\partial n}=\frac{\partial S^{y y}}{\partial n}=\frac{\partial S^{x y}}{\partial n}=0 .
\end{gathered}
$$

\subsubsection{Condições na superfície livre}

Neste trabalho, considera-se escoamentos transientes com superfícies livres de um fluido viscoelástico movimentando-se numa atmosfera passiva, na qual pode-se tomar a pressão como sendo nula. Na ausência de efeitos de tensão superficial as componentes de tensão normal e tangencial devem ser contínuas sobre tais superfícies (ver [5]) e portanto,

$$
\begin{aligned}
& n_{i} \cdot\left(\sigma_{i j} \cdot n_{j}\right)=0, \\
& m_{i} \cdot\left(\sigma_{i j} \cdot n_{j}\right)=0, \quad i, j=1,2,
\end{aligned}
$$


onde $n_{i}$ denota o vetor unitário normal à superfície livre e $m_{i}$ denota o vetor unitário tangencial à superfície livre (ver a figura 2.3) e $\sigma_{i j}$ é o tensor total dado por

$$
\sigma_{i j}=-p \delta_{i j}+2 \eta D_{i j}+S_{i j}, \quad i, j=1,2 .
$$

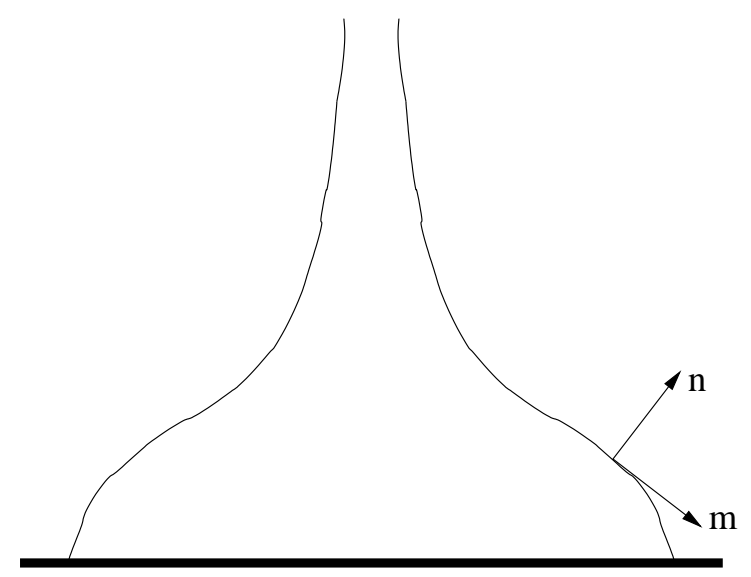

Figura 2.3: Exemplo de superfície livre do fluido e vetor normal e tangencial.

Usando coordenadas cartesianas bidimensionais e fazendo $n_{i}=\left(n_{x}, n_{y}\right)$ e $m_{i}=\left(n_{y}, \quad-n_{x}\right)$ a condição (2.13) pode ser escrita na forma adimensional como

$$
\begin{gathered}
p=\frac{2}{R e}\left[\left(\frac{\partial u}{\partial x} n_{x}{ }^{2}+\left(\frac{\partial u}{\partial y}+\frac{\partial v}{\partial x}\right) n_{x} n_{y}+\frac{\partial v}{\partial y} n_{y}{ }^{2}\right)\right]+\left(S^{x x} n_{x}{ }^{2}+2 S^{x y} n_{x} n_{y}+S^{y y} n_{y}{ }^{2}\right) \\
2\left(\frac{\partial u}{\partial x}-\frac{\partial v}{\partial y}\right) n_{x} n_{y}+\left(\frac{\partial u}{\partial y}+\frac{\partial v}{\partial x}\right)\left(n_{y}{ }^{2}-n_{x}{ }^{2}\right)+\left(S^{x x}-S^{y y}\right) n_{x} n_{y}+S^{x y}\left(n_{y}{ }^{2}-n_{x}{ }^{2}\right)=0 .
\end{gathered}
$$

As equações (2.14) e (2.15) representam a condição de contorno para as tensões na superfície livre.

\subsection{Cálculo do tensor não-Newtoniano em contornos rígi- dos}

Ao calcular as componentes do tensor não-Newtoniano $S_{i j}$ por meio das equações (2.10)-(2.12), vamos utilizar um método 'upwind' de alta ordem denominado CUBISTA (Convergent Universally Bounded Interpolation Scheme for the Treatment of Advection), desenvolvido por Alves et al. [1] para aproximar os termos convectivos. Este método necessita que os valores das variáveis sejam definidos em até dois nós vizinhos. Logo, para cálculos em pontos próximos aos contornos há a necessidade de se conhecer o valor do tensor não-Newtoniano nos contornos rígidos.

As componentes do tensor não-Newtoniano são calculadas no contorno rígido a partir 
das equações (2.11)-(2.10) seguindo as idéias de Tomé et al., 2002 [84]. Fazendo a mudança de variável

$$
S_{i j}=e^{-\frac{1}{W e} t} \tilde{S}_{i j}
$$

as equações (2.11)-(2.10) reduzem-se a

$$
\begin{aligned}
& \frac{\partial \tilde{S}^{x y}}{\partial t}=-\operatorname{Re} \varepsilon e^{-\frac{1}{W e} t}\left(\tilde{S}^{x x}+\tilde{S}^{y y}\right) \tilde{S}^{x y}-\frac{\partial\left(u \tilde{S}^{x y}\right)}{\partial x}-\frac{\partial\left(v \tilde{S}^{x y}\right)}{\partial y} \\
& -\left[\left(\frac{\xi}{2}-1\right) \frac{\partial v}{\partial x}+\frac{\xi}{2} \frac{\partial u}{\partial y}\right] \tilde{S}^{x x}-\left[\left(\frac{\xi}{2}-1\right) \frac{\partial u}{\partial y}+\frac{\xi}{2} \frac{\partial v}{\partial x}\right] \tilde{S}^{y y} \\
& -2 \varepsilon\left(\tilde{S}^{x x}+\tilde{S}^{y y}\right) D^{x y} \\
& -e^{\frac{1}{W e} t} \frac{2}{R e}\left\{\frac{\partial}{\partial t} D^{x y}+\frac{\partial\left(u D^{x y}\right)}{\partial x}+\frac{\partial\left(v D^{x y}\right)}{\partial y}+\left[\left(\frac{\xi}{2}-1\right) \frac{\partial v}{\partial x}+\frac{\xi}{2} \frac{\partial u}{\partial y}\right] D^{x x}\right. \\
& \left.+\left[\left(\frac{\xi}{2}-1\right) \frac{\partial u}{\partial y}+\frac{\xi}{2} \frac{\partial v}{\partial x}\right] D^{y y}\right\} \\
& \frac{\partial \tilde{S}^{x x}}{\partial t}=-\operatorname{Re} \frac{\varepsilon}{e}^{-\frac{1}{W e} t}\left(\tilde{S}^{x x}+\tilde{S}^{y y}\right) \tilde{S}^{x x}-\frac{\partial\left(u \tilde{S}^{x x}\right)}{\partial x}-\frac{\partial\left(v \tilde{S}^{x x}\right)}{\partial y} \\
& -\left[(\xi-2) \frac{\partial u}{\partial y}+\xi \frac{\partial v}{\partial x}\right] \tilde{S}^{x y}-2(\xi-1) \frac{\partial u}{\partial x} \tilde{S}^{x x} \\
& -2 \varepsilon\left(\tilde{S}^{x x}+\tilde{S}^{y y}\right) D^{x x} \\
& -e^{\frac{1}{W e} t} \frac{2}{R e}\left\{\frac{\partial}{\partial t} D^{x x}+\frac{\partial\left(u D^{x x}\right)}{\partial x}+\frac{\partial\left(v D^{x x}\right)}{\partial y}+2(\xi-1)\left(D^{x x}\right)^{2}\right. \\
& \left.+\left[(\xi-2) \frac{\partial u}{\partial y}+\xi \frac{\partial v}{\partial x}\right] D^{x y}\right\}
\end{aligned}
$$

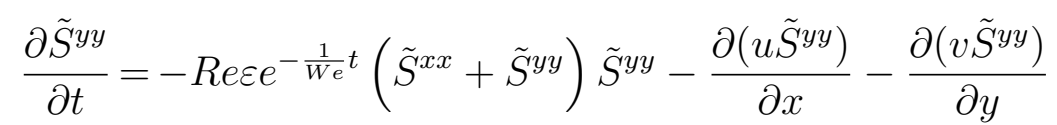

$$
\begin{aligned}
& -\left[(\xi-2) \frac{\partial v}{\partial x}+\xi \frac{\partial u}{\partial y}\right] \tilde{S}^{x y}-2(\xi-1) \frac{\partial v}{\partial y} \tilde{S}^{y y} \\
& -2 \varepsilon\left(\tilde{S}^{x x}+\tilde{S}^{y y}\right) D^{y y} \\
& -e^{\frac{1}{W e} t} \frac{2}{R e}\left\{\frac{\partial}{\partial t} D^{y y}+\frac{\partial\left(u D^{y y}\right)}{\partial x}+\frac{\partial\left(v D^{y y}\right)}{\partial y}+2(\xi-1)\right)\left(D^{y y}\right)^{2} \\
& \left.+\left[(\xi-2) \frac{\partial v}{\partial x}+\xi \frac{\partial u}{\partial y}\right] D^{x y}\right\} \text {. }
\end{aligned}
$$




\subsubsection{Contorno rígido paralelo ao eixo- $x$}

rigid boudary

Figura 2.4: Esboço do contorno rígido paralelo ao eixo-x.

Da condição não-escorregamento vem $\frac{\partial}{\partial x}=0 \Rightarrow \frac{\partial v}{\partial y}=0$. Assim, somente o termo $\frac{\partial u}{\partial y}$ é não-nulo. Logo, as equações (2.16)-(2.18) reduzem-se a

$$
\left\{\begin{aligned}
\frac{\partial \tilde{S}^{x y}}{\partial t}= & -e^{-\frac{1}{W e} t} \operatorname{Re\varepsilon }\left(\tilde{S}^{x x}+\tilde{S}^{y y}\right) \tilde{S}^{x y}-\frac{\xi}{2} \frac{\partial u}{\partial y} \tilde{S}^{x x}-\left(\frac{\xi}{2}-1\right) \frac{\partial u}{\partial y} \tilde{S}^{y y}, \\
& -\varepsilon\left(\tilde{S}^{x x}+\tilde{S}^{y y}\right) \frac{\partial u}{\partial y}-e^{\frac{1}{W e} t} \frac{1}{R e} \frac{\partial}{\partial t}\left(\frac{\partial u}{\partial y}\right) \\
\frac{\partial \tilde{S}^{x x}}{\partial t} & =-e^{-\frac{1}{W e} t} \operatorname{Re\varepsilon }\left(\tilde{S}^{x x}+\tilde{S}^{y y}\right) \tilde{S}^{x x}-(\xi-2) \frac{\partial u}{\partial y} \tilde{S}^{x y}-e^{\frac{1}{W e} t} \frac{1}{R e}(\xi-2)\left(\frac{\partial u}{\partial y}\right)^{2} \\
\frac{\partial \tilde{S}^{y y}}{\partial t} & =-e^{-\frac{1}{W e} t} \operatorname{Re\varepsilon }\left(\tilde{S}^{x x}+\tilde{S}^{y y}\right) \tilde{S}^{y y}-\xi \frac{\partial u}{\partial y} \tilde{S}^{x y}-e^{\frac{1}{W e} t} \frac{1}{R e} \xi\left(\frac{\partial u}{\partial y}\right)^{2} .
\end{aligned}\right.
$$

Os termos $\left(\tilde{S}^{x x}+\tilde{S}^{y y}\right) \tilde{S}^{m n}$ geram um sistema não-linear $3 \times 3(2.19)$. Esse sistema não-linear deve ser resolvido ao longo dos contornos rígidos paralelos ao eixo- $x$ em cada ciclo computacional. Isto faz com que esse novo método numérico para calcular o tensor nos contornos rígidos "custe muito caro computacionalmente". Portanto, como $\varepsilon e^{-\frac{1}{W e} t}<<1$, desprezaremos os termos $\left(\tilde{S}^{x x}+\tilde{S}^{y y}\right) \tilde{S}^{m n} \varepsilon e^{-\frac{1}{W e} t}$ do sistema (2.19) obtendo assim um sistema linear $3 \times 3$ como segue.

$$
\begin{aligned}
\frac{\partial \tilde{S}^{x y}}{\partial t} & =-\frac{\xi}{2} \frac{\partial u}{\partial y} \tilde{S}^{x x}-\left(\frac{\xi}{2}-1\right) \frac{\partial u}{\partial y} \tilde{S}^{y y}-\varepsilon\left(\tilde{S}^{x x}+\tilde{S}^{y y}\right) \frac{\partial u}{\partial y}-e^{\frac{1}{W e} t} \frac{1}{R e} \frac{\partial}{\partial t}\left(\frac{\partial u}{\partial y}\right) \\
\frac{\partial \tilde{S}^{x x}}{\partial t} & =-(\xi-2) \frac{\partial u}{\partial y} \tilde{S}^{x y}-e^{\frac{1}{W e} t} \frac{1}{R e}(\xi-2)\left(\frac{\partial u}{\partial y}\right)^{2}, \\
\frac{\partial \tilde{S}^{y y}}{\partial t} & =-\xi \frac{\partial u}{\partial y} \tilde{S}^{x y}-e^{\frac{1}{W e} t} \frac{1}{R e} \xi\left(\frac{\partial u}{\partial y}\right)^{2} .
\end{aligned}
$$

As equações (2.20)-(2.22) são resolvidas para as componentes $\tilde{S}^{x y}, \tilde{S}^{x x}$ e $\tilde{S}^{y y}$. Para obter expressões para essas componentes integra-se as equações $(2.20)-(2.22)$ no intervalo $[t, t+\delta t]$ 
como segue.

$$
\begin{aligned}
& \int_{t}^{t+\delta t} \frac{\partial \tilde{S}^{x y}(x, y, s)}{\partial s} d s=-\frac{\xi}{2} \int_{t}^{t+\delta t} \frac{\partial u(x, y, s)}{\partial y} \tilde{S}^{x x}(x, y, s) d s \\
&-\left(\frac{\xi}{2}-1\right) \int_{t}^{t+\delta t} \frac{\partial u(x, y, s)}{\partial y} \tilde{S}^{y y}(x, y, s) d s-\frac{1}{R e} \int_{t}^{t+\delta t} e^{\frac{1}{W e} s} \frac{\partial}{\partial s}\left(\frac{\partial u(x, y, s)}{\partial y}\right) d s \\
&-\varepsilon \int_{t}^{t+\delta t}\left(\tilde{S}^{x x}(x, y, s)+\tilde{S}^{y y}(x, y, s)\right) \frac{\partial u(x, y, s)}{\partial y} d s \\
& \int_{t}^{t+\delta t} \frac{\partial \tilde{S}^{x x}(x, y, s)}{\partial s} d s=-(\xi-2) \int_{t}^{t+\delta t} \frac{\partial u(x, y, s)}{\partial y} \tilde{S}^{x y}(x, y, s) d s \\
&-\frac{1}{R e}(\xi-2) \int_{t}^{t+\delta t} e^{\frac{1}{W e} s}\left(\frac{\partial u(x, y, s)}{\partial y}\right)^{2} d s \\
& \int_{t}^{t+\delta t} \frac{\partial \tilde{S}^{y y}(x, y, s)}{\partial s} d s=-\xi \int_{t}^{t+\delta t} \frac{\partial u(x, y, s)}{\partial y} \tilde{S}^{x y}(x, y, s) d s \\
&-\frac{1}{R e} \xi \int_{t}^{t+\delta t} e^{\frac{1}{W e} s}\left(\frac{\partial u(x, y, s)}{\partial y}\right)^{2} d s
\end{aligned}
$$
trapézios:

Para resolver as integrais da forma $\int_{t}^{t+\delta t} \frac{\partial u(x, y, s)}{\partial y} \tilde{S}^{m n}(x, y, s) d s$ usaremos a regra dos

$$
\int_{t}^{t+\delta t} \frac{\partial u(x, y, s)}{\partial s} \tilde{S}^{m n}(x, y, s) d s=\frac{\delta t}{2}\left(\frac{\partial u(x, y, t)}{\partial y} \tilde{S}^{m n}(x, y, t)+\frac{\partial u(x, y, t+\delta t)}{\partial y} \tilde{S}^{m n}(x, y, t+\delta t)\right)
$$

e pelo Teorema do valor médio para integrais tem-se:

$$
\int_{t}^{t+\delta t} e^{\frac{1}{W e} t}\left(\frac{\partial u(x, y, s)}{\partial y}\right)^{2} d s=W e e^{\frac{1}{W e} t}\left(e^{\frac{1}{W e} \delta t}-1\right)\left(\frac{\partial u\left(x, y, t^{*}\right)}{\partial y}\right)^{2}
$$

e utilizando integração por partes obtém-se

$$
\int_{t}^{t+\delta t} e^{\frac{1}{W e} s} \frac{\partial}{\partial s}\left(\frac{\partial u(x, y, s)}{\partial y}\right) d s=e^{\frac{1}{W e} t}\left[e^{\frac{1}{W e} \delta t} \frac{\partial u(x, y, t+\delta t)}{\partial y}-\left(e^{\frac{1}{W e} \delta t}-1\right) \frac{\partial u\left(x, y, t^{*}\right)}{\partial y}-\frac{\partial u(x, y, t)}{\partial y}\right] .
$$

onde $t^{*} \in(t, t+\delta t)$. 
Desta forma, resolvendo as integrais (2.23)-(2.25) usando (2.26)-(2.28) obtemos as equações

$$
\begin{gathered}
\tilde{S}^{x y(n+1)}=\tilde{S}^{x y}-\frac{\xi}{2} \frac{\delta t}{2}\left(\frac{\partial u}{\partial y} \tilde{S}^{x x}+\frac{\partial u^{(n+1)}}{\partial y} \tilde{S}^{x x(n+1)}\right)-\left(\frac{\xi}{2}-1\right) \frac{\delta t}{2}\left(\frac{\partial u}{\partial y} \tilde{S}^{y y}+\frac{\partial u^{(n+1)}}{\partial y} \tilde{S}^{y y(n+1)}\right) \\
-\frac{1}{R e} e^{\frac{1}{W e} t}\left[e^{\frac{1}{W e} \delta t} \frac{\partial u(x, y, t+\delta t)}{\partial y}-\left(e^{\frac{1}{W e} \delta t}-1\right) \frac{\partial u\left(x, y, t^{*}\right)}{\partial y}-\frac{\partial u(x, y, t)}{\partial y}\right] \\
-\varepsilon \frac{\delta t}{2}\left[\left(\tilde{S}^{x x}+\tilde{S}^{y y}\right) \frac{\partial u}{\partial y}+\left(\tilde{S}^{x x(n+1)}+\tilde{S}^{y y(n+1)}\right) \frac{\partial u^{(n+1)}}{\partial y}\right], \\
\tilde{S}^{x x(n+1)}=\tilde{S}^{x x}-(\xi-2) \frac{\delta t}{2}\left(\frac{\partial u}{\partial y} \tilde{S}^{x y}+\frac{\partial u^{(n+1)}}{\partial y} \tilde{S}^{x y(n+1)}\right)-(\xi-2) \frac{W e}{R e} e^{\frac{1}{W e} t\left(e^{\frac{1}{W e} \delta t}-1\right)\left(\frac{\partial u\left(x, y, t^{*}\right)}{\partial y}\right)^{2}}(2.30) \\
\tilde{S}^{y y(n+1)}=\tilde{S}^{y y}-\xi \frac{\delta t}{2}\left(\frac{\partial u}{\partial y} \tilde{S}^{x y}+\frac{\partial u^{(n+1)}}{\partial y} \tilde{S}^{x y(n+1)}\right)-\xi \frac{W e}{R e} e^{\frac{1}{W e} t\left(e^{\frac{1}{W e} \delta t}-1\right)\left(\frac{\partial u\left(x, y, t^{*}\right)}{\partial y}\right)^{2}} \cdot
\end{gathered}
$$

As equações (2.29)-(2.31) produzem um sistema $3 \times 3$ para as componenetes $\tilde{S}^{m n(n+1)}$. Multiplicando as equações $(2.29)-(2.31)$ por $e^{-\frac{1}{W e}(t+\delta t)}$ vamos obter equações para as componentes $S^{m n(n+1)}$ como segue.

$$
\begin{gathered}
S^{x y(n+1)}=e^{-\frac{1}{W e} \delta t} S^{x y}-\frac{\xi}{2} \frac{\delta t}{2}\left(\frac{\partial u}{\partial y} e^{-\frac{1}{W e} \delta t} S^{x x}+\frac{\partial u^{(n+1)}}{\partial y} S^{x x(n+1)}\right)-\left(\frac{\xi}{2}-1\right) \frac{\delta t}{2}\left(\frac{\partial u}{\partial y} e^{-\frac{1}{W e} \delta t} S^{y y}\right. \\
\left.+\frac{\partial u^{(n+1)}}{\partial y} S^{y y(n+1)}\right)-\frac{1}{R e}\left[\frac{\partial u(x, y, t+\delta t)}{\partial y}-\left(1-e^{-\frac{1}{W e} \delta t}\right) \frac{\partial u\left(x, y, t^{*}\right)}{\partial y}-e^{-\frac{1}{W e} \delta t} \frac{\partial u(x, y, t)}{\partial y}\right] \\
-\varepsilon \frac{\delta t}{2}\left[e^{-\frac{1}{W e} \delta t}\left(S^{x x}+S^{y y}\right) \frac{\partial u}{\partial y}+\left(S^{x x(n+1)}+S^{y y(n+1)}\right) \frac{\partial u^{(n+1)}}{\partial y}\right] \\
S^{x x(n+1)}=e^{-\frac{1}{W e} \delta t} S^{x x}-(\xi-2) \frac{\delta t}{2}\left(\frac{\partial u}{\partial y} e^{-\frac{1}{W e} \delta t} S^{x y}+\frac{\partial u^{(n+1)}}{\partial y} S^{x y(n+1)}\right) \\
-(\xi-2) \frac{W e}{R e}\left(1-e^{-\frac{1}{W e} \delta t}\right)\left(\frac{\partial u\left(x, y, t^{*}\right)}{\partial y}\right)^{2}
\end{gathered}
$$




$$
\begin{aligned}
S^{y y(n+1)}=e^{-\frac{1}{W e} \delta t} S^{y y} & -\xi \frac{\delta t}{2}\left(\frac{\partial u}{\partial y} e^{-\frac{1}{W e} \delta t} S^{x y}+\frac{\partial u^{(n+1)}}{\partial y} S^{x y(n+1)}\right) \\
& -\xi \frac{W e}{R e}\left(1-e^{-\frac{1}{W e} \delta t}\right)\left(\frac{\partial u\left(x, y, t^{*}\right)}{\partial y}\right)^{2} .
\end{aligned}
$$

Note que, ambas as componentes $S^{x x}$ e $S^{y y}$ dependem da componente $S^{x y}$ em $t+\delta t$ (ver equações (2.33) e (2.34)), enquanto a componente $S^{x y}$ depende das componentes $S^{x x}$ e $S^{y y}$ em $t+\delta t$ (ver equação $(2.32)$ ).

Portanto, substituindo as equações (2.33) e (2.34) na equação (2.32) obtém-se uma expressão para a componente $S^{x y}$ que não depende das componentes $S^{x x}$ e $S^{y y}$ em $t+\delta t$ como vemos a seguir:

$$
\begin{gathered}
S^{x y}(x, y, t+\delta t)=\left\{e^{-\frac{1}{W e} \delta t} S^{x y}(x, y, t)-e^{-\frac{1}{W e} \delta t} \frac{\delta t}{2}\left[\left(\frac{\xi}{2}+\varepsilon\right) S^{x x}+\left(\frac{\xi}{2}+\varepsilon-1\right) S^{y y}\right] \frac{\partial u}{\partial y}\right. \\
\left.-\frac{1}{R e}\left[\frac{\partial u(x, y, t+\delta t)}{\partial y}-\left(1-e^{-\frac{1}{W e} \delta t}\right) \frac{\partial u\left(x, y, t^{*}\right)}{\partial y}-e^{-\frac{1}{W e} \delta t} \frac{\partial u(x, y, t)}{\partial y}\right]\right\} / \operatorname{Den} S^{x y},
\end{gathered}
$$

onde $\quad \operatorname{Den} S^{x y}=1-\left[\xi^{2}-2 \xi+2(\xi-1) \varepsilon\right] \frac{\delta t^{2}}{4}\left(\frac{\partial u(x, y, t+\delta t)}{\partial y}\right)^{2}$.

Logo, primeiramente calcula-se $S^{x y}$ usando a equação (2.35) e depois calcula-se $S^{x x}$ e $S^{y y}$ das equações (2.33) e (2.34), respectivamente.

\subsubsection{Contorno rígido paralelo ao eixo-y}

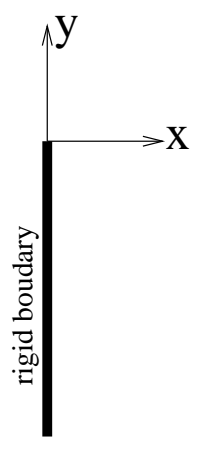

Figura 2.5: Esboço do contorno rígido paralelo ao eixo-y.

Da condição não-escorregamento vem $\frac{\partial}{\partial y}=0 \Rightarrow \frac{\partial u}{\partial x}=0$. Assim, somente o termo $\frac{\partial v}{\partial x}$ é 
não-nulo e as equações (2.16)-(2.18) reduzem-se a

$$
\begin{aligned}
& \frac{\partial \tilde{S}^{x y}}{\partial t}=-e^{-\frac{1}{W e} t} \operatorname{Re\varepsilon }\left(\tilde{S}^{x x}+\tilde{S}^{y y}\right) \tilde{S}^{x y}-\left(\frac{\xi}{2}-1\right) \frac{\partial v}{\partial x} \tilde{S}^{x x}-\frac{\xi}{2} \frac{\partial v}{\partial x} \tilde{S}^{y y}, \\
&-\varepsilon\left(\tilde{S}^{x x}+\tilde{S}^{y y}\right) \frac{\partial v}{\partial x}-e^{\frac{1}{W e} t} \frac{1}{R e} \frac{\partial}{\partial t}\left(\frac{\partial v}{\partial x}\right), \\
& \frac{\partial \tilde{S}^{x x}}{\partial t}=-e^{-\frac{1}{W e} t} \operatorname{Re\varepsilon }\left(\tilde{S}^{x x}+\tilde{S}^{y y}\right) \tilde{S}^{x x}-\xi \frac{\partial v}{\partial x} \tilde{S}^{x y}-e^{\frac{1}{W e} t} \frac{1}{R e} \xi\left(\frac{\partial v}{\partial x}\right)^{2} \\
& \frac{\partial \tilde{S}^{y y}}{\partial t}=-e^{-\frac{1}{W e} t} \operatorname{Re\varepsilon }\left(\tilde{S}^{x x}+\tilde{S}^{y y}\right) \tilde{S}^{y y}-(\xi-2) \frac{\partial v}{\partial x} \tilde{S}^{x y}-e^{\frac{1}{W e} t} \frac{1}{R e}(\xi-2)\left(\frac{\partial v}{\partial x}\right)^{2} .
\end{aligned}
$$

O cálculo de $S^{x y}, S^{x x}, S^{y y}$ em contornos rígidos paralelos ao eixo-y segue análogo ao caso de contornos rígidos paralelos ao eixo $x$. Nesse caso, pode-se mostrar que as componentes do tensor não-Newtoniano em contornos rígidos paralelos ao eixo $y$ são dadas por:

$$
\begin{aligned}
& S^{x y}(x, y, t+\delta t)=\left\{e^{-\frac{1}{W e} \delta t} S^{x y}(x, y, t)-e^{-\frac{1}{W e} \delta t} \frac{\delta t}{2}\left[\left(\frac{\xi}{2}+\varepsilon-1\right) S^{x x}+\left(\frac{\xi}{2}+\varepsilon\right) S^{y y}\right] \frac{\partial v}{\partial x}\right. \\
& \left.-\frac{1}{R e}\left[\frac{\partial v(x, y, t+\delta t)}{\partial x}-\left(1-e^{-\frac{1}{W e} \delta t}\right) \frac{\partial v\left(x, y, t^{*}\right)}{\partial x}-e^{-\frac{1}{W e} \delta t} \frac{\partial v(x, y, t)}{\partial x}\right]\right\} / \operatorname{Den} S^{x y} \\
& S^{x x(n+1)}=e^{-\frac{1}{W e} \delta t} S^{x x}-\xi \frac{\delta t}{2}\left(\frac{\partial v}{\partial x} e^{-\frac{1}{W e} \delta t} S^{x y}+\frac{\partial v^{(n+1)}}{\partial x} S^{x y(n+1)}\right)-\xi \frac{W e}{R e}\left(1-e^{-\frac{1}{W e} \delta t}\right)\left(\frac{\partial v\left(x, y, t^{*}\right)}{\partial x}\right)^{2}, \\
& S^{y y(n+1)}=e^{-\frac{1}{W e} \delta t} S^{y y} \quad-(\xi-2) \frac{\delta t}{2}\left(\frac{\partial v}{\partial x} e^{-\frac{1}{W e} \delta t} S^{x y}+\frac{\partial v^{(n+1)}}{\partial x} S^{x y(n+1)}\right) \\
& -(\xi-2) \frac{W e}{R e}\left(1-e^{-\frac{1}{W e} \delta t}\right)\left(\frac{\partial v\left(x, y, t^{*}\right)}{\partial x}\right)^{2}
\end{aligned}
$$




\subsection{Método de solução}

Para resolver as equações (2.7)-(2.12) empregamos o seguinte procedimento. Assumimos que num dado instante de tempo $t_{n}$ o vetor velocidade $u_{i}\left(x_{k}, t_{n}\right)$ e o tensor não-Newtoniano $S_{i j}\left(x_{k}, t_{n}\right)$ são conhecidos e são dadas condições de contorno para a velocidade e pressão. Para calcular o vetor velocidade, o campo de pressão e o tensor não-Newtoniano no tempo $t_{n+1}=$ $t_{n}+\delta t$, ou seja, $u_{i}\left(x_{k}, t_{n+1}\right), p\left(x_{k}, t_{n+1}\right)$ e $S_{i j}\left(x_{k}, t_{n+1}\right)$, procedemos como segue:

Passo 1: Seja $\tilde{p}\left(x_{k}, t_{n}\right)$ um campo de pressão arbitrário que satisfaz a condição (2.13) na superfície livre.

Passo 2: Calcular o campo de velocidade intermediário $\widetilde{u}_{i}\left(x_{k}, t_{n+1}\right)$ por meio de

$$
\frac{\partial \widetilde{u}_{i}}{\partial t}=\frac{\partial\left(u_{k} u_{i}\right)}{\partial x_{k}}-\frac{\partial \widetilde{p}}{\partial x_{i}}+\frac{1}{R e} \frac{\partial}{\partial x_{k}}\left(\frac{\partial u_{i}}{\partial x_{k}}\right)+\frac{\partial S_{i k}}{\partial x_{k}}+\frac{1}{F r^{2}} g_{i},
$$

com $\widetilde{u}_{i}\left(x_{k}, t_{n}\right)=u_{i}\left(x_{k}, t_{n}\right)$ e utilizando as mesmas condições de contorno da velocidade $u_{i}\left(x_{k}, t_{n}\right)$. Pode-se mostrar que $\widetilde{u}_{i}\left(x_{k}, t_{n+1}\right)$ tem a vorticidade correta no tempo $t_{n+1}$.

Passo 3: Resolver a equação de Poisson

$$
\nabla^{2} \psi\left(x_{k}, t_{n+1}\right)=\nabla \cdot \widetilde{u}_{i}\left(x_{k}, t_{n+1}\right)
$$

Passo 4: Calcular a velocidade final dada por

$$
u_{i}\left(x_{k}, t_{n+1}\right)=\widetilde{u}_{i}\left(x_{k}, t_{n+1}\right)-\frac{\partial \psi\left(x_{k}, t_{n+1}\right)}{\partial x_{i}}
$$

Passo 5: Calcular a pressão:

$$
p\left(x_{k}, t_{n+1}\right)=\tilde{p}\left(x_{k}, t_{n}\right)+\frac{\psi\left(x_{k}, t_{n+1}\right)}{\delta t} .
$$

Passo 6: Calcular o tensor não-Newtoniano $S_{i j}\left(x_{k}, t_{n+1}\right)$

(a) calcule o the tensor não-Newtoniano nos contornos: injetor, ejetor e contornos rigídos conforme especificado nas Seções 1.4 e 1.5 .

(b) calcule $S_{i j}\left(x_{k}, t_{n+1}\right)$ por meio da equação

$$
f\left(S_{k k}\right) S_{i j}+W e \stackrel{\square}{S}_{i j}=\frac{2}{R e}\left[1-f\left(S_{k k}\right)\right] D_{i j}-2 \frac{W e}{R e} \stackrel{\square}{D}_{i j}
$$

onde $f\left(S_{k k}\right)=1+\varepsilon R e W e S_{k k}$, ou seja, por meio das equações (2.10)-(2.12). 
Passo 7: Atualizar as posições das partículas marcadoras: o último passo no ciclo computacional é mover as partículas marcadoras para as suas novas posições. esse passo é efetuado resolvendo a equação

$$
\frac{d x_{i}}{d t}=u_{i}
$$

para cada partícula marcadora. A superfície livre do fluido é então definida por uma lista contendo as coordenadas dessas partículas marcadoras. A visualização da superfície livre é obtida conectando, localmente, essas partículas por retas.

\subsection{Aproximação das equações por diferenças finitas}

As equações que descrevem o método numérico apresentado na seção 2.6 serão resolvidas pela técnica de diferenças finitas. A célula computacional adotada tem dimensões $\delta x \times \delta y$ e compõe uma malha conhecida como malha deslocada. As variáveis do problema estão localizadas nesta malha da seguinte forma: a pressão $p$, a função potencial $\psi$, as componentes do tensor não-Newtoniano $S^{x x}, S^{y y}$ e $S^{x y}$ e as componentes do tensor taxa de deformação $D^{x x}$, $D^{y y}$ e $D^{x y}$ são calculadas no centro da célula computacional, ou seja, no ponto $(i, j)$ enquanto as componentes da velocidade $u$ e $v$ são cálculadas no centro das seguintes arestas da célula computacional, isto é, nos pontos: $\left(i+\frac{1}{2}, j\right)$, e $\left(i, j+\frac{1}{2}\right)$, respectivamente (ver figura 2.6).

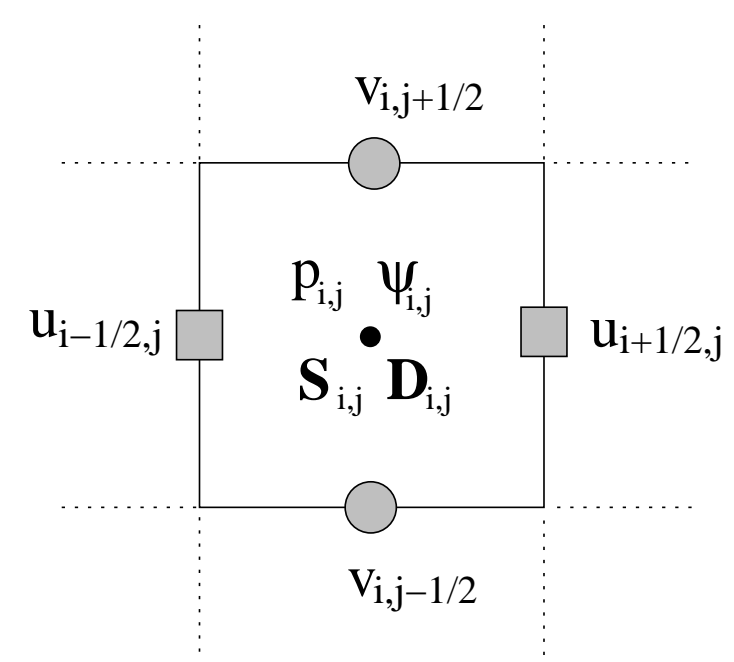

Figura 2.6: Célula computacional utilizada em GENSMAC2D-PTT.

\subsubsection{Classificação das Células}

Como o fluido está em movimento é necessário fazer uma classificação das células da malha para identificar se as mesmas contém fluido, estão no contorno rígido ou são células da superfície livre. Como o fluido está continuamente em movimento, um procedimento para identificar a região que contém fluido e a região da superfície livre é empregado. A figura 2.7 ilustra a classificação das células em um dado instante para um escoamento em um canal bidimensional. As células na malha são nomeadas como segue. 


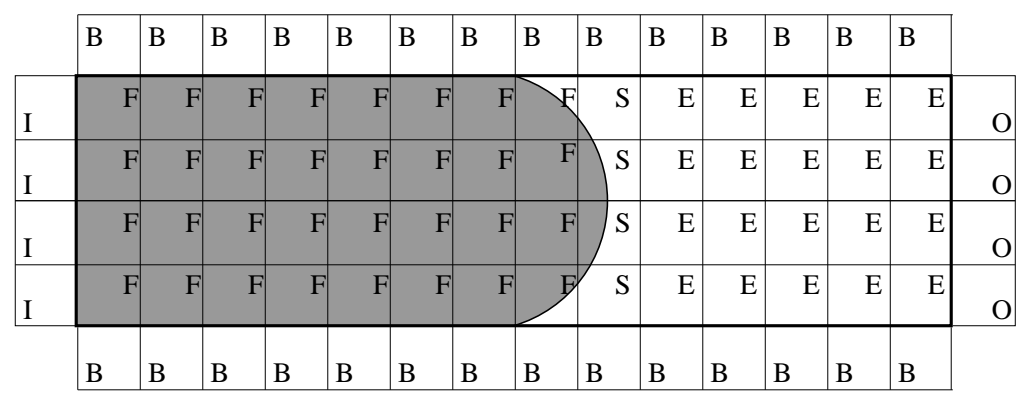

Figura 2.7: Tipos de células do domínio computacional.

$\triangleright$ células vazias (E): são células que não contém fluido;

$\triangleright$ células cheias (F): são células cheias de fluido e não possuem nenhuma face em contato com células vazias;

$\triangleright$ células de superfíie (S): são células que contém fluido e possuem pelo menos uma face em contato com células vazias;

$\triangleright$ células de contorno rígido (B): são células que definem um contorno rígido de maneira que as condições de contorno possam ser impostas;

$\triangleright$ células de injetor (I): são células que definem a entrada de fluido;

$\triangleright$ células de ejetor $(\mathrm{O})$ : são células que definem a saída de fluido.

\subsubsection{Aproximação das equações básicas}

A derivada temporal na equação (2.40) é aproximada pelo método de Euler explicito, o qual é de primeira ordem. O gradiente de pressão e os termos lineares da equação de quantidade de movimento são aproximados por diferenças centrais. Para os termos convectivos emprega-se o método 'upwind' de alta ordem CUBISTA [1]- Convergent and Universally Bounded Interpolation Scheme for the Treatment of Advecion. Os termos envolvendo o divergente do tensor não-Newtoniano são aproximados por diferenças centrais. Segue abaixo as aproximações das equações básicas.

$$
\begin{aligned}
\tilde{u}_{i+\frac{1}{2}, j}^{(n+1)} & =\tilde{u}_{i+\frac{1}{2}, j}-\operatorname{conv}\left(u^{2}\right)_{i+\frac{1}{2}, j}-\operatorname{conv}(v u)_{i+\frac{1}{2}, j}-\frac{\tilde{p}_{i+1, j}-\tilde{p}_{i, j}}{\delta x}+\frac{1}{R e}\left(\frac{u_{i+\frac{3}{2}, j}-2 u_{i+\frac{1}{2}, j}+u_{i-\frac{1}{2}, j}}{\delta x^{2}}\right. \\
& \left.+\frac{u_{i+\frac{1}{2}, j+1}-2 u_{i+\frac{1}{2}, j}+u_{i+\frac{1}{2}, j-1}}{\delta y^{2}}\right)+\frac{S_{i+1, j}^{x x}-S_{i, j}^{x x}}{\delta x}+\frac{S_{i+\frac{1}{2}, j+\frac{1}{2}}^{x y}-S_{i+\frac{1}{2}, j-\frac{1}{2}}^{x y}+\frac{1}{F r^{2}} g_{x},}{\delta y} \\
\tilde{v}_{i, j+\frac{1}{2}}^{(n+1)}= & \tilde{v}_{i, j+\frac{1}{2}}-\operatorname{conv}(u v)_{i, j+\frac{1}{2}}-\operatorname{conv}\left(v^{2}\right)_{i, j+\frac{1}{2}}-\frac{\tilde{p}_{i, j+1}-\tilde{p}_{i, j}}{\delta y}+\frac{1}{R e}\left(\frac{v_{i+1, j+\frac{1}{2}}-2 v_{i, j+\frac{1}{2}}+v_{i-1, j+\frac{1}{2}}}{\delta x^{2}}\right. \\
& \left.+\frac{v_{i+1, j+\frac{3}{2}}-2 v_{i, j+\frac{1}{2}}+v_{i, j-\frac{1}{2}}}{\delta y^{2}}\right)+\frac{S_{i+\frac{1}{2}, j+\frac{1}{2}}^{x y}-S_{i-\frac{1}{2}, j+\frac{1}{2}}^{x y}}{\delta x}+\frac{S_{i, j+1}^{y y}-S_{i, j-1}^{y y}}{\delta y}+\frac{1}{F r^{2}} g_{y},
\end{aligned}
$$


onde os temos como $S_{i+\frac{1}{2}, j+\frac{1}{2}}^{x y}$ são obtidos pela média dos quatro valores mais próximos, ou seja,

$$
S_{i+\frac{1}{2}, j+\frac{1}{2}}^{x y}:=\frac{\left(S_{i, j}^{x y}+S_{i+1, j}^{x y}+S_{i, j+1}^{x y}+S_{i+1, j+1}^{x y}\right)}{4} .
$$

os termos $\operatorname{conv}\left(u^{2}\right)_{i+\frac{1}{2}, j}, \cdots, \operatorname{conv}\left(v^{2}\right)_{i, j+\frac{1}{2}}$ representam os termos convectivos da equação $(2.40)$.

Logo, a discretização da equação de conservação de quantidade de movimento é de segunda ordem no espaço e primeira ordem no tempo.

A equação de Poisson, equação (2.41), é discretizada no centro da célula utilizando-se o operador Laplaciano discreto com 5 pontos, a qual fornece a seguinte equação de diferenças

$$
\frac{\psi_{i+1, j}-2 \psi_{i, j}+\psi_{i-1, j}}{\delta x^{2}}+\frac{\psi_{i, j+1}-2 \psi_{i, j}+\psi_{i, j-1}}{\delta y^{2}}=\frac{\tilde{u}_{i+\frac{1}{2}, j}-\tilde{u}_{i-\frac{1}{2}, j}}{\delta x}+\frac{\tilde{v}_{i, j+\frac{1}{2}}-\tilde{v}_{i, j-\frac{1}{2}}}{\delta y} .
$$

A equação (2.48) gera um sistema linear para $\psi_{i, j}$ cuja matriz é simétrica e definida positiva. Para resolver esse sistema linear, empregamos o método dos gradientes conjugados.

A velocidade final é obtida discretizando as equações nos respectivos nós, ou seja

$$
\left\{\begin{array}{l}
u_{i+\frac{1}{2} j}^{n+1}=\tilde{u}_{i+\frac{1}{2} j}-\frac{\psi_{i+1 j}-\psi_{i j}}{\delta x}, \\
v_{i j+\frac{1}{2}}^{n+1}=\tilde{v}_{i j+\frac{1}{2}}-\frac{\psi_{i j+1}-\psi_{i j}}{\delta y} .
\end{array} .\right.
$$

A pressão é calculada aplicando a equação (2.43) no centro da célula,

$$
p_{i j}=\tilde{p}_{i j}+\frac{\psi_{i j}}{\delta t}
$$

As componentes do tensor não-Newtoniano, conforme equações (2.10)-(2.12), são aproximadas pela técnica de diferenças finitas como segue: a derivada temporal é aproximada explicitamente pelo método de Euler, os temos convectivos são calculados através do método 'upwind' de alta ordem CUBISTA e as derivadas espaciais de primeira ordem são aproximadas 
por diferenças centrais. Assim, temos:

$$
\begin{aligned}
& S_{i j}^{x y(n+1)}=S_{i j}^{x y}+\delta t\left\{-f\left(S_{k k}\right)_{i, j} \frac{1}{W e} S_{i, j}^{x y}-\operatorname{conv}\left(u S^{x y}\right)_{i, j}-\operatorname{conv}\left(v S^{x y}\right)_{i, j}\right. \\
& -\left[\left(\frac{\xi}{2}-1\right) \frac{v_{i+\frac{1}{2}, j}-v_{i-\frac{1}{2}, j}}{\delta x}+\frac{\xi}{2} \frac{u_{i, j+\frac{1}{2}}-u_{i, j-\frac{1}{2}}}{\delta y}\right] S_{i j}^{x x} \\
& -\left[\left(\frac{\xi}{2}-1\right) \frac{u_{i, j+\frac{1}{2}}-u_{i, j-\frac{1}{2}}}{\delta y}+\frac{\xi}{2} \frac{v_{i+\frac{1}{2}, j}-v_{i-\frac{1}{2}, j}}{\delta x}\right] S_{i, j}^{y y}+\left[1-f\left(S_{k k}\right)_{i, j}\right] \frac{2}{R e W e} D_{i, j}^{x y} \\
& -\frac{2}{R e}\left\{\frac{D_{i, j}^{x y(n+1)}-D_{i, j}^{x y}}{\delta t}+\operatorname{conv}\left(u D^{x y}\right)_{i, j}+\operatorname{conv}\left(v D^{x y}\right)_{i, j}\right. \\
& +\left[\left(\frac{\xi}{2}-1\right) \frac{v_{i+\frac{1}{2}, j}-v_{i-\frac{1}{2}, j}}{\delta x}+\frac{\xi}{2} \frac{u_{i, j+\frac{1}{2}}-u_{i, j-\frac{1}{2}}}{\delta y}\right] D_{i, j}^{x x} \\
& \left.\left.+\left[\left(\frac{\xi}{2}-1\right) \frac{u_{i, j+\frac{1}{2}}-u_{i, j-\frac{1}{2}}}{\delta y}+\frac{\xi}{2} \frac{v_{i+\frac{1}{2}, j}-v_{i-\frac{1}{2}, j}}{\delta x}\right] D_{i, j}^{y y}\right\}\right\}, \\
& S_{i j}^{x x(n+1)}=S_{i j}^{x x}+\delta t\left\{-f\left(S_{k k}\right)_{i, j} \frac{1}{W e} S_{i, j}^{x x}-\operatorname{conv}\left(u s^{x y}\right)_{i, j}-\operatorname{conv}\left(v S^{x y}\right)_{i, j}\right. \\
& -\left[(\xi-2) \frac{u_{i, j+\frac{1}{2}}-u_{i, j-\frac{1}{2}}}{\delta y}+\xi \frac{v_{i+\frac{1}{2}, j}-v_{i-\frac{1}{2}, j}}{\delta x}\right] S_{i, j}^{x y} \\
& -2(\xi-1) \frac{u_{i+\frac{1}{2}, j}-u_{i-\frac{1}{2}, j}}{\delta x} S_{i, j}^{x x}+\left[1-f\left(S_{k k}\right)_{i, j}\right] \frac{2}{R e W e} D_{i, j}^{x x} \\
& -\frac{2}{R e}\left\{\frac{D_{i, j}^{x x(n+1)}-D_{i, j}^{x x}}{\delta t}+\operatorname{conv}\left(u D^{x x}\right)_{i, j}+\operatorname{conv}\left(v D^{x x}\right)_{i, j}+2(\xi-1)\left(D_{i, j}^{x x}\right)^{2}\right. \\
& \left.\left.+\left[(\xi-2) \frac{u_{i, j+\frac{1}{2}}-u_{i, j-\frac{1}{2}}}{\delta y}+\xi \frac{v_{i+\frac{1}{2}, j}-v_{i-\frac{1}{2}, j}}{\delta x}\right] D^{x y}\right\}\right\}, \\
& S_{i j}^{y y(n+1)}=S_{i j}^{y y}+\delta t\left\{-f\left(S_{k k}\right)_{i j} \frac{1}{W e} S_{i j}^{y y}-\operatorname{conv}\left(u S^{x x}\right)_{i, j}-\operatorname{conv}\left(v S^{x x}\right)_{i, j}\right. \\
& -\left[(\xi-2) \frac{v_{i+\frac{1}{2}, j}-v_{i-\frac{1}{2}, j}}{\delta x}+\xi \frac{u_{i, j+\frac{1}{2}}-u_{i, j-\frac{1}{2}}}{\delta y}\right] S_{i, j}^{x y} \\
& -2(\xi-1) \frac{v_{i, j+\frac{1}{2}}-v_{i, j-\frac{1}{2}}}{\delta y} S_{i, j}^{y y}+\left[1-f\left(S_{k k}\right)_{i, j}\right] \frac{2}{R e W e} D_{i, j}^{y y} \\
& -\frac{2}{R e}\left\{\frac{D_{i, j}^{y y(n+1)}-D_{i, j}^{y y}}{\delta t}+\operatorname{conv}\left(u D^{y y}\right)_{i, j}+\operatorname{conv}\left(v D^{y y}\right)_{i, j}+2(\xi-1)\right)\left(D_{i, j}^{y y}\right)^{2} \\
& \left.\left.+\left[(\xi-2) \frac{v_{i+\frac{1}{2}, j}-v_{i-\frac{1}{2}, j}}{\delta x}+\xi \frac{u_{i, j+\frac{1}{2}}-u_{i, j-\frac{1}{2}}}{\delta y}\right] D_{i, j}^{x y}\right\}\right\},
\end{aligned}
$$

onde

$$
D_{i, j}^{x y}=\frac{1}{2}\left(\frac{u_{i, j+\frac{1}{2}}-u_{i, j-\frac{1}{2}}}{\delta y}+\frac{v_{i+\frac{1}{2}, j}-v_{i-\frac{1}{2}, j}}{\delta x}\right), D_{i, j}^{x x}=\left(\frac{u_{i+\frac{1}{2}, j}-u_{i-\frac{1}{2}, j}}{\delta x}\right)
$$




$$
D_{i, j}^{y y}=\left(\frac{v_{i, j+\frac{1}{2}}-v_{i, j-\frac{1}{2}}}{\delta x}\right) \quad \text { and } \quad f\left(S_{k k}\right)_{i, j}=1+\varepsilon \operatorname{ReWe}\left(S_{i, j}^{x x}+S_{i, j}^{y y}\right) .
$$

Os termos $\operatorname{conv}\left(u S^{x y}\right)_{i, j}, \cdots, \operatorname{conv}\left(u S^{y y}\right)_{i, j}$ representam os termos convectivos das equações (2.10)-(2.12) e são aproximados pelo método CUBISTA.

Nas equações (2.49)-(2.51), os termos que não estão na posição definida são obtidos fazendo-se a seguinte média

$$
u_{i, j+\frac{1}{2}}:=\frac{u_{i+\frac{1}{2}, j}+u_{i+\frac{1}{2}, j+1}+u_{i-\frac{1}{2}, j}+u_{i-\frac{1}{2}, j+1}}{4}, \quad v_{i+\frac{1}{2}, j}:=\frac{v_{i, j+\frac{1}{2}}+v_{i+1, j+\frac{1}{2}}+v_{i, j-\frac{1}{2}}+v_{i+1, j-\frac{1}{2}}}{4} .
$$

\subsubsection{Aproximação das equações nos contornos}

Vimos anteriormente que nos contornos rígidos adota-se a condição não-escorregamento para as componentes de velocidade, ou seja, $u=0$ e $v=0$. Nos contornos de injeção assume-se que $u_{n}=U, u_{m}=0, S^{x y}=0, S^{x x}=0$ e $S^{y y}=0$ e em contornos de ejeção assume-se a condição de Neumann homogênea, isto é,

$$
\frac{\partial u_{n}}{\partial n}=\frac{\partial u_{m}}{\partial n}=0, \quad \frac{\partial S^{x x}}{\partial n}=\frac{\partial S^{x y}}{\partial n}=\frac{\partial S^{y y}}{\partial n}=0 .
$$

Os subscritos $n$ e $m$ denotam as direções normais e tangenciais aos contornos.

\section{Aproximação das equações no contorno de entrada de fluido}

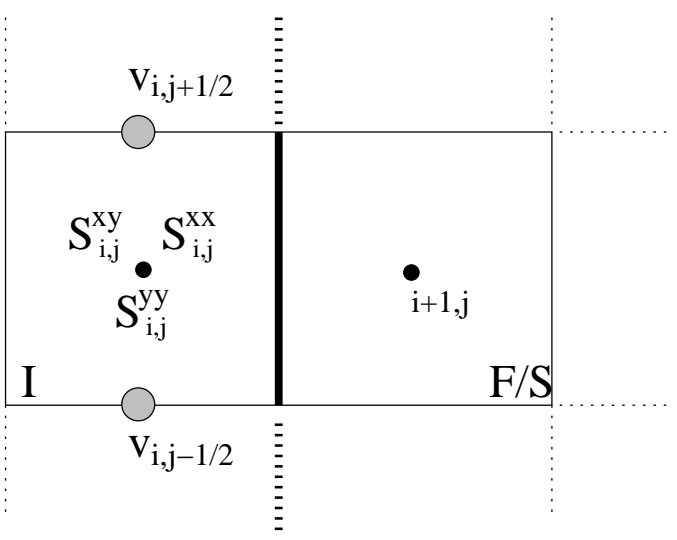

Figura 2.8: Célula do "inflow"com a face direita em contato com célula do interior do domínio.

Assim como no caso do contorno rígido, temos também quatro possíveis configurações de células do contorno de entrada de fluido. Para ilustrar a discretização das variáveis neste tipo de contorno adotaremos a configuração mostrada na figura 2.8 .

Adotando as condições de contorno apresentadas nas Seções anteriores, a discretização das componentes da velocidade e do tensor não-Newtoniano são dadas por

$$
u_{i+\frac{1}{2}, j}=U, \quad v_{i, j+\frac{1}{2}}=-v_{i+1, j+\frac{1}{2}} \cdot
$$




$$
S_{i, j}^{x x}=-S_{i+1, j}^{x x}, \quad S_{i, j}^{x y}=-S_{i+1, j}^{x y} \quad \text { and } \quad S_{i, j}^{y y}=-S_{i+1, j}^{y y} .
$$

Para as outras configurações de células do contorno de entrada de fluido as aproximações são obtidas de maneira análoga.

\section{Aproximação das equações no contorno de saída de fluido}

Neste tipo de contorno, para ilustrar a discretização das variávies adotaremos a configuração de célula mostrada na figura 2.9 .

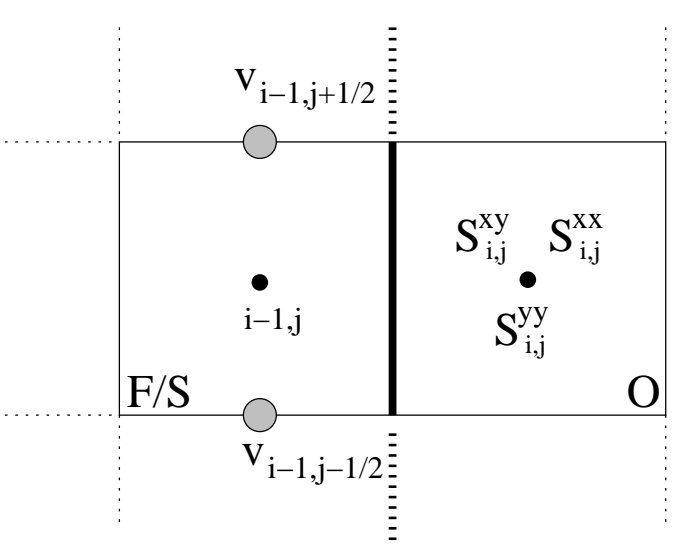

Figura 2.9: Célula do "outflow"com a face direita em contato com célula do interior do domínio.

Assim, adotando as condições de contorno apresentadas nas Seções anteriores, a discretização das componentes da velocidade e do tensor não-Newtoniano são dadas por

$$
\begin{gathered}
u_{i-\frac{1}{2}, j}=u_{i-\frac{3}{2}, j}, \quad v_{i, j+\frac{1}{2}}=v_{i-1, j+\frac{1}{2}} \cdot \\
S_{i, j}^{x x}=S_{i-1, j}^{x x}, \quad S_{i, j}^{x y}=S_{i-1, j}^{x y} \quad \text { and } \quad S_{i, j}^{y y}=S_{i-1, j}^{y y} .
\end{gathered}
$$

Para as outras configurações de células do contorno de entrada de fluido as aproximações são obtidas de maneira análoga.

\section{Aproximação das equações no contorno rígido}

A figura 2.10 apresenta as quatro possiveis configurações de células do contorno rígido. Com relação a figura 2.10(a), a condição não-escorregamento aplicada nas componentes da velocidade resulta nas equações,

$$
u_{i+\frac{1}{2}, j}=-u_{i+\frac{1}{2}, j-1} \quad \text { e } \quad v_{i, j-\frac{1}{2}}=0 .
$$

Para as outras configurações de células de contorno, o cálculo da velocidade de acordo com a condição não-escorregamento, segue de maneira análoga. O cálculo do tensor não-Newtoniano em contornos rígidos será apresentado na seção 2.7.4. 


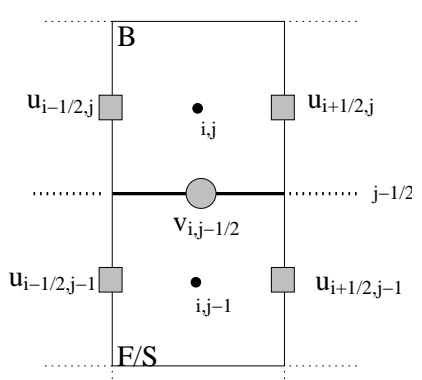

(a)

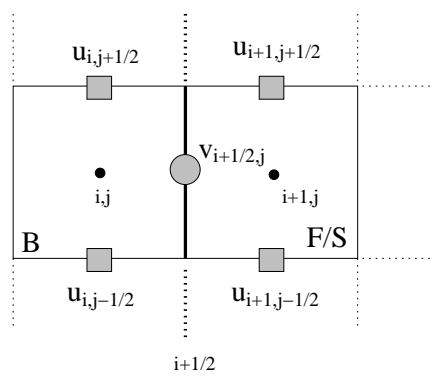

(c)

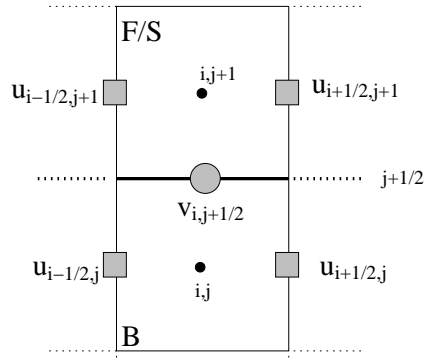

(b)

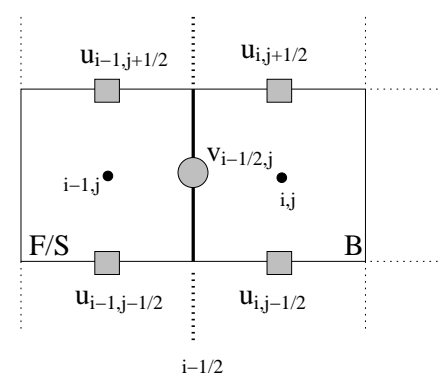

(d)

Figura 2.10: Configurações das células do contorno rígido (B).

\subsubsection{Aproximação do tensor não-Newtoniano em contornos rígidos}

Neste trabalho, os termos convectivos presentes nas equações das componentes do tensor não-Newtoniano (2.49)-(2.51) são aproximados pelo método 'upwind' de alta ordem CUBISTA. A equações do tensor não-Newtoniano são discretizadas no centro da face da célula do contorno rígido (B) a qual está em contato com uma célula do interior do domínio. Os valores de $S^{x y}$, $S^{x x}$ e $S^{y y}$ nas células do contorno são obtidos através das equações derivadas na Seção 2.5. A discretização em contornos paralelos ao eixo- $x$ e paralelos ao eixo- $y$ são considerados a seguir.

\section{- Contornos rígidos paralelos ao eixo- $x$}

Nestes contornos há dois casos a serem considerados, as células de contorno rígido (B) com apenas a face superior ou inferior em contato com uma célula do interior do domínio. Para ilustrar a discretização das equações derivadas na Seção 2.5 nesses contornos adota-se o caso representado na figura 2.11 .

Considerando a configuração de célula mostrada na figura 2.11 e as equações (2.33)-(2.35), que foram derivadas especialmente para esses tipos de contornos, os valores das componentes do tensor não-Newtoniano são dados por 


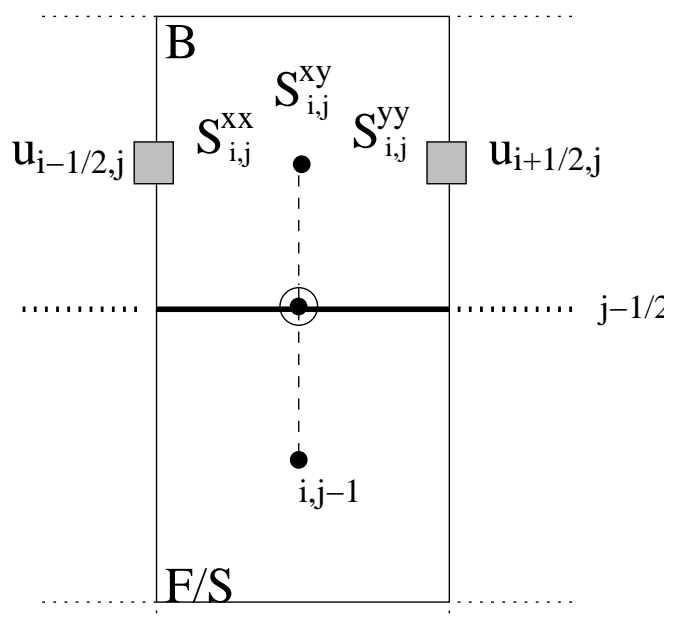

Figura 2.11: Célula do contorno rígido com a face inferior em contato com uma célula do interior do domínio.

$$
\begin{aligned}
& S_{i, j-\frac{1}{2}}^{x y(n+1)}=\frac{1}{\left.D e n S^{x y}\right|_{i, j-\frac{1}{2}}}\left\{e^{-\frac{1}{W e} \delta t} S_{i, j-\frac{1}{2}}^{x y}-\left.e^{-\frac{1}{W e} \delta t} \frac{\delta t}{2}\left[\left(\frac{\xi}{2}+\varepsilon\right) S_{i, j-\frac{1}{2}}^{x x}+\left(\frac{\xi}{2}+\varepsilon-1\right) S_{i, j-\frac{1}{2}}^{y y}\right] \frac{\partial u}{\partial y}\right|_{i, j-\frac{1}{2}}\right. \\
& \left.-\frac{1}{R e}\left[\left.\frac{\partial u^{(n+1)}}{\partial y}\right|_{i, j-\frac{1}{2}}-\left.\left(1-e^{-\frac{1}{W e} \delta t}\right) \frac{\left.\partial u^{(*)}\right)}{\partial y}\right|_{i, j-\frac{1}{2}}-\left.e^{-\frac{1}{W e} \delta t} \frac{\partial u}{\partial y}\right|_{i, j-\frac{1}{2}}\right]\right\} \\
& S_{i, j-\frac{1}{2}}^{x x(n+1)}=e^{-\frac{1}{W e} \delta t} S_{i, j-\frac{1}{2}}^{x x} \quad-(\xi-2) \frac{\delta t}{2}\left(\left.\frac{\partial u}{\partial y}\right|_{i, j-\frac{1}{2}} e^{-\frac{1}{W e} \delta t} S_{i, j-\frac{1}{2}}^{x y}+\left.\frac{\partial u^{(n+1)}}{\partial y}\right|_{i, j-\frac{1}{2}} S_{i, j-\frac{1}{2}}^{x y(n+1)}\right) \\
& -(\xi-2) \frac{W e}{R e}\left(1-e^{-\frac{1}{W e} \delta t}\right)\left(\left.\frac{\partial u^{(*)}}{\partial y}\right|_{i, j-\frac{1}{2}}\right)^{2} \\
& S_{i, j-\frac{1}{2}}^{y y(n+1)}=e^{-\frac{1}{W e} \delta t} S_{i, j-\frac{1}{2}}^{y y}-\xi \frac{\delta t}{2}\left(\left.\frac{\partial u}{\partial y}\right|_{i, j-\frac{1}{2}} e^{-\frac{1}{W e} \delta t} S_{i, j-\frac{1}{2}}^{x y}+\left.\frac{\partial u^{(n+1)}}{\partial y}\right|_{i, j-\frac{1}{2}} S_{i, j-\frac{1}{2}}^{x y(n+1)}\right) \\
& -\xi \frac{W e}{R e}\left(1-e^{-\frac{1}{W e} \delta t}\right)\left(\left.\frac{\partial u^{(*)}}{\partial y}\right|_{i, j-\frac{1}{2}}\right)^{2}
\end{aligned}
$$

onde o valor de $\left.\frac{\partial u^{(*)}}{\partial y}\right|_{i, j-\frac{1}{2}}$ é obtido pela média entre $\frac{\partial u}{\partial y}\left(x_{i}, y_{j-\frac{1}{2}}, t\right)$ nos tempos $t_{n}$ e $t_{n+1}$, ou 
seja,

$$
\left.\frac{\partial u\left(t^{*}\right)}{\partial y}\right|_{i, j-\frac{1}{2}}=\frac{1}{2}\left[\left.\frac{\partial u}{\partial y}\right|_{i, j-\frac{1}{2}}+\left.\frac{\partial u^{(n+1)}}{\partial y}\right|_{i, j-\frac{1}{2}}\right],
$$

e essas derivadas são aproximadas como segue,

$$
\left.\frac{\partial u}{\partial y}\right|_{i, j-\frac{1}{2}}=\frac{u_{i, j}^{n}-u_{i, j-1}^{n}}{\delta y},\left.\quad \frac{\partial u^{(n+1)}}{\partial y}\right|_{i, j-\frac{1}{2}}=\frac{u_{i, j}^{(n+1)}-u_{i, j-1}^{(n+1)}}{\delta y},
$$

e $\left.\operatorname{Den} S^{x y}\right|_{i, j-\frac{1}{2}}=1-\left[\xi^{2}-2 \xi+2(\xi-1) \varepsilon\right] \frac{\delta t^{2}}{4}\left(\left.\frac{\partial u^{(n+1)}}{\partial y}\right|_{i, j-\frac{1}{2}}\right)^{2}$

As velocidades nas posições $(i, j)$ e $(i, j-1)$ são dadas por

$$
u_{i, j}=\frac{u_{i+\frac{1}{2}, j}+u_{i-\frac{1}{2}, j}}{2} \text { e } \quad u_{i, j-1}=\frac{u_{i+\frac{1}{2}, j-1}+u_{i-\frac{1}{2}, j-1}}{2} \text {, respectivamente. }
$$

Finalmente, os valores de $S_{i, j}^{x y}, S_{i, j}^{x x}$ e $S_{i, j}^{y y}$ são obtidos pela interpolação linear usando os nós $\left(i, j-\frac{1}{2}\right)$ e $(i, j-1)$, respectivamente, como vê-se na figura 2.11 .

\section{- Contornos rígidos paralelos ao eixo-y}

Neste caso, (ver figura 2.12) assume-se que as células de contorno rígido possuem apenas a face direita (ou esquerda) em contato com uma célula do interior do domínio. Para ilustrar a discretização das equações derivadas na Seção 2.5 para esses contornos, considera-se o caso representado na figura 2.12 .

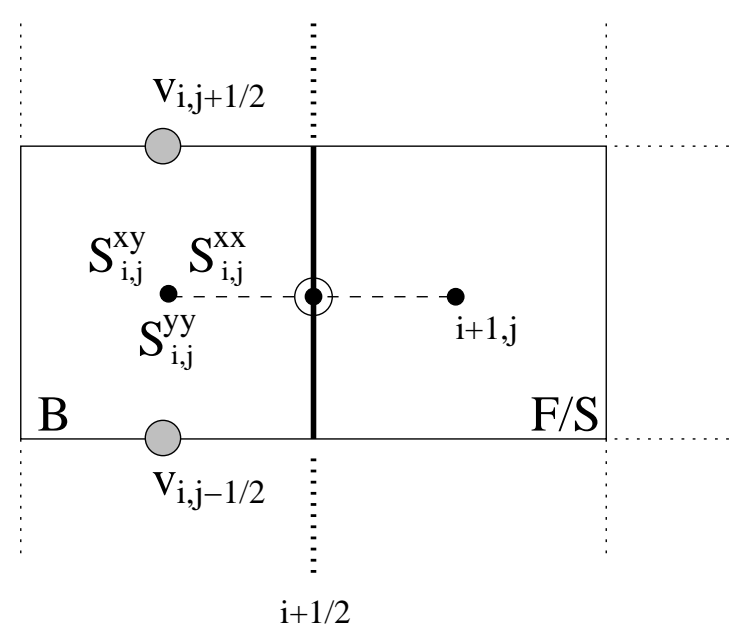

Figura 2.12: Célula do contorno rígido com a face direita em contato com célula do interior do domínio.

Considerando a figura 2.12, os valores das componentes do tensor não-Newtoniano são obtidos através das equações (2.37)-(2.39) aplicadas nos nós $\left(i+\frac{1}{2}, j\right)$, as quais fornecem as seguintes equações 


$$
\begin{aligned}
& S_{i+\frac{1}{2}, j}^{x y(n+1)}=\frac{1}{\left.D e n S^{x y}\right|_{i+\frac{1}{2}, j}}\left\{e^{-\frac{1}{W e} \delta t} S_{i+\frac{1}{2}, j}^{x y}-\left.e^{-\frac{1}{W e} \delta t} \frac{\delta t}{2}\left[\left(\frac{\xi}{2}+\varepsilon-1\right) S_{i+\frac{1}{2}, j}^{x x}+\left(\frac{\xi}{2}+\varepsilon\right) S_{i+\frac{1}{2}, j}^{y y}\right] \frac{\partial v}{\partial x}\right|_{i+\frac{1}{2}, j}\right. \\
& \left.-\frac{1}{R e}\left[\left.\frac{\partial v^{(n+1)}}{\partial x}\right|_{i+\frac{1}{2}, j}-\left.\left(1-e^{-\frac{1}{W e} \delta t}\right) \frac{\partial v^{(*)}}{\partial x}\right|_{i+\frac{1}{2}, j}-\left.e^{-\frac{1}{W e} \delta t} \frac{\partial v}{\partial x}\right|_{i+\frac{1}{2}, j}\right]\right\} \\
& S_{i+\frac{1}{2}, j}^{x x(n+1)}=e^{-\frac{1}{W e} \delta t} S_{i+\frac{1}{2}, j}^{x x}-\xi \frac{\delta t}{2}\left(\left.\frac{\partial v}{\partial x}\right|_{i+\frac{1}{2}, j} e^{-\frac{1}{W e} \delta t} S_{i+\frac{1}{2}, j}^{x y}+\left.\frac{\partial v^{(n+1)}}{\partial x}\right|_{i+\frac{1}{2}, j} \begin{array}{r}
S_{i+\frac{1}{2}, j}^{x y}(n+1) \\
i+1
\end{array}\right) \\
& -\xi \frac{W e}{R e}\left(1-e^{-\frac{1}{W e} \delta t}\right)\left(\left.\frac{\partial v^{(*)}}{\partial x}\right|_{i+\frac{1}{2}, j}\right)^{2} \\
& \underset{i+\frac{1}{2}, j}{S^{y y}(n+1)}=e^{-\frac{1}{W e} \delta t} S_{i+\frac{1}{2}, j}^{y y}-(\xi-2) \frac{\delta t}{2}\left(\left.\frac{\partial v}{\partial x}\right|_{i+\frac{1}{2}, j} e^{-\frac{1}{W e} \delta t} S_{i+\frac{1}{2}, j}^{x y}+\left.\frac{\partial v^{(n+1)}}{\partial x}\right|_{i+\frac{1}{2}, j} \begin{array}{r}
S_{i+\frac{1}{2}, j}^{x y}(n+1) \\
i
\end{array}\right) \\
& -(\xi-2) \frac{W e}{R e}\left(1-e^{-\frac{1}{W e} \delta t}\right)\left(\left.\frac{\partial v^{(*)}}{\partial x}\right|_{i+\frac{1}{2}, j}\right)^{2}
\end{aligned}
$$

onde o valor de $\left.\frac{\partial v^{(*)}}{\partial x}\right|_{i+\frac{1}{2}, j}$ é obtido pela média entre $\frac{\partial v}{\partial x}\left(x_{i+\frac{1}{2}}, y_{j}, t\right)$ nos tempos $t_{n}$ e $t_{n+1}$, ou seja,

$$
\left.\frac{\partial v^{(*)}}{\partial x}\right|_{i+\frac{1}{2}, j}=\frac{1}{2}\left[\left.\frac{\partial v}{\partial x}\right|_{i+\frac{1}{2}, j}+\left.\frac{\partial v^{(n+1)}}{\partial x}\right|_{i+\frac{1}{2}, j}\right]
$$

e essas derivadas são aproximadas como segue,

$$
\left.\frac{\partial v}{\partial x}\right|_{i+\frac{1}{2}, j}=\frac{v_{i+1, j}^{n}-v_{i, j}^{n}}{\delta x},\left.\quad \frac{\partial v^{(n+1)}}{\partial x}\right|_{i+\frac{1}{2}, j}=\frac{v_{i+1, j}^{n+1}-v_{i, j}^{n+1}}{\delta x}
$$

e $\left.\operatorname{Den} S^{x y}\right|_{i+\frac{1}{2}, j}=1-\left[\xi^{2}-2 \xi+2(\xi-1) \varepsilon\right] \frac{\delta t^{2}}{4}\left(\left.\frac{\partial v\left(t_{n+1}\right)}{\partial x}\right|_{i+\frac{1}{2}, j}\right)^{2}$

As velocidades nas posições $(i+1, j)$ e $(i, j)$ são dadas por

$$
v_{i+1, j}=\frac{v_{i+1, j+\frac{1}{2}}+v_{i+1, j-\frac{1}{2}}}{2} \quad \text { e } \quad v_{i, j}=\frac{v_{i, j+\frac{1}{2}}+v_{i, j-\frac{1}{2}}}{2} \text {, respectivamente. }
$$

Finalmente, os valores de $S_{i, j}^{x y}, S_{i, j}^{x x}$ e $S_{i, j}^{y y}$ são obtidos pela interpolação linear usando os nós $\left(i+\frac{1}{2}, j\right)$ e $(i+1, j)$, respectivamente, como vê-se na figura 2.12. 


\subsubsection{Aproximação das equações na superfície livre}

As equações (2.14)-(2.15) representam as condições apropriadas para a velocidade e pressão na superfície livre. Para aplicar estas condições procedemos como segue.

Assume-se uma malha uniforme $\delta x=\delta y$ e que o espaçamento na malha é suficientemente pequeno de forma que a superfície livre intercepta a célula apenas em duas faces. Dessa maneira, as condições (2.14) e (2.15) podem ser aplicadas de acordo com os três casos seguintes.

\section{- Células de superfície com apenas uma face em contato com células vazias}

Nessas células considera-se que a superfície é horizontal ou vertical de acordo com qual das faces está em contato com uma célula vazia (E). Neste caso tem-se que o vetor normal a superfície é da seguinte forma $\mathbf{n}=\left(n_{x}, 0\right)$ ou $\mathbf{n}=\left(0, n_{y}\right)$. Considerando o caso mostrado na

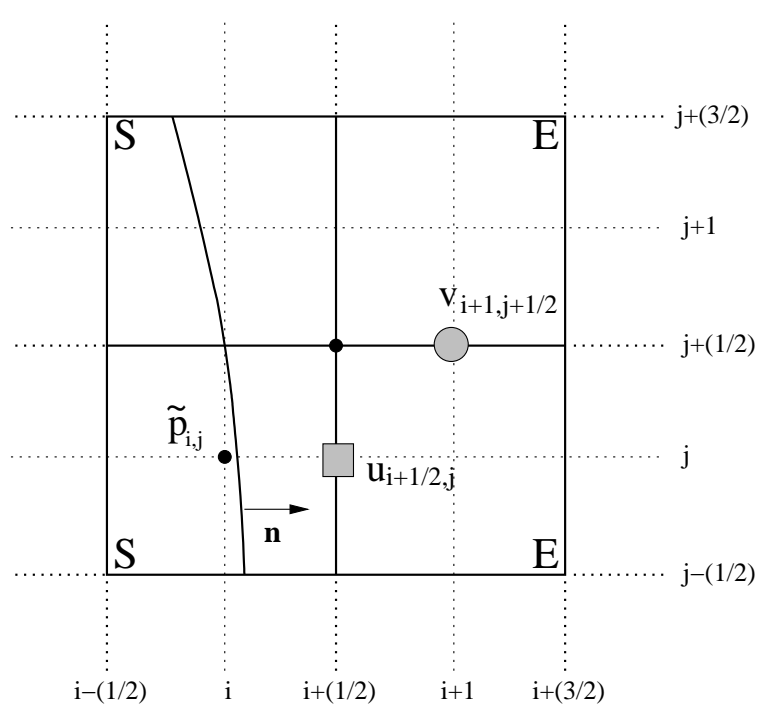

Figura 2.13: Células de superfície com apenas uma face em contato com células vazias.

figura 2.13 tem-se $\mathbf{n}=(1,0)$. Neste caso as condições $(2.14)$ e (2.15) reduzem-se a

$$
\begin{gathered}
\tilde{p}=2 \frac{1}{R e} \frac{\partial u}{\partial x}+S^{x x} \\
\frac{1}{R e}\left(\frac{\partial u}{\partial y}+\frac{\partial v}{\partial x}\right)+S^{x y}=0 .
\end{gathered}
$$

Quando a velocidade é calculada pelas equações (2.45) e (2.46), os valores de $\tilde{p}_{i, j}, u_{i+\frac{1}{2}, j}, v_{i+1, j+\frac{1}{2}}$ são requeridos. Estes valores podem ser obtidos como segue.

Discretizando a equação da continuidade no centro da célula obtém-se

$$
u_{i+\frac{1}{2}, j}=u_{i-\frac{1}{2}, j}-\frac{\delta x}{\delta y}\left(v_{i, j+\frac{1}{2}}-v_{i, j-\frac{1}{2}}\right) .
$$


Agora, aproximando a condição (2.59) na posição $\left(i+\frac{1}{2}, j+\frac{1}{2}\right)$ vem,

$$
v_{i+1, j+\frac{1}{2}}=v_{i, j+\frac{1}{2}}-\frac{\delta x}{\delta y} \frac{\left(u_{i+\frac{1}{2}, j+1}-u_{i+\frac{1}{2}, j}\right)}{\delta y}-\delta x \operatorname{Re} S_{i+\frac{1}{2}, j+\frac{1}{2}}^{x y} .
$$

O valor de $S_{i+\frac{1}{2}, j+\frac{1}{2}}^{x y}$ é obtido fazendo uma interpolação usando $S_{i, j+\frac{1}{2}}^{x y}=0.5\left(S_{i, j}^{x y}+S_{i, j+1}^{x y}\right)$ e $S_{i-1, j+\frac{1}{2}}^{x y}=0.5\left(S_{i-1, j}^{x y}+S_{i-1, j+1}^{x y}\right)$. Assim, tendo calculado as velocidades por meio das equações (2.60) e (2.61), a pressão $\tilde{p}_{i, j}$ segue da equação (2.58) aplicando-a no centro da célula. Logo, tem-se

$$
\tilde{p}_{i, j}=\frac{2}{R e} \frac{u_{i+\frac{1}{2}, j}-u_{i-\frac{1}{2}, j}}{\delta x}+S_{i, j}^{x x} .
$$

Há três outras configurações possíveis de células de superfície com apenas uma face em contato com uma célula vazia. As velocidades e pressão para essas células são calculadas de maneira semelhante.

\section{Células de superfície com duas faces adjacentes em contato com células vazias}

Para estes tipos de células assume-se que o vetor normal faz um ângulo de $45^{\circ}$ entre as duas faces adjacentes em contato com as células vazias e considera-se o vetor normal como sendo $\mathbf{n}=\left( \pm \frac{\sqrt{2}}{2}, \pm \frac{\sqrt{2}}{2}\right)$. Neste caso, as condições (2.14) e (2.15) reduzem-se a

$$
\begin{gathered}
\tilde{p}= \pm\left[\frac{1}{R e}\left(\frac{\partial u}{\partial y}+\frac{\partial v}{\partial x}\right)+\frac{1}{2}\left(S^{x x}+S^{y y}\right)+S^{x y}\right] \\
\frac{1}{R e}\left(\frac{\partial u}{\partial x}-\frac{\partial v}{\partial y}\right)+\frac{1}{2}\left(S^{x x}-S^{y y}\right)=0
\end{gathered}
$$

respectivamente.

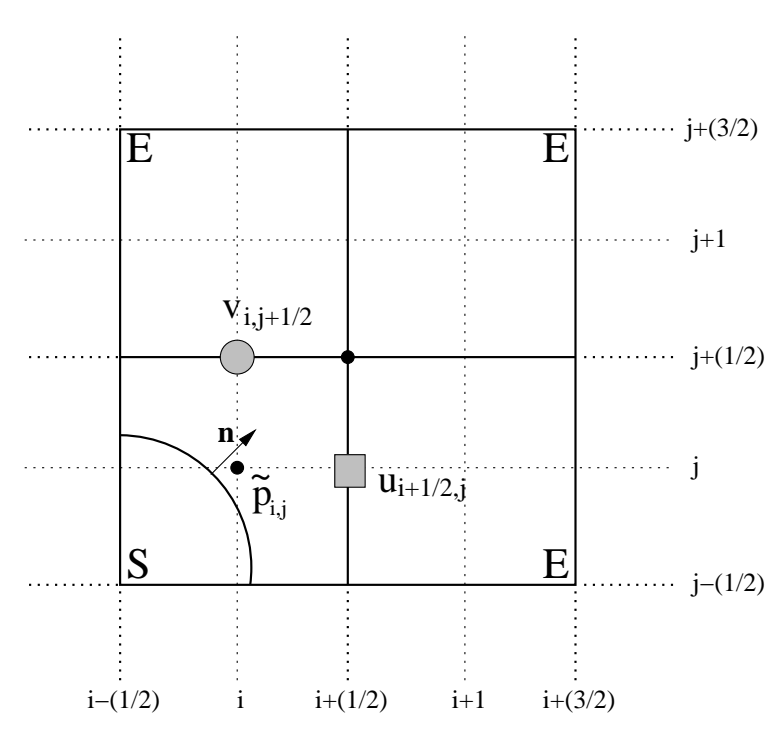

Figura 2.14: Célula de superfície com duas faces adjacentes em contato com células vazias. 
Considerando a figura 2.14, observa-se que os valores $\tilde{p}_{i, j}, u_{i+\frac{1}{2}, j}$ e $v_{i, j \frac{1}{2}}$ são necessários quando calcula-se as velocidade intermediárias e o tensor não-Newtoniano. Esses valores são obtidos como segue. As velocidades $u_{i+\frac{1}{2}, j}$ e $v_{i, j+\frac{1}{2}}$ são obtidas discretizando a equação da continuidade e a condição (2.63) são no centro da célula resultando em

$$
\left\{\begin{array}{l}
\frac{u_{i+\frac{1}{2}, j}-u_{i-\frac{1}{2}, j}}{\delta x}+\frac{v_{i, j+\frac{1}{2}}-v_{i, j-\frac{1}{2}}}{\delta y}=0 \\
\frac{1}{\delta x}\left(u_{i, j}-u_{i-1, j}\right)-\frac{1}{\delta y}\left(v_{i, j}-v_{i, j-1}\right)=-\frac{R e}{2}\left(S_{i, j}^{x x}-S_{i, j}^{y y}\right)
\end{array}\right.
$$

O sistema $2 \times 2(2.64)$ é resolvido para as velocidades $u_{i+\frac{1}{2}, j}$ e $v_{i, j+\frac{1}{2}}$ e obtém-se

$$
\begin{aligned}
& u_{i+\frac{1}{2}, j}=u_{i-\frac{1}{2}, j}-\frac{\delta x R e}{4}\left(S_{i, j}^{x x}-S_{i, j}^{y y}\right), \\
& v_{i, j+\frac{1}{2}}=v_{i, j-\frac{1}{2}}+\frac{\delta y R e}{4}\left(S_{i, j}^{x x}-S_{i, j}^{y y}\right) .
\end{aligned}
$$

Sendo conhecidas as velocidades $u_{i+\frac{1}{2}, j}$ e $v_{i, j+\frac{1}{2}}$, a pressão é avaliada no centro da célula pela equação (2.62) produzindo,

$$
\tilde{p}_{i, j}=\frac{1}{R e}\left(\frac{u_{i, j+\frac{1}{2}}-u_{i, j-\frac{1}{2}}}{\delta y}+\frac{v_{i+\frac{1}{2}, j}-v_{i-\frac{1}{2}, j}}{\delta x}\right)+\frac{1}{2}\left(S_{i, j}^{x x}+S_{i, j}^{y y}\right)+S_{i, j}^{x y} .
$$

Existem outros três tipos de células de superfície com duas faces adjacentes em contato com células vazias. O cálculo das velocidades e da pressão para essas células é efetuado de maneira análoga.

\section{- Células de superfície com duas faces opostas em contato com células vazias}

Para essas células não obtém-se uma aproximação para o vetor normal. A velocidade é ajustada de forma qua a equação da continuidade seja satisfeita. A pressão e as componentes do tensor não-Newtoniano são definidos como nulos Se essas células aparecem durante a execução de um dado problema, a malha deverá ser refinada para minimizar o aparecimento das mesmas. 


\subsection{Verificação do método numérico}

As equações de diferenças finitas que descrevem o método numérico desenvolvido nesse trabalho, nomeado GENSMAC2D-PTT, foram incorporadas ao sistema Freeflow2D [52] para simular escoamentos governados pela equação constitutiva PTT.

Para validar GENSMAC2D-PTT, consideramos o escoamento totalmente desenvolvido em um canal bidimensional. Recentemente, Oliveira \& Pinho [54] e Alves et al. [2] encontraram a solução analítica deste problema para os modelos SPTT e PTT, respectivamente. Para obter a solução analítica para este problema foram impostas as hipóteses do escoamento totalmente desenvolvido em um canal bidimensional e o sistema de equações resultante foi resolvido analíticamente afim de obter expressões para as componentes da velocidade e do tensor não-Newtoniano. Ressaltamos que nos trabalhos [54] e [2], Oliveira \& Pinho e Alves et al. consideraram o problema e todas as equações envolvidas, na forma dimensional. Detalhes de como obter a solução analítica dos modelos SPTT e PTT para escoamentos totalmente desenvolvidos em um canal bidimensional são encontrados em [54] e [2].

Vale a pena ressaltar que o tensor não-Newtoniano $S_{i j}$ é uma variável auxiliar resultante da formulação do método numérico desenvolvido nesse trabalho. Logo, seguindo a notação deste trabalho, a variável presente na equação constitutiva do modelo PTT é o tensor extra-tensão $\tau_{i j}$ (ver equação (2.3)). Portanto, procuraremos sempre apresentar os resultados teóricos e numéricos em função do tensor $\tau_{i j}$.

\subsubsection{Solução analítica do modelo PTT em um escoamento total- mente desenvolvido em um canal bidimensional}

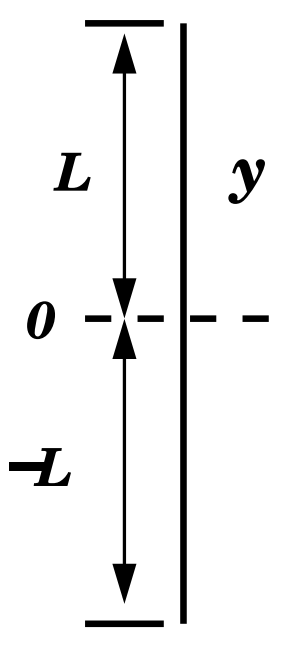

Figura 2.15: Esboço de um corte vertical em um canal bidimensional. Domínio de dependência das variáveis do problema.

Considere o escoamento totalmente desenvolvido em um canal bidimensional conforme 
mostrado na figura 2.15. Nesse caso, as seguintes hipóteses são válidas

$$
\frac{\partial}{\partial t} \equiv \frac{\partial}{\partial x} \equiv 0, \quad v=0, u=u(y) \text { e } \frac{\partial p}{\partial x}=\Delta \mathrm{p}_{\mathrm{x}}(\text { constante }) .
$$

O sistema de coordenadas considerado é centrado no eixo de simetria $y=0$ (ver figura 2.15). Assume-se que a velocidade $u(y)$ obedece a condição não-escorregamento em $y= \pm 1$, ou seja, $u(-1)=u(1)=0$ e que a solução $u(y)$ deve satisfazer $\frac{d u}{d y}(0)=0$. Neste caso, a equação de conservação de massa é satisfeita e as equações (2.2)-(2.3) reduzem-se a

$$
\left\{\begin{array}{l}
\frac{\partial \tau^{x y}}{\partial y}=\Delta \mathrm{p}_{\mathrm{x}} \\
\frac{\partial p}{\partial y}-\frac{\partial \tau^{y y}}{\partial y}=0 \\
\frac{1}{W e} f\left(\tau_{k k}\right) \tau^{x x}-(2-\xi) \frac{d u}{d y} \tau^{x y}-(2-\xi) \frac{1}{R e}\left(\frac{d u}{d y}\right)^{2}=0 \\
\frac{1}{W e} f\left(\tau_{k k}\right) \tau^{y y}+\xi \frac{d u}{d y} \tau^{x y}+\xi \frac{1}{R e}\left(\frac{d u}{d y}\right)^{2}=0 \\
\frac{1}{W e} f\left(\tau_{k k}\right) \tau^{x y}+\frac{\xi}{2} \frac{d u}{d y} \tau^{x x}+\left(\frac{\xi}{2}-1\right) \frac{d u}{d y} \tau^{y y}-\left[1-f\left(\tau_{k k}\right)\right] \frac{1}{R e W e} \frac{d u}{d y}=0
\end{array}\right.
$$

Ao resolver esse sistema, Alves et al. [2] obtiveram as seguintes expressões para a componente $u$ da velocidade e para as componentes $\tau^{x y}, \tau^{x x}$ e $\tau^{y y}$ do tensor extra-tensão, as quais escrevemos na forma adimensional:

$$
\begin{aligned}
& u(y)=\frac{R e}{\chi} \Delta \mathrm{p}_{\mathrm{x}}\left(1-y^{2}\right) \\
& +\frac{1}{2 R e W e^{2} \xi(2-\xi) \Delta \mathrm{p}_{\mathrm{x}}}\left(1+\frac{2}{\chi}\right)\left[\ln \frac{1+\sqrt{1-(a y)^{2}}}{1+\sqrt{1-a^{2}}}+\left(1+\sqrt{1-a^{2}}\right)-\left(1+\sqrt{1-(a y)^{2}}\right)\right] \\
& \tau^{x y}(y)=\Delta \mathrm{p}_{\mathrm{x}} y \\
& \tau^{x x}(y)=\frac{1}{2 \operatorname{ReWe\xi }}\left(1-\sqrt{1.0-(a y)^{2}}\right), a y \leq 1 \\
& \tau^{y y}(y)=-\frac{\xi}{(2-\xi)} \tau^{x x}(y)
\end{aligned}
$$


onde $\quad a=-2 R e W e \Delta \mathrm{p}_{\mathrm{x}} \sqrt{\xi(2-\xi)}, \xi \leq 2 \quad$ e $\quad \chi=\frac{\xi(2-\xi)}{\varepsilon(1-\xi)}$

O conjunto de equações (2.67)-(2.70) representa a solução exata, no estado estacionário, para o problema do escoamento totalmente desenvolvido em um canal bidimensional.

O tensor $\tau_{i j}$ e o tensor não-Newtoniano $S_{i j}$ estão relacionados pela formulação EVSS (ver equação (2.4)), a qual sob as hipóteses acima reduz-se a equação:

$$
\left(\begin{array}{ll}
\tau^{x x} & \tau^{x y} \\
\tau^{x y} & \tau^{y y}
\end{array}\right)=\frac{1}{R e}\left(\begin{array}{ll}
0 & \frac{d u}{d y} \\
\frac{d u}{d y} & 0
\end{array}\right)+\left(\begin{array}{ll}
S^{x x} & S^{x y} \\
S^{x y} & S^{y y}
\end{array}\right)
$$

Logo, podemos validar o método numérico desenvolvido comparando as soluções exatas (2.67)-(2.70) com as soluções numéricas obtidas com o GENSMAC2D-PTT. Observamos que a componente $\tau^{x x}$ do tensor extra-tensão não depende do parâmetro $\varepsilon$ presente no modelo PTT (ver equação (2.69)). Desta forma, uma outra verificação é variar o parâmetro $\varepsilon$ nas simulações e constatar que a solução numérica da componente $\tau^{x x}$ não varia com $\varepsilon$.

\subsubsection{Comparação da solução numérica com a solução analítica}

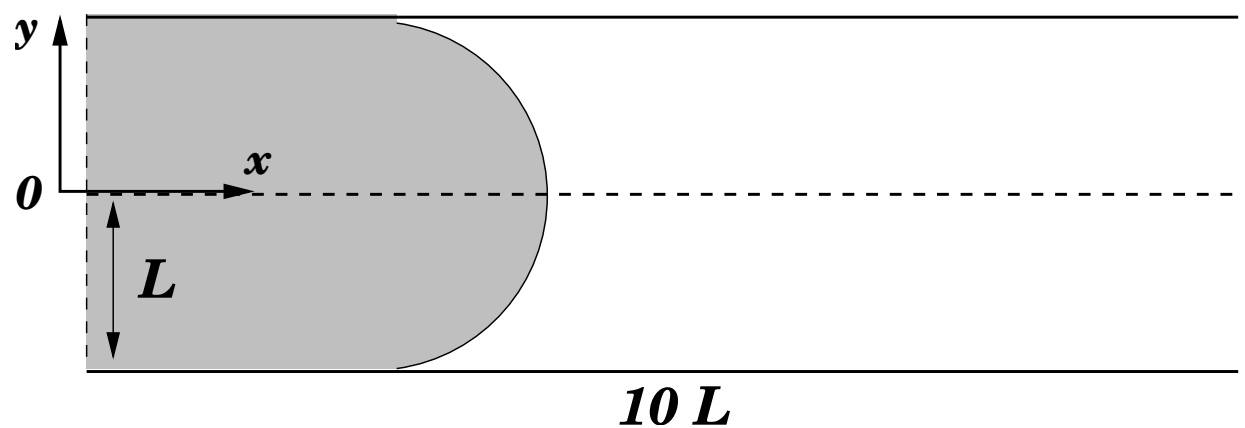

Figura 2.16: Definição do domínio para a simulação do escoamento em um canal bidimensional.

Para efeito de verificação do método GENSMAC2D-PTT, simulamos o problema do escoamento de fluidos viscoeláticos em um canal bidimensional como segue.

Considere o escoamento em um canal bidimensional formado por duas placas paralelas que estão a uma distância $L$ do eixo de simetria $y=0$ e possuem comprimento de $10 L$, como ilustrado na figure 2.16. No contorno de entrada de fluido impomos os valores exatos da velocidade $u(y)$ e das componentes do tensor extra-tensão $\tau^{x y}(y), \tau^{y y}(y)$ e $\tau^{x x}(y)$ dados pelas equações (2.67)-(2.70) enquanto que no contorno de saida e nas paredes do canal seguem as condições estabelecidas nas Seções anteriores.

A simulacao é iniciada com o canal vazio e o fluido é injetado na entrada do canal e o canal é então gradualmente preenchido com o fluido. Logo, inicialmente há uma superficie livre em movimento nessa simulação. Na superficie livre do fluido adota-se as condições para o tensor extra-tensão descritas nas Seções anteriores. 
Para simular esse problema. os seguintes dados que especificam o fluido foram considerados: $\varepsilon=0.5, \xi=0.01, \Delta \mathrm{p}_{\mathrm{x}}=-1.0, R e=1.0$ e $W e=1.25$

Com estes dados, os parâmetros de escalas empregados são:

$L=1.0 \mathrm{~m}$ (metade da largura do canal), $U=U \max =0.911874 \mathrm{~ms}^{-1}$ (calculado usando a equação (2.67)), $\nu=0.911874 \mathrm{~m}^{2} \mathrm{~s}^{-1}$ (valor usado para que $R e=1$ ) e $\lambda=1.370803 \mathrm{~s}$ (escolhido de forma que $W e=1.25)$.

Para analisar a convergência de GENSMAC2D-PTT simulamos esse problema utilizando três diferentes malhas:

Malha $1(\mathbf{M 1}): 10 \times 50$ células $(\delta x=\delta y=0.2)$,

Malha $2(\mathbf{M} 2): 20 \times 100$ células $(\delta x=\delta y=0.1)$,

Malha $3(\mathbf{M} 3): 40 \times 200$ células $(\delta x=\delta y=0.05)$.

Os resultados dessas simulações são mostrados e analisados a seguir.

No tempo $t=0$ o canal está vazio e um fluido com as caracteristicas citadas acima é injetado a partir do contorno de entrada de fluido até que o canal esteja completamente cheio e que o escoamento atinja o estado estacionário. A figura 2.17 mostra os perfis da componente $u$ da velocidade e das componentes do tensor extra-tensão no tempo $t=7 \mathrm{~s}$ e a figura 2.18 mostra estes perfis no tempo $t=100 \mathrm{~s}$. Esses resultados são provenientes das simulações utilizando a malha M3. Observe na figura 2.17 que o escoamento ainda está em desenvolvimento: as isolinhas não estão paralelas o que mostra o estado transiente do escoamento. Entretanto, em $t=100 \mathrm{~s}$, a figura 2.18 mostra o canal completamente cheio e as isolinhas das variáveis estão paralelas indicando o estado estacionário do escoamento.

Como o estado estádo estacionário foi atingido, a solução no interior do canal, mais especificamente em qualquer corte na vertical do canal, os valores numéricos devem ser concordar com os valores exatos que foram impostos no contorno de entrada do canal. Para verificar este fato, consideramos os valores numéricos das variáveis $u, \tau^{x y}, \tau^{x x}$ e $\tau^{x y}$ no meio do canal $(x=5)$ nas três malhas e comparamos com a solução analítica dada pelas equações (2.67)-(2.70). De fato, a figura 2.19 mostra a comparação da solução analítica com a solução numérica obtida no meio do canal utilizando as três malhas. Pode-se notar na figura 2.19 que as soluções numéricas obtidas nas três malhas possuem uma boa concordância com a solução analítica. Além disso, a figura 2.19 mostra que quando a malha é refinada a solução numérica converge para a solução analítica.

Para demonstrar a convergência de GENSMAC2D-PTT calculamos o erro relativo da solução numérica $(\mathrm{E}(\mathrm{SolNum}))$ na norma $\mathbf{L}_{2}$ em cada malha através da equação

$$
E(\operatorname{SolNum})=\sqrt{\sum_{j}\left(\operatorname{SolEx}\left(y_{j}\right)-\operatorname{SolNum}_{M_{i}}\left(y_{j}\right)\right)^{2}},
$$

os quais são mostrados na Tabela 2.1. Podemos observar na Tabela 2.1 que os erros decrescem quando a malha é refinada. Estes resultados demonstram que método numérico GENSMAC2D-PTT converge quando a malha é refinada. 


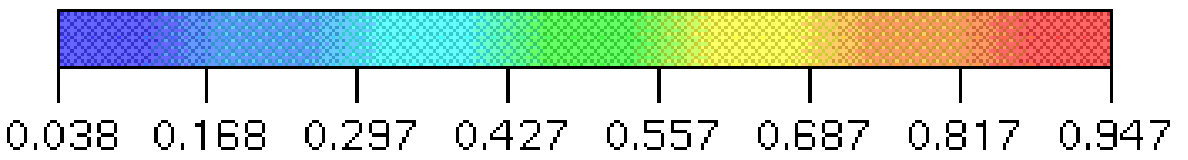

(a)
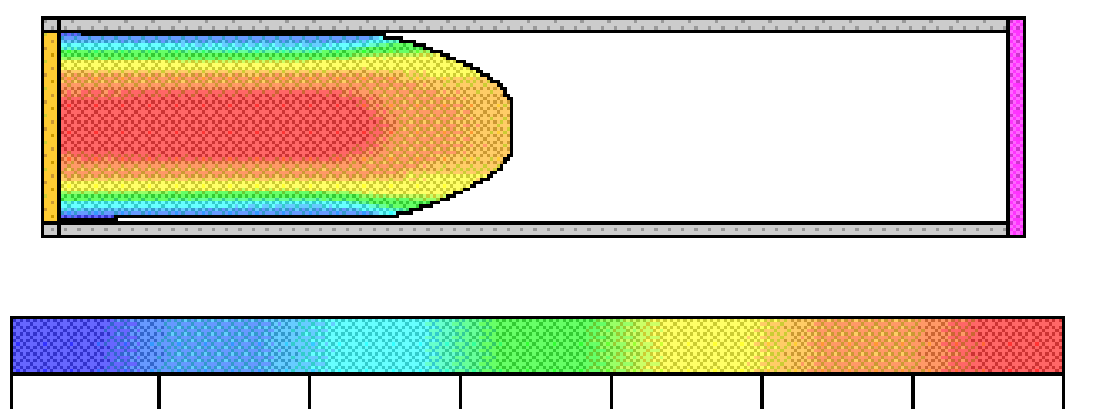

$\begin{array}{llllllll}-2.700 & -1.926 & -1.153 & -0.379 & 0.395 & 1.169 & 1.943 & 2.717\end{array}$

(b)

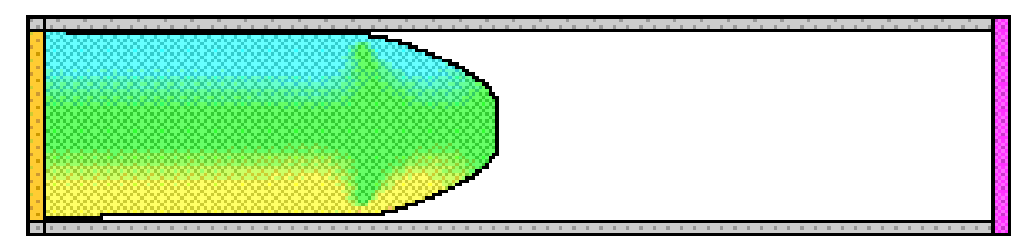

(c)
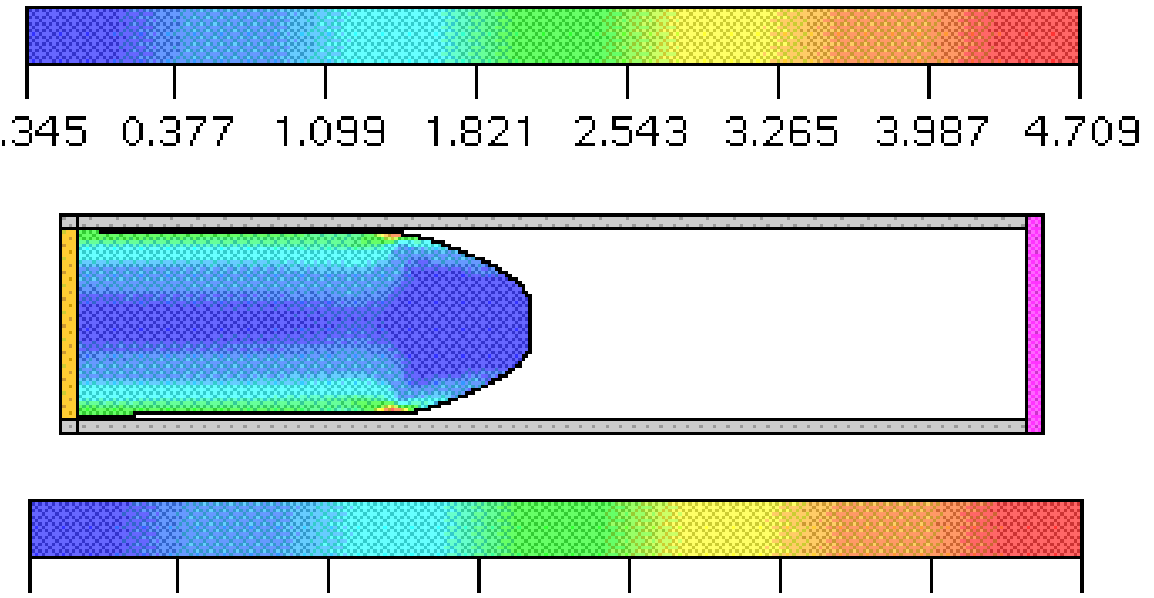

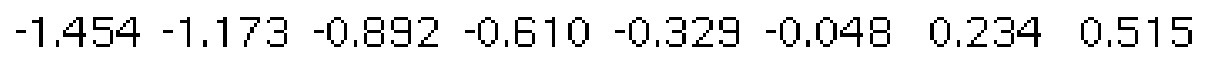

(d)

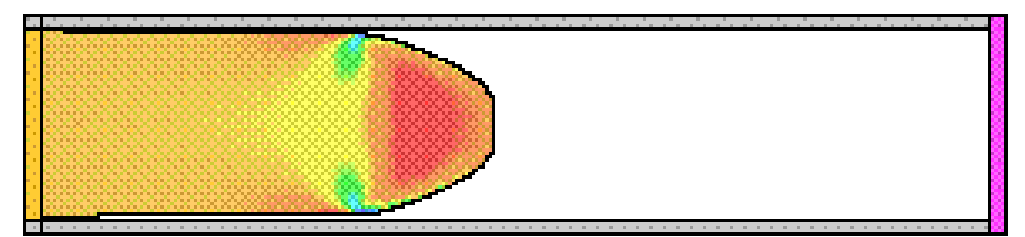

Figura 2.17: Simulação numérica do escoamento em um canal bidimensional em $t=7 \mathrm{~s}$. Isolinhas das variáveis: (a) $u$, (b) $\tau^{x y}$, (c) $\tau^{x x}$ e (d) $\tau^{y y}$. Dados de entrada: $R e=1, W e=1.25, \xi=$ $0.01, \varepsilon=0.5, \Delta \mathrm{p}_{\mathrm{x}}=-1.0$. 

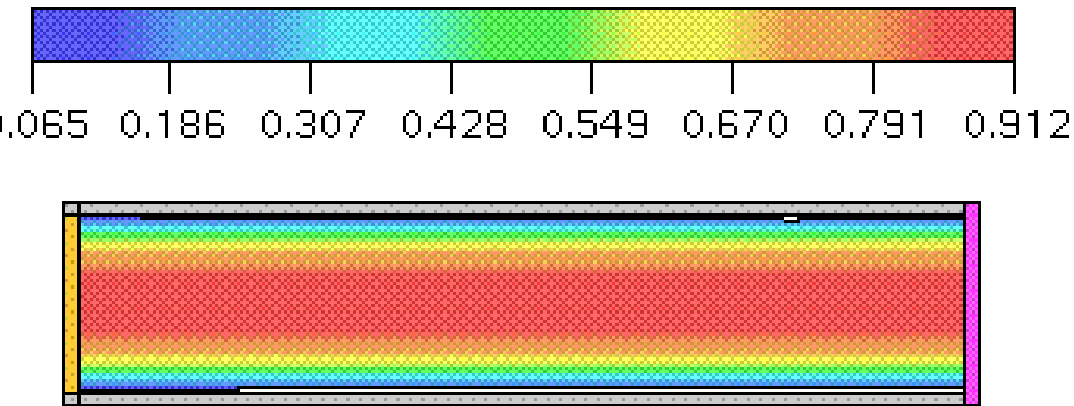

(a)
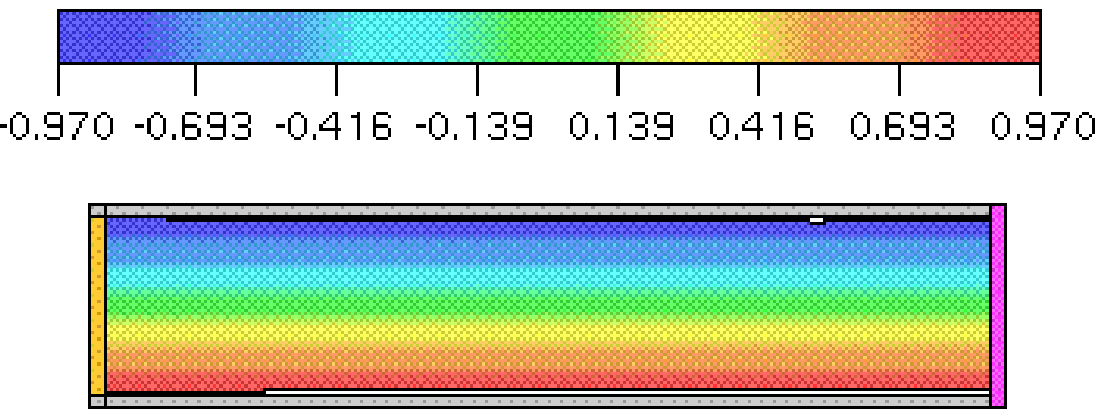

(b)
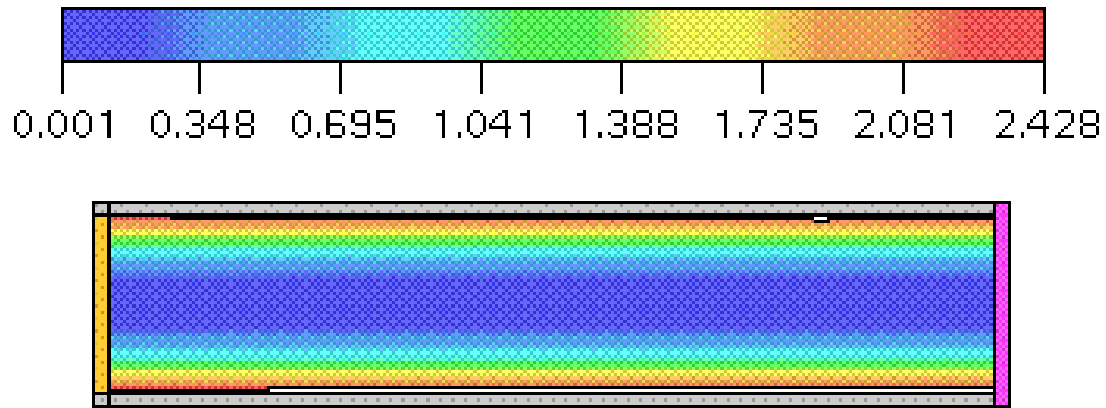

(c)
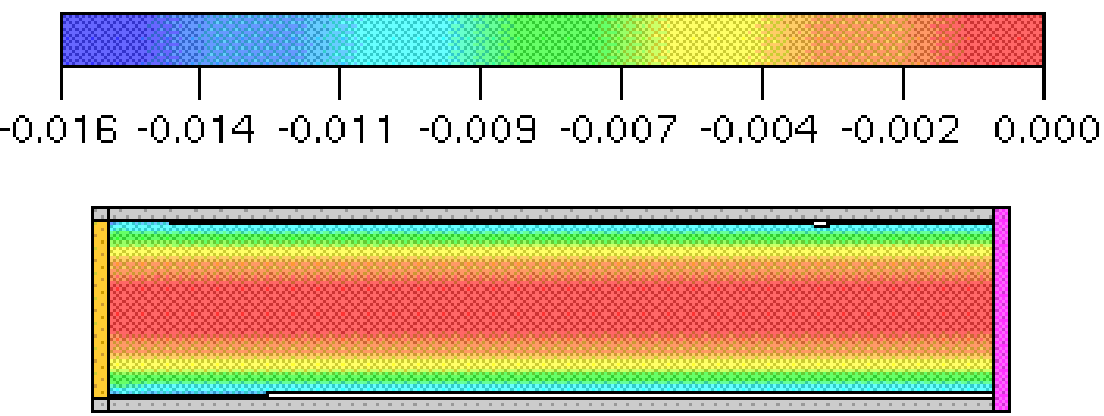

(d)

Figura 2.18: Simulação numérica do escoamento em um canal bidimensional em $t=100 \mathrm{~s}$. Isolinhas das variáveis: (a) $u$, (b) $\tau^{x y}$, (c) $\tau^{x x}$ e (d) $\tau^{y y}$. Dados de entrada: $R e=1$, We $=$ $1.25, \xi=0.01, \varepsilon=0.5, \Delta \mathrm{p}_{\mathrm{x}}=-1.0$. 

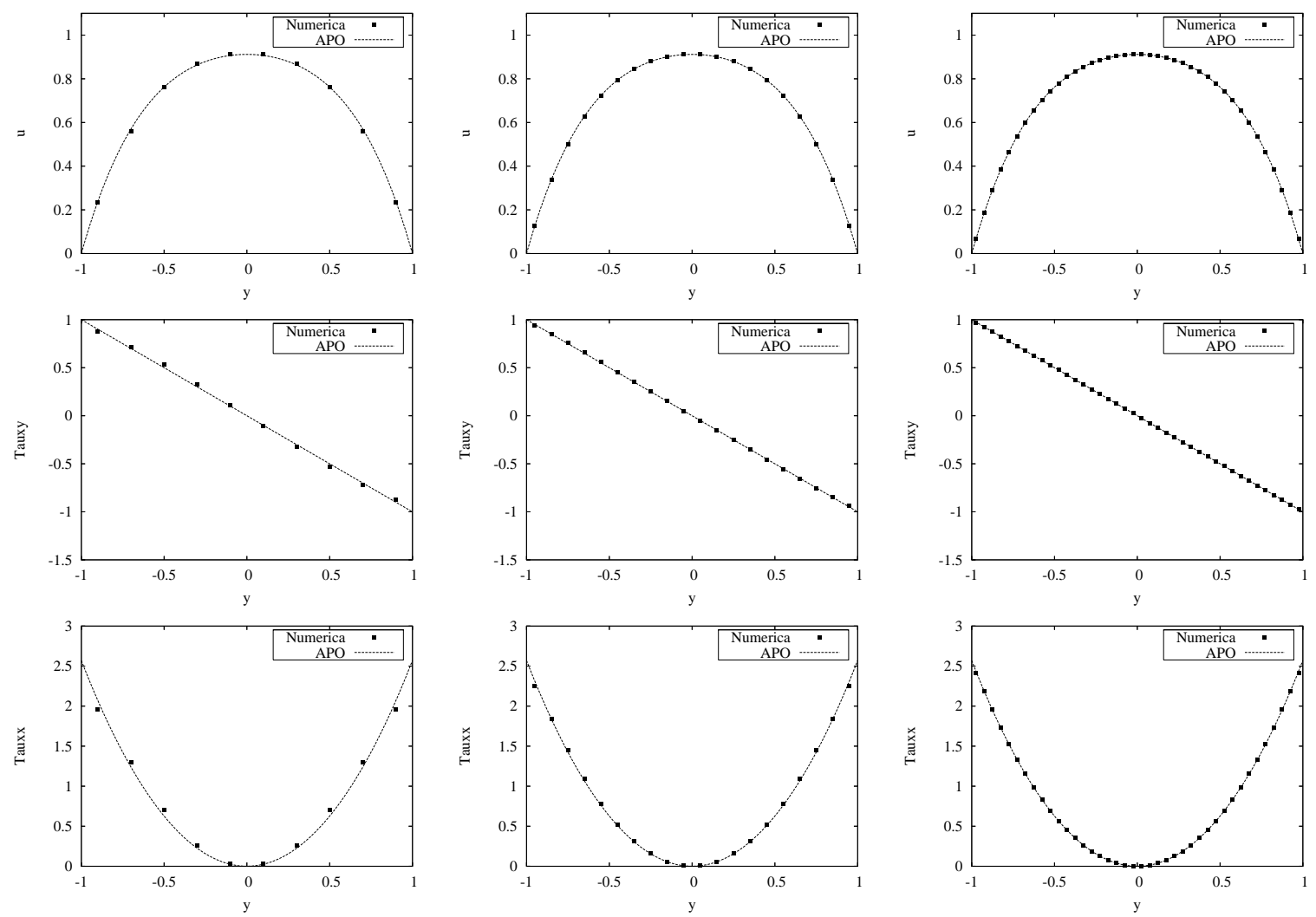

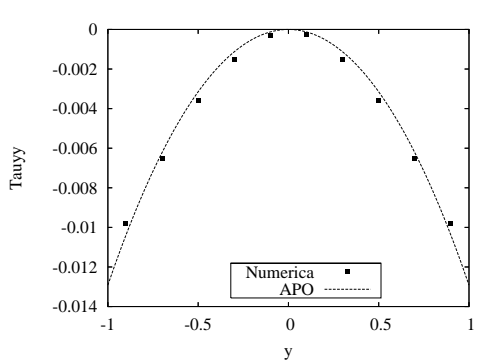

(a)

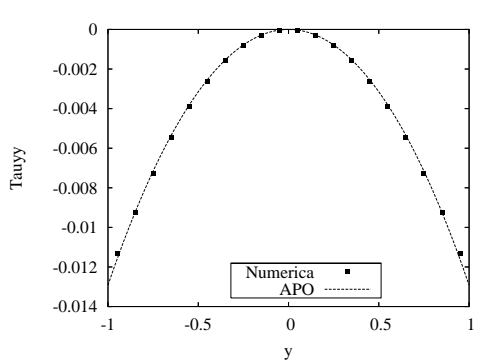

(b)

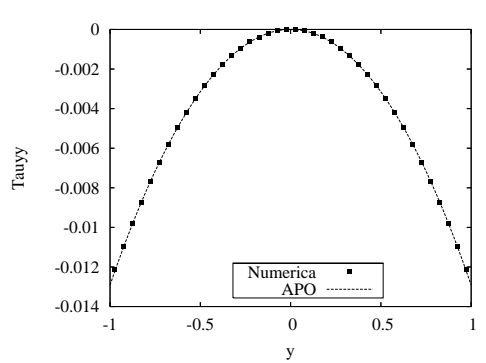

(c)

Figura 2.19: Solução numérica e solução analítica das componentes $u, \tau^{x y}, \tau^{x x}$ e $\tau^{y y}$ no meio do canal no tempo $t=100$ s. Coluna (a) M1, Coluna (b) M2, Coluna (c) M3 - Re $=1, W e=$ 1.25 .

Como a solução analítica da componente $\tau^{x x}$ do tensor extra-tensão não depende do parâmetro $\varepsilon$ presente no modelo PTT (ver equação (2.69)), uma segunda forma de verficação do método numérico desenvolvido nesta tese pode ser obtida fazendo a simulação do escoamento no canal utilizando dois valores diferentes da variável $\varepsilon$ e comparar os resultados numéricos obtidos para a variável $\tau^{x x}$ com a solução analítica. Com esse objetivo, apresentamos duas simulações com diferentes valores para $\varepsilon$ e comparamos estas soluções com a expressão analítica de $\tau^{x x}$ obtida com um terceiro valor para $\varepsilon$. Os resultados dessas simulações são mostrados na figura 2.20 . 


\begin{tabular}{|c|c|c|c|c|}
\hline Malha & $E(u)$ & $E\left(\tau^{x x}\right)$ & $E\left(\tau^{x y}\right)$ & $E\left(\tau^{y y}\right)$ \\
\hline M1 & $1.96410^{-2}$ & $2.24010^{-1}$ & $7.17410^{-2}$ & $1.24510^{-3}$ \\
\hline M2 & $1.38210^{-2}$ & $6.54710^{-2}$ & $1.62310^{-2}$ & $3.45810^{-4}$ \\
\hline M3 & $3.17510^{-3}$ & $1.07510^{-2}$ & $2.10610^{-3}$ & $5.23910^{-5}$ \\
\hline
\end{tabular}

Tabela 2.1: Erro relativo da solução numérica, dado pela norma $L_{2}$, em cada malha considerada.

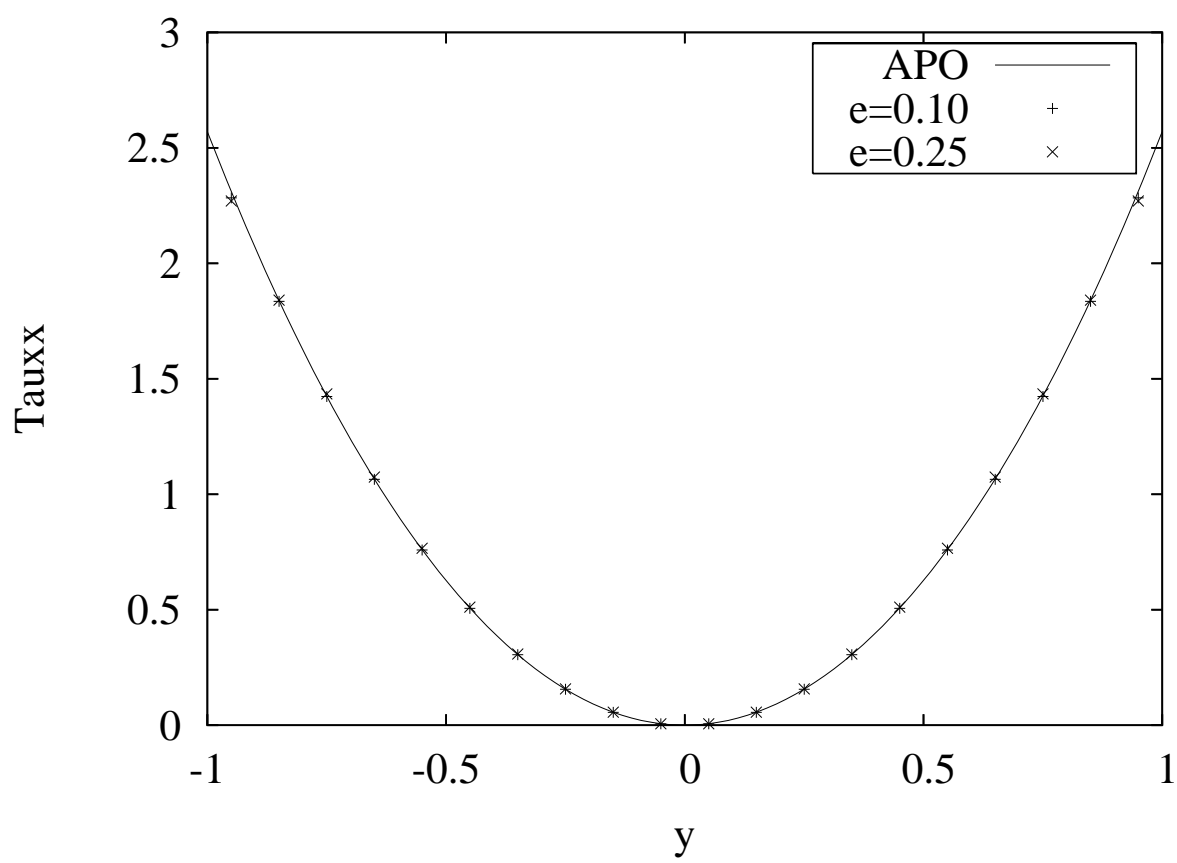

Figura 2.20: Componente $\tau^{x x}$. Solução analítica: APO. Solução numérica: $\varepsilon=0.1 ;$ e $\varepsilon=0.2$. $R e=1, W e=1.25, \xi=0.01, \Delta \mathrm{p}_{\mathrm{x}}=-1.0$.

A figura 2.20 apresenta o gráfico da solução analítica da componente $\tau^{x x}$ do tensor extra-tensão juntamente com os valores numéricos: um $\operatorname{com} \varepsilon=0.1$ e o outro $\operatorname{com} \varepsilon=0.2$. Podemos observar na figura 2.20 que a solução numérica independe de $\varepsilon$, e mais uma vez mostra que a solução numérica concorda com a solução analítica. 


\subsection{Simulação numérica de escoamentos viscoelásticos bidimensionais com superfícires livres}

Nesta Seção pretende-se demonstrar que o sistema Freeflow2D é capaz de simular escoamentos viscoelásticos com superfícies livres em movimento governados pela equação constitutiva não-linear PTT. A exemplo disso, consideramos o problema de um jato de fluido viscoelástico que ao incidir numa placa rígida pode apresentar o fênomeno conhecido como flambagem ou jato oscilante ('jet buckling') e o problema de um jato de fluido viscoelástico que ao ser expelido na atmosfera apresenta o fênomeno conhecido como inchamento do extrudado ('dieswell').

\subsubsection{Simulação numérica do jato oscilante}

No momento em que um jato incide sobre uma superfície rígida, se o número de Reynolds empregado for menor que um valor de referência então pode ocorrer o fênomeno conhecido como flambagem ou jato oscilante. Portanto, o número de Reynolds tem um caracter importante no comportamento do escoamento de um jato Newtoniano com relação ao fênomeno do jato oscilante. Entretanto, para um jato com fluido viscoelástico modelado pela equação constitutiva PTT, o número de Reynolds não é constante durante o escoamento pois o valor da viscosidade varia com o valor da taxa de deformação e com o tempo.

O fênomeno do jato oscilante tem atraído um grande número de pesquisadores e tem sido estudado tanto experimentalmente quanto numericamente. Cruickshank \& Munson [23] e Cruicksanck [22] apresentaram resultados teóricos e numéricos para jatos Newtonianos e obtiveram uma estimativa para quando o fenômeno do jato oscilante ocorre. A estimativa encontrada mostra uma relação entre o numéro de Reynolds e a razão $H / D$ onde $H$ é a altura do injetor até a superfície rigída e $D$ é o diâmetro do injetor. Esses autores demonstraram que um jato Newtoniano bidimensional sofrerá oscilações se as seguintes condições (2.73) forem satisfeitas:

$$
R e<0.56 \text { e } H / D>3 \pi
$$

Para jatos Newtonianos axissimétricos Cruickshank \& Munson mostraram que se as condições $(2.74)$

$$
R e<1.2 \text { e } H / D>2 \pi \text {. }
$$

forem satisfeitas então o jato oscilará. Tomé \& McKee [86] investigaram este problema numéricamente realizando simulações de jatos oscilantes bidimensionais. As simulações numéricas para o caso tridimensional foram feitas por Castelo et al. [15] (ver também Tomé et al. [79]). Neste caso, os autores simularam o escoamento de um jato tridimensional de um fluido Newtoniano utilizando um injetor com o formato de um paralelepípedo. Considerando fluidos viscoelásticos, a primeira investigação do grupo de pesquisa do LCAD (Laboratório Computacional de Alto Desempenho) do ICMC/USP com fluidos viscoelásticos foi feita por Tomé et al. [84] onde os autores estudaram o escoamento de um jato de fluido viscoelástico 
modelado pela equação constitutiva Oldroyd-B. Com o intuito de expandir a aplicabilidade do sistema Freeflow2D para escoamentos viscoelásticos vamos demonstrar nesta Seção que o método numérico GENSMAC2D-PTT é capaz de tratar o problema do jato oscilante para fluidos viscoelásticos governados pelo modelo PTT.

Para demonstrar esse fato vamos apresentar dois problemas onde ambos possuem a mesma geometria (ver figura 2.21) mas diferentes números de Reynolds. Os dados geométricos do problema do jato oscilante estão dispostos na Tabela 2.2 para melhor compreensão.

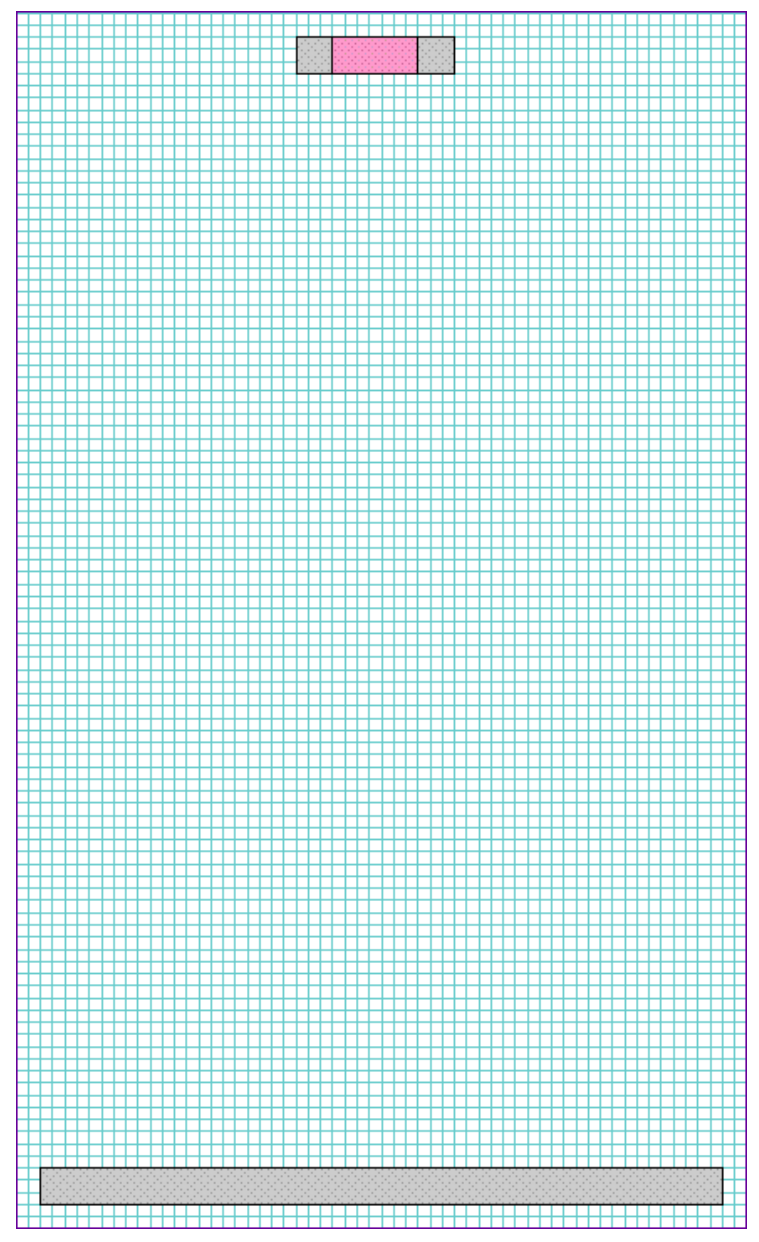

Figura 2.21: Domínio geométrico: $5.6 \times 9.6 \mathrm{~cm} ; 56 \times 96$ células. Placa rígida de $5.6 \mathrm{~cm}$. Injetor de $7 \mathrm{~mm}$

\section{Dados geométricos}

\begin{tabular}{|l|l|}
\hline domínio & $5.6 \mathrm{~cm} \times 9.6 \mathrm{~cm}$ \\
\hline malha & $56 \times 96$ células \\
\hline$H:$ altura do injetor até a placa rígida & $9 \mathrm{~cm}$ \\
\hline$D:$ diâmetro do injetor & $7 \mathrm{~mm}$ \\
\hline
\end{tabular}

Tabela 2.2: Modelagem do problema. Geometria utilizada para o problema I e problema II .

Pode-se observar na Tabela 2.2 que $H / D=12.85>3 \pi$. Dessa forma, escolhemos $R e=0.1$ para o problema I a ser simulado e $R e=0.7$ para o problema II, ou seja, escolhemos números 
de Reynolds de forma que para um problema a condição (2.73) está satisfeita (problema I) e para o outro (problema II) a condição (2.73) não é satisfeita. Cada problema consiste no estudo de duas simulações, uma caracterizada por um fluido Newtoniano e a outra por um fluido viscoelástico. Os dados que caracterizam os fluidos, tanto os Newtonianos quanto os viscoelásticos em cada problema, estão dispostos na Tabela 2.3.

Dados que caracterizam os escoamentos dos fluidos Newtoniano e PTT

\begin{tabular}{|l|l|l|}
\hline Newtoniano e PTT & Problema I $(R e=0.1)$ & Problema II $(R e=0.7)$ \\
\hline \hline$U:$ velocidade de injeção & $\mathbf{0 . 1 \mathrm { ms } ^ { - 1 }}$ & $\mathbf{0 . 7 \mathrm { ms } ^ { - 1 }}$ \\
\hline$g_{x}:$ força gravitacional & $-9.81 \mathrm{~ms}^{-2}$ & $-9.81 \mathrm{~ms}^{-2}$ \\
\hline$\nu=\eta / \rho$ & $0.007 \mathrm{~m}^{2} \mathrm{~s}^{-1}$ & $0.007 \mathrm{~m}^{2} \mathrm{~s}^{-1}$ \\
\hline$R e$ & $\mathbf{0 . 1}$ & $\mathbf{0 . 7}$ \\
\hline$F r$ & 0.381607 & 2.67125 \\
\hline modelo PTT & & \\
\hline$\lambda$ & $\mathbf{0 . 0 3 5 \mathrm { s }}$ & $\mathbf{0 . 0 0 5 \mathrm { s }}$ \\
\hline$\varepsilon$ & 0.01 & 0.01 \\
\hline$\xi$ & 0.01 & 0.01 \\
\hline$W e$ & 0.5 & 0.5 \\
\hline
\end{tabular}

Tabela 2.3: Simulação numérica do jato oscilante. Dados utilizados nas simulações com jatos Newtonianos e viscoelásticos.

Os resultados provenientes do problema I, ou seja, das simulações do jato Newtoniano e do jato viscoelástico onde $R e=0.1$, são apresentados na figura 2.22 em diferentes tempo. Podemos observar na figura 2.22 que ambos os jatos oscilam e o jato Newtoniano confirma a teoria de Cruickshank. Porém, o jato viscoelástico apresenta muito mais dobras que o jato Newtoniano.

Os resultados referentes ao problema II, ou seja, das simulações do jato Newtoniano e do jato viscoelástico com $R e=0.7$, são apresentados na figura $2.23 \mathrm{em}$ diferentes tempos. Como podemos observar na figura 2.23, o jato Newtoniano não sofre nenhuma oscilação ao atingir a placa rígida, confirmando mais uma vez a teoria de Cruickshank. Entretanto, o jato viscoelástico continua apresentando o fênomeno da oscilação e portanto podemos concluir que a teoria apresentada por Cruickshank não se aplica ao jato contendo fluido viscoelástico.

Acreditamos que o jato viscoelástico apresentou o efeito jato oscilante devido ao crescimento da viscosidade extensional a partir do momento em que o jato atinje a placa rígida. Com o objetivo de confirmar esse fato, na próxima seção realizamos várias simulações nas quais analizamos o efeito do número de Weissenberg no jato oscilante. 


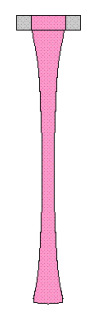

$t=0.30 \mathrm{~s}$
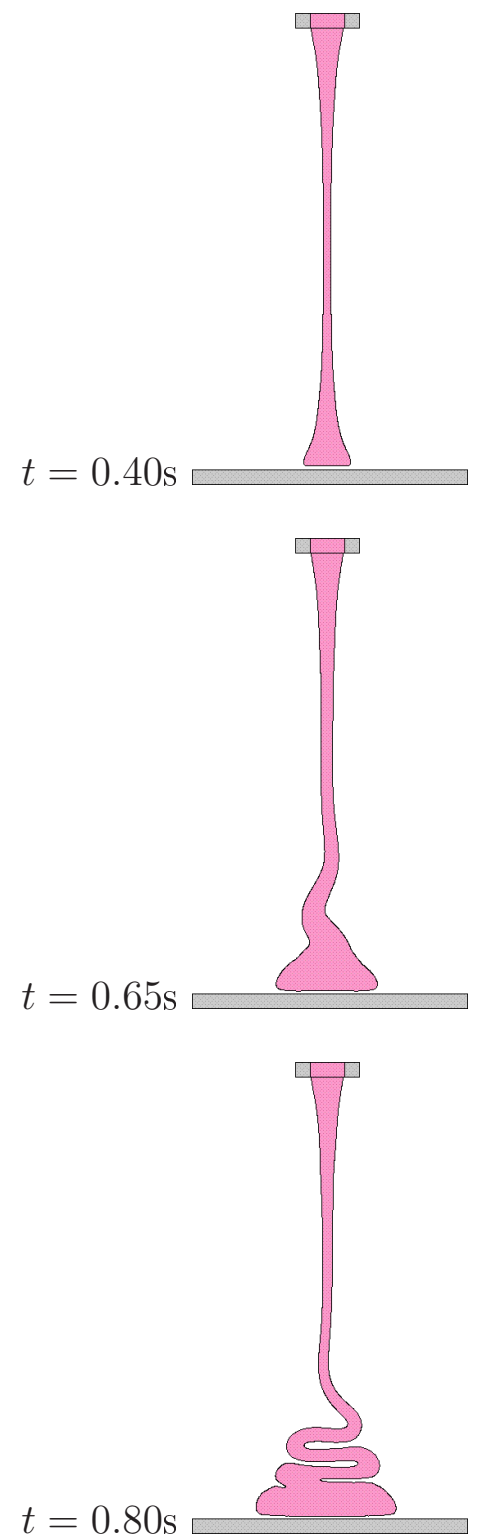
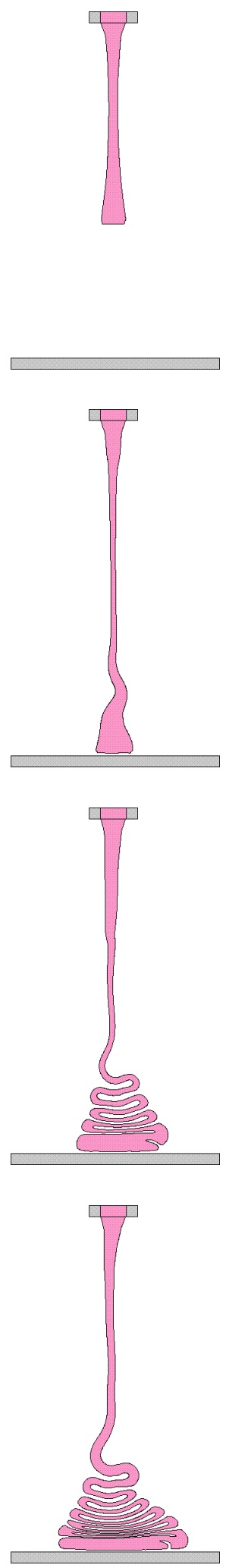

Figura 2.22: Simulação numérica do jato oscilante em diferentes tempos. Visualização do fluido. Coluna a esquerda - Jato Newtoniano, Coluna a direita - Jato PTT: $R e=0.1, W e=0.5$, $\xi=0.01$ e $\varepsilon=0.01$. 


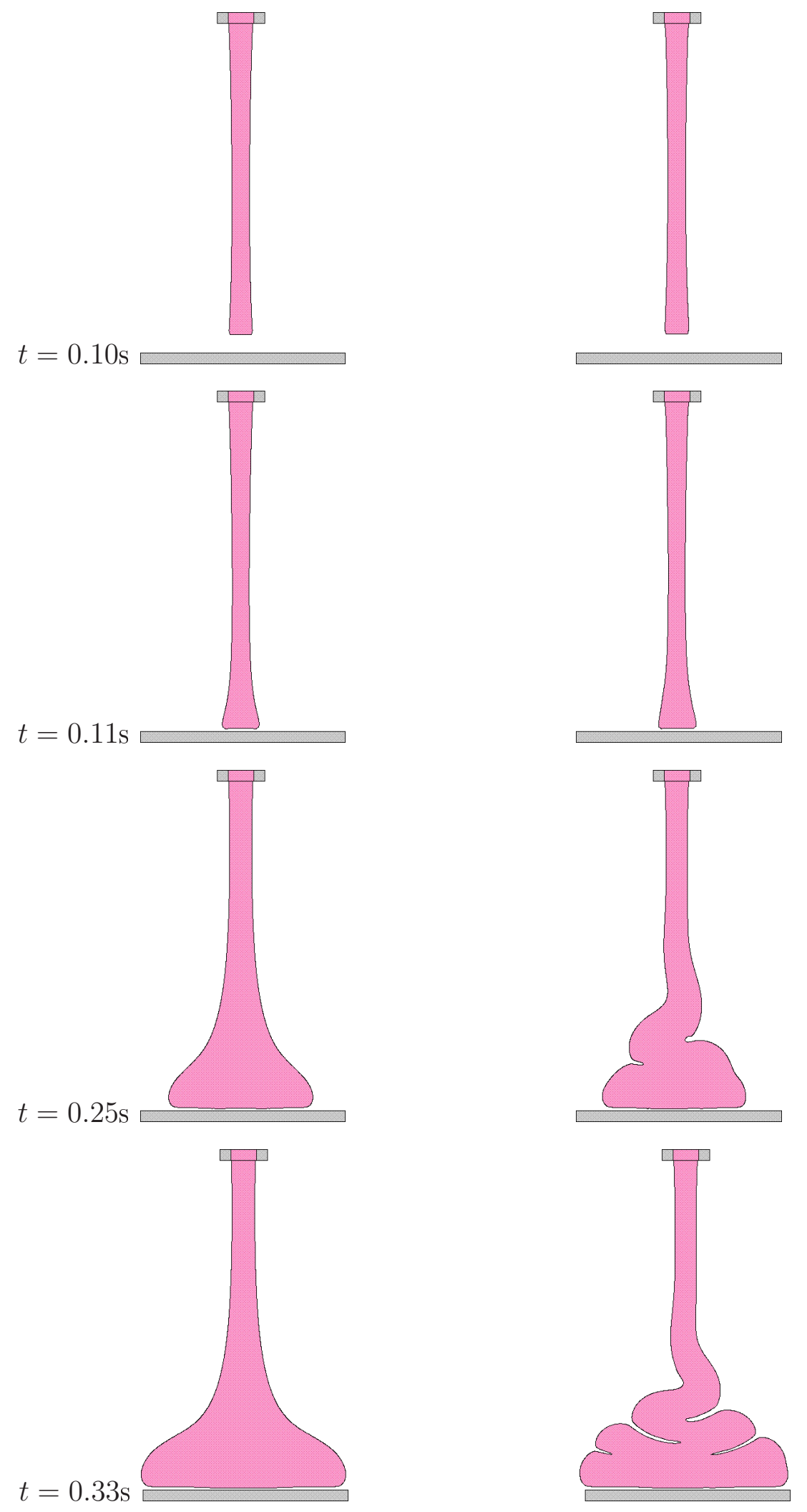

Figura 2.23: Simulação numérica do jato oscilante em diferentes tempos. Visualização do fluido. Coluna a esquerda - Jato Newtoniano, Coluna a direita - Jato PTT: $R e=0.7, W e=0.5$, $\xi=0.01$ e $\varepsilon=0.01$. 


\subsubsection{Efeito da viscoelásticidade no jato oscilante}

Para demonstrar que a viscoelásticidade, representada pelo número de Weissenberg, tem um efeito importante no problema do jato oscilante, realizamos várias simulações onde constatamos que para um dado número de Reynolds, se o número de Weissenberg for grande o suficiente então o jato ao incidir sobre uma placa rígida apresenta o fenômeno do jato oscilante. Além disso, vamos mostrar que para jatos viscoelásticos, a viscosidade extensional apresenta uma contribuição importante no efeito do jato oscilante.

Com relação aos problemas I e II, se considerarmos o jato escoando na direção da placa rígida, então, a viscosidade extensional pode ser calculada pela equação (conforme consta no livro de Makosko [46], ver também [12])

$$
\eta^{1}=\frac{\tau^{y y}-\tau^{x x}}{D^{y y}}
$$

a qual varia no decorrer do escoamento com o valor da taxa de deformação local e primeira diferença de tensões normais.

Observamos que entre o intervalo de tempo em que o jato é injetado até o tempo em que o mesmo atinge a placa rígida, temos um escoamento puramente extensional e a viscosidade do fluido pode ser calculada pela equação (2.75).

Para constatarmos que a viscosidade extensional assume valores elevados após o jato atingir a placa rígida, consideramos os problemas I e II novamente e calculamos a viscosidade extensional em um pouco específico no jato. Consideramos o eixo de simetrica do jato e fixamos uma altura como mostra a figura 2.24 e calculamos os valores da viscosidade extensional dada pela equação (2.75) em relação ao tempo t. O intervalo de tempo escolhido é uma vizinhança do tempo em que o jato atinge a placa rígida. Após o jato atingir a placa rígida temos então um escoamento complexo, envolvendo também cizalhamento. No problema I o jato atinge a placa rígida em $t=38$ s aproximadamente e no problema II em aproximadamente $t=0.11$ s. Os valores obtidos para a viscosidade extensional são mostrados nas figuras 2.25(b) e 2.26(b) durante os intervalos de tempos considerados para cada problema.

As figuras 2.25(a) e 2.26(a) também mostram a viscosidade dos fluidos Newtonianos nos intervalos de tempo considerados. A viscosidade $\eta^{1}$ dos fluidos Newtonianos pode ser calculada utilizando a equação constitutiva $\tau=\frac{2}{R e} \mathbf{D}$ e a equação de conservação de massa, as quais fornecem:

$$
\eta^{1}=\frac{\tau^{y y}-\tau^{x x}}{D^{y y}}=\frac{\frac{2}{R e} \frac{\partial v}{\partial y}-\frac{2}{R e} \frac{\partial u}{\partial x}}{\frac{\partial v}{\partial y}}=\frac{4}{R e}=\left\{\begin{array}{cll}
40 & \text { se } & R e=0.1 \\
5.71428 & \text { se } & R e=0.7
\end{array}\right.
$$

Os valores numéricos obtidos para as viscosidades Newtonianas são mostradas nas figuras 2.25(a) e 2.26(a) e concordam com valores exatos fornecidos pela equação (2.76). 
Em resumo, podemos observar nas figuras 2.25(b) e 2.26(b), que a viscosidade dos fluidos viscoelásticos está variando durante o intervalo de tempo considerado e portanto, a teoria de Cruickshank não pode ser aplicada aos jatos viscoelásticos. Podemos observar também que a viscosidade extensional aumenta após o jato atingir a placa rígida o que faz com que o número de Reynolds local diminua dificultando a mobilidade do fluido e consequentemente o jato oscila.

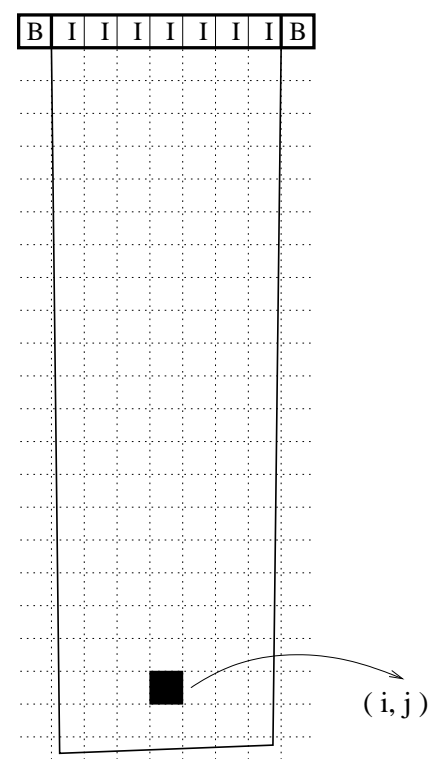

Figura 2.24: Cálculo da viscosidade extensional: o ponto em destaque representa a célula $(i, j)=(31,80)$ do domínio computacional. Esta célula está localizada no eixo de simetria do jato.

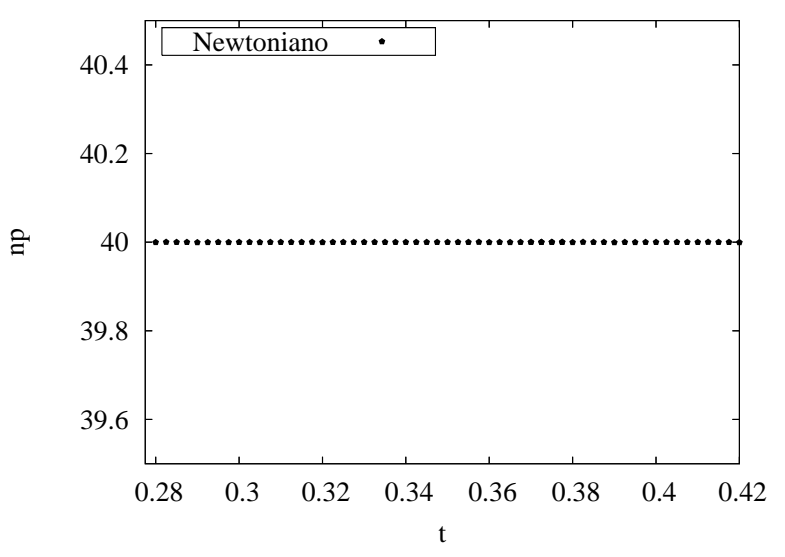

(a)

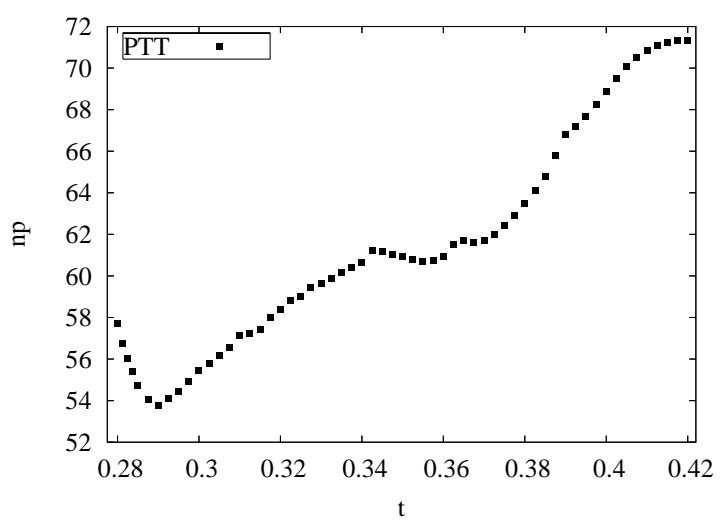

(b)

Figura 2.25: Gráfico da viscosidade $\eta^{1}$ no ponto $(i, j)=(31,80)$ em função do tempo $t$. Re $=$ $0.1, W e=0.5, \xi=0.01$ e $\varepsilon=0.01$. (a) Fluido Newtoniano. (b) Fluido viscoelástico (modelo PTT). 


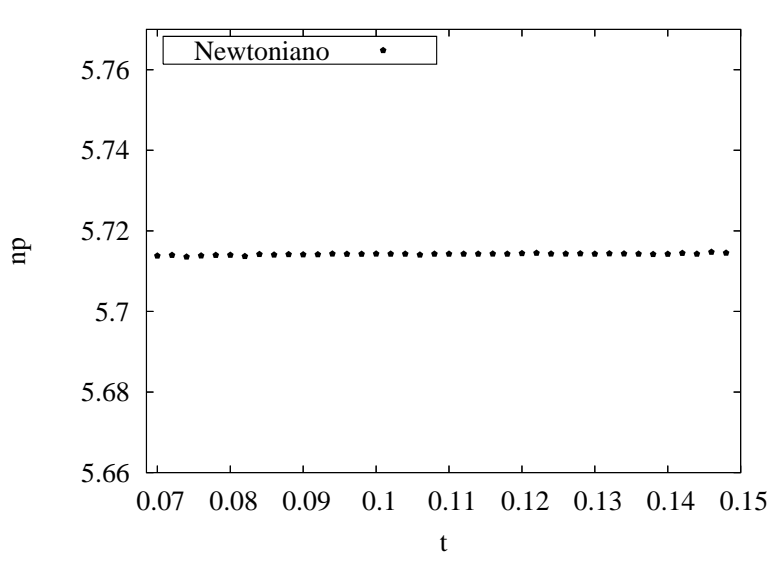

(a)

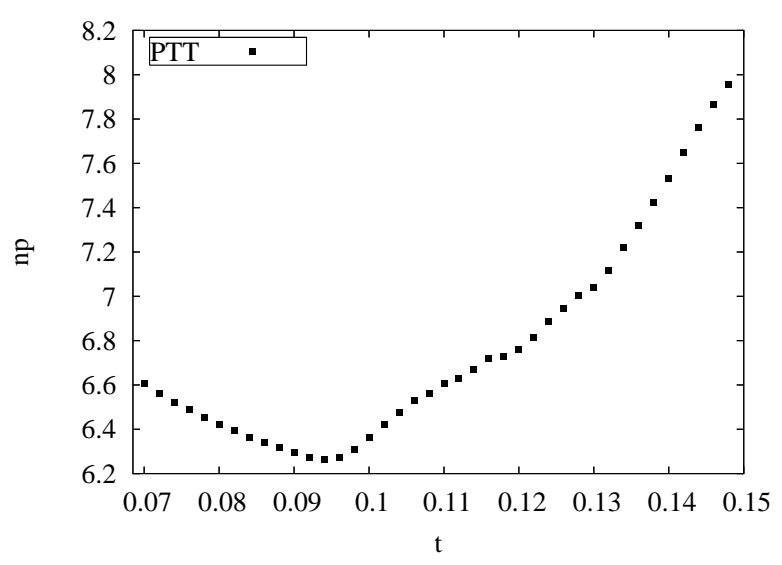

(b)

Figura 2.26: Gráfico da viscosidade $\eta^{1}$ no ponto $(i, j)=(31,80)$ em função do tempo $t$. $R e=$ $0.7, W e=0.5, \xi=0.01$ e $\varepsilon=0.01$. (a) Fluido Newtoniano. (b) Fluido viscoelástico (modelo PTT).

Dando continuidade aos estudos do fênomeno do jato oscilante, foram realizadas várias simulações com o objetivo de encontrar um valor de referência do número de Reynolds para o qual o jato com fluido viscoelástico não apresenta o efeito oscilatório. Nessas simulações foram utilizadas a mesma geometria empregada nos problemas I e II e foram mantidos fixos os parâmetros $\varepsilon, \xi$ e $W e=0.5$. Três simulações foram realizadas para os seguintes valores do número de Reynolds: $R e=0.8,0.9,1.0$. Os dados utilizados nessas simulações são os mesmos da Tabela 2.3, exceto a velocidade de escala $(U)$ que foi ajustada para termos os números de Reynolds $0.8,0.9,1.0$ e a constante temporal $\lambda$ foi calculada para que o número de Weissenberg permanecesse fixo em $W e=0.5$. O resultados dessas simulações para $t=0.25$ s são mostrados na figura 2.27 .

(a)

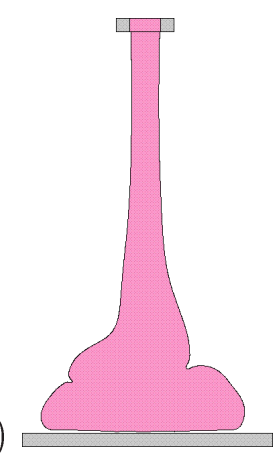

(b)

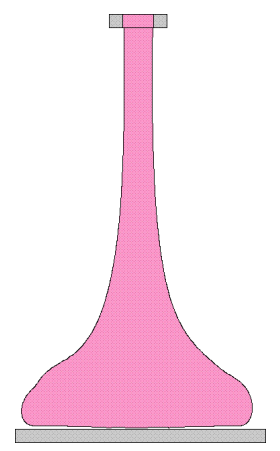

(c)

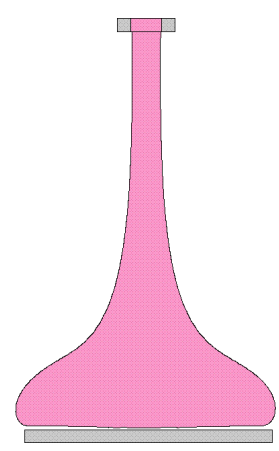

Figura 2.27: Simulação numérica do jato oscilante no tempo $t=0.25 \mathrm{~s}: ~ W e=0.5, \xi=0.01 \mathrm{e}$ $\varepsilon=0.01$. (a) $R e=0.8$ (b) $R e=0.9$ (c) $R e=1$. 


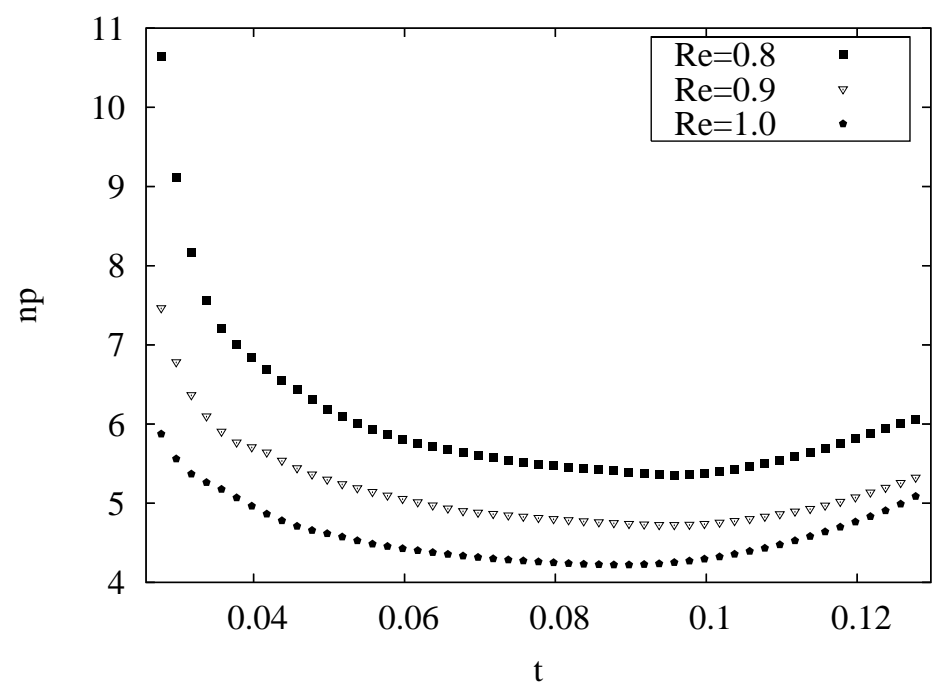

Figura 2.28: Graficos da viscosidade $\eta^{1}$ no ponto $(i, j)=(31,80)$ em relação ao tempo $t$ para os jatos viscoelásticos com $R e=0.8, R e=0.9$ e $R e=1$ : $W e=0.5, \xi=0.01$ e $\varepsilon=0.01$ fixos.

Podemos observar na figura 2.27(a) que o jato com $R e=0.8$ apresentou o efeito do jato oscilante enquanto que o jato com $R e=0.9$ (ver figura 2.27(b)) apresentou uma leve assimetria e o jato com $R e=1$ (ver figura $2.27(\mathrm{c})$ ) não apresentou nenhum sinal de assimetria. Estes resultados mostram que a inclusão de inércia no escoamento permite que o fluido escoe com maior mobilidade e pode suprimir o efeito do jato oscilante em fluidos viscoelásticos. Para constatar essa afirmação calculamos os valores da viscosidade extensional, dada pela equação (2.75) em função do tempo $t$ na célula mostrada na figura 2.24 para as três simulações. Nestas simulações os jatos atingem a placa rígida nos tempos $t=0.75 \mathrm{~s}$ e $t=0.95$ s aproximadamente e os valores de $\eta^{1}$ são mostrados na figura 2.28 no intervalo de tempo $t \in[0.02,0.13]$. Da figura 2.28, podemos observar que quanto maior é o número de Reynolds nas simulações, menor é o valor da viscosidade. Esse resultado concorda com os mostrados na figura 2.27.

Vimos anteriormente (ver figura 2.27) que para a geometria adotada nos problemas I e II e para $W e=0.5$, o jato oscila sempre que $R e<1$. Com o objetivo de investigar o efeito da viscoelasticidade no problema do jato oscilante, realizamos várias simulações onde mantivemos o número de Reynolds constante e variamos o número de Weissenberg. Empregamos a mesma geometria utilizada nos problemas I e II, fixamos o número de Reynolds em $R e=1$ e fizemos várias simulações aumentando continuamente o número de Weissenberg até os resultados mostrarem o efeito do jato oscilante. Os dados utilizados no modelo PTT foram os mesmos utilizados nos resultados mostrados na figura 2.27, ou seja, $\varepsilon=0.01$ e $\xi=0.01$. A constante temporal $\lambda$ foi ajustada de modo a obtermos os números de Weissenberg $W e=0.6,0.7,0.8,0.9,1.0,1.2,1.3$. No total foram realizadas sete simulações e os resultados correspondentes a $W e=1.3$ mostraram que o jato após incidir sobre a placa rígida apresenta o efeito do jato oscilante. De fato, a figura 2.29 mostra a configuração do fluido em $t=0.24 \mathrm{~s}$ para $W e=0.8 .1 .2,1.3$ e podemos observar na figura $2.29 \mathrm{a}$, para $W e=0.8$ o jato escoa lateralmente e não apresenta o efeito jato oscilante e na figura $2.29 \mathrm{~b}$, correspondente a $W e=1.2$, o jato sofre uma pequena assimetria e na figura 
$2.29 \mathrm{c}$, correspondente a $W e=1.3$, o jato apresentou o efeito do jato oscilante. Esses resultados demonstram que o aumento da viscoelasticidade tem um efeito importante no problema do jato oscilante.

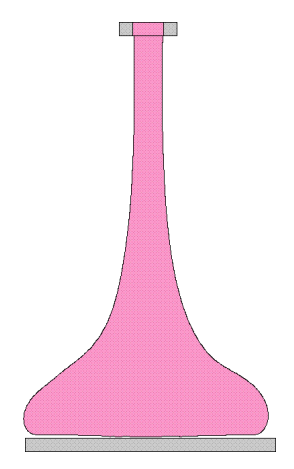

(a)

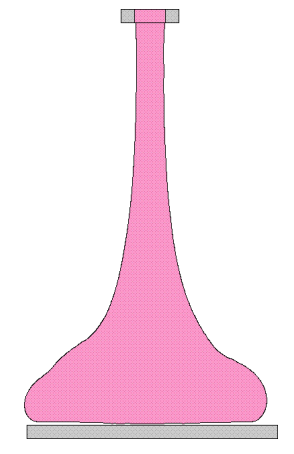

(b)

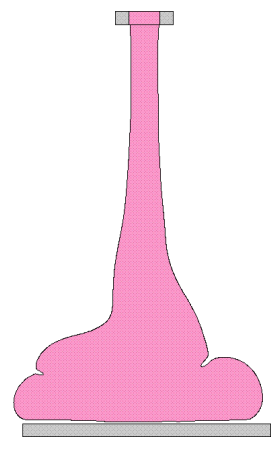

(c)

Figura 2.29: Jatos de fluidos viscoelásticos no tempo $t=0.24 \mathrm{~s}: R e=1.0, \xi=0.01$ e $\varepsilon=0.01$ nos três casos. (a) $W e=0.9$ (b) $W e=1.2 \quad$ (c) $W e=1.3$. 


\subsubsection{Teste de convergência: refinamento da malha}

Para analisar a convergência de GENSMAC2D-PTT no problema do jato oscilante, simulamos esse problema em três diferentes malhas. Os dados utilizados nessas três simulações estão dispostos na Tabela 2.4 .

\section{Dados geométricos}

\begin{tabular}{|l|l|}
\hline domínio & $5.6 \mathrm{~cm} \times 10.6 \mathrm{~cm}$ \\
\hline$H$ & $10 \mathrm{~cm}$ \\
\hline$D$ & $5 \mathrm{~mm}$ \\
\hline
\end{tabular}

\section{Dados que caracterizam o fluido}

\begin{tabular}{|l|l|}
\hline modelo PTT & $R e=0.25$ e $W e=1$ \\
\hline \hline$U:$ velocidade de injeção & $0.5 \mathrm{~ms}^{-1}$ \\
\hline$g_{x}:$ força & $-9.81 \mathrm{~ms}^{-2}$ \\
\hline$\nu=\eta / \rho$ & $0.01 \mathrm{~m}^{2} \mathrm{~s}^{-1}$ \\
\hline$R e$ & 0.25 \\
\hline$F r$ & 2.257618 \\
\hline$\lambda$ & $0.01 \mathrm{~s}$ \\
\hline$\varepsilon$ & 0.1 \\
\hline$\xi$ & 0.1 \\
\hline$W e$ & 1 \\
\hline
\end{tabular}

\section{Malhas utilizadas}

\begin{tabular}{|l|l|l|r|}
\hline Malha 0 (M0) & $56 \times 106$ & $\delta x=\delta y=0.00100$ & 5 células no injetor \\
\hline Malha 1 (M1) & $112 \times 212$ & $\delta x=\delta y=0.00050$ & 10 células no injetor \\
\hline Malha 2 (M2) & $224 \times 424$ & $\delta x=\delta y=0.00025$ & 20 células no injetor \\
\hline
\end{tabular}

Tabela 2.4: Dados utilizados na simulação do jato oscilante em diferentes malhas.

Utilizando os dados da Tabela 2.4, o problema do jato oscilante foi simulado nas malhas M0, M1 e M2 e a figura 2.30 mostra a configuração da superfície livre do jato em diferentes tempos. Podemos observar nas figuras 2.30(a), 2.30(b) e 2.30(c) uma boa concordância entre os resultados numéricos obtidos nas três malhas. Em particular, a figura 2.30(d) mostra que a solução obtida na malha mais grossa M0 apresenta uma oscilação um pouco mais acentuada que acreditamos seja devido aos erros de arredondamento. Em geral, podemos afirmar que esses resultados demonstram que o método GENSMAC2D-PTT converge quando a malha é refinada. 

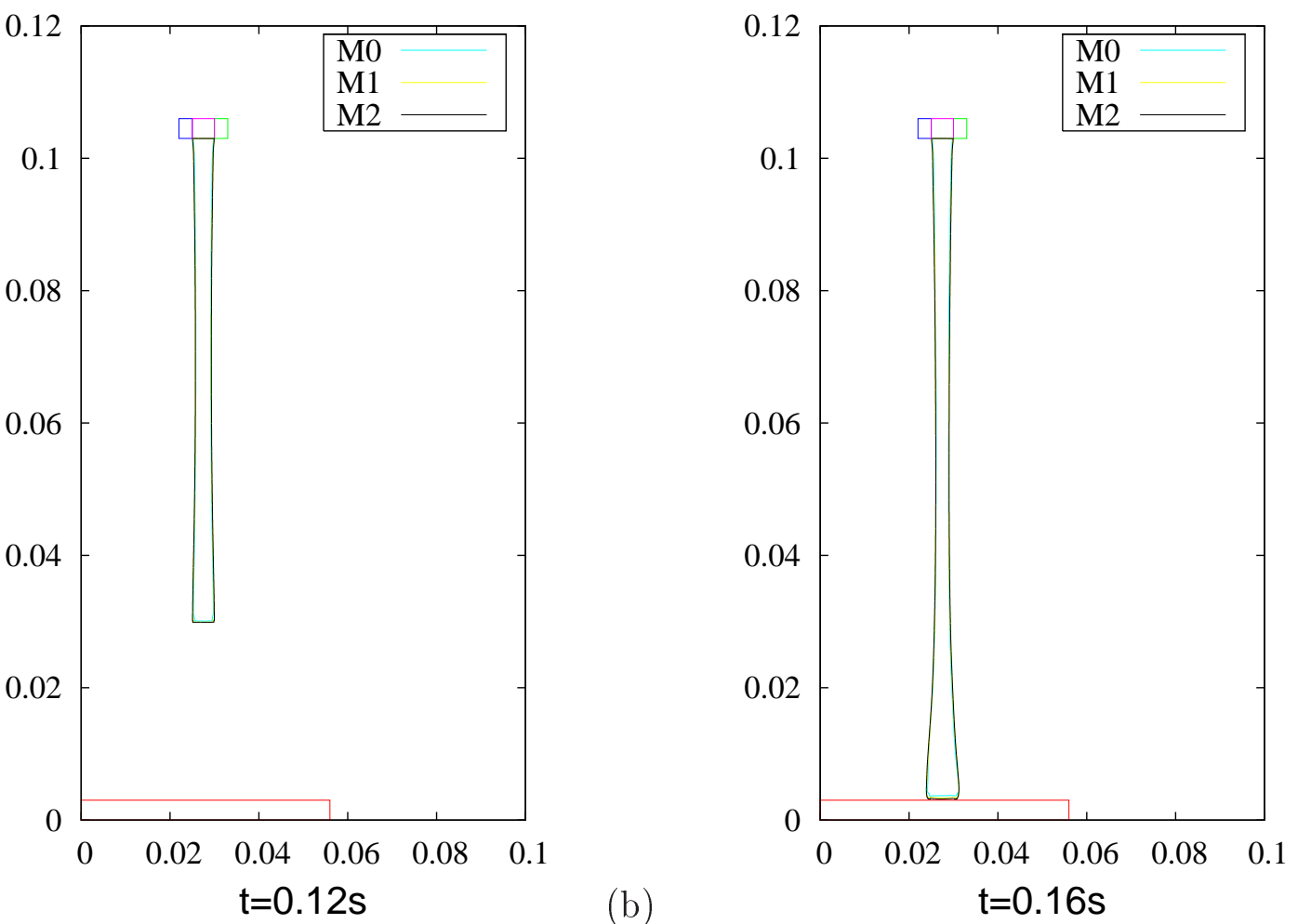

(a)
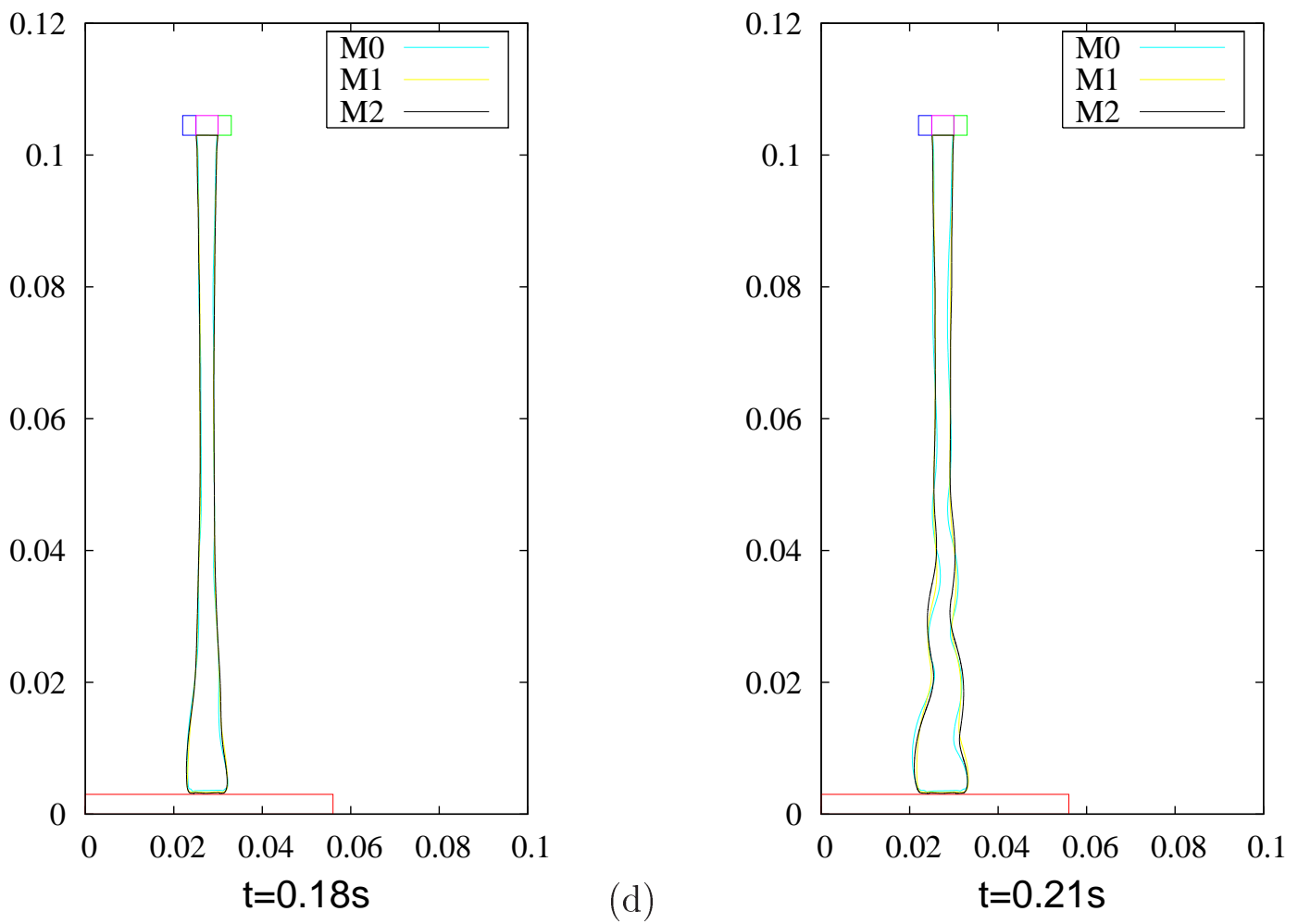

Figura 2.30: Simulação numérica do jato oscilante nas malhas M0, M1 e M2. Dados utilizados: $R e=0.25, W e=1, \xi=0.1$ e $\varepsilon=0.1$. Configuração da superfície livre do jato em diferentes tempos: (a) $t=0.12 \mathrm{~s}$, (b) $t=0.16 \mathrm{~s}$, (c) $t=0.18 \mathrm{~s}, \quad$ (d) $t=0.21 \mathrm{~s}$. 


\subsubsection{Simulação numérica do inchamento do extrudado}

No momento em que um jato é expelido na atmosfera pode ocorrer um fênomeno conhecido como inchamento do extrudado, que é caracterizado pelo aumento do diâmetro do extrudado em relação ao diâmetro interno do tubo do qual o jato é expelido (ver figura 2.31). Esse fenômeno aparece em muitas atividades diárias, por exemplo, quando pressiona-se um frasco de shampoo, um tubo de creme dental, um frasco de detergente, etc. Nesses casos, pode ocorrer que o diâmetro do jato expelido é maior do que o oríficio do frasco por onde o fluido foi expelido. Usando água ao invés dos componentes citados acima, o inchamento é tão pequeno que não é perceptivel ao olho humano. Embora este fenômeno seja comum no dia-a-dia, o inchamento do extrudado é um fenômeno complexo e dificil de se descrever teoricamente e numericamente. Em geral, esse fênomeno ocorre devido a uma associação de efeitos que incluem relaxação da tensão normal na saída de fluido e gradientes de temperatura ao longo do tubo. Devido a aplicação em problemas industriais, o problema do inchamento do extrudado tem atraído a atenção de vários pesquisadores e várias técnicas numéricas para simular este problema tem sido propostas (ver por exemplo, [77, 68, 21, 38, 39, 84]).

O inchamento do extrudado é calculado pela relação entre a dilatação sofrida pelo jato após emergir na atmosfera e a largura do canal que o fluido ocupava (ver figura 2.31). Assim, define-se a taxa de inchamento $S_{r}$ como sendo $S_{r}=\frac{D_{\max }}{D}$. Esse problema tem sido investigado experimentalmente e sabe-se que para jatos de fluidos viscoelásticos, o inchamento do extrudado pode atingir altas taxas de inchamento, por exemplo, $S_{r}>100 \%$.

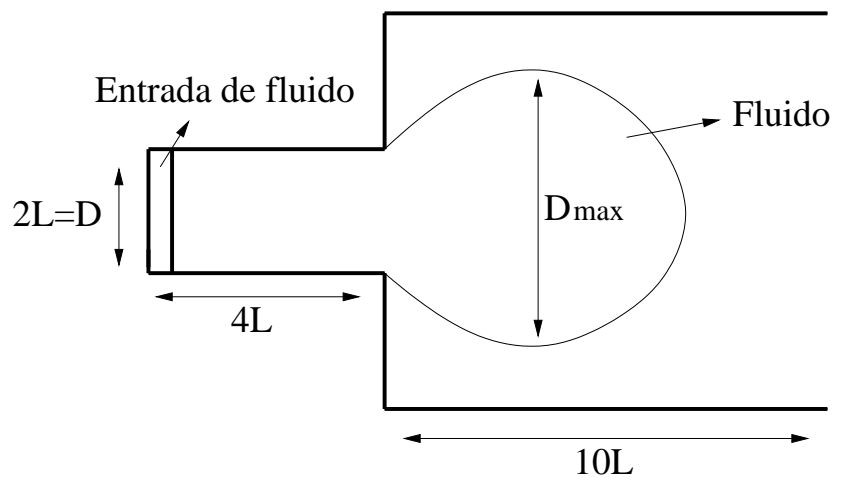

Figura 2.31: Definição do domínio computacional para a simulação numérica do inchamento do extrudado

Com o objetivo de demonstrar que GENSMAC2D-PTT pode simular escoamentos de fluidos altamente viscoelásticos, o problema do inchamento do extrudado foi simulado utilizando vários números de Weissenberg. Para simular esse problema, considere um jato escoando dentro de um canal bidimensional sendo expelido para a atmosfera conforme mostrado na figura 2.31. Na entrada do canal impomos as soluções analíticas do modelo PTT para escoamentos totalmente desenvolvidos em um canal bidimensional. Essas soluções analíticas são fornecidas na Seção anterior pelas equações (2.67)-(2.70). Nas paredes do canal a condição não-escorregamento é 
imposta e na superfície livre do fluido impomos as condições de contorno conforme as equações (2.14) e (2.15). Os dados geométricos do problema estão dispostos na Tabela 2.5 e o domínio computacional é ilustrado na figura 2.32 .

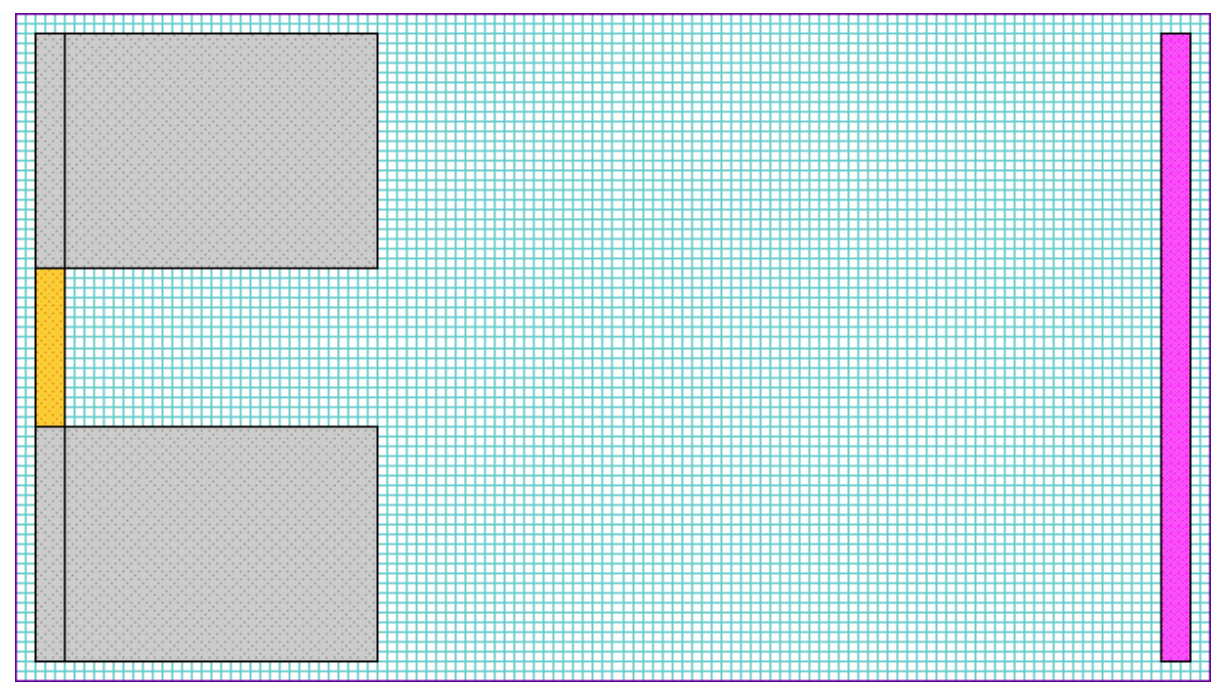

Figura 2.32: Simulação numérica do inchamento do extrudado. Domínio geométrico: $14.75 \mathrm{~m} \times$ $8.0 \mathrm{~m} ; 118 \times 64$ células.

Os parâmetros de escala nessas simulações foram $L, U, \nu$ e $\lambda$, e os valores utilizados são mostrados nas Tabelas 2.6 e 2.7. Foram realizadas simulações para os números de Reynolds $R e=0.1$ e $R e=1$, e em cada caso foram adotados diferentes números de Weissenberg. Utilizando $R e=1$ foram obtidas simulações para os seguintes números de Weissenberg, $W e=$ $0.1,0.5,1.0,1.5,2.0,2.5,3.0,3.5$ e para $R e=0.1$ foram obtidas simulações para os seguintes números de Weissenberg, $W e=0.1,0.5,1.0,1.5,2.0,2.5$. Os números de Weissenberg máximos obtidos para esse problema foram $W e=3.5$ para $R e=1$ e $W e=2.5$ para $R e=0.1$. Os dados que caracterizam os escoamentos dos fluidos viscoelásticos para cada uma das simulações estão dispostos nsa Tabelas 2.6 e 2.7. Os seguintes parâmetros foram mantidos constante em todas as simulações, $\varepsilon=\xi=0.01$ e $\Delta \mathrm{p}_{\mathrm{x}}=\partial p / \partial x=-0.65$.

\section{Dados geométricos}

\begin{tabular}{|l|l|}
\hline domínio & $10.75 \mathrm{~cm} \times 8.0 \mathrm{~m}$ \\
\hline malha & $86 \times 64$ células \\
\hline injetor $D=2 \mathrm{~L}$ & $2 \mathrm{~m}$ \\
\hline ejetor & $8 \mathrm{~m}$ \\
\hline distância do injetor ao ejetor $5 D=10 \mathrm{~L}$ & $10 \mathrm{~m}$ \\
\hline
\end{tabular}

Tabela 2.5: Simulação numérica do inchamento do extrudado. Modelagem do problema.

A figura 2.33 mostra os resultados das simulações do inchamento do extrudado com $R e=1$ e $W e=0.1,2.0,3.0$ nos tempos $t=40 \mathrm{~s}, 60 \mathrm{~s}, 75 \mathrm{~s}, 125 \mathrm{~s}, 250 \mathrm{~s}$ e $t=800 \mathrm{~s}$. No tempo $t=40 \mathrm{~s}$ a maior parte dos jatos mantêm-se dentro do canal, não sendo possível observar as diferenças oriundas da variação do número de Weissenberg, apenas o fato de $U_{\text {max }}$ variar um pouco em 


\begin{tabular}{|c|c|c|c|c|c|c|c|c|}
\hline$W e$ & 0.1 & 0.5 & 1 & 1.5 & 2 & 2.5 & 3 & 3.5 \\
\hline \hline$U=U_{\max }\left[\mathrm{ms}^{-1}\right]$ & 0.325 & 0.325 & 0.327 & 0.331 & 0.336 & 0.343 & 0.352 & 0.364 \\
\hline$\nu=\eta \rho\left[\mathrm{m}^{2} \mathrm{~s}^{-1}\right]$ & 0.325 & 0.325 & 0.327 & 0.331 & 0.336 & 0.343 & 0.352 & 0.364 \\
\hline$\lambda[\mathrm{s}]$ & 0.307 & 1.535 & 3.051 & 4.527 & 5.944 & 7.280 & 8.512 & 9.611 \\
\hline
\end{tabular}

Tabela 2.6: Simulação numérica do inchamento do extrudado. Dados utilizados nas simulações com $R e=1.0$.

\begin{tabular}{|c|c|c|c|c|c|c|}
\hline$W e$ & 0.1 & 0.5 & 1 & 1.5 & 2 & 2.5 \\
\hline \hline$U=U_{\max }\left[\mathrm{ms}^{-1}\right]$ & 0.500 & 0.502 & 0.510 & 0.523 & 0.544 & 0.575 \\
\hline$\nu=\eta \rho\left[\mathrm{m}^{2} \mathrm{~s}^{-1}\right]$ & 5.000 & 5.024 & 5.101 & 5.237 & 5.446 & 5.757 \\
\hline$\lambda[\mathrm{s}]$ & 0.199 & 0.995 & 1.960 & 2.863 & 3.671 & 4.342 \\
\hline
\end{tabular}

Tabela 2.7: Simulação numérica do inchamento do extrudado. Dados utilizados nas simulações com $R e=0.1$.

cada caso. Porém, a partir do tempo $t=60$ s podemos observar uma variação no inchamento do jato em relação ao número de Weissenberg. 

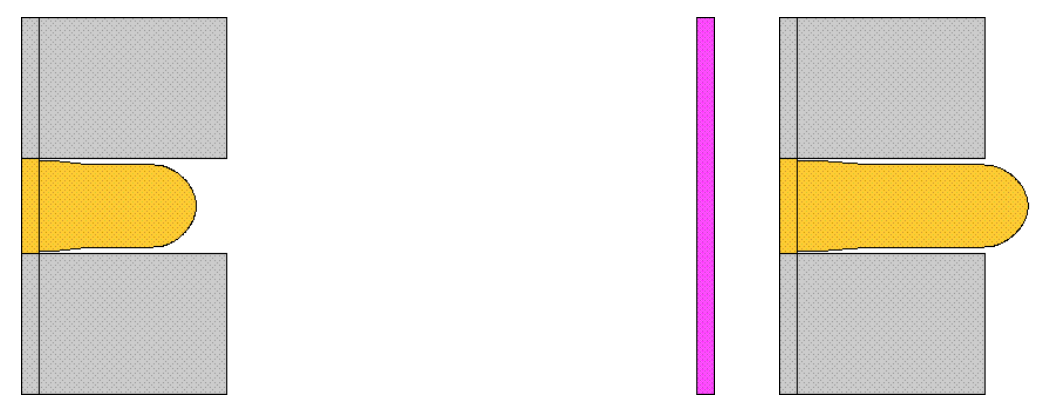

$W e=0.1$
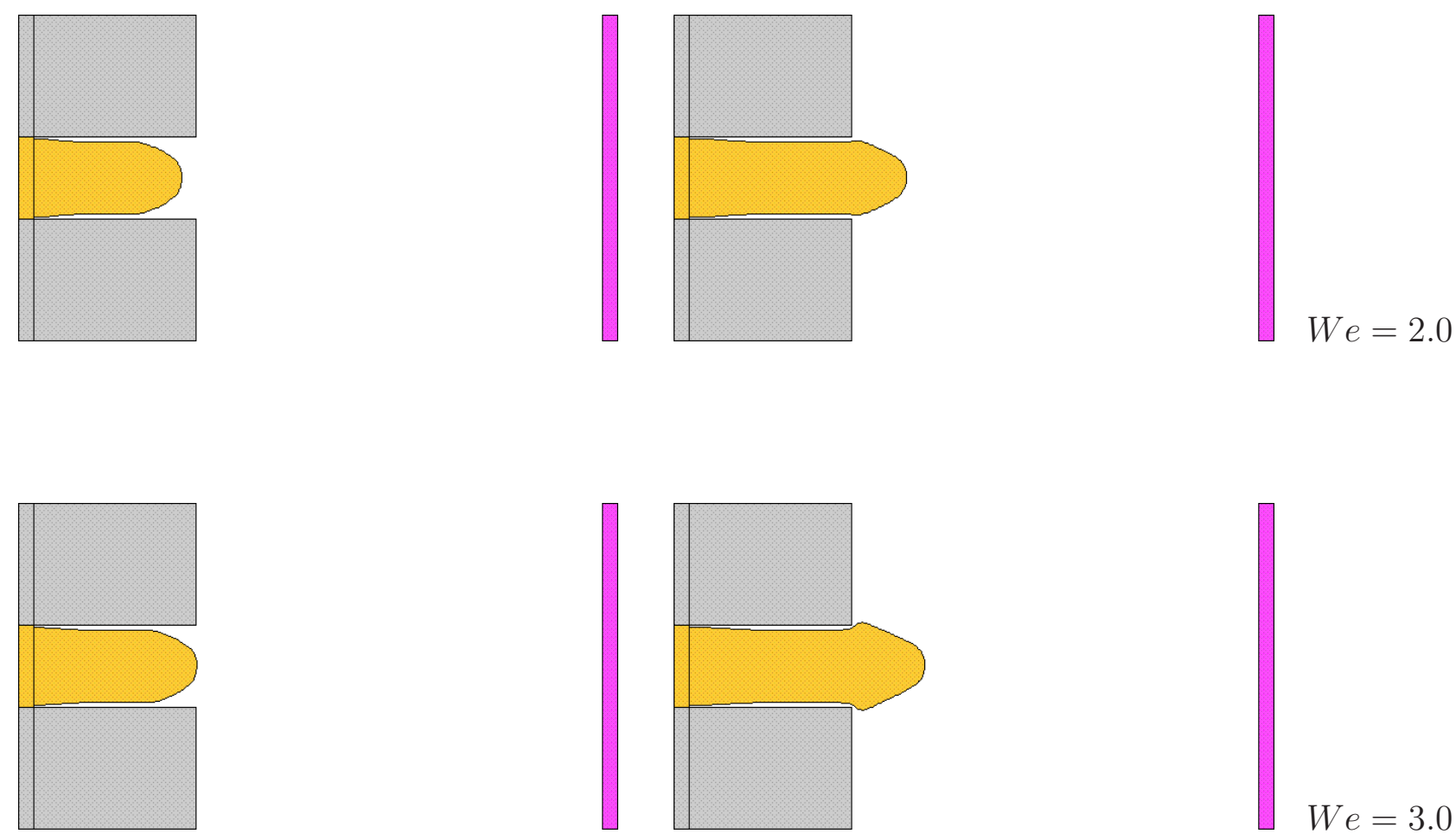

$$
t=40 \mathrm{~s}
$$$$
t=60 \mathrm{~s}
$$

Figura 2.33: Simulação do inchamento do extrudado: $R e=1$ e $W e=0.1,2.0,3.0$. Visualização do escoamento em diferentes tempos da simulação. 

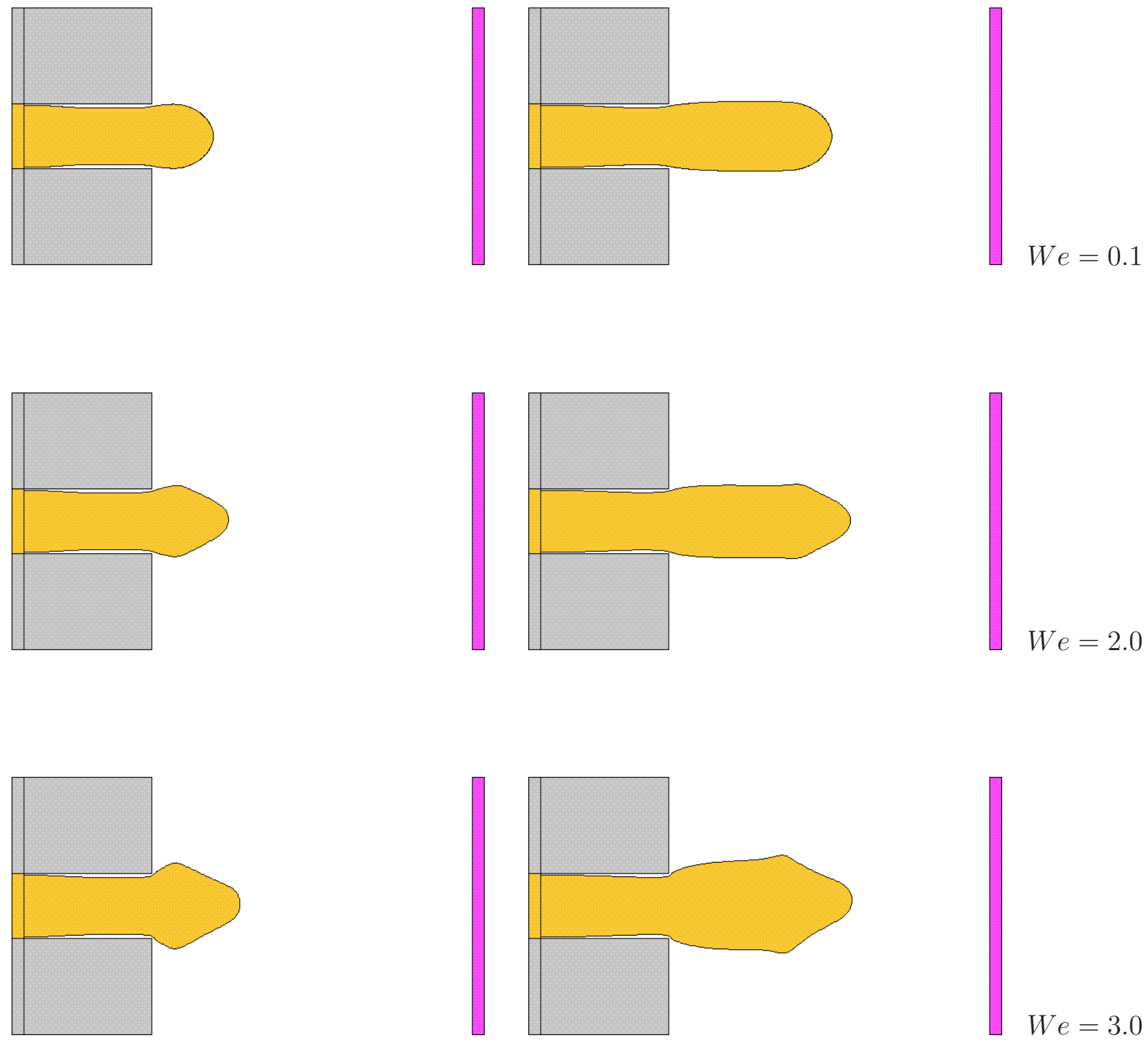

$$
t=75 \mathrm{~s}
$$$$
t=125 \mathrm{~s}
$$

Figura 2.33.Continuação. 

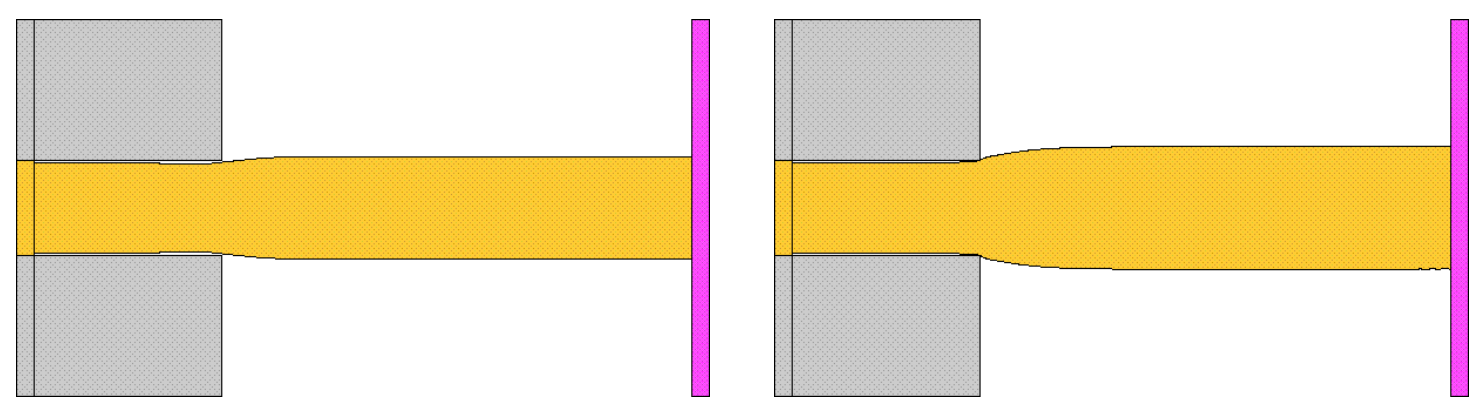

$W e=0.1$
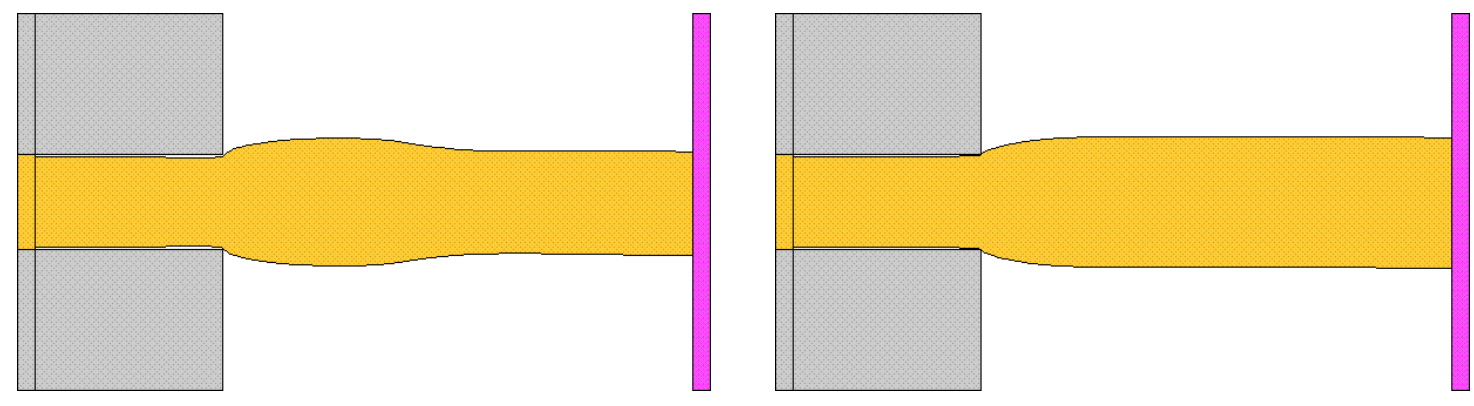

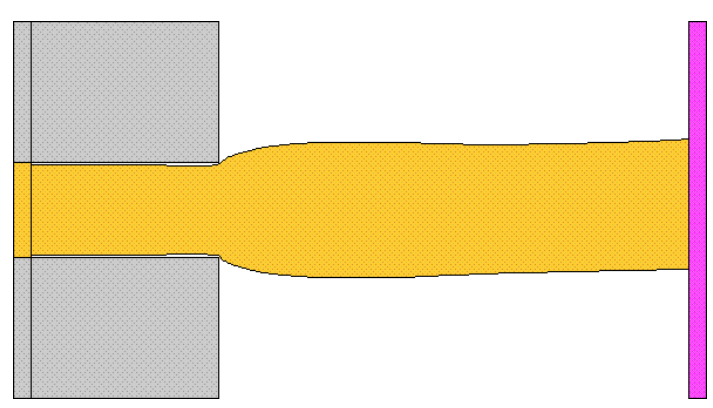

$t=250 \mathrm{~s}$
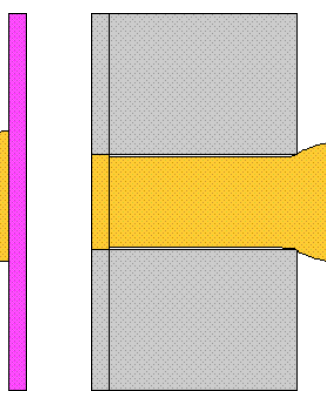

Figura 2.33. Continuação.
$W e=2.0$
$W e=3.0$

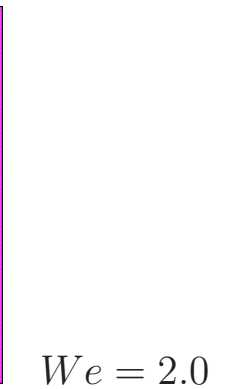

$t=800 \mathrm{~s}$ 
O gráfico mostrado na figura 2.34 mostra a variação da taxa de inchamento do extrudado obtida na simulações com $R e=1$ para cada valor de $W e$. O gráfico mostra que a razão de inchamento $S_{r}$ varia de aproximandamente $30 \%$, no caso em que $W e=0.1$ até $52 \%$, no caso em que $W e=3.5$.

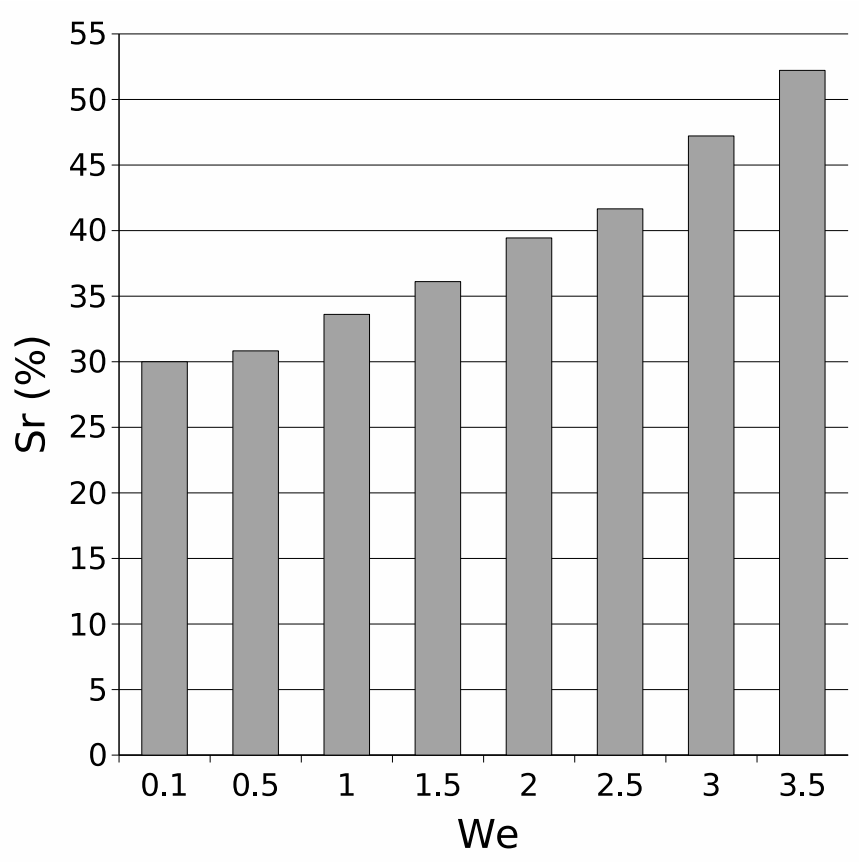

Figura 2.34: Razão de inchamento. $R e=1$.

A figura 2.35 mostra os resultados obtidos nas simulações do inchamento do extrudado com $R e=0.1$ e $W e=0.1,1.0,2.0$ nos tempos $t=25 \mathrm{~s}, 45 \mathrm{~s}, 75 \mathrm{~s}$ e $t=500 \mathrm{~s}$. No tempo $t=25 \mathrm{~s}$ os jatos já foram expelidos para a atmosfera, mas ainda não é possível observar as diferenças oriundas da variação do número de Weissenberg, apenas o fato do $U_{\max }$ variar um pouco em cada caso. A partir do tempo $t=45$ s podemos notar uma variação no inchamento do jato em relação ao número de Weissenberg. Os valores da taxa de inchamento obtidos para cada $W e$ são mostrados na figura 2.36 . 

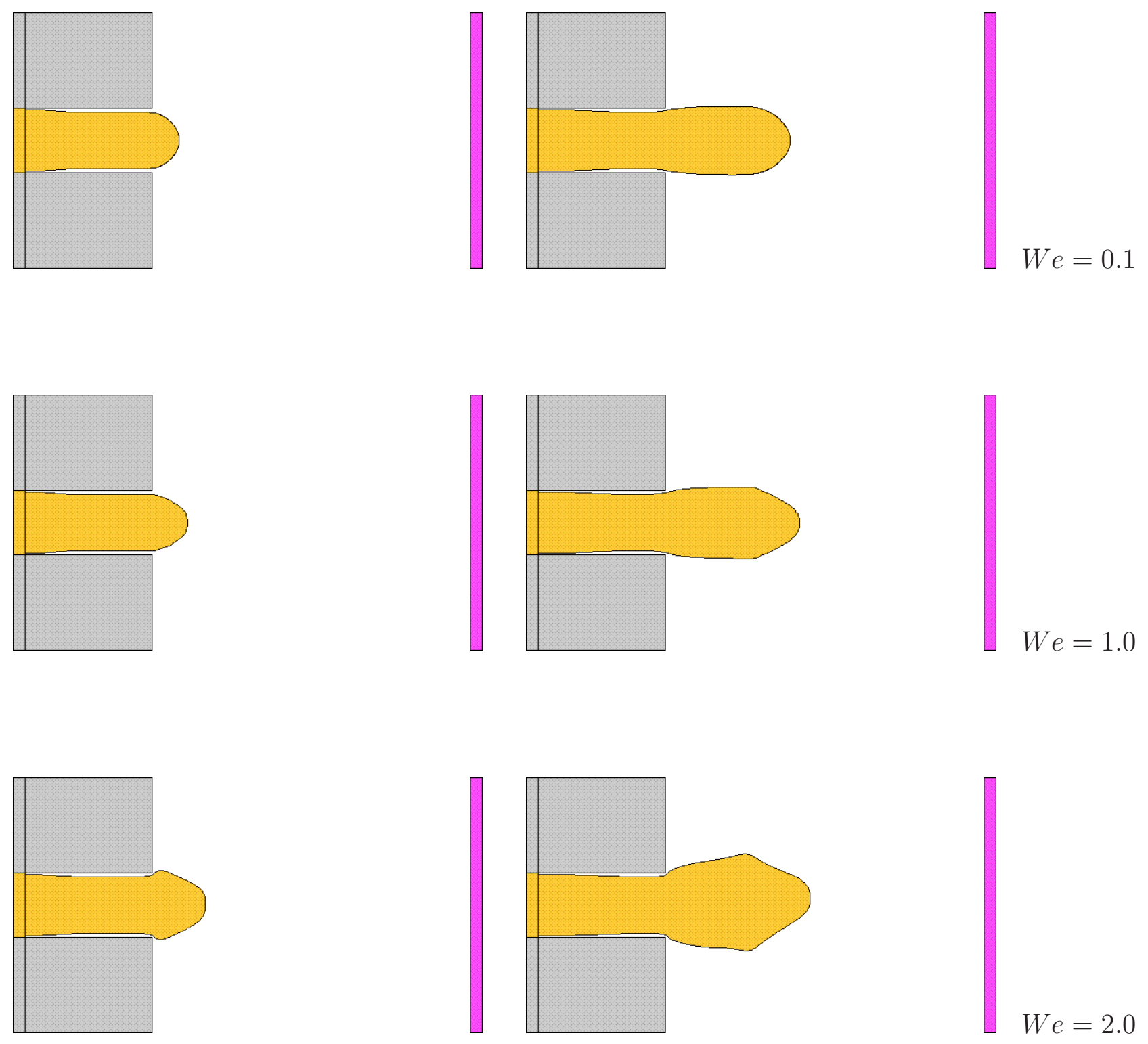

$$
t=25 \mathrm{~s}
$$

$$
t=45 \mathrm{~s}
$$

Figura 2.35: Simulação do inchamento do extrudado: $R e=0.1$ e $W e=0.1,1.0,2.0$. Visualização do escoamento em diferentes tempos da simulação. 

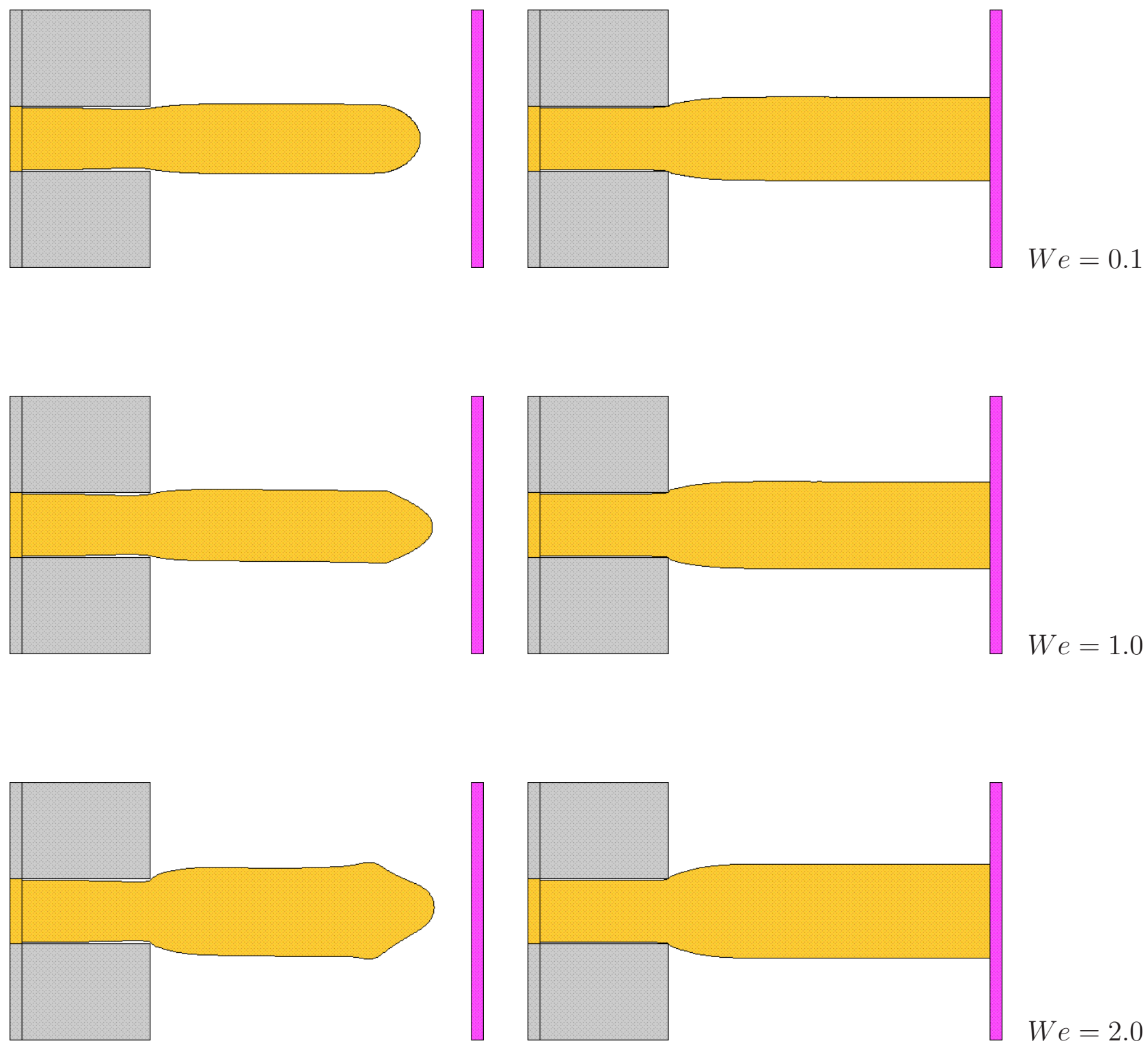

$t=75 \mathrm{~s}$

$t=500 \mathrm{~s}$

Figura 2.35. Continuação. 
A figura 2.36 mostra que a taxa de inchamento $S_{r}$ varia de aproximandamente $30.55 \%$, no caso em que $W e=0.1$ até $52.77 \%$, no caso em que $W e=2.5$ no caso em que $R e=0.1$.

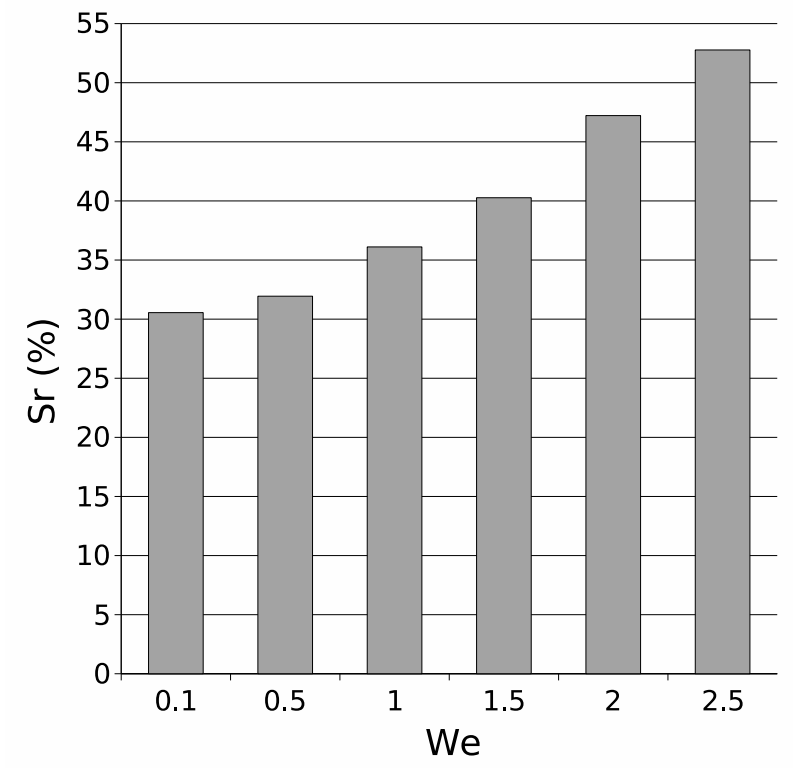

Figura 2.36: Razão de inchamento. $R e=0.1$.

Os resultados obtidos para a taxa de inchamento para $R e=1$ e $R e=0.1$ são apresentados na figura 2.37 onde podemos observar que as simulações com $R e=0.1$ apresentam uma taxa de inchamento maior que as taxas obtidas com $R e=1$.

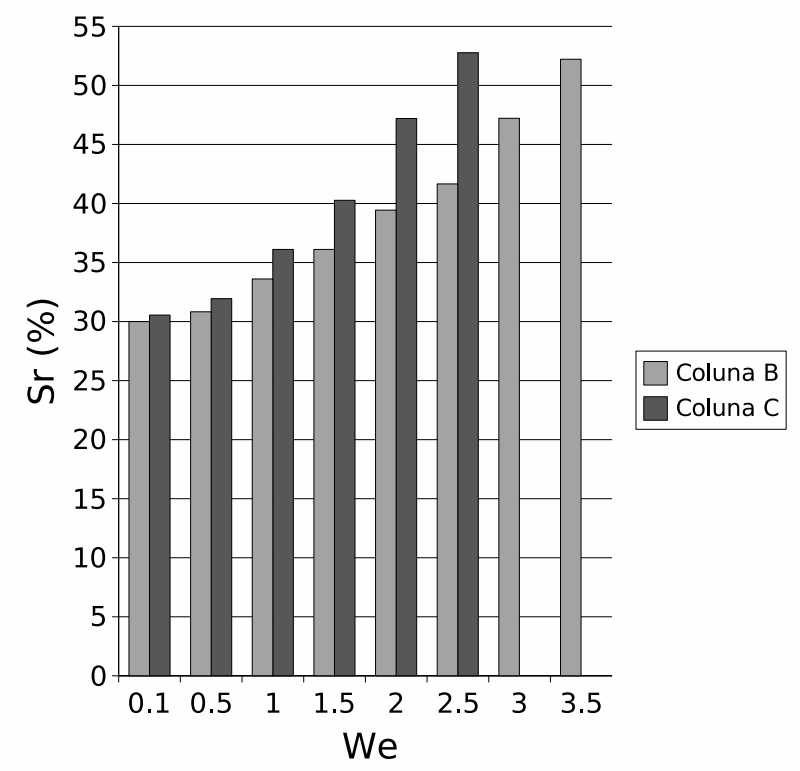

Figura 2.37: Razão de inchamento. Coluna B: $R e=1$. Coluna C: $R e=0.1$ 


\subsubsection{Simulação numérica do impacto de uma gota contra uma su- perfície rígida}

O impacto de uma gota de fluido contra uma superfície rígida é um fato presente em muitas aplicações industriais, tais como, impressão a jato de tinta, pinturas e camadas por spray, etc.. Worthington [90] foi um dos primeiros a investigar impactos sistematicamente e seus livros contêm fascinantes fotografias de fenômenos do impacto de uma esfera sólida e liquida caindo em tanques com liquidos. Estes fenômenos também atraem o público em geral, motivando os consumidores através de comerciais na TV que abusam desses fenômenos, como por exemplo, comerciais onde uma gota de detergente cai sobre uma louça; uma gota de hidrantante ou shampoo cai sobre a palma da mão, entre outros. Há mais de um século estes fenômenos vem sendo estudados e ainda não são compreendidos na sua totalidade e por isso continuam atraindo muitos pesquisadores. Motivados por esses fatos, nesta seção apresentamos resultados numéricos da simulação de uma gota de fluido incidindo perpendicularmente numa superfície plana rígida. Os efeitos que podem ocorrem durante o impacto de uma gota de fluido contra uma superfície plana rígida são conhecidos como 'bouncing', 'spreading' e 'splashing'. Um esboço desses fenômenos é mostrado na figura 2.38 .

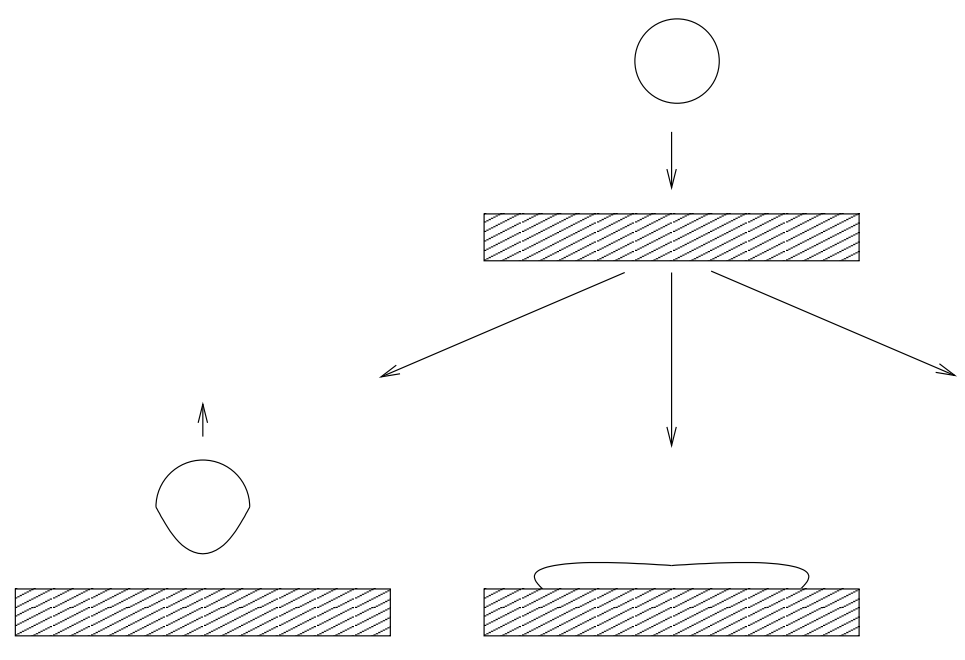

(a)

(b)

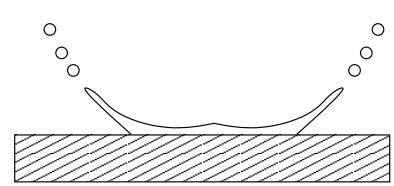

(c)

Figura 2.38: Tipos de fenômenos do impacto de uma gota com fluido contra uma superfície plana rígida: (a) 'bouncing'; (b) 'spreading'; (c) 'splashing'

Para mostrar que o método numérico apresentado nesse trabalho é capaz de simular escoamentos viscoelásticos com superfícies livres, a simulação de uma gota esférica contendo fluido viscoelástico incindindo sobre uma placa rígida é apresentada. Os resultados obtidos são comparados com os resultados obtidos na simulação de uma gota contendo fluido Newtoniano. A geometria adotada nessas simulações é mostrada na figura 2.39 e os dados geométricos utilizados nessas simulações são apresentados na Tabela 2.8. Nas simulações escolhemos $R e=5, W e=2$, 
$\varepsilon=\xi=0.01$. Estas simulações consistem no estudo do comportamento da deformação de duas gotas de fluidos diferentes que ao incidirem contra uma superfície plana rígida sob as mesmas condições apresentam efeitos distintos. Os dados para estas simulações que caracterizam os fluidos estão dispostos na Tabela 2.9. Os resultados dessas simulações são apresentados nas figuras 2.40 e 2.41 em diferentes tempos.

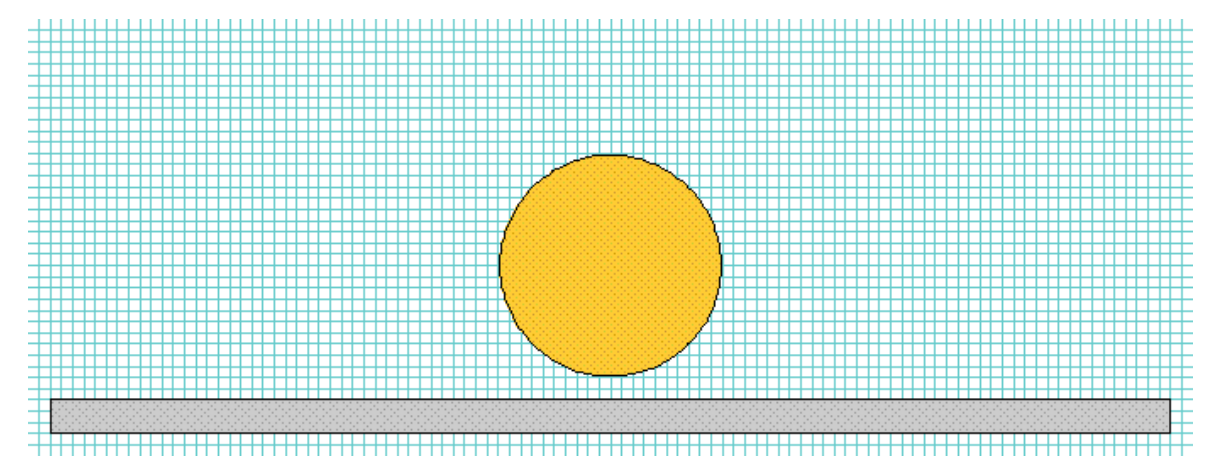

Figura 2.39: Domínio geométrico: $10 \times 3.5 \mathrm{~cm} ; 100 \times 35$ células. Placa rígida de $10 \mathrm{~cm}$. Diâmetro da gota $2 \mathrm{~cm}$. Altura do centro da gota até a placa rígida $1.2 \mathrm{~cm}$

\section{Dados geométricos}

\begin{tabular}{|l|l|}
\hline domínio & $10 \mathrm{~cm} \times 3.5 \mathrm{~cm}$ \\
\hline malha & $100 \times 35$ células \\
\hline altura do centro da gota até a placa rígida & $1.2 \mathrm{~cm}$ \\
\hline diâmetro da gota & $7 \mathrm{~mm}$ \\
\hline
\end{tabular}

Tabela 2.8: Simulação numérica do impacto de uma gota esférica numa placa rígida: Modelagem do problema.

Dados que caracterizam os escoamentos dos fluidos Newtoniano e PTT

\begin{tabular}{|l|l|}
\hline Newtoniano e PTT & $R e=5$ e $W e=2$ \\
\hline \hline$U:$ velocidade inicial da gota & $-2 \mathrm{~ms}^{-1}$ \\
\hline$g_{x}:$ força & $-9.81 \mathrm{~ms}^{-2}$ \\
\hline$\nu=\eta / \rho$ & $0.008 \mathrm{~m}^{2} \mathrm{~s}^{-1}$ \\
\hline$F r$ & 4.515236 \\
\hline modelo PTT & \\
\hline$\lambda$ & $0.02 \mathrm{~s}$ \\
\hline$\varepsilon$ & 0.01 \\
\hline$\xi$ & 0.01 \\
\hline
\end{tabular}

Tabela 2.9: Simulação numérica do impacto de uma gota esférica numa placa rígida: Dados utilizados para simular as gotas de fluido Newtoniano e viscoelástico. 
Podemos observar nas figuras 2.40 e 2.41 que a diferença no comportamento da gota do fluido viscoelástico e da gota do fluido Newtoniano é caracterizada pela mudança na deformação das gotas no decorrer do escoamento. A gota de fluido Newtoniano apresenta o efeito 'spreading' (definido na figura 2.38 (b)), pois há uma redução contínua de velocidade até a gota alcançar sua forma final deformada (ver figura 2.41). A gota contendo o fluido viscoelástico inicialmente apresenta o efeito 'spreading', mas entre os tempos $0.015 \mathrm{~s}$ e $0.030 \mathrm{~s}$, as componentes da velocidade da gota mudam de sinal, indicando um movimento de contração da gota. Portanto, nessa fase a gota viscoelástica apresenta o efeito chamado 'bouncing' (definido na figura 2.38 (a)). A deformação máxima diminui e a gota se aproxima da sua forma deformada final com o decorrer do tempo.

As taxas de deformação e as velocidades diminuem com o passar do tempo até o fluido viscoelástico parar. A figura 2.42 mostra as componentes do tensor não-Newtoniano em diversos tempos, $t=0.002,0.005,0.007,0.009,0.012,0.040,0.342 \mathrm{~s}$. Na figura 2.42 vemos que o comportamento das componentes do tensor $\tau$ revelam a competição existente entre os processos de crescimento/relaxação de tensões. Se houver ainda alguma tensão no fluido, o fluido passa a relaxar essa tensão, aumentando a taxa de deformação e as velocidades, iniciando um novo ciclo de deformação na direção oposta.

A figura 2.43(a) mostra a superfície livre da gota viscoelástica em quatro diferentes tempos do escoamento, $t=0.002,0.005,0.009,0.015 \mathrm{~s}$. Nesta figura pode-se notar uma diminuição na amplitude da gota a cada tempo. Entretanto, na figura 2.43(b) nota-se que há uma leve contração na superfíce lateral da gota aumentando a amplitude local da gota a cada tempo. Os tempos destacados na figura $2.43(\mathrm{~b})$ são $t=0.015,0.020,0.030 \mathrm{~s}$. Na figura $2.43(\mathrm{c})$ nota-se que a amplitude da gota volta a diminuir a cada tempo, ou seja, vimos que do tempo $t=0.20$ para o tempo $t=0.30$ há uma diminuição e já do tempo $t=0.30$ em diante a gota volta a escoar lateralmente diminuindo assim a sua amplitude (ver figura 2.43(d)). Acreditamos que este fenômeno ocorre por causa do aspecto elástico da deformação da gota do fluido viscoelástico.

Para enfatizar o efeito viscoelástico mostrado na figura 2.40, observamos a variação da amplitude da gota viscoelástica no decorrer do escoamento através da figura 2.43. A figura 2.43 mostra a amplitude da gota em diversos tempos. 

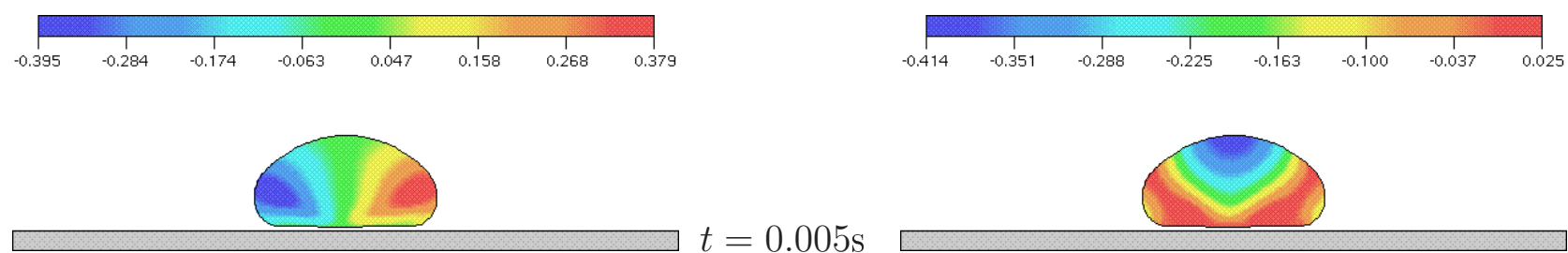

$t=0.005 \mathrm{~s}$
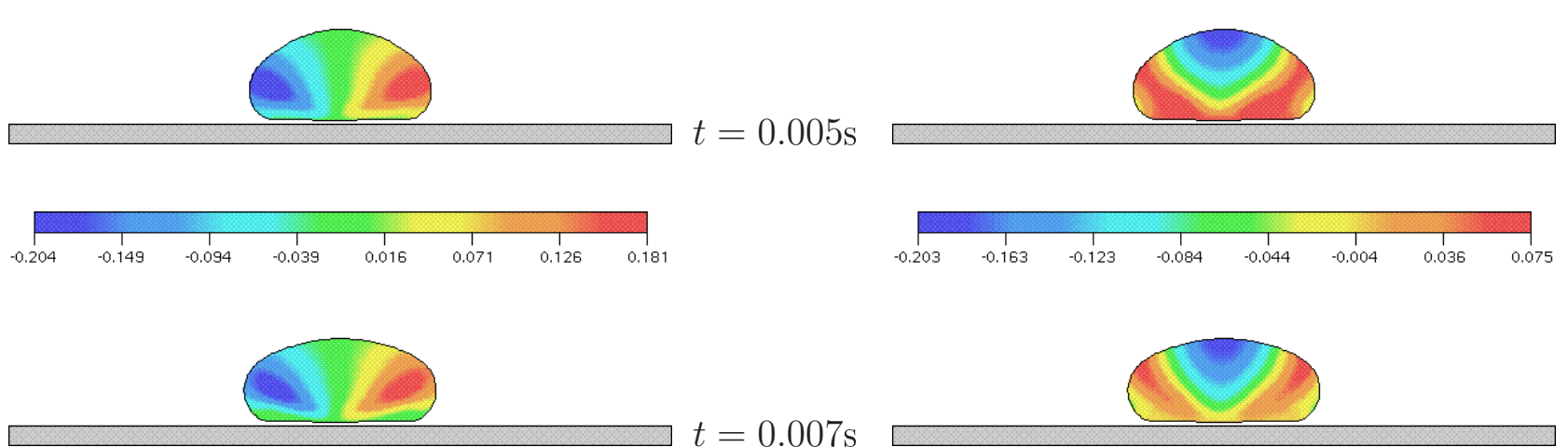

$t=0.007 \mathrm{~s}$
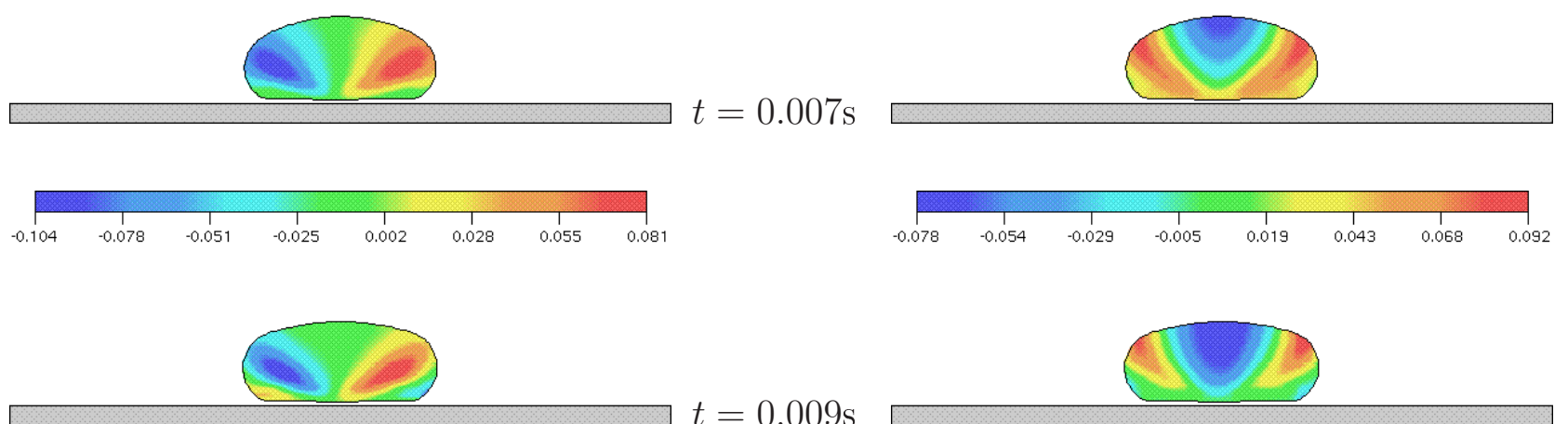

$t=0.009 \mathrm{~s}$
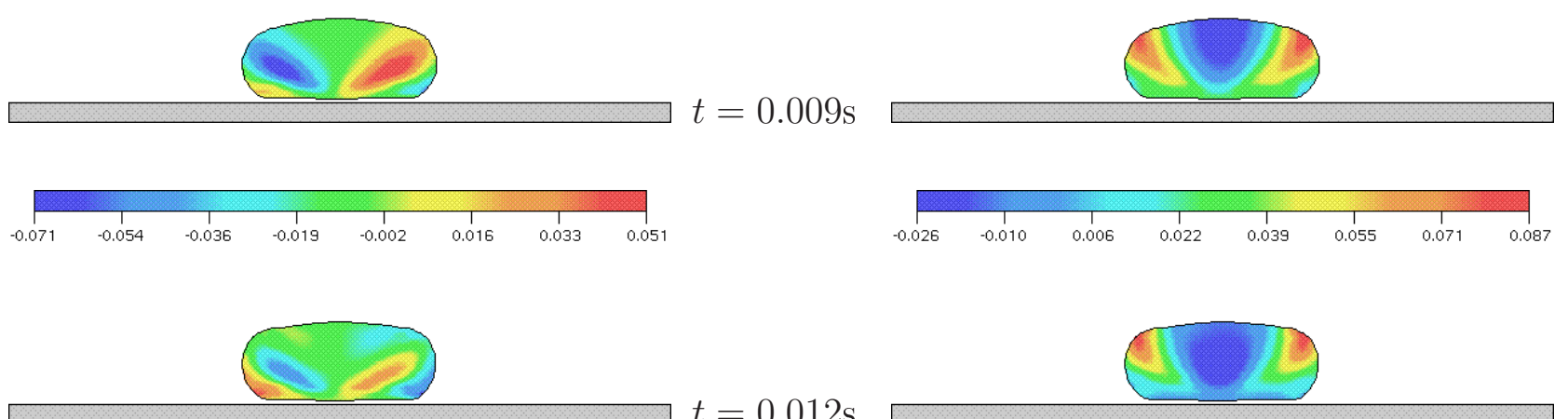

$t=0.012 \mathrm{~s}$
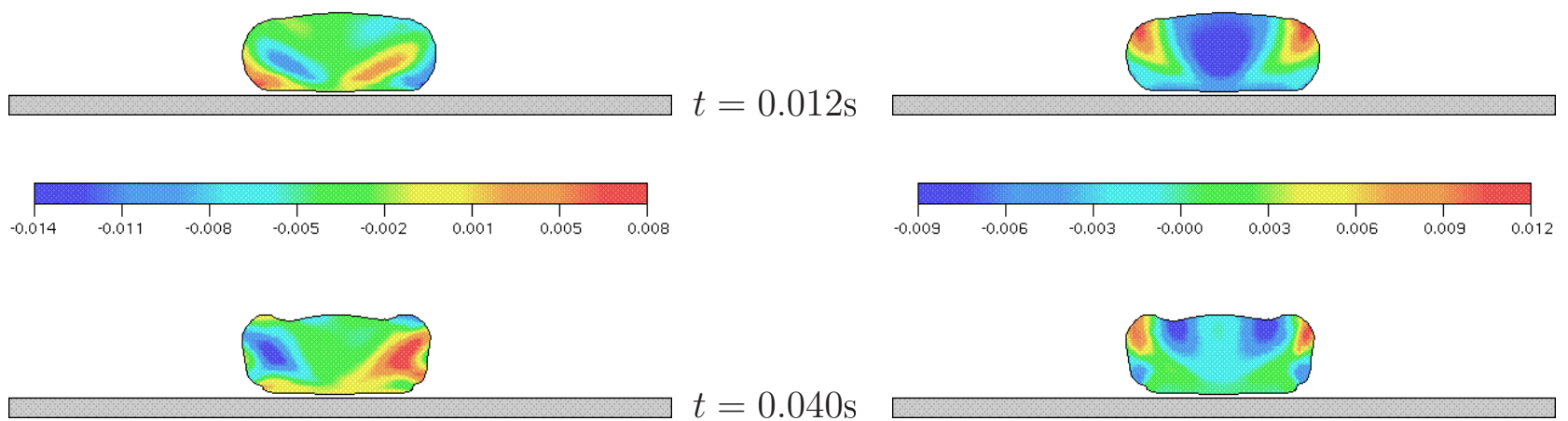

$t=0.040 \mathrm{~s}$

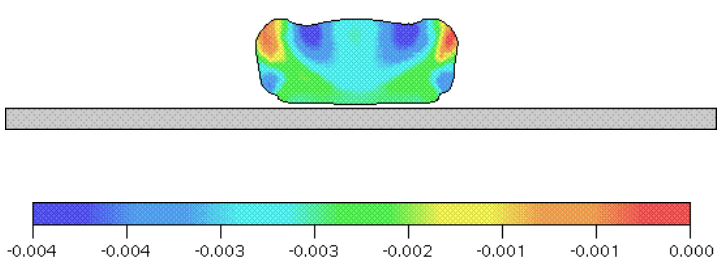

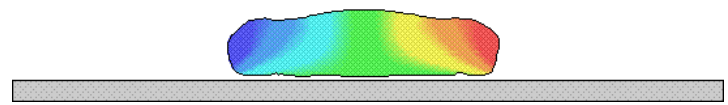

(a) $t=0.342 \mathrm{~s}$

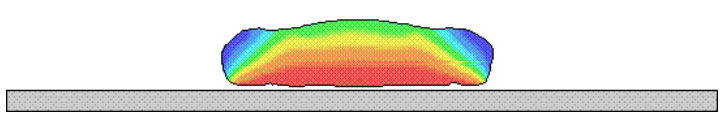

(b)

Figura 2.40: Simulação do impacto de uma gota esférica com fluidoPTT contra uma placa rígida em diferentes tempos. (a) Perfil da componente $u$ de velocidade. (b) Perfil da componente $v$ de velocidade. 

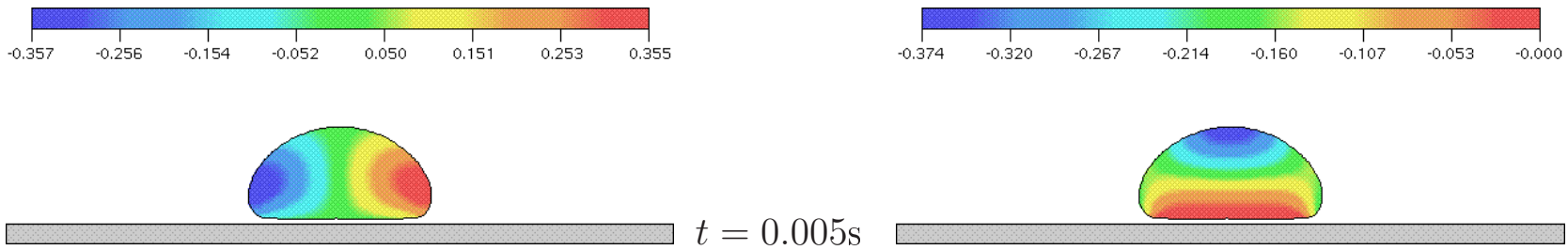

$t=0.005 \mathrm{~s}$
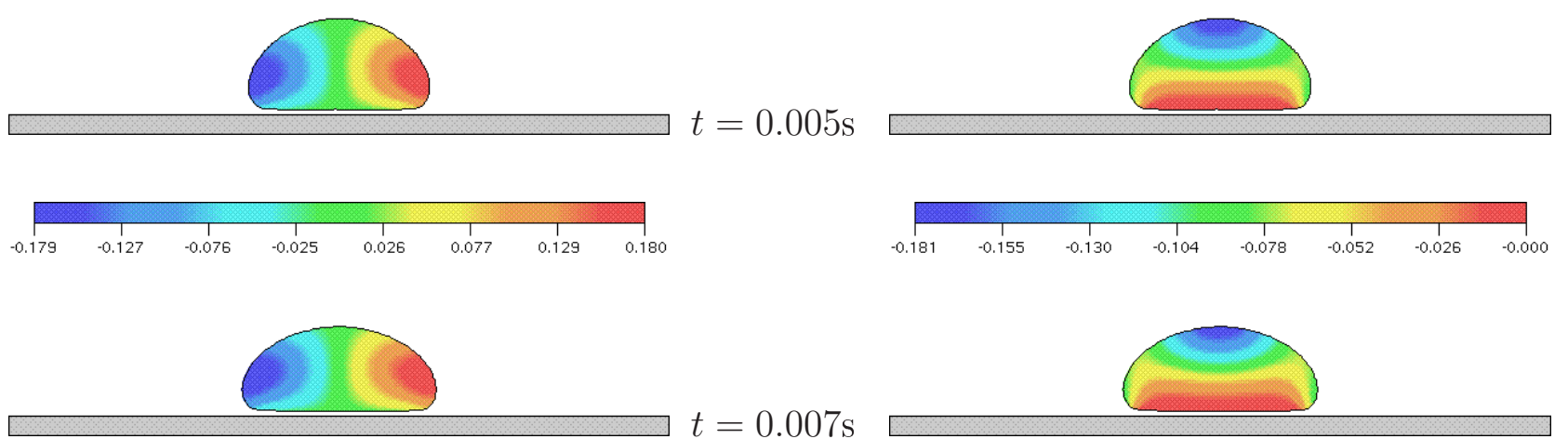

$t=0.007 \mathrm{~s}$
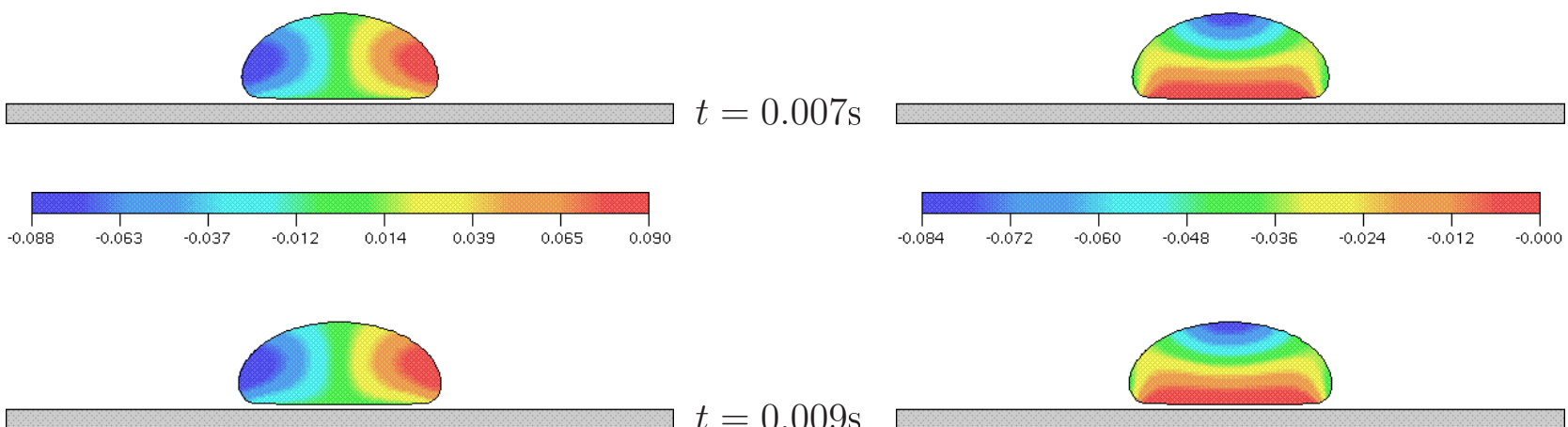

$t=0.009 \mathrm{~s}$
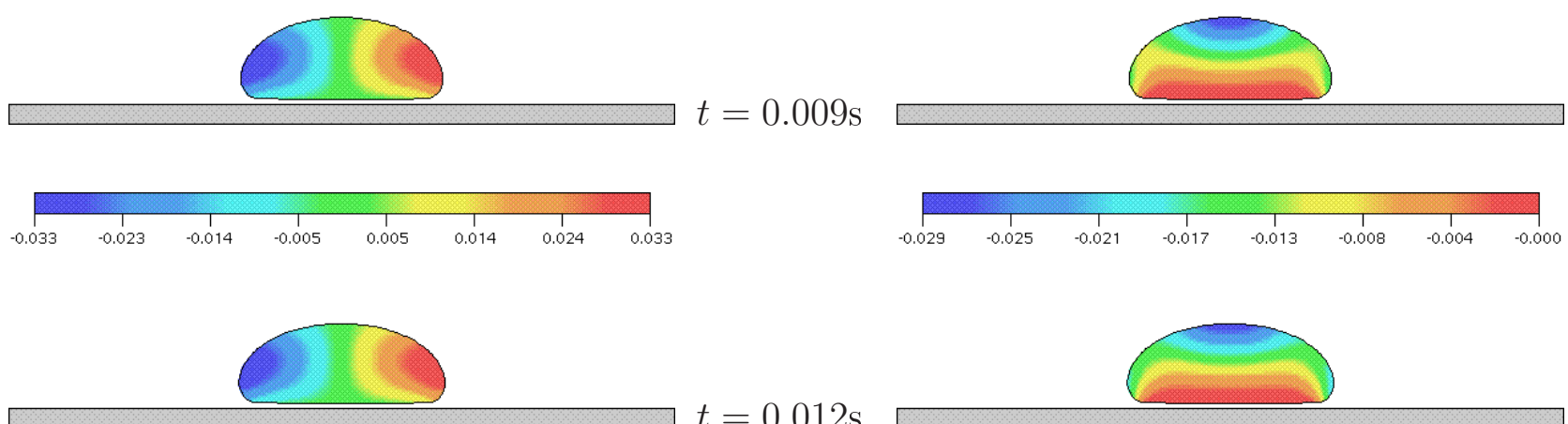

$t=0.012 \mathrm{~s}$
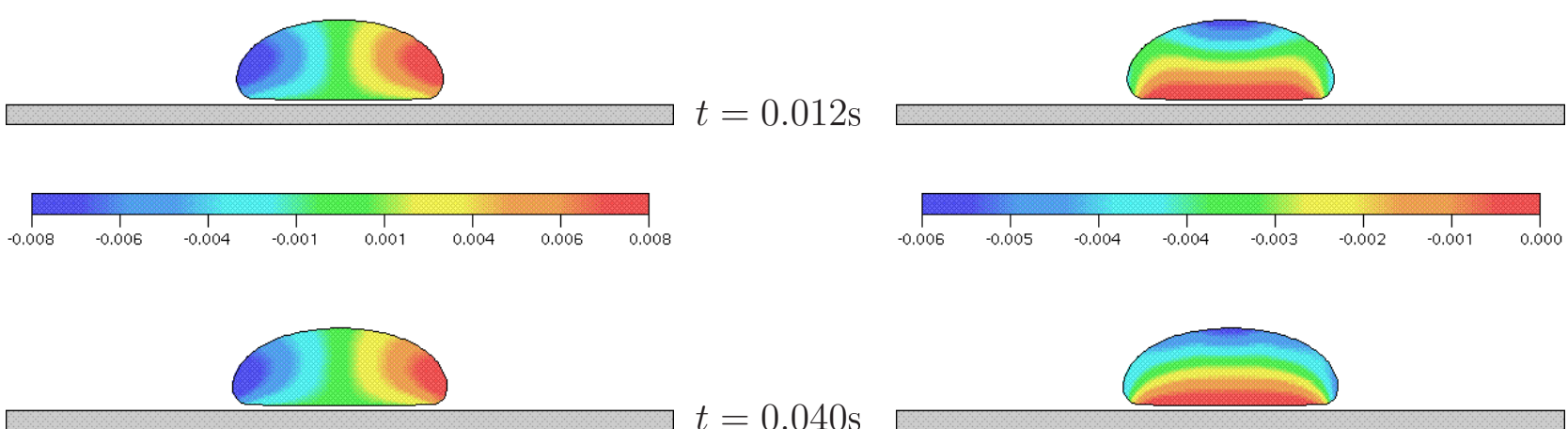

$t=0.040 \mathrm{~s}$
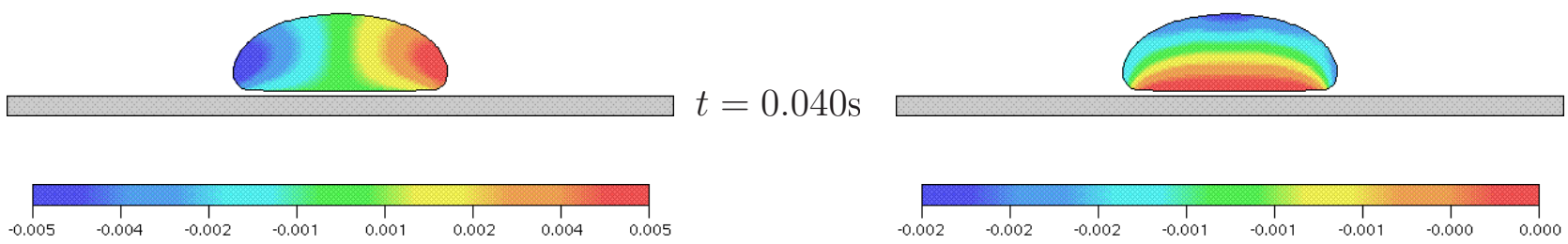

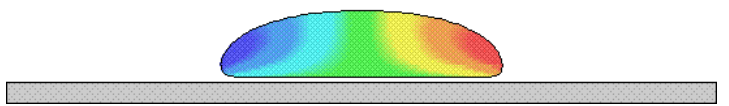

(a) $t=0.342 \mathrm{~s}$

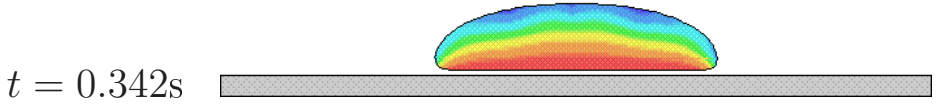

(b)

Figura 2.41: Simulação do impacto de uma gota esférica de fluido Newtoniano contra uma placa rígida em diferentes tempos. (a) Perfil da componente $u$ de velocidade. (b) Perfil da componente $v$ de velocidade. 


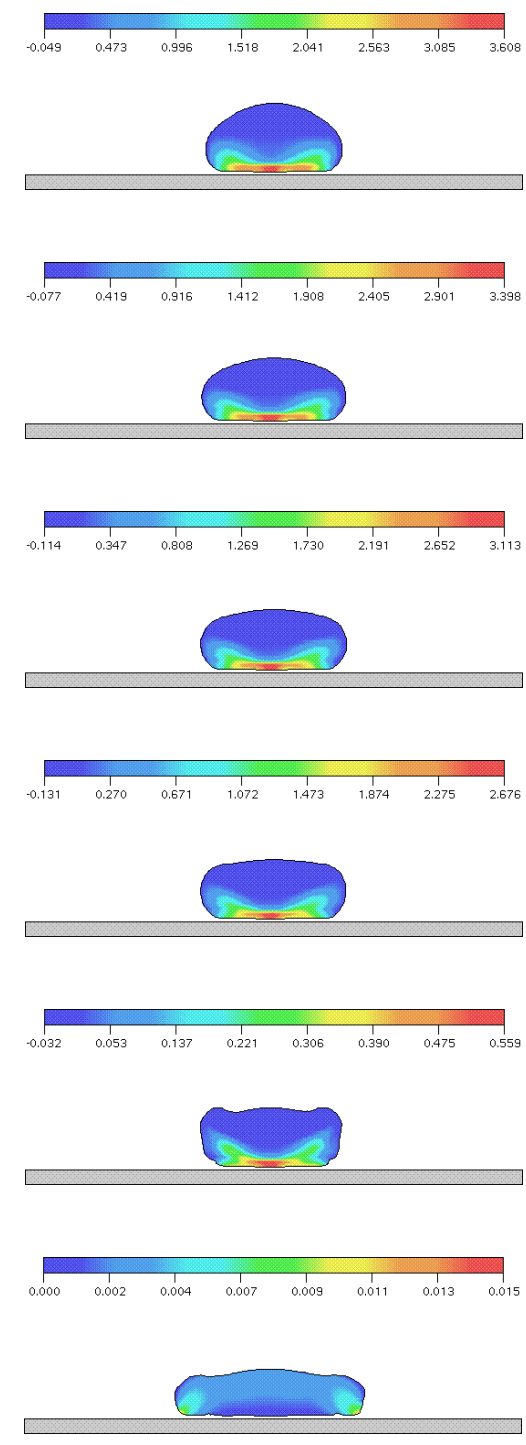

(a)
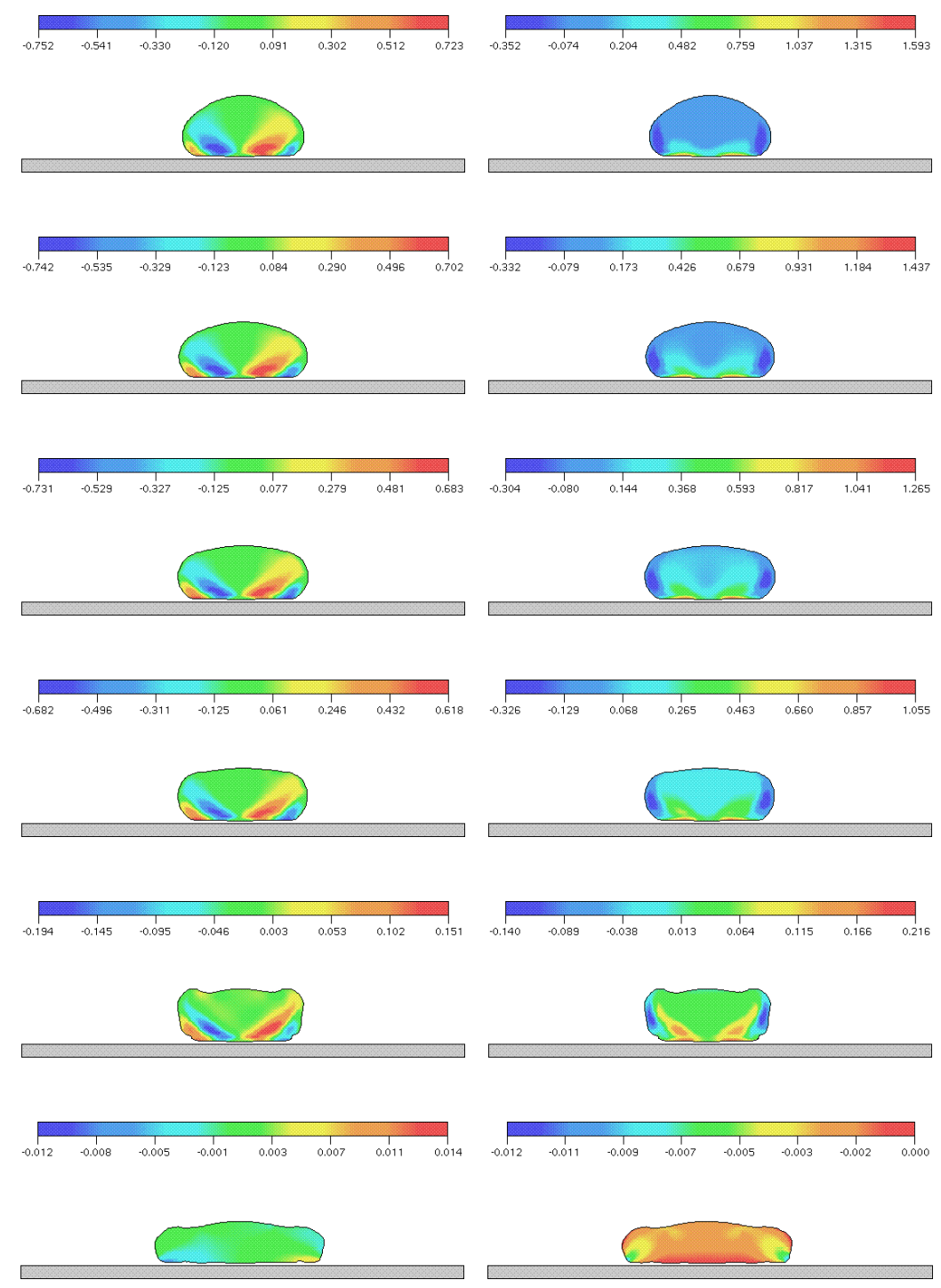

(b)

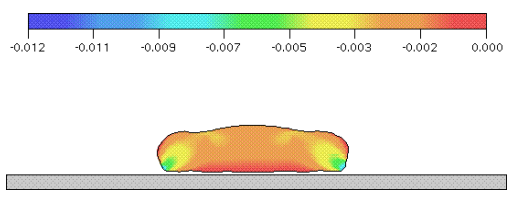

(c)

Figura 2.42: Modelo PTT: simulação do impacto de uma gota esférica de fluido viscoelástico contra uma placa rígida em diferentes tempos. (a) Perfil da componete $\tau^{x x}$ do tensor. (b) Perfil da componente $\tau^{x y}$ do tensor. (c) Perfil da componente $\tau^{y y}$ do tensor. 


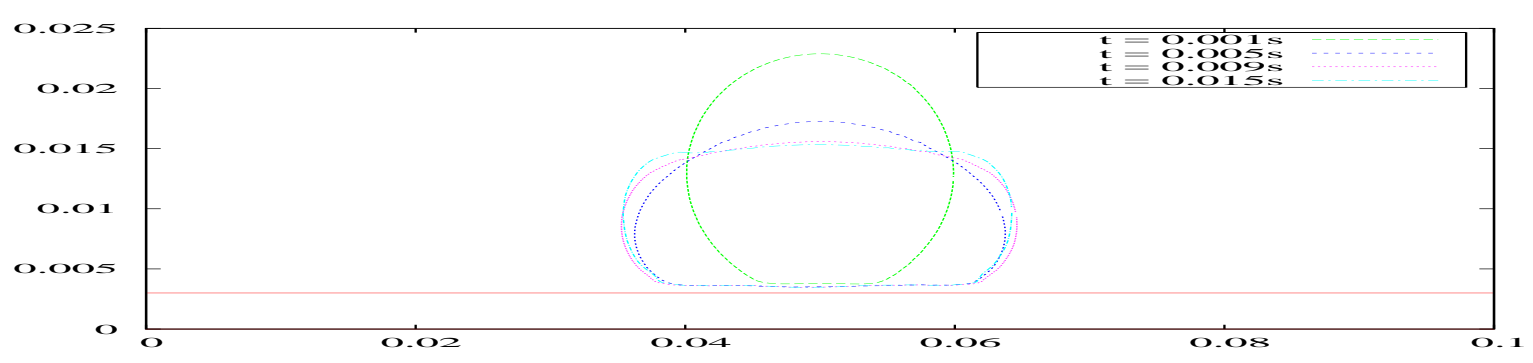

(a)

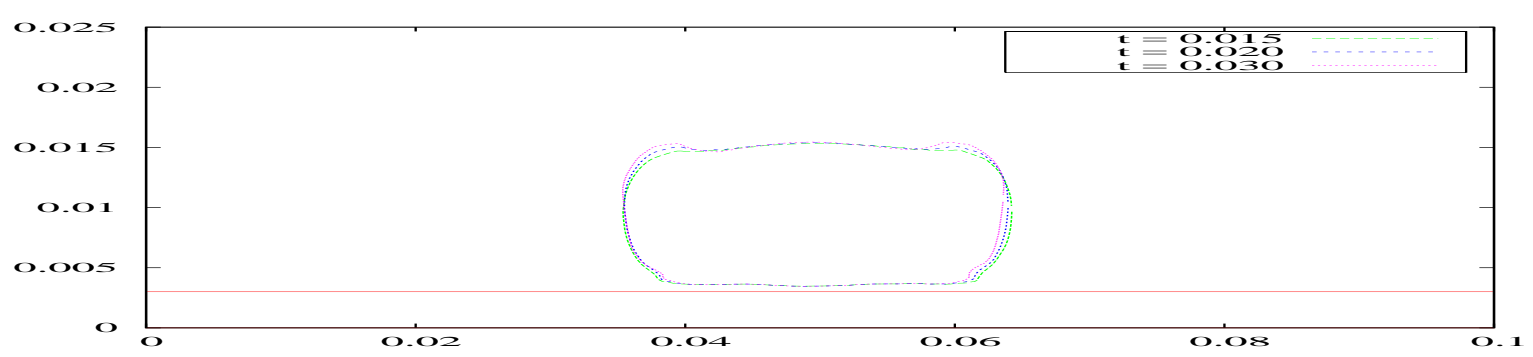

(b)

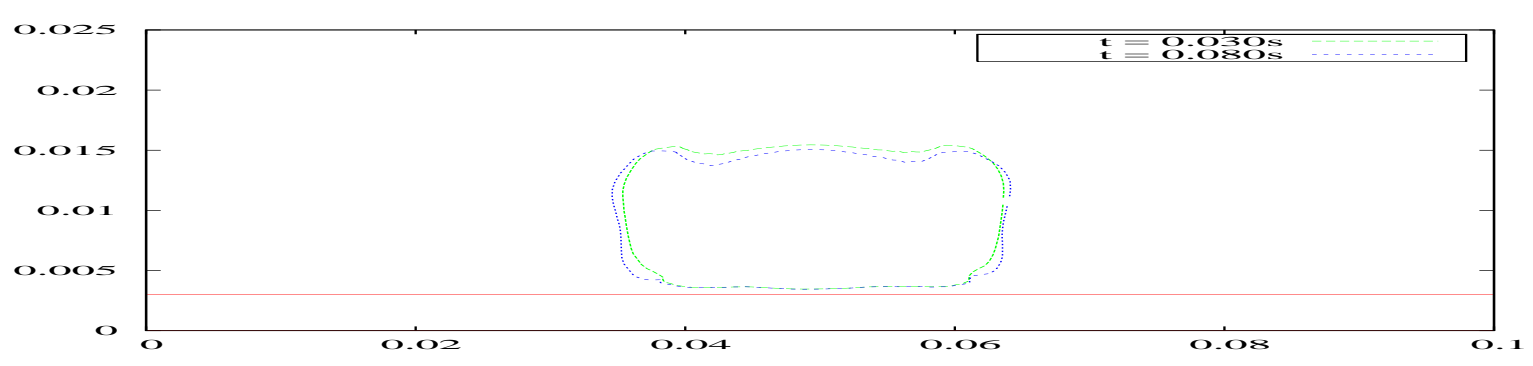

(c)

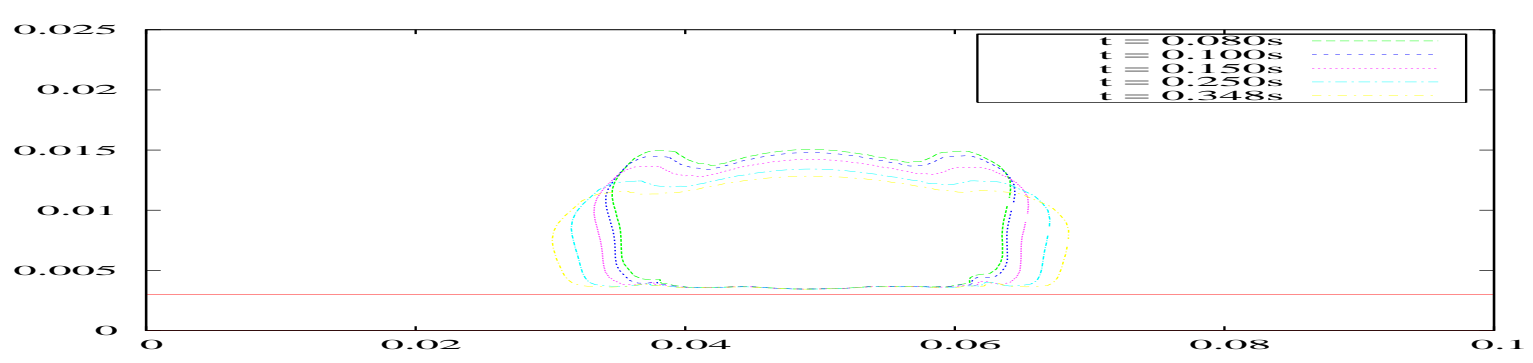

(d)

Figura 2.43: Gráficos da superfície livre da gota viscoelástica em diversos tempos da simulação. (a) $t=0.002,0.005,0.009,0.015$ s. (b) $t=0.015,0.020,0.030 \mathrm{~s} . \quad(\mathrm{c}) t=0.030,0.080 \mathrm{~s}$. (d) $t=0.080,0.100,0.150,0.250,0.348$ s. $R e=5, W e=2, \xi=0.01$ e $\varepsilon=0.01$. 


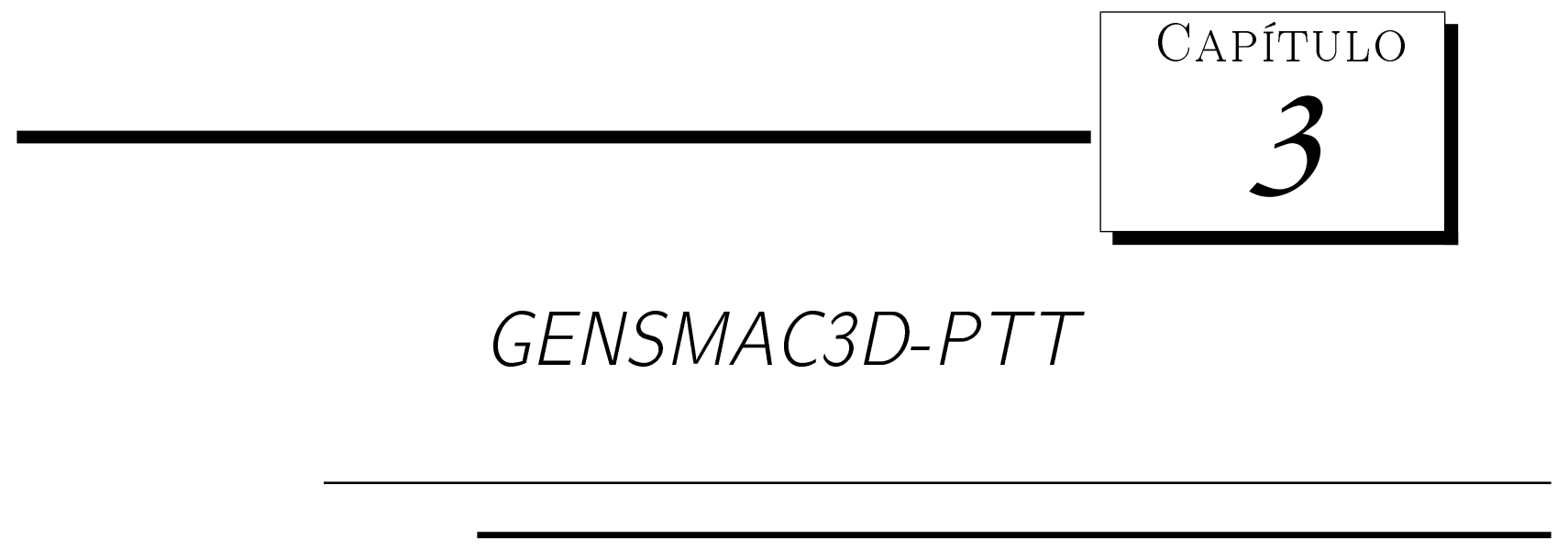

Nesse capítulo vamos apresentar um método numérico para simular escoamentos viscoelásticos tridimensionais governados pela equação constitutiva PTT. O método numérico proposto é uma extensão da técnica apresentada no capítulo 2, para simular escoamentos bidimensionais, para escoamentos tridimensionais. As equações básicas que governam escoamentos incompressíveis tridimensionais, isotérmicos, descritos pelo modelo PTT são apresentadas. O tratamento do tensor não-Newtoniano em contorno rígidos tridimensionais é apresentado em detalhes bem como o cálculo das condições de contorno na superfície livre. As equações governantes são resolvidas pelo método de diferenças finitas utilizando uma malha deslocada tridimensional. O fluido é modelado pelo método Marker-and-Cell e tratado como uma superfície linear por partes. O método desenvolvido nesse trabalho foi implementado no sistema FreeFlow3D e resultados numéricos obtidos na simulação de escoamentos tridimensionais governados pela equação constitutiva PTT são apresentados. Os resultados numéricos incluem: simulação do jato oscilante tridimensional e inchamento do extrudado.

\subsection{Formulação do problema tridimensional}

As equações que governam escoamentos incompressíveis governados pelo modelo PTT são a equação de conservação de massa, equação de conservação de quantidade de movimento e a equação constitutiva PTT que são dadas por (conforme visto no capítulo 3, as quais repetimos aqui por conveniência):

$$
\begin{gathered}
\frac{\partial u_{i}}{\partial x_{i}}=0 \\
\rho \frac{\partial u_{i}}{\partial t}+\rho \frac{\partial u_{k} u_{i}}{\partial x_{k}}=-\frac{\partial p}{\partial x_{i}}+\eta \frac{\partial}{\partial x_{k}}\left(\frac{\partial u_{i}}{\partial x_{k}}\right)+\frac{\partial S_{i k}}{\partial x_{k}}+\rho g_{i}
\end{gathered}
$$




$$
f\left(S_{k k}\right) S_{i j}+\lambda \stackrel{\square}{S}_{i j}=2 \eta\left[1-f\left(S_{k k}\right)\right] D_{i j}-2 \lambda \eta \stackrel{\square}{D}_{i j}
$$

onde o tensor das tensões é dado por

$$
\tau_{i j}=2 \eta D_{i j}+S_{i j}
$$

em que $2 \eta D_{i j}$ é o tensor de contribuição Newtoniana, $S_{i j}$ é tensor de contribuição não-Newtoniana.

Portanto, devemos resolver as equações $(3.1),(3.2)$ e (3.3) para as variáveis $p, u_{i}$ e $S_{i j}$.

\subsection{Adimensionalização}

Nesse trabalho vamos adotar escoamentos tridimensionais cartesianos com $x_{i}=(x, y, z)$. As componentes do vetor velocidade $u_{i}$ nas direções $x, y$ e $z$ serão denominadas $u, v$ e $w$, respectivamente. O tensor não-Newtoniano $S_{i j}$ será representado por:

$$
S_{i j}=\left[\begin{array}{ccc}
S^{x x} & S^{x y} & S^{x z} \\
S^{x y} & S^{y y} & S^{y z} \\
S^{x z} & S^{y z} & S^{z z}
\end{array}\right]
$$

Com objetivo de simplificar a notação, as vezes denotaremos apenas $u, v$ e $w$ ao invés de $u(x, y, t), v(x, y, t), w(x, y, t)$.

Os problemas de mecânica dos fluidos, em geral, são caracterizados por grandezas específicas, como velocidade, viscosidade, massa específica do escoamento e também o diamêtro de um tubo, por exemplo. Essas grandezas dimensionais podem ser agrupadas em parâmetros adimensionais, que passam a caracterizar o escoamento. Neste trabalho, usaremos os seguintes números admensionais: Reynolds, Weissenberg e Froude. Para a adimensionalização das equações, as unidades escalares de comprimento, velocidade, viscosidade e densidade serão denotadas por $L$, $U, \eta$ e $\rho$, respectivamente. A adimensionalização pode ser feita a partir das seguintes grandezas adimensionais:

$$
\begin{aligned}
& x=L \bar{x}, \quad u=U \bar{u}, \quad S^{x x}=\rho U^{2} \bar{S}^{x x}, \quad S^{x y}=\rho U^{2} \bar{S}^{x y}, \quad g_{x}=g \bar{g}_{x}, \\
& y=L \bar{y}, \quad v=U \bar{v}, \quad S^{y y}=\rho U^{2} \bar{S}^{y y}, \quad S^{x z}=\rho U^{2} \bar{S}^{x z}, \quad g_{y}=g \bar{g}_{y}, \\
& z=L \bar{z}, \quad w=U \bar{w}, \quad S^{z z}=\rho U^{2} \bar{S}^{z z}, \quad S^{y z}=\rho U^{2} \bar{S}^{y z}, \quad g_{z}=g \bar{g}_{z}, \\
& t=(L / U) \bar{t}, \quad p=\rho U^{2} \bar{p},
\end{aligned}
$$

Introduzindo essa adimensionalização nas equações (3.1), (3.2) e (3.3) e utilizando coordenadas cartesianas, obtemos as equações adimensionais (as barras foram omitidas por conveniência):

$$
\frac{\partial u}{\partial x}+\frac{\partial v}{\partial y}+\frac{\partial w}{\partial z}=0
$$




$$
\begin{aligned}
\frac{\partial u}{\partial t}= & -\frac{\partial u^{2}}{\partial x}-\frac{\partial(v u)}{\partial y}-\frac{\partial(w u)}{\partial z}-\frac{\partial p}{\partial x}+\frac{1}{R e}\left(\frac{\partial^{2} u}{\partial x^{2}}+\frac{\partial^{2} u}{\partial y^{2}}+\frac{\partial^{2} u}{\partial z^{2}}\right)+\frac{\partial S^{x x}}{\partial x}+\frac{\partial S^{x y}}{\partial y} \\
& +\frac{\partial S^{x z}}{\partial z}+\frac{1}{F r^{2}} g_{x}, \\
\frac{\partial v}{\partial t}= & -\frac{\partial(u v)}{\partial x}-\frac{\partial v^{2}}{\partial y}-\frac{\partial(w v)}{\partial z}-\frac{\partial p}{\partial y}+\frac{1}{R e}\left(\frac{\partial^{2} v}{\partial x^{2}}+\frac{\partial^{2} v}{\partial y^{2}}+\frac{\partial^{2} v}{\partial z^{2}}\right)+\frac{\partial S^{x y}}{\partial x}+\frac{\partial S^{y y}}{\partial y} \\
& +\frac{\partial S^{y z}}{\partial z}+\frac{1}{F r^{2}} g_{y}, \\
\frac{\partial w}{\partial t}= & -\frac{\partial(u w)}{\partial x}-\frac{\partial v w}{\partial y}-\frac{\partial w^{2}}{\partial z}-\frac{\partial p}{\partial z}+\frac{1}{R e}\left(\frac{\partial^{2} w}{\partial x^{2}}+\frac{\partial^{2} w}{\partial y^{2}}+\frac{\partial^{2} w}{\partial z^{2}}\right)+\frac{\partial S^{x z}}{\partial x}+\frac{\partial S^{y z}}{\partial y} \\
& +\frac{\partial S^{z z}}{\partial z}+\frac{1}{F r^{2}} g_{z},
\end{aligned}
$$

$$
\frac{\partial S^{x x}}{\partial t}=-f\left(S_{k k}\right) \frac{1}{W e} S^{x x}-\frac{\partial\left(u S^{x x}\right)}{\partial x}-\frac{\partial\left(v S^{x x}\right)}{\partial y}-\frac{\partial\left(w S^{x x}\right)}{\partial z}+2(1-\xi) D^{x x} S^{x x}
$$$$
+\left[(2-\xi) \frac{\partial u}{\partial y}-\xi \frac{\partial v}{\partial x}\right] S^{x y}+\left[(2-\xi) \frac{\partial u}{\partial z}-\xi \frac{\partial w}{\partial x}\right] S^{x z}+\frac{2}{R e W e}\left[1-f\left(S_{k k}\right)\right] D^{x x}
$$$$
-\frac{2}{R e}\left\{\frac{\partial D^{x x}}{\partial t}+\frac{\partial\left(u D^{x x}\right)}{\partial x}+\frac{\partial\left(v D^{x x}\right)}{\partial y}+\frac{\partial\left(w D^{x x}\right)}{\partial z}-2(1-\xi)\left(D^{x x}\right)^{2}\right.
$$

$$
\begin{aligned}
& \left.-\left[(2-\xi) \frac{\partial u}{\partial y}-\xi \frac{\partial v}{\partial x}\right] D^{x y}-\left[(2-\xi) \frac{\partial u}{\partial z}-\xi \frac{\partial w}{\partial x}\right] D^{x z}\right\}, \\
\frac{\partial S^{y y}}{\partial t} & =-f\left(S_{k k}\right) \frac{1}{W e} S^{y y}-\frac{\partial\left(u S^{y y}\right)}{\partial x}-\frac{\partial\left(v S^{y y}\right)}{\partial y}-\frac{\partial\left(w S^{y y}\right)}{\partial z}+2(1-\xi) D^{y y} S^{y y}
\end{aligned}
$$$$
+\left[(2-\xi) \frac{\partial v}{\partial x}-\xi \frac{\partial u}{\partial y}\right] S^{x y}+\left[(2-\xi) \frac{\partial v}{\partial z}-\xi \frac{\partial w}{\partial y}\right] S^{y z}+\frac{2}{R e W e}\left[1-f\left(S_{k k}\right)\right] D^{y y}
$$$$
-\frac{2}{R e}\left\{\frac{\partial D^{y y}}{\partial t}+\frac{\partial\left(u D^{y y}\right)}{\partial x}+\frac{\partial\left(v D^{y y}\right)}{\partial y}+\frac{\partial\left(w D^{y y}\right)}{\partial z}-2(1-\xi)\left(D^{y y}\right)^{2}\right.
$$$$
\left.-\left[(2-\xi) \frac{\partial v}{\partial x}-\xi \frac{\partial u}{\partial y}\right] D^{x y}-\left[(2-\xi) \frac{\partial v}{\partial z}-\xi \frac{\partial w}{\partial y}\right] D^{y z}\right\}
$$

$$
\begin{aligned}
\frac{\partial S^{z z}}{\partial t} & =-f\left(S_{k k}\right) \frac{1}{W e} S^{z z}-\frac{\partial\left(u S^{z z}\right)}{\partial x}-\frac{\partial\left(v S^{z z}\right)}{\partial y}-\frac{\partial\left(w S^{z z}\right)}{\partial z}+2(1-\xi) D^{z z} S^{z z} \\
& +\left[(2-\xi) \frac{\partial w}{\partial x}-\xi \frac{\partial u}{\partial z}\right] S^{x z}+\left[(2-\xi) \frac{\partial w}{\partial y}-\xi \frac{\partial v}{\partial z}\right] S^{y z}+\frac{2}{\operatorname{ReWe}}\left[1-f\left(S_{k k}\right)\right] D^{z z} \\
& -\frac{2}{R e}\left\{\frac{\partial D^{z z}}{\partial t}+\frac{\partial\left(u D^{z z}\right)}{\partial x}+\frac{\partial\left(v D^{z z}\right)}{\partial y}+\frac{\partial\left(w D^{z z}\right)}{\partial z}-2(1-\xi)\left(D^{z z}\right)^{2}\right. \\
& \left.-\left[(2-\xi) \frac{\partial w}{\partial x}-\xi \frac{\partial u}{\partial z}\right] D^{x z}-\left[(2-\xi) \frac{\partial w}{\partial y}-\xi \frac{\partial v}{\partial z}\right] D^{y z}\right\}
\end{aligned}
$$




$$
\begin{aligned}
\frac{\partial S^{x y}}{\partial t} & =-f\left(S_{k k}\right) \frac{1}{W e} S^{x y}-\frac{\partial\left(u S^{x y}\right)}{\partial x}-\frac{\partial\left(v S^{x y}\right)}{\partial y}-\frac{\partial\left(w S^{x y}\right)}{\partial z}+(1-\xi)\left(D^{x x}+D^{y y}\right) S^{x y} \\
& +\left[\left(1-\frac{\xi}{2}\right) \frac{\partial v}{\partial x}-\frac{\xi}{2} \frac{\partial u}{\partial y}\right] S^{x x}+\left[\left(1-\frac{\xi}{2}\right) \frac{\partial u}{\partial y}-\frac{\xi}{2} \frac{\partial v}{\partial x}\right] S^{y y} \\
& +\left[\left(1-\frac{\xi}{2}\right) \frac{\partial v}{\partial z}-\frac{\xi}{2} \frac{\partial w}{\partial y}\right] S^{x z}+\left[\left(1-\frac{\xi}{2}\right) \frac{\partial u}{\partial z}-\frac{\xi}{2} \frac{\partial w}{\partial x}\right] S^{y z}+\frac{2}{R e W e}\left[1-f\left(S_{k k}\right)\right] D^{x y} \\
& -\frac{2}{R e}\left\{\frac{\partial D^{x y}}{\partial t}+\frac{\partial\left(u D^{x y}\right)}{\partial x}+\frac{\partial\left(v D^{x y}\right)}{\partial y}+\frac{\partial\left(w D^{x y}\right)}{\partial z}-(1-\xi)\left(D^{x x}+D^{y y}\right) D^{x y}\right. \\
& -\left[\left(1-\frac{\xi}{2}\right) \frac{\partial v}{\partial x}-\frac{\xi}{2} \frac{\partial u}{\partial y}\right] D^{x x}-\left[\left(1-\frac{\xi}{2}\right) \frac{\partial u}{\partial y}-\frac{\xi}{2} \frac{\partial v}{\partial x}\right] D^{y y} \\
& \left.-\left[\left(1-\frac{\xi}{2}\right) \frac{\partial v}{\partial z}-\frac{\xi}{2} \frac{\partial w}{\partial y}\right] D^{x z}-\left[\left(1-\frac{\xi}{2}\right) \frac{\partial u}{\partial z}-\frac{\xi}{2} \frac{\partial w}{\partial x}\right] D^{y z}\right\}
\end{aligned}
$$

$$
\begin{aligned}
\frac{\partial S^{x z}}{\partial t} & =-f\left(S_{k k}\right) \frac{1}{W e} S^{x z}-\frac{\partial\left(u S^{x z}\right)}{\partial x}-\frac{\partial\left(v S^{x z}\right)}{\partial y}-\frac{\partial\left(w S^{x z}\right)}{\partial z}+(1-\xi)\left(D^{x x}+D^{z z}\right) S^{x z} \\
+ & {\left[\left(1-\frac{\xi}{2}\right) \frac{\partial w}{\partial x}-\frac{\xi}{2} \frac{\partial u}{\partial z}\right] S^{x x}+\left[\left(1-\frac{\xi}{2}\right) \frac{\partial u}{\partial z}-\frac{\xi}{2} \frac{\partial w}{\partial x}\right] S^{z z} } \\
+ & {\left[\left(1-\frac{\xi}{2}\right) \frac{\partial w}{\partial y}-\frac{\xi}{2} \frac{\partial v}{\partial z}\right] S^{x y}+\left[\left(1-\frac{\xi}{2}\right) \frac{\partial u}{\partial y}-\frac{\xi}{2} \frac{\partial v}{\partial x}\right] S^{y z}+\frac{2}{R e W e}\left[1-f\left(S_{k k}\right)\right] D^{x z} } \\
& -\frac{2}{R e}\left\{\frac{\partial D^{x z}}{\partial t}+\frac{\partial\left(u D^{x z}\right)}{\partial x}+\frac{\partial\left(v D^{x z}\right)}{\partial y}+\frac{\partial\left(w D^{x z}\right)}{\partial z}-(1-\xi)\left(D^{x x}+D^{z z}\right) D^{x z}\right. \\
& -\left[\left(1-\frac{\xi}{2}\right) \frac{\partial w}{\partial x}-\frac{\xi}{2} \frac{\partial u}{\partial z}\right] D^{x x}-\left[\left(1-\frac{\xi}{2}\right) \frac{\partial u}{\partial z}-\frac{\xi}{2} \frac{\partial w}{\partial x}\right] D^{z z} \\
& \left.-\left[\left(1-\frac{\xi}{2}\right) \frac{\partial w}{\partial y}-\frac{\xi}{2} \frac{\partial v}{\partial z}\right] D^{x y}-\left[\left(1-\frac{\xi}{2}\right) \frac{\partial u}{\partial y}-\frac{\xi}{2} \frac{\partial v}{\partial x}\right] D^{y z}\right\}
\end{aligned}
$$

$$
\begin{aligned}
\frac{\partial S^{y z}}{\partial t} & =-f\left(S_{k k}\right) \frac{1}{W e} S^{y z}-\frac{\partial\left(u S^{y z}\right)}{\partial x}-\frac{\partial\left(v S^{y z}\right)}{\partial y}-\frac{\partial\left(w S^{y z}\right)}{\partial z}+(1-\xi)\left(D^{y y}+D^{z z}\right) S^{y z} \\
& +\left[\left(1-\frac{\xi}{2}\right) \frac{\partial w}{\partial y}-\frac{\xi}{2} \frac{\partial v}{\partial z}\right] S^{y y}+\left[\left(1-\frac{\xi}{2}\right) \frac{\partial v}{\partial z}-\frac{\xi}{2} \frac{\partial w}{\partial y}\right] S^{z z} \\
& +\left[\left(1-\frac{\xi}{2}\right) \frac{\partial w}{\partial x}-\frac{\xi}{2} \frac{\partial u}{\partial z}\right] S^{x y}+\left[\left(1-\frac{\xi}{2}\right) \frac{\partial v}{\partial x}-\frac{\xi}{2} \frac{\partial u}{\partial y}\right] S^{x z}+\frac{2}{R e W e}\left[1-f\left(S_{k k}\right)\right] D^{y z} \\
& -\frac{2}{R e}\left\{\frac{\partial D^{y z}}{\partial t}+\frac{\partial\left(u D^{y z}\right)}{\partial x}+\frac{\partial\left(v D^{y z}\right)}{\partial y}+\frac{\partial\left(w D^{y z}\right)}{\partial z}-(1-\xi)\left(D^{y y}+D^{z z}\right) D^{y z}\right. \\
& -\left[\left(1-\frac{\xi}{2}\right) \frac{\partial w}{\partial y}-\frac{\xi}{2} \frac{\partial v}{\partial z}\right] D^{y y}-\left[\left(1-\frac{\xi}{2}\right) \frac{\partial v}{\partial z}-\frac{\xi}{2} \frac{\partial w}{\partial y}\right] D^{z z} \\
& \left.-\left[\left(1-\frac{\xi}{2}\right) \frac{\partial w}{\partial x}-\frac{\xi}{2} \frac{\partial u}{\partial z}\right] D^{x y}-\left[\left(1-\frac{\xi}{2}\right) \frac{\partial v}{\partial x}-\frac{\xi}{2} \frac{\partial u}{\partial y}\right] D^{x z}\right\}
\end{aligned}
$$

onde $f\left(S_{k k}\right)=1+\operatorname{Re} W e \varepsilon\left(S_{k k}\right) ; \operatorname{Re}=\rho U L / \eta, W e=\lambda U / L$ e $F r=U / \sqrt{L|g|}$ denotam os números de Reynolds, de Weissenberg e de Froude, respectivamente. 


\subsection{Condições de contorno}

Para resolver as equações (3.6)-(3.14) é necessário impor condições fisicamente adequadas para que o problema, representado por estas equações, possua solução. Essas condições são chamadas de condições iniciais e de contorno. As condições iniciais do escoamento para as componentes de $u_{i}, \tau_{i j}$ e $S_{i j}$ foram adotas como sendo nulas:

$$
u_{i}=0, \quad \tau_{i j}=0 \quad \text { e } \quad S_{i j}=0, \quad i, j=1,2,3
$$

Neste trabalho devemos considerar condições de contorno para as variáveis dependentes nos seguintes tipos de contornos:

- contorno rígido: representa uma parede rígida do domínio do escoamento, como por exemplo a parede container, um tubo, uma placa rígida, etc.;

- injetor: é a região do domínio do escoamento onde há entrada de fluido;

- ejetor: é a região do domínio do escoamento onde há saída de fluido;

- superfície livre: é a interface entre o fluido e a atmosfera.

Detalhes sobre cada condição de contorno nos contornos citados acima são dados a seguir.

\subsubsection{Contornos de injeção e ejeção}

Nesses contornos, a velocidade e as componentes do tensor não-Newtoniano são obtidos como segue:

- Contornos de injeção

No injetor assume-se que as componentes da velocidade são dadas por

$$
u_{n}=U, \quad u_{m_{1}}=0 \quad \text { e } \quad u_{m_{2}}=0
$$

ou seja, a componente normal da velocidade tem um valor não nulo prescrito no injetor, enquanto que as componentes tangenciais da velocidade têm valor nulo.

Para as componentes do tensor não-Newtoniano adotamos a estratégia de apresentada por Mompean [50], que impõe um valor nulo para as componentes do tensor no injetor:

$$
S^{x x}=0, \quad S^{y y}=0, \quad S^{z z}=0, \quad S^{x y}=0, \quad S^{x z}=0 \quad \text { e } \quad S^{y z}=0 .
$$

\section{- Contornos de ejeção}

No ejetor impõem-se a condição homogênea de Neumann para as componentes da velocidade e do tensor não-Newtoniano, ou seja:

$$
\frac{\partial u_{n}}{\partial n}=0, \quad \frac{\partial u_{m_{1}}}{\partial n}=0 \quad \text { e } \quad \frac{\partial u_{m_{2}}}{\partial n}=0
$$




$$
\frac{\partial S^{x x}}{\partial n}=\frac{\partial S^{y y}}{\partial n}=\frac{\partial S^{z z}}{\partial n}=\frac{\partial S^{x y}}{\partial n}=\frac{\partial S^{x z}}{\partial n}=\frac{\partial S^{y z}}{\partial n}=0
$$

O subescrito $n$ denota a direção normal ao contorno e os subescritos $m_{1}$ e $m_{2}$ denotam as direções tangenciais ao contorno.

\subsubsection{Condição de contorno na superfície livre}

Neste trabalho consideramos escoamentos transientes com superfícies livres de um fluido viscoso numa atmosfera passiva, na qual pode-se tomar a pressão como sendo nula. Na ausência de efeitos de tensão superficial, as componentes de tensão normal e tangencial devem ser contínuas sobre tais superfícies (ver Batchelor [5]):

$$
\begin{aligned}
& n_{i} \cdot\left(\sigma_{i j} \cdot n_{j}\right)=0, \\
& m_{1 i} \cdot\left(\sigma_{i j} \cdot n_{j}\right)=0, \\
& m_{2 i} \cdot\left(\sigma_{i j} \cdot n_{j}\right)=0, \quad i, j=1,2,3,
\end{aligned}
$$

onde $n_{i}$ denota o vetor unitário normal à superfície livre e $m 1_{i}, m_{2 i}$ denotam os vetores unitários

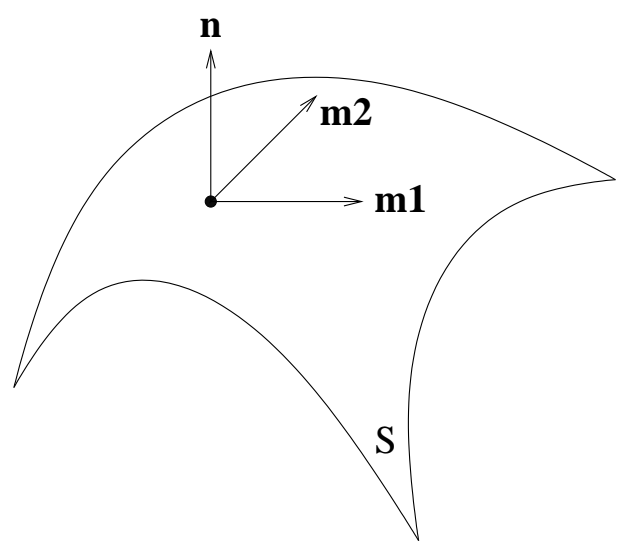

Figura 3.1: Esboço da superfície livre do fluido; $n_{i}$ : vetor unitário normal; $m 1_{i}, m 2_{i}$ : vetores unitários tangenciais à superfície livre do fluido.

tangenciais à superfície livre, (ver a figura 3.1); $\sigma_{i j}$ é o tensor total dado por

$$
\sigma_{i j}=-p \delta_{i j}+2 \eta D_{i j}+S_{i j}, \quad i, j=1,2,3 .
$$

Usando coordenadas cartesianas tridimensionais e tomando $n_{i}=\left(n_{x}, n_{y}, n_{z}\right), m_{1 i}=\left(m_{1 x}, m_{1 y}, m_{1 z}\right)$ e $m_{2 i}=\left(m_{2 x}, m_{2 y}, m_{2 z}\right)$, a condição (3.15) pode ser escrita na forma como

$$
\begin{aligned}
p= & \frac{2}{R e}\left[\frac{\partial u}{\partial x} n_{x}{ }^{2}+\frac{\partial v}{\partial y} n_{y}{ }^{2}+\frac{\partial w}{\partial z} n_{z}{ }^{2}+\left(\frac{\partial u}{\partial y}+\frac{\partial v}{\partial x}\right) n_{x} n_{y}+\left(\frac{\partial u}{\partial z}+\frac{\partial w}{\partial x}\right) n_{x} n_{z}+\left(\frac{\partial v}{\partial z}+\frac{\partial w}{\partial y}\right) n_{y} n_{z}\right] \\
& +S^{x x} n_{x}{ }^{2}+S^{y y} n_{y}{ }^{2}+S^{z z} n_{z}{ }^{2}+2 S^{x y} n_{x} n_{y}+2 S^{x z} n_{x} n_{z}+2 S^{y z} n_{y} n_{z},
\end{aligned}
$$




$$
\begin{aligned}
& \frac{1}{R e}\left[2 \frac{\partial u}{\partial x} n_{x} m_{1 x}+2 \frac{\partial v}{\partial y} n_{y} m_{1 y}+2 \frac{\partial w}{\partial z} n_{z} m_{1 z}+\left(\frac{\partial u}{\partial y}+\frac{\partial v}{\partial x}\right)\left(n_{x} m_{1 y}+n_{y} m_{1 x}\right)\right. \\
& \left.+\left(\frac{\partial u}{\partial z}+\frac{\partial w}{\partial x}\right)\left(n_{x} m_{1 z}+n_{z} m_{1 x}\right)+\left(\frac{\partial v}{\partial z}+\frac{\partial w}{\partial y}\right)\left(n_{y} m_{1 z}+n_{z} m_{1 y}\right)\right]+S^{x x} n_{x} m_{1 x}+S^{y y} n_{y} m_{1 y} \\
& +S^{z z} n_{z} m_{1 z}+S^{x y}\left(n_{x} m_{1 y}+n_{y} m_{1 x}\right)+S^{x z}\left(n_{x} m_{1 z}+n_{z} m_{1 x}\right)+S^{y z}\left(n_{y} m_{1 z}+n_{z} m_{1 y}\right)=0 \\
& \frac{1}{R e}\left[2 \frac{\partial u}{\partial x} n_{x} m_{2 x}+2 \frac{\partial v}{\partial y} n_{y} m_{2 y}+2 \frac{\partial w}{\partial z} n_{z} m_{2 z}+\left(\frac{\partial u}{\partial y}+\frac{\partial v}{\partial x}\right)\left(n_{x} m_{2 y}+n_{y} m_{2 x}\right)\right. \\
& \left.+\left(\frac{\partial u}{\partial z}+\frac{\partial w}{\partial x}\right)\left(n_{x} m_{2 z}+n_{z} m_{2 x}\right)+\left(\frac{\partial v}{\partial z}+\frac{\partial w}{\partial y}\right)\left(n_{y} m_{2 z}+n_{z} m_{2 y}\right)\right]+S^{x x} n_{x} m_{2 x}+S^{y y} n_{y} m_{2 y} \\
& +S^{z z} n_{z} m_{2 z}+S^{x y}\left(n_{x} m_{2 y}+n_{y} m_{2 x}\right)+S^{x z}\left(n_{x} m_{2 z}+n_{z} m_{2 x}\right)+S^{y z}\left(n_{y} m_{2 z}+n_{z} m_{2 y}\right)=0 .
\end{aligned}
$$

\subsection{Cálculo do tensor não-Newtoniano em contornos rígi- dos}

Em contornos rígidos estacionários é suficiente aplicar a condição de não-escorregamento para as componentes da velocidade:

$$
u=0, \quad v=0 \quad \text { e } \quad w=0 .
$$

Ao calcular as componentes do tensor não-Newtoniano $S_{i j}$ por meio das equações (3.9-(3.14), os termos convectivos serão aproximados utilizando um método 'upwind' de alta ordem. Nesse trabalho vamos utilizar o método CUBISTA [1], o qual requer os valores ajusante e a montante da variável que está sendo aproximada. Isso faz com que os valores das variáveis nos contornos rígidos sejam necessários (ver capítulo 3, seção 2.5). As componentes do tensor não-Newtoniano $S_{i j}$ são calculadas no contorno rígido a partir das equações (3.9)-(3.14) seguindo as idéias apresentadas para o caso bidimensional, como segue. Fazendo a mudança de variável

$$
S_{i j}=e^{-\frac{1}{W e} t} \tilde{S}_{i j}
$$


as equações (3.9)-(3.14) reduzem-se a

$$
\begin{aligned}
\frac{\partial \tilde{S}^{x x}}{\partial t} & =-R e \varepsilon e^{-\frac{1}{W e} t}\left(\tilde{S}^{x x}+\tilde{S}^{y y}+\tilde{S}^{z z}\right) \tilde{S}^{x x}-\frac{\partial\left(u \tilde{S}^{x x}\right)}{\partial x}-\frac{\partial\left(v \tilde{S}^{x x}\right)}{\partial y}-\frac{\partial\left(w \tilde{S}^{x x}\right)}{\partial z} \\
& +2(1-\xi) D^{x x} \tilde{S}^{x x}+\left[(2-\xi) \frac{\partial u}{\partial y}-\xi \frac{\partial v}{\partial x}\right] \tilde{S}^{x y}+\left[(2-\xi) \frac{\partial u}{\partial z}-\xi \frac{\partial w}{\partial x}\right] \tilde{S}^{x z} \\
& -2 \varepsilon\left(\tilde{S}^{x x}+\tilde{S}^{y y}+\tilde{S}^{z z}\right) D^{x x}-\frac{2}{R e} e^{-\frac{1}{W e} t}\left\{\frac{\partial\left(D^{x x}\right)}{\partial t}+\frac{\partial\left(u D^{x x}\right)}{\partial x}+\frac{\partial\left(v D^{x x}\right)}{\partial y}+\frac{\partial\left(w D^{x x}\right)}{\partial z}\right. \\
& \left.-2(1-\xi)\left(D^{x x}\right)^{2}-\left[(2-\xi) \frac{\partial u}{\partial y}-\xi \frac{\partial v}{\partial x}\right] D^{x y}-\left[(2-\xi) \frac{\partial u}{\partial z}-\xi \frac{\partial w}{\partial x}\right] D^{x z},\right\}
\end{aligned}
$$

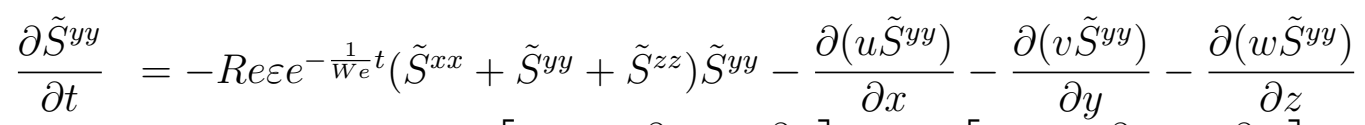

$$
\begin{aligned}
& +2(1-\xi) D^{y y} \tilde{S}^{y y}+\left[(2-\xi) \frac{\partial v}{\partial x}-\xi \frac{\partial u}{\partial y}\right] \tilde{S}^{x y}+\left[(2-\xi) \frac{\partial v}{\partial z}-\xi \frac{\partial w}{\partial y}\right] \tilde{S}^{y z} \\
& -2 \varepsilon\left(\tilde{S}^{x x}+\tilde{S}^{y y}+\tilde{S}^{z z}\right) D^{y y}-\frac{2}{R e} e^{-\frac{1}{W e} t}\left\{\frac{\partial\left(D^{y y}\right)}{\partial t}+\frac{\partial\left(u D^{y y}\right)}{\partial x}+\frac{\partial\left(v D^{y y}\right)}{\partial y}+\frac{\partial\left(w D^{y y}\right)}{\partial z}\right. \\
& \left.-2(1-\xi)\left(D^{y y}\right)^{2}-\left[(2-\xi) \frac{\partial v}{\partial x}-\xi \frac{\partial u}{\partial y}\right] D^{x y}-\left[(2-\xi) \frac{\partial v}{\partial z}-\xi \frac{\partial w}{\partial y}\right] D^{y z}\right\},
\end{aligned}
$$

$$
\begin{aligned}
& \frac{\partial \tilde{S}^{z z}}{\partial t}=-\operatorname{Re}^{-\frac{1}{W e} t}\left(\tilde{S}^{x x}+\tilde{S}^{y y}+\tilde{S}^{z z}\right) \tilde{S}^{z z}-\frac{\partial\left(u \tilde{S}^{z z}\right)}{\partial x}-\frac{\partial\left(v \tilde{S}^{z z}\right)}{\partial y}-\frac{\partial\left(w \tilde{S}^{z z}\right)}{\partial z} \\
& +2(1-\xi) D^{z z} \tilde{S}^{z z}+\left[(2-\xi) \frac{\partial w}{\partial x}-\xi \frac{\partial u}{\partial z}\right] \tilde{S}^{x z}+\left[(2-\xi) \frac{\partial w}{\partial y}-\xi \frac{\partial v}{\partial z}\right] \tilde{S}^{y z} \\
& -2 \varepsilon\left(\tilde{S}^{x x}+\tilde{S}^{y y}+\tilde{S}^{z z}\right) D^{z z}-\frac{2}{R e} e^{-\frac{1}{W e} t}\left\{\frac{\partial\left(D^{z z}\right)}{\partial t}+\frac{\partial\left(u D^{z z}\right)}{\partial x}+\frac{\partial\left(v D^{z z}\right)}{\partial y}+\frac{\partial\left(w D^{z z}\right)}{\partial z}\right. \\
& \left.-2(1-\xi)\left(D^{z z}\right)^{2}-\left[(2-\xi) \frac{\partial w}{\partial x}-\xi \frac{\partial u}{\partial z}\right] D^{x z}-\left[(2-\xi) \frac{\partial w}{\partial y}-\xi \frac{\partial v}{\partial z}\right] D^{y z}\right\},
\end{aligned}
$$

$$
\begin{aligned}
\frac{\partial \tilde{S}^{x y}}{\partial t} & =-R e \varepsilon e^{-\frac{1}{W e} t}\left(\tilde{S}^{x x}+\tilde{S}^{y y}+\tilde{S}^{z z}\right) \tilde{S}^{x y}-\frac{\partial\left(u \tilde{S}^{x y}\right)}{\partial x}-\frac{\partial\left(v \tilde{S}^{x y}\right)}{\partial y}-\frac{\partial\left(w \tilde{S}^{x y}\right)}{\partial z} \\
+ & (1-\xi)\left(D^{x x}+D^{y y}\right) \tilde{S}^{x y}+\left[\left(1-\frac{\xi}{2}\right) \frac{\partial v}{\partial x}-\frac{\xi}{2} \frac{\partial u}{\partial y}\right] \tilde{S}^{x x} \\
+ & {\left[\left(1-\frac{\xi}{2}\right) \frac{\partial u}{\partial y}-\frac{\xi}{2} \frac{\partial v}{\partial x}\right] \tilde{S}^{y y}+\left[\left(1-\frac{\xi}{2}\right) \frac{\partial v}{\partial z}-\frac{\xi}{2} \frac{\partial w}{\partial y}\right] \tilde{S}^{x z} } \\
+ & {\left[\left(1-\frac{\xi}{2}\right) \frac{\partial u}{\partial z}-\frac{\xi}{2} \frac{\partial w}{\partial x}\right] \tilde{S}^{y z}-2 \varepsilon\left(\tilde{S}^{x x}+\tilde{S}^{y y}+\tilde{S}^{z z}\right) D^{x y} } \\
& -\frac{2}{R e} e^{-\frac{1}{W e} t}\left\{\frac{\partial\left(D^{x y}\right)}{\partial t}+\frac{\partial\left(u D^{x y}\right)}{\partial x}+\frac{\partial\left(v D^{x y}\right)}{\partial y}+\frac{\partial\left(w D^{x y}\right)}{\partial z}-(1-\xi)\left(D^{x x}+D^{y y}\right) D^{x y}\right. \\
& -\left[\left(1-\frac{\xi}{2}\right) \frac{\partial v}{\partial x}-\frac{\xi}{2} \frac{\partial u}{\partial y}\right] D^{x x}-\left[\left(1-\frac{\xi}{2}\right) \frac{\partial u}{\partial y}-\frac{\xi}{2} \frac{\partial v}{\partial x}\right] D^{y y} \\
& \left.-\left[\left(1-\frac{\xi}{2}\right) \frac{\partial v}{\partial z}-\frac{\xi}{2} \frac{\partial w}{\partial y}\right] D^{x z}-\left[\left(1-\frac{\xi}{2}\right) \frac{\partial u}{\partial z}-\frac{\xi}{2} \frac{\partial w}{\partial x}\right] D^{y z}\right\}
\end{aligned}
$$




$$
\begin{aligned}
& \frac{\partial \tilde{S}^{x z}}{\partial t}=-\operatorname{Re} \varepsilon e^{-\frac{1}{W e} t}\left(\tilde{S}^{x x}+\tilde{S}^{y y}+\tilde{S}^{z z}\right) \tilde{S}^{x z}-\frac{\partial\left(u \tilde{S}^{x z}\right)}{\partial x}-\frac{\partial\left(v \tilde{S}^{x z}\right)}{\partial y}-\frac{\partial\left(w \tilde{S}^{x z}\right)}{\partial z} \\
& +(1-\xi)\left(D^{x x}+D^{z z}\right) \tilde{S}^{x z}+\left[\left(1-\frac{\xi}{2}\right) \frac{\partial u}{\partial y}-\frac{\xi}{2} \frac{\partial v}{\partial x}\right] \tilde{S}^{y z} \\
& +\left[\left(1-\frac{\xi}{2}\right) \frac{\partial w}{\partial x}-\frac{\xi}{2} \frac{\partial u}{\partial z}\right] \tilde{S}^{x x}+\left[\left(1-\frac{\xi}{2}\right) \frac{\partial u}{\partial z}-\frac{\xi}{2} \frac{\partial w}{\partial x}\right] \tilde{S}^{z z} \\
& +\left[\left(1-\frac{\xi}{2}\right) \frac{\partial w}{\partial y}-\frac{\xi}{2} \frac{\partial v}{\partial z}\right] \tilde{S}^{x y}-2 \varepsilon\left(\tilde{S}^{x x}+\tilde{S}^{y y}+\tilde{S}^{z z}\right) D^{x z} \\
& -\frac{2}{R e} e^{-\frac{1}{W e} t}\left\{\frac{\partial\left(D^{x z}\right)}{\partial t}+\frac{\partial\left(u D^{x z}\right)}{\partial x}+\frac{\partial\left(v D^{x z}\right)}{\partial y}+\frac{\partial\left(w D^{x z}\right)}{\partial z}-(1-\xi)\left(D^{x x}+D^{z z}\right) D^{x z}\right. \\
& -\left[\left(1-\frac{\xi}{2}\right) \frac{\partial u}{\partial y}-\frac{\xi}{2} \frac{\partial v}{\partial x}\right] D^{y z}-\left[\left(1-\frac{\xi}{2}\right) \frac{\partial w}{\partial x}-\frac{\xi}{2} \frac{\partial u}{\partial z}\right] D^{x x} \\
& \left.-\left[\left(1-\frac{\xi}{2}\right) \frac{\partial u}{\partial z}-\frac{\xi}{2} \frac{\partial w}{\partial x}\right] D^{z z}-\left[\left(1-\frac{\xi}{2}\right) \frac{\partial w}{\partial y}-\frac{\xi}{2} \frac{\partial v}{\partial z}\right] D^{x y}\right\}, \\
& \frac{\partial \tilde{S}^{y z}}{\partial t}=-\operatorname{Re} \varepsilon e^{-\frac{1}{W e} t}\left(\tilde{S}^{x x}+\tilde{S}^{y y}+\tilde{S}^{z z}\right) \tilde{S}^{y z}-\frac{\partial\left(u \tilde{S}^{y z}\right)}{\partial x}-\frac{\partial\left(v \tilde{S}^{y z}\right)}{\partial y}-\frac{\partial\left(w \tilde{S}^{y z}\right)}{\partial z} \\
& +(1-\xi)\left(D^{y y}+D^{z z}\right) \tilde{S}^{y z}+\left[\left(1-\frac{\xi}{2}\right) \frac{\partial v}{\partial z}-\frac{\xi}{2} \frac{\partial w}{\partial y}\right] \tilde{S}^{z z} \\
& +\left[\left(1-\frac{\xi}{2}\right) \frac{\partial w}{\partial y}-\frac{\xi}{2} \frac{\partial v}{\partial z}\right] \tilde{S}^{y y}+\left[\left(1-\frac{\xi}{2}\right) \frac{\partial w}{\partial x}-\frac{\xi}{2} \frac{\partial u}{\partial z}\right] \tilde{S}^{x y} \\
& +\left[\left(1-\frac{\xi}{2}\right) \frac{\partial v}{\partial x}-\frac{\xi}{2} \frac{\partial u}{\partial y}\right] \tilde{S}^{x z}-2 \varepsilon\left(\tilde{S}^{x x}+\tilde{S}^{y y}+\tilde{S}^{z z}\right) D^{y z} \\
& -\frac{2}{R e} e^{-\frac{1}{W e} t}\left\{\frac{\partial\left(D^{x z}\right)}{\partial t}+\frac{\partial\left(u \tilde{S}^{y z}\right)}{\partial x}+\frac{\partial\left(v \tilde{S}^{y z}\right)}{\partial y}+\frac{\partial\left(w \tilde{S}^{y z}\right)}{\partial z}-(1-\xi)\left(D^{y y}+D^{z z}\right) \tilde{S}^{y z}\right. \\
& -\left[\left(1-\frac{\xi}{2}\right) \frac{\partial v}{\partial z}-\frac{\xi}{2} \frac{\partial w}{\partial y}\right] \tilde{S}^{z z}-\left[\left(1-\frac{\xi}{2}\right) \frac{\partial w}{\partial y}-\frac{\xi}{2} \frac{\partial v}{\partial z}\right] \tilde{S}^{y y} \\
& \left.-\left[\left(1-\frac{\xi}{2}\right) \frac{\partial w}{\partial x}-\frac{\xi}{2} \frac{\partial u}{\partial z}\right] \tilde{S}^{x y}-\left[\left(1-\frac{\xi}{2}\right) \frac{\partial v}{\partial x}-\frac{\xi}{2} \frac{\partial u}{\partial y}\right] \tilde{S}^{x z}\right\} \text {. }
\end{aligned}
$$




\subsubsection{Contornos rígidos paralelos ao plano- $x y$}

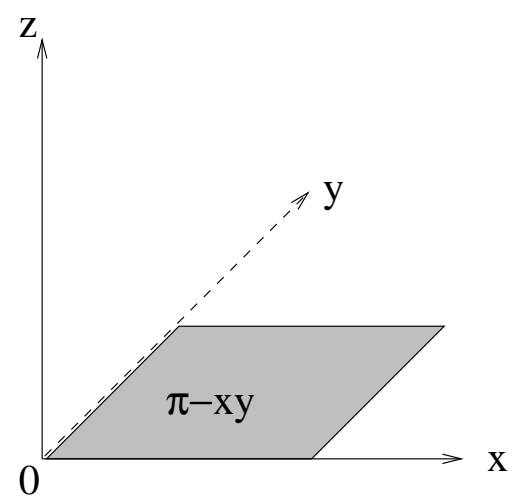

Figura 3.2: Plano-xy $(\pi-x y)$.

Da condição não-escorregamento obtemos:

$$
\frac{\partial}{\partial x}=\frac{\partial}{\partial y}=0 \Rightarrow \frac{\partial w}{\partial z}=0
$$

Assim, somente os termos $\frac{\partial u}{\partial z}$ e $\frac{\partial v}{\partial z}$ são não-nulos. Nesse caso, as equações (3.19)-(3.24) reduzem-se a

$$
\begin{aligned}
& \frac{\partial \tilde{S}^{x x}}{\partial t}=-\operatorname{Re} \varepsilon e^{-\frac{1}{W e} t}\left(\tilde{S}^{x x}+\tilde{S}^{y y}+\tilde{S}^{z z}\right) \tilde{S}^{x x}+(2-\xi) \frac{\partial u}{\partial z} \tilde{S}^{x z}+\frac{e^{\frac{1}{W e} t}}{R e}(2-\xi)\left(\frac{\partial u}{\partial z}\right)^{2}, \\
& \frac{\partial \tilde{S}^{y y}}{\partial t}=\tilde{S}^{y y}+(2-\xi) \frac{\partial v}{\partial z} \tilde{S}^{y z}+\frac{e^{\frac{1}{W e} t}}{R e}(2-\xi)\left(\frac{\partial v}{\partial z}\right)^{2}, \\
& \frac{\partial \tilde{S}^{z z}}{\partial t}=-\operatorname{Re} \varepsilon e^{-\frac{1}{W e} t}\left(\tilde{S}^{x x}+\tilde{S}^{y y}+\tilde{S}^{z z}\right) \tilde{S}^{z z}-\xi \frac{\partial u}{\partial z} \tilde{S}^{x z}-\xi \frac{\partial v}{\partial z} \tilde{S}^{y z}-\frac{e^{\frac{1}{W e} t}}{\operatorname{Re}} \xi\left[\left(\frac{\partial u}{\partial z}\right)^{2}+\left(\frac{\partial v}{\partial z}\right)^{2}\right], \\
& \frac{\partial \tilde{S}^{x y}}{\partial t}=-\operatorname{Re} \varepsilon e^{-\frac{1}{W e} t}\left(\tilde{S}^{x x}+\tilde{S}^{y y}+\tilde{S}^{z z}\right) \tilde{S}^{x y}+\left(1-\frac{\xi}{2}\right) \frac{\partial u}{\partial z} \tilde{S}^{y z}+\left(1-\frac{\xi}{2}\right) \frac{\partial v}{\partial z} \tilde{S}^{x z} \\
& +\frac{e^{\frac{1}{W e} t}}{R e}(2-\xi) \frac{\partial u}{\partial z} \frac{\partial v}{\partial z} \\
& \frac{\partial \tilde{S}^{x z}}{\partial t}=-\operatorname{Re} \varepsilon e^{-\frac{1}{W e} t}\left(\tilde{S}^{x x}+\tilde{S}^{y y}+\tilde{S}^{z z}\right) \tilde{S}^{x z}-\frac{\xi}{2} \frac{\partial u}{\partial z} \tilde{S}^{x x}+\left(1-\frac{\xi}{2}\right) \frac{\partial u}{\partial z} \tilde{S}^{z z}-\frac{\xi}{2} \frac{\partial v}{\partial z} \tilde{S}^{x y} \\
& +\frac{1}{R e} e^{\frac{1}{W e} t} \frac{\partial u}{\partial z}-\varepsilon \frac{\partial u}{\partial z}\left(\tilde{S}^{x x}+\tilde{S}^{y y}+\tilde{S}^{z z}\right)-\frac{e^{\frac{1}{W e} t}}{R e} \frac{\partial}{\partial t}\left(\frac{\partial u}{\partial z}\right), \\
& \frac{\partial \tilde{S}^{y z}}{\partial t}=-\operatorname{Re} \varepsilon e^{-\frac{1}{W e} t}\left(\tilde{S}^{x x}+\tilde{S}^{y y}+\tilde{S}^{z z}\right) \tilde{S}^{y z}-\frac{\xi}{2} \frac{\partial v}{\partial z} \tilde{S}^{y y}+\left(1-\frac{\xi}{2}\right) \frac{\partial v}{\partial z} \tilde{S}^{z z}-\frac{\xi}{2} \frac{\partial u}{\partial z} \tilde{S}^{x y} \\
& +\frac{1}{R e} e^{\frac{1}{W e} t} \frac{\partial v}{\partial z}-\varepsilon \frac{\partial v}{\partial z}\left(\tilde{S}^{x x}+\tilde{S}^{y y}+\tilde{S}^{z z}\right)-\frac{e^{\frac{1}{W e} t}}{R e} \frac{\partial}{\partial t}\left(\frac{\partial v}{\partial z}\right)
\end{aligned}
$$

Os termos $e^{-\frac{1}{W e} t}\left(\tilde{S}^{x x}+\tilde{S}^{y y}+\tilde{S}^{z z}\right) \tilde{S}^{l m}$ nas equações (3.25)-(3.30) fazem com que essas equações formem um sistema não-linear $6 \times 6$. Para evitar esse sistema não-linear, observamos que $o$ 
termo $e^{-\frac{1}{W e} t}<<1$ e portanto esses termos são desprezados fazendo com que essas equações resultem no seguinte sistema linear $6 \times 6$ :

$$
\begin{aligned}
\frac{\partial \tilde{S}^{x x}}{\partial t} & =(2-\xi) \frac{\partial u}{\partial z} \tilde{S}^{x z}+\frac{e^{\frac{1}{W e} t}}{R e}(2-\xi)\left(\frac{\partial u}{\partial z}\right)^{2} \\
\frac{\partial \tilde{S}^{y y}}{\partial t} & =(2-\xi) \frac{\partial v}{\partial z} \tilde{S}^{y z}+\frac{e^{\frac{1}{W e} t}}{R e}(2-\xi)\left(\frac{\partial v}{\partial z}\right)^{2} \\
\frac{\partial \tilde{S}^{z z}}{\partial t} & \left.=-\xi \frac{\partial u}{\partial z} \tilde{S}^{x z}-\xi \frac{\partial v}{\partial z} \tilde{S}^{y z}-\frac{e^{\frac{1}{W e} t}}{R e} \xi\left(\frac{\partial u}{\partial z}\right)^{2}+\left(\frac{\partial v}{\partial z}\right)^{2}\right] \\
\frac{\partial \tilde{S}^{x y}}{\partial t} & =\left(1-\frac{\xi}{2}\right) \frac{\partial u}{\partial z} \tilde{S}^{y z}+\left(1-\frac{\xi}{2}\right) \frac{\partial v}{\partial z} \tilde{S}^{x z}+\frac{e^{\frac{1}{W e} t}}{R e}(2-\xi) \frac{\partial u}{\partial z} \frac{\partial v}{\partial z} \\
\frac{\partial \tilde{S}^{x z}}{\partial t} & =-\frac{\xi}{2} \frac{\partial u}{\partial z} \tilde{S}^{x x}+\left(1-\frac{\xi}{2}\right) \frac{\partial u}{\partial z} \tilde{S}^{z z}-\frac{\xi}{2} \frac{\partial v}{\partial z} \tilde{S}^{x y}+\frac{1}{R e} e^{\frac{1}{W e} t} \frac{\partial u}{\partial z}-\varepsilon \frac{\partial u}{\partial z}\left(\tilde{S}^{x x}+\tilde{S}^{y y}+\tilde{S}^{z z}\right) \\
& -\frac{e^{\frac{1}{W e} t}}{R e} \frac{\partial}{\partial t}\left(\frac{\partial u}{\partial z}\right), \\
\frac{\partial \tilde{S}^{y z}}{\partial t} & =-\frac{\xi}{2} \frac{\partial v}{\partial z} \tilde{S}^{y y}+\left(1-\frac{\xi}{2}\right) \frac{\partial v}{\partial z} \tilde{S}^{z z}-\frac{\xi}{2} \frac{\partial u}{\partial z} \tilde{S}^{x y}+\frac{1}{R e} e^{\frac{1}{W e} t} \frac{\partial v}{\partial z}-\varepsilon \frac{\partial v}{\partial z}\left(\tilde{S}^{x x}+\tilde{S}^{y y}+\tilde{S}^{z z}\right) \\
& -\frac{e^{\frac{1}{W e} t}}{R e} \frac{\partial}{\partial t}\left(\frac{\partial v}{\partial z}\right)
\end{aligned}
$$

Para obtermos expressões para as componentes $\tilde{S}^{x x}, \tilde{S}^{y y}, \tilde{S}^{z z}, \tilde{S}^{x y}, \tilde{S}^{x z}$ e $\tilde{S}^{y z}$ vamos integrar as equações (3.31)-(3.36) no intervalo $[t, t+\delta t]$ como segue.

As integrais da forma

$$
\int_{t}^{t+\delta t}\left(\frac{\partial u}{\partial z} \tilde{S}^{l m}\right)(x, y, z, s) d s \text { e } \int_{t}^{t+\delta t}\left(\frac{\partial v}{\partial z} \tilde{S}^{l m}\right)(x, y, z, s) d s
$$

em que $l$ e $m$ representa $x$ ou $y$, são aproximadas pela "fórmula dos trapézios", ou seja:

$$
\begin{aligned}
\int_{t}^{t+\delta t}\left(\frac{\partial u}{\partial z} \tilde{S}^{l m}\right)(x, y, z, s) d s & =\frac{\delta t}{2}\left[\left(\frac{\partial u}{\partial z} \tilde{S}^{l m}\right)(x, y, z, t)+\left(\frac{\partial u}{\partial z} \tilde{S}^{l m}\right)(x, y, z, t+\delta t)\right] \\
\int_{t}^{t+\delta t}\left(\frac{\partial v}{\partial z} \tilde{S}^{l m}\right)(x, y, z, s) d s & =\frac{\delta t}{2}\left[\left(\frac{\partial v}{\partial z} \tilde{S}^{l m}\right)(x, y, z, t)+\left(\frac{\partial v}{\partial z} \tilde{S}^{l m}\right)(x, y, z, t+\delta t)\right],
\end{aligned}
$$

enquanto as integrais da forma $\int_{t}^{t+\delta t} e^{\frac{1}{W e} s} \frac{\partial u(x, y, z, s)}{\partial z} d s$ e $\int_{t}^{t+\delta t} e^{\frac{1}{W e} s} \frac{\partial v(x, y, z, s)}{\partial z} d s$ são resolvidas utilizando o "teorema do valor médio pra integrais", ou seja,

$$
\begin{aligned}
\int_{t}^{t+\delta t} e^{\frac{1}{W e} s} \frac{\partial u(x, y, z, s)}{\partial z} d s & =e^{\frac{1}{W e} t}\left(e^{\frac{1}{W e} \delta t}-1\right) W e \frac{\partial u\left(x, y, z, t^{*}\right)}{\partial z} \\
\int_{t}^{t+\delta t} e^{\frac{1}{W e} s} \frac{\partial v(x, y, z, s)}{\partial z} d s & =e^{\frac{1}{W e} t}\left(e^{\frac{1}{W e} \delta t}-1\right) W e \frac{\partial v\left(x, y, z, t^{*}\right)}{\partial z}
\end{aligned}
$$


e as integrais da forma $\int_{t}^{t+\delta t} e^{\frac{1}{W e} s} \frac{\partial}{\partial s}\left(\frac{\partial u(x, y, z, s)}{\partial z}\right) d s$ e $\int_{t}^{t+\delta t} e^{\frac{1}{W e} s} \frac{\partial}{\partial s}\left(\frac{\partial v(x, y, z, s)}{\partial z}\right) d s$ são resolvidas por "integração por partes", isto é:

$$
\begin{aligned}
\int_{t}^{t+\delta t} e^{\frac{1}{W e} s} \frac{\partial}{\partial s}\left(\frac{\partial u(x, y, z, s)}{\partial z}\right) d s & =e^{\frac{1}{W e} t}\left[e^{\frac{1}{W e} \delta t} \frac{\partial u(x, y, z, t+\delta t)}{\partial z}-\frac{\partial u(x, y, z, t)}{\partial z}\right. \\
& \left.-\left(e^{\frac{1}{W e} \delta t}-1\right) \frac{\partial u\left(x, y, z, t^{*}\right)}{\partial z}\right], \\
\int_{t}^{t+\delta t} e^{\frac{1}{W e}} s \frac{\partial}{\partial s}\left(\frac{\partial v(x, y, z, s)}{\partial z}\right) d s & =e^{\frac{1}{W e} t}\left[e^{\frac{1}{W e} \delta t} \frac{\partial v(x, y, z, t+\delta t)}{\partial z}-\frac{\partial v(x, y, z, t)}{\partial z}\right. \\
& \left.-\left(e^{\frac{1}{W e} \delta t}-1\right) \frac{\partial v\left(x, y, z, t^{*}\right)}{\partial z}\right]
\end{aligned}
$$

onde

$$
\begin{aligned}
& \frac{\partial u\left(x, y, z, t^{*}\right)}{\partial z}=\frac{1}{2}\left[\frac{\partial u(x, y, z, t)}{\partial z}+\frac{\partial u(x, y, z, t+\delta t)}{\partial z}\right] \\
& \frac{\partial v\left(x, y, z, t^{*}\right)}{\partial z}=\frac{1}{2}\left[\frac{\partial u(x, y, z, t)}{\partial z}+\frac{\partial v(x, y, z, t+\delta t)}{\partial z}\right]
\end{aligned}
$$

Dessa maneira, integrando as equações (3.31)-(3.36) no intervalo $[t, t+\delta t]$ obtemos:

$$
\begin{aligned}
& \tilde{S}^{x x}{ }^{(n+1)}=\tilde{S}^{x x}+(2-\xi) \frac{\delta t}{2}\left(\frac{\partial u}{\partial z} \tilde{S}^{x z}+\frac{\partial u^{(n+1)}}{\partial z} \tilde{S}^{(n z+1)}\right)+(2-\xi) \frac{W e}{R e} e^{\frac{1}{W e} t}\left(e^{\frac{1}{W e} \delta t}-1\right)\left(\frac{\partial u\left(t^{*}\right)}{\partial z}\right)^{2} \\
& \tilde{S} y y^{(n+1)}=\tilde{S}^{y y}+(2-\xi) \frac{\delta t}{2}\left(\frac{\partial v}{\partial z} \tilde{S}^{y z}+\frac{\partial v^{(n+1)}}{\partial z} \tilde{S}^{(n+1)}\right)+(2-\xi) \frac{W e}{R e} e^{\frac{1}{W e} t}\left(e^{\frac{1}{W e} \delta t}-1\right)\left(\frac{\partial v\left(t^{*}\right)}{\partial z}\right)^{2} \\
& \tilde{S}^{(n+1)}=\tilde{S}^{z z}-\xi \frac{\delta t}{2}\left(\frac{\partial u}{\partial z} \tilde{S}^{x z}+\frac{\partial u^{(n+1)}}{\partial z} \tilde{S}^{(n+1)}\right)-\xi \frac{\delta t}{2}\left(\frac{\partial v}{\partial z} \tilde{S}^{y z}+\frac{\partial v^{(n+1)}}{\partial z} \tilde{S}^{(n+1)}\right) \\
& -\xi \frac{W e}{R e} e^{\frac{1}{W e} t}\left(e^{\frac{1}{W e} \delta t}-1\right)\left[\left(\frac{\partial u\left(t^{*}\right)}{\partial z}\right)^{2}+\left(\frac{\partial v\left(t^{*}\right)}{\partial z}\right)^{2}\right] \\
& \tilde{S}^{x y}{ }^{(n+1)}=\tilde{S}^{x y}+\left(1-\frac{\xi}{2}\right) \frac{\delta t}{2}\left(\frac{\partial u}{\partial z} \tilde{S}^{y z}+\frac{\partial u^{(n+1)}}{\partial z} \tilde{S}^{(n+1)}+\frac{\partial v}{\partial z} \tilde{S}^{x z}+\frac{\partial v^{(n+1)}}{\partial z} \tilde{S}^{(n+1)}\right) \\
& +(2-\xi) \frac{W e}{R e} e^{\frac{1}{W e} t}\left(e^{\frac{1}{W e} \delta t}-1\right) \frac{\partial u\left(t^{*}\right)}{\partial z} \frac{\partial v\left(t^{*}\right)}{\partial z},
\end{aligned}
$$




$$
\begin{aligned}
& \tilde{S}^{(n+1)}=\tilde{S}^{x z}-\frac{\xi}{2} \frac{\delta t}{2}\left(\frac{\partial u}{\partial z} \tilde{S}^{x x}+\frac{\partial u^{(n+1)}}{\partial z} \tilde{S}^{(n+1)}+\frac{\partial v}{\partial z} \tilde{S}^{x y}+\frac{\partial v^{(n+1)}}{\partial z} \tilde{S}^{(n+1)}\right) \\
& +\left(1-\frac{\xi}{2}\right) \frac{\delta t}{2}\left(\frac{\partial u}{\partial z} \tilde{S}^{z z}+\frac{\partial u^{(n+1)}}{\partial z} \tilde{S_{z z}}{ }^{(n+1)}\right) \\
& -\varepsilon \frac{\delta t}{2}\left[\frac{\partial u}{\partial z}\left(\tilde{S}^{x x}+\tilde{S}^{y y}+\tilde{S}^{z z}\right)+\frac{\partial u^{(n+1)}}{\partial z}\left({\tilde{S^{x}}}^{(n+1)}+{\tilde{S^{y} y}}^{(n+1)}+\tilde{S}^{(n+1)}\right)\right] \\
& -\frac{e^{\frac{1}{W e}} t}{R e}\left[e^{\frac{1}{W e} \delta t} \frac{\partial u^{(n+1)}}{\partial z}-\frac{\partial u}{\partial z}-\left(e^{\frac{1}{W e} \delta t}-1\right) \frac{\partial u\left(t^{*}\right)}{\partial z}\right] \text {, } \\
& \tilde{S}^{(n+1)}=\tilde{S}^{y z}-\frac{\xi}{2} \frac{\delta t}{2}\left(\frac{\partial v}{\partial z} \tilde{S}^{y y}+\frac{\partial v^{(n+1)}}{\partial z} \tilde{S y y}^{(n+1)}+\frac{\partial u}{\partial z} \tilde{S}^{x y}+\frac{\partial u^{(n+1)}}{\partial z} \tilde{S}^{(n+1)}\right) \\
& +\left(1-\frac{\xi}{2}\right) \frac{\delta t}{2}\left(\frac{\partial v}{\partial z} \tilde{S}^{z z}+\frac{\partial u^{(n+1)}}{\partial z} \tilde{S^{z z}}{ }^{(n+1)}\right) \\
& -\varepsilon \frac{\delta t}{2}\left[\frac{\partial v}{\partial z}\left(\tilde{S}^{x x}+\tilde{S}^{y y}+\tilde{S}^{z z}\right)+\frac{\partial v^{(n+1)}}{\partial z}\left(\tilde{S}^{(x x}{ }^{(n+1)}+\tilde{S} y y^{(n+1)}+\tilde{S}^{(n z}{ }^{(n+1)}\right)\right] \\
& -\frac{e^{\frac{1}{W e} t}}{R e}\left[e^{\frac{1}{W e} \delta t} \frac{\partial v^{(n+1)}}{\partial z}-\frac{\partial v}{\partial z}-\left(e^{\frac{1}{W e} \delta t}-1\right) \frac{\partial v\left(t^{*}\right)}{\partial z}\right] .
\end{aligned}
$$

As equações (3.37)-(3.42) produzem um sistema $6 \times 6$ para as componentes $\tilde{S}^{\tilde{l m}^{(n+1)}}$. Multiplicando as equações $(3.37)-(3.42)$ por $e^{-\frac{1}{W e}(t+\delta t)}$ obtemos as equações para as componentes $S^{l m}{ }^{(n+1)}$ como segue.

$$
\begin{aligned}
& S^{x x(n+1)}=e^{-\frac{1}{W e} \delta t} S^{x x}+(2-\xi) \frac{\delta t}{2}\left(\frac{\partial u}{\partial z} e^{-\frac{1}{W e} \delta t} S^{x z}+\frac{\partial u^{(n+1)}}{\partial z} S^{x z(n+1)}\right) \\
& +(2-\xi) \frac{W e}{R e}\left(1-e^{\frac{1}{W e} \delta t}\right)\left(\frac{\partial u\left(t^{*}\right)}{\partial z}\right)^{2}, \\
& S^{y y(n+1)}=e^{-\frac{1}{W e} \delta t} S^{y y}+(2-\xi) \frac{\delta t}{2}\left(\frac{\partial v}{\partial z} e^{-\frac{1}{W e} \delta t} S^{y z}+\frac{\partial v^{(n+1)}}{\partial z} S^{y z(n+1)}\right) \\
& +(2-\xi) \frac{W e}{R e}\left(1-e^{\frac{1}{W e} \delta t}\right)\left(\frac{\partial v\left(t^{*}\right)}{\partial z}\right)^{2} \\
& S^{z z(n+1)}=e^{-\frac{1}{W e} \delta t} S^{z z}-\xi \frac{\delta t}{2}\left(\frac{\partial u}{\partial z} e^{-\frac{1}{W e} \delta t} S^{x z}+\frac{\partial u^{(n+1)}}{\partial z} S^{x z(n+1)}\right) \\
& -\xi \frac{\delta t}{2}\left(\frac{\partial v}{\partial z} e^{-\frac{1}{W e} \delta t} S^{y z}+\frac{\partial v^{(n+1)}}{\partial z} S^{y z(n+1)}\right) \\
& -\xi \frac{W e}{R e}\left(1-e^{\frac{1}{W e} \delta t}\right)\left[\left(\frac{\partial u\left(t^{*}\right)}{\partial z}\right)^{2}+\left(\frac{\partial v\left(t^{*}\right)}{\partial z}\right)^{2}\right],
\end{aligned}
$$




$$
\begin{aligned}
& S^{x y(n+1)}=e^{-\frac{1}{W e} \delta t} S^{x y}+\left(1-\frac{\xi}{2}\right) \frac{\delta t}{2}\left(\frac{\partial u}{\partial z} e^{-\frac{1}{W e} \delta t} S^{y z}+\frac{\partial u^{(n+1)}}{\partial z} S^{y z(n+1)}\right) \\
& +\left(1-\frac{\xi}{2}\right) \frac{\delta t}{2}\left(\frac{\partial v}{\partial z} e^{-\frac{1}{W e} \delta t} S^{x z}+\frac{\partial v^{(n+1)}}{\partial z} S^{x z(n+1)}\right) \\
& +(2-\xi) \frac{W e}{R e}\left(1-e^{\frac{1}{W e} \delta t}\right) \frac{\partial u\left(t^{*}\right)}{\partial z} \frac{\partial v\left(t^{*}\right)}{\partial z}, \\
& S^{x z(n+1)}=e^{-\frac{1}{W e} \delta t} S^{x z}-\frac{\xi}{2} \frac{\delta t}{2}\left(\frac{\partial u}{\partial z} e^{-\frac{1}{W e} \delta t} S^{x x}+\frac{\partial u^{(n+1)}}{\partial z} S^{x x(n+1)}\right. \\
& \left.+\frac{\partial v}{\partial z} e^{-\frac{1}{W e} \delta t} S^{x y}+\frac{\partial v^{(n+1)}}{\partial z} S^{x y(n+1)}\right)+\left(1-\frac{\xi}{2}\right) \frac{\delta t}{2}\left(\frac{\partial u}{\partial z} e^{-\frac{1}{W e} \delta t} S^{z z}+\frac{\partial u^{(n+1)}}{\partial z} S^{z z(n+1)}\right) \\
& -\varepsilon \frac{\delta t}{2}\left[\frac{\partial u}{\partial z} e^{-\frac{1}{W e} \delta t}\left(S^{x x}+S^{y y}+S^{z z}\right)+\frac{\partial u^{(n+1)}}{\partial z}\left(S^{x x(n+1)}+S^{y y(n+1)}+S^{z z(n+1)}\right)\right] \\
& -\frac{1}{R e}\left[\frac{\partial u^{(n+1)}}{\partial z}-e^{-\frac{1}{W e} \delta t} \frac{\partial u}{\partial z}-\left(1-e^{-\frac{1}{W e} \delta t}\right) \frac{\partial u\left(t^{*}\right)}{\partial z}\right] \text {, } \\
& S^{y z(n+1)}=e^{-\frac{1}{W e} \delta t} S^{y z}-\frac{\xi}{2} \frac{\delta t}{2}\left(\frac{\partial v}{\partial z} e^{-\frac{1}{W e} \delta t} S^{y y}+\frac{\partial v^{(n+1)}}{\partial z} S^{y y(n+1)}\right. \\
& \left.+\frac{\partial u}{\partial z} e^{-\frac{1}{W e} \delta t} S^{x y}+\frac{\partial u^{(n+1)}}{\partial z} S^{x y(n+1)}\right)+\left(1-\frac{\xi}{2}\right) \frac{\delta t}{2}\left(\frac{\partial v}{\partial z} e^{-\frac{1}{W e} \delta t} S^{z z}+\frac{\partial u^{(n+1)}}{\partial z} S^{z z(n+1)}\right) \\
& -\varepsilon \frac{\delta t}{2}\left[\frac{\partial v}{\partial z} e^{\frac{1}{W e} \delta t}\left(S^{x x}+S^{y y}+S^{z z}\right)+\frac{\partial v^{(n+1)}}{\partial z}\left(S^{x x(n+1)}+S^{y y(n+1)}+S^{z z(n+1)}\right)\right] \\
& -\frac{1}{R e}\left[\frac{\partial v^{(n+1)}}{\partial z}-e^{-\frac{1}{W e} \delta t} \frac{\partial v}{\partial z}-\left(1-e^{-\frac{1}{W e} \delta t}\right) \frac{\partial v\left(t^{*}\right)}{\partial z}\right] \text {. }
\end{aligned}
$$

As equações (3.43)-(3.48) formam um sistema linear para as componente do tensor não-Newtoniano no tempo $(n+1)$. Esse sistema pode ser resolvido analiticamente, como segue. Substituindo as equações (3.43)-(3.46) nas equações (3.47) e (3.48) obtemos um sistema linear $2 \times 2$ para as variáveis $S^{x z(n+1)}$ e $S^{y z(n+1)}$ o qual é dado por (3.49):

$$
\left\{\begin{array}{l}
a_{1} X+b_{1} Y=c_{1} \\
a_{2} X+b_{2} Y=c_{2}
\end{array}\right.
$$

onde $X=S^{x z(n+1)}$ e $Y=S^{y z(n+1)}$ são as incógnitas do sistema e $a_{1}, b_{1}, c_{1}, a_{2}, b_{2}$ e $c_{2}$ são as constantes do sistema dadas por 


$$
\begin{aligned}
& a_{1}=1+[\xi(2-\xi)+2 \varepsilon(1-\xi)]\left(\frac{\delta t}{2}\right)^{2}\left(\frac{\partial u^{(n+1)}}{\partial z}\right)^{2}+(2-\xi) \frac{\xi}{4}\left(\frac{\delta t}{2}\right)^{2}\left(\frac{\partial v^{(n+1)}}{\partial z}\right)^{2} \\
& b_{1}=\left[\frac{3 \xi}{4}(2-\xi)+\varepsilon(2-\xi)-\varepsilon \xi\right]\left(\frac{\delta t}{2}\right)^{2} \frac{\partial u^{(n+1)}}{\partial z} \frac{\partial v^{(n+1)}}{\partial z} \\
& c_{1}=e^{-\frac{1}{W e} \delta t}\left\{S^{x z}-\left(\frac{\xi}{2}+\varepsilon\right) \frac{\delta t}{2} \frac{\partial u^{(n+1)}}{\partial z}\left[S^{x x}+(2-\xi) \frac{\delta t}{2} \frac{\partial u}{\partial z} S^{x z}+(2-\xi) \frac{W e}{R e}\left(e^{\frac{1}{W e} \delta t}-1\right)\left(\frac{\partial u\left(t^{*}\right)}{\partial z}\right)^{2}\right]\right. \\
& +\left(1-\frac{\xi}{2}-\varepsilon\right) \frac{\delta t}{2} \frac{\partial u^{(n+1)}}{\partial z}\left[S^{z z}-\xi \frac{\delta t}{2} \frac{\partial u}{\partial z} S^{x z}-\xi \frac{\delta t}{2} \frac{\partial v}{\partial z} S^{y z}-\xi \frac{W e}{R e}\left(e^{\frac{1}{W e} \delta t}-1\right)\left(\left(\frac{\partial u\left(t^{*}\right)}{\partial z}\right)^{2}+\left(\frac{\partial v\left(t^{*}\right)}{\partial z}\right)^{2}\right)\right] \\
& -\frac{\xi}{2} \frac{\delta t}{2} \frac{\partial v^{(n+1)}}{\partial z}\left[S^{x y}+\left(1-\frac{\xi}{2}\right) \frac{\delta t}{2} \frac{\partial u}{\partial z} S^{y z}+\left(1-\frac{\xi}{2}\right) \frac{\delta t}{2} \frac{\partial v}{\partial z} S^{x z}+(2-\xi) \frac{W e}{R e}\left(e^{\frac{1}{W e} \delta t}-1\right) \frac{\partial u\left(t^{*}\right)}{\partial z} \frac{\partial v\left(t^{*}\right)}{\partial z}\right] \\
& -\varepsilon \frac{\delta t}{2} \frac{\partial u^{(n+1)}}{\partial z}\left[S^{y y}+(2-\xi) \frac{\delta t}{2} \frac{\partial v}{\partial z} S^{y z}+(2-\xi) \frac{W e}{R e}\left(e^{\frac{1}{W e} \delta t}-1\right)\left(\frac{\partial v\left(t^{*}\right)}{\partial z}\right)^{2}\right] \\
& -\frac{\xi}{2} \frac{\delta t}{2} \frac{\partial u}{\partial z} S^{x x}-\frac{\xi}{2} \frac{\delta t}{2} \frac{\partial v}{\partial z} S^{x z}+\left(1-\frac{\xi}{2}\right) \frac{\delta t}{2} \frac{\partial u}{\partial z} S^{z z}-\varepsilon \frac{\delta t}{2} \frac{\partial u}{\partial z}\left(S^{x x}+S^{y y}+S^{z z}\right) \\
& \left.-\frac{1}{R e}\left[e^{\frac{1}{W e} \delta t} \frac{\partial u^{(n+1)}}{\partial z}-\frac{\partial u}{\partial z}-\left(e^{\frac{1}{W e} \delta t}-1\right) \frac{\partial u\left(t^{*}\right)}{\partial z}\right]\right\} \\
& a_{2}=\left[\frac{3 \xi}{4}(2-\xi)+\varepsilon(2-\xi)-\varepsilon \xi\right]\left(\frac{\delta t}{2}\right)^{2} \frac{\partial u^{(n+1)}}{\partial z} \frac{\partial v^{(n+1)}}{\partial z} \\
& b_{2}=1+[\xi(2-\xi)+2 \varepsilon(1-\xi)]\left(\frac{\delta t}{2}\right)^{2}\left(\frac{\partial v^{(n+1)}}{\partial z}\right)^{2}+(2-\xi) \frac{\xi}{4}\left(\frac{\delta t}{2}\right)^{2}\left(\frac{\partial u^{(n+1)}}{\partial z}\right)^{2} \\
& c_{2}=e^{-\frac{1}{W e} \delta t}\left\{S^{y z}-\left(\frac{\xi}{2}+\varepsilon\right) \frac{\delta t}{2} \frac{\partial v^{(n+1)}}{\partial z}\left[S^{y y}+(2-\xi) \frac{\delta t}{2} \frac{\partial v}{\partial z} S^{y z}+(2-\xi) \frac{W e}{R e}\left(e^{\frac{1}{W e} \delta t}-1\right)\left(\frac{\partial v\left(t^{*}\right)}{\partial z}\right)^{2}\right]\right. \\
& +\left(1-\frac{\xi}{2}-\varepsilon\right) \frac{\delta t}{2} \frac{\partial v^{(n+1)}}{\partial z}\left[S^{z z}-\xi \frac{\delta t}{2} \frac{\partial u}{\partial z} S^{x z}-\xi \frac{\delta t}{2} \frac{\partial v}{\partial z} S^{y z}-\xi \frac{W e}{R e}\left(e^{\frac{1}{W e} \delta t}-1\right)\left(\left(\frac{\partial u\left(t^{*}\right)}{\partial z}\right)^{2}+\left(\frac{\partial v\left(t^{*}\right)}{\partial z}\right)^{2}\right)\right] \\
& -\frac{\xi}{2} \frac{\delta t}{2} \frac{\partial u^{(n+1)}}{\partial z}\left[S^{x y}+\left(1-\frac{\xi}{2}\right) \frac{\delta t}{2} \frac{\partial u}{\partial z} S^{y z}+\left(1-\frac{\xi}{2}\right) \frac{\delta t}{2} \frac{\partial v}{\partial z} S^{x z}+(2-\xi) \frac{W e}{R e}\left(e^{\frac{1}{W e} \delta t}-1\right) \frac{\partial u\left(t^{*}\right)}{\partial z} \frac{\partial v\left(t^{*}\right)}{\partial z}\right] \\
& -\varepsilon \frac{\delta t}{2} \frac{\partial v^{(n+1)}}{\partial z}\left[S^{x x}+(2-\xi) \frac{\delta t}{2} \frac{\partial u}{\partial z} S^{x z}+(2-\xi) \frac{W e}{R e}\left(e^{\frac{1}{W e} \delta t}-1\right)\left(\frac{\partial u\left(t^{*}\right)}{\partial z}\right)^{2}\right] \\
& -\frac{\xi}{2} \frac{\delta t}{2} \frac{\partial v}{\partial z} S^{y y}-\frac{\xi}{2} \frac{\delta t}{2} \frac{\partial u}{\partial z} S^{x y}+\left(1-\frac{\xi}{2}\right) \frac{\delta t}{2} \frac{\partial v}{\partial z} S^{z z}-\varepsilon \frac{\delta t}{2} \frac{\partial v}{\partial z}\left(S^{x x}+S^{y y}+S^{z z}\right) \\
& \left.-\frac{1}{R e}\left[e^{\frac{1}{W e} \delta t} \frac{\partial v^{(n+1)}}{\partial z}-\frac{\partial v}{\partial z}-\left(e^{\frac{1}{W e} \delta t}-1\right) \frac{\partial v\left(t^{*}\right)}{\partial z}\right]\right\}
\end{aligned}
$$


Pode ser facilmente verificado que a solução do sistema (3.49) é dada por

$$
\begin{aligned}
X & =\frac{c_{1}}{a_{1}}-\frac{b_{1}}{a_{1}} \frac{\left(a_{2} c_{1}-a_{1} c_{2}\right)}{\left(a_{2} b_{1}-a_{1} b_{2}\right)} \\
Y & =\frac{\left(a_{2} c_{1}-a_{1} c_{2}\right)}{\left(a_{2} b_{1}-a_{1} b_{2}\right)}
\end{aligned}
$$

desde que $a_{1} \neq 0$ e $\left(a_{2} b_{1}-a_{1} b_{2}\right) \neq 0$.

Assim, as componentes do tensor não-Newtoniano nos contornos rígidos paralelos ao plano- $x y$ são obtidas usando as equações (3.50) e (3.51), para o cálculo das componentes $S^{x z(n+1)}$ e $S^{y z(n+1)}$, respectivamente. Em seguida, as componentes $S^{x x(n+1)}, S^{y y(n+1)}, S^{z z(n+1)}$ e $S^{x y(n+1)}$ são obtidas utilizando as equações (3.43)-(3.46), respectivamente. 


\subsubsection{Contornos rígidos paralelos ao plano- $-x z$}

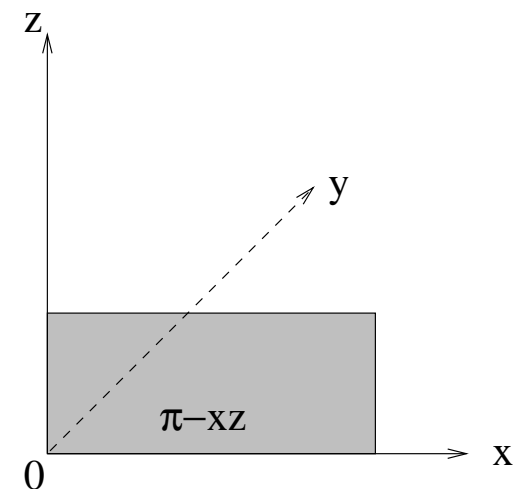

Figura 3.3: Plano $-x z(\pi-x z)$.

Nesses contornos, a condição no-slip fornece:

$$
\frac{\partial}{\partial x}=\frac{\partial}{\partial z}=0 \Rightarrow \frac{\partial v}{\partial y}=0
$$

Assim, somente os termos $\frac{\partial u}{\partial y}$ e $\frac{\partial w}{\partial y}$ são não-nulos. Dessa maneira, as equações (3.19)-(3.24) reduzem-se a

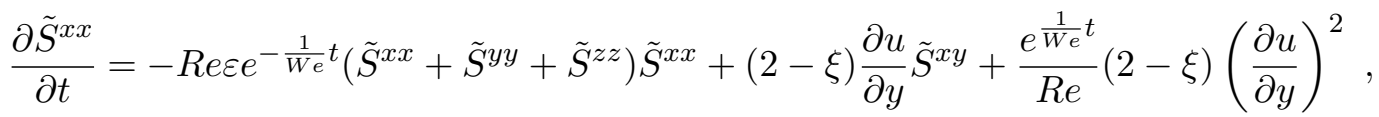

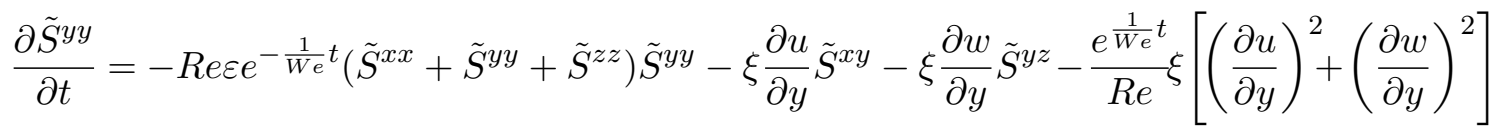

$$
\begin{aligned}
& \frac{\partial \tilde{S}^{z z}}{\partial t}=-\operatorname{Re} \varepsilon e^{-\frac{1}{W e} t}\left(\tilde{S}^{x x}+\tilde{S}^{y y}+\tilde{S}^{z z}\right) \tilde{S}^{z z}+(2-\xi) \frac{\partial w}{\partial y} \tilde{S}^{y z}+\frac{e^{\frac{1}{W e} t}}{\operatorname{Re}}(2-\xi)\left(\frac{\partial w}{\partial y}\right)^{2},
\end{aligned}
$$

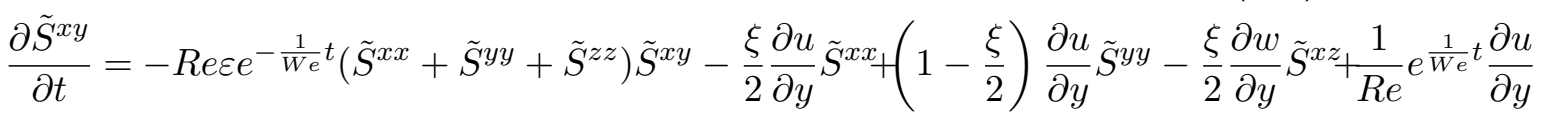

$$
\begin{aligned}
& +\frac{1}{R e} e^{\frac{1}{W e} t} \frac{\partial u}{\partial y}-\varepsilon \frac{\partial u}{\partial y}\left(\tilde{S}^{x x}+\tilde{S}^{y y}+\tilde{S}^{z z}\right)-\frac{e^{\frac{1}{W e} t}}{R e} \frac{\partial}{\partial t}\left(\frac{\partial u}{\partial y}\right),
\end{aligned}
$$

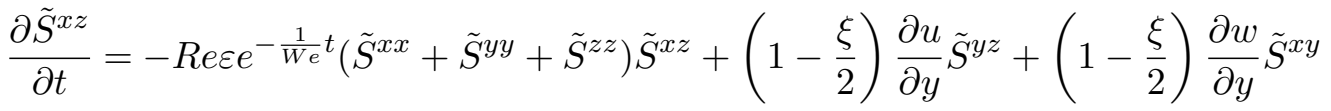

$$
\begin{aligned}
& +\frac{e^{\frac{1}{W e} t}}{R e}(2-\xi) \frac{\partial u}{\partial y} \frac{\partial w}{\partial y}
\end{aligned}
$$

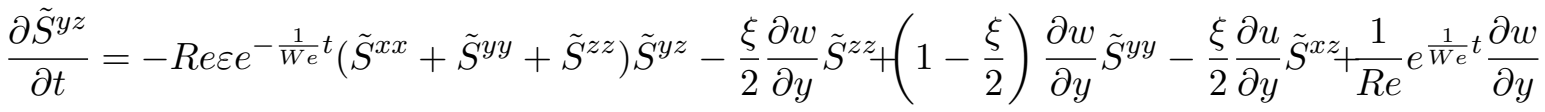

$$
\begin{aligned}
& +\frac{1}{R e} e^{\frac{1}{W e} t} \frac{\partial w}{\partial y}-\varepsilon \frac{\partial w}{\partial y}\left(\tilde{S}^{x x}+\tilde{S}^{y y}+\tilde{S}^{z z}\right)-\frac{e^{\frac{1}{W e} t}}{R e} \frac{\partial}{\partial t}\left(\frac{\partial w}{\partial y}\right) .
\end{aligned}
$$

As equaọes (3.52)-(3.57) formam um sistema $6 \times 6$ para as incógnitas $\tilde{S}^{x x}, \tilde{S}^{y y}, \tilde{S}^{z z}, \tilde{S}^{x y}, \tilde{S}^{x z}, \tilde{S}^{y z}$. Para resolvermos esse sistema, procedemos de maneira análoga ao procedimento empregado 
para calcular as componentes do tensor não-Newtoniano em contornos rígidos paralelos ao plano- $x y$. Para detalhes, ver seção 3.4.1.

\subsubsection{Contornos rígidos paralelos ao plano-yz}

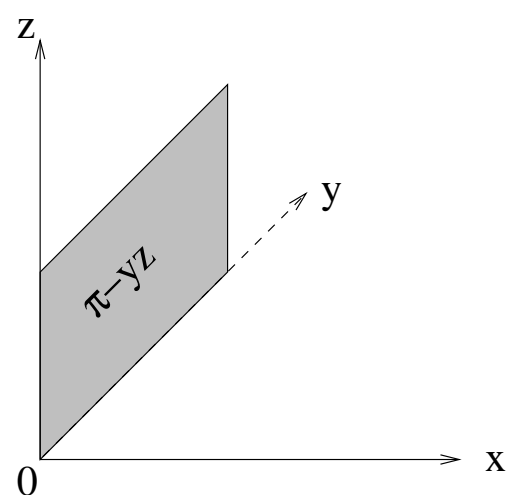

Figura 3.4: Plano-yz $(\pi-y z)$.

Nesses contornos, a condição não-escorregamentp fornece

$$
\frac{\partial}{\partial y}=\frac{\partial}{\partial z}=0 \Rightarrow \frac{\partial u}{\partial x}=0
$$

Assim, somente os termos $\frac{\partial v}{\partial x}$ e $\frac{\partial w}{\partial x}$ são não-nulos. Logo, as equações (3.19)-(3.24) reduzem-se a

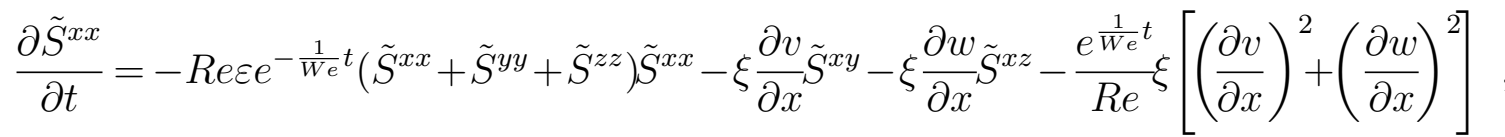

$$
\begin{aligned}
& \frac{\partial \tilde{S}^{y y}}{\partial t}=-\operatorname{Re} \varepsilon e^{-\frac{1}{W e} t}\left(\tilde{S}^{x x}+\tilde{S}^{y y}+\tilde{S}^{z z}\right) \tilde{S}^{y y}+(2-\xi) \frac{\partial v}{\partial x} \tilde{S}^{x y}+\frac{e^{\frac{1}{W e} t}}{R e}(2-\xi)\left(\frac{\partial v}{\partial x}\right)^{2} \\
& \frac{\partial \tilde{S}^{z z}}{\partial t}=-R e \varepsilon e^{-\frac{1}{W e} t}\left(\tilde{S}^{x x}+\tilde{S}^{y y}+\tilde{S}^{z z}\right) \tilde{S}^{z z}+(2-\xi) \frac{\partial w}{\partial x} \tilde{S}^{x z}+\frac{e^{\frac{1}{W e} t}}{R e}(2-\xi)\left(\frac{\partial w}{\partial x}\right)^{2}, \\
& \frac{\partial \tilde{S}^{x y}}{\partial t}=-\operatorname{Re} \varepsilon e^{-\frac{1}{W e} t}\left(\tilde{S}^{x x}+\tilde{S}^{y y}+\tilde{S}^{z z}\right) \tilde{S}^{x y}+\left[1-\frac{\xi}{2}\right] \frac{\partial v}{\partial x} \tilde{S}^{x x}-\frac{\xi}{2}\left[\frac{\partial v}{\partial x} \tilde{S}^{y y}+\frac{\partial w}{\partial x} \tilde{S}^{y z}\right]+\frac{1}{R e} e^{\frac{1}{W e} t} \frac{\partial v}{\partial x} \\
& +\frac{1}{R e} e^{\frac{1}{W e} t} \frac{\partial v}{\partial x}-\varepsilon \frac{\partial v}{\partial x}\left(\tilde{S}^{x x}+\tilde{S}^{y y}+\tilde{S}^{z z}\right)-\frac{e^{\frac{1}{W e} t}}{R e} \frac{\partial}{\partial t}\left(\frac{\partial v}{\partial x}\right), \\
& \frac{\partial \tilde{S}^{x z}}{\partial t}=-\operatorname{Re} \varepsilon e^{-\frac{1}{W e} t}\left(\tilde{S}^{x x}+\tilde{S}^{y y}+\tilde{S}^{z z}\right) \tilde{S}^{x z}+\left[1-\frac{\xi}{2}\right] \frac{\partial w}{\partial x} \tilde{S}^{x x}-\frac{\xi}{2}\left[\frac{\partial w}{\partial x} \tilde{S}^{z z}+\frac{\partial v}{\partial x} \tilde{S}^{y z}\right]+\frac{1}{R e} e^{\frac{1}{W e} t} \frac{\partial w}{\partial x} \\
& +\frac{1}{R e} e^{\frac{1}{W e} t} \frac{\partial w}{\partial x}-\varepsilon \frac{\partial w}{\partial x}\left(\tilde{S}^{x x}+\tilde{S}^{y y}+\tilde{S}^{z z}\right)-\frac{e^{\frac{1}{W e} t}}{R e} \frac{\partial}{\partial t}\left(\frac{\partial w}{\partial x}\right)
\end{aligned}
$$

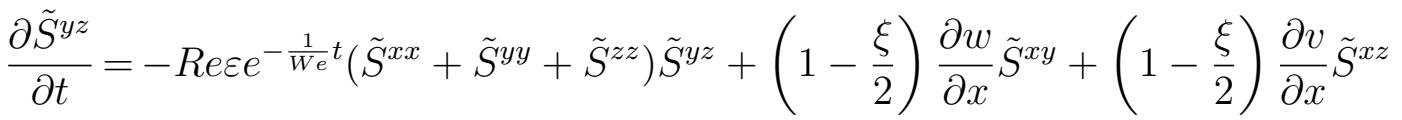

$$
\begin{aligned}
& +\frac{e^{\frac{1}{W e} t}}{R e}(2-\xi) \frac{\partial v}{\partial x} \frac{\partial w}{\partial x} .
\end{aligned}
$$


As equações(3.63)-(3.63) formam um sistema para o cálculo das componentes do tensor não-Newtoniano $S_{i j}$. Esse sistema é resolvido usando as idéias apresentadas na seção 3.4.1.

\subsection{Método de solução}

Para resolver as equações (3.6)-(3.14) empregamos o mesmo procedimento apresentado no capítulo 3 para simular escoamentos bidimensionais, o qual, para maior clareza, apresentamos novamente a seguir.

Assumimos que num dado instante de tempo $t_{n}$ o vetor velocidade $u_{i}\left(x_{k}, t_{n}\right)$ e o tensor não-Newtoniano $S_{i j}\left(x_{k}, t_{n}\right)$ são conhecidos e são dadas as condições de contorno para a velocidade e a pressão. Para calcular o vetor velocidade, o campo de pressão e o tensor não-Newtoniano no tempo $t_{n+1}=t_{n}+\delta t$, procedemos como segue:

Passo 1: Seja $\tilde{p}\left(x_{k}, t_{n}\right)$ um campo de pressão arbitrário que satisfaz a condição (3.15) na superfície livre.

Passo 3: Calcular o campo de velocidade intermediário $\widetilde{u}_{i}\left(x_{k}, t_{n+1}\right)$ por meio de

$$
\frac{\partial \widetilde{u}_{i}}{\partial t}=\frac{\partial\left(u_{k} u_{i}\right)}{\partial x_{k}}-\frac{\partial \widetilde{p}}{\partial x_{i}}+\frac{1}{R e} \frac{\partial}{\partial x_{k}}\left(\frac{\partial u_{i}}{\partial x_{k}}\right)+\frac{\partial S_{i k}}{\partial x_{k}}+\frac{1}{F r^{2}} g_{i}
$$

com $\widetilde{u}_{i}\left(x_{k}, t_{n}\right)=u_{i}\left(x_{k}, t_{n}\right)$ e utilizando as mesmas condições de contorno da velocidade $u_{i}\left(x_{k}, t_{n}\right)$. Pode-se mostrar que $\widetilde{u}_{i}\left(x_{k}, t_{n+1}\right)$ tem a vorticidade correta no tempo $t_{n+1}$.

Passo 4: Resolver a equação de Poisson

$$
\nabla^{2} \psi\left(x_{k}, t_{n+1}\right)=\nabla \cdot \widetilde{u}_{i}\left(x_{k}, t_{n+1}\right)
$$

Passo 5: Calcular a velocidade final dada por

$$
u_{i}\left(x_{k}, t_{n+1}\right)=\widetilde{u}_{i}\left(x_{k}, t_{n+1}\right)-\frac{\partial \psi\left(x_{k}, t_{n+1}\right)}{\partial x_{i}}
$$

Passo 6: Calcular a pressão:

$$
p\left(x_{k}, t_{n+1}\right)=\tilde{p}\left(x_{k}, t_{n}\right)+\frac{\psi\left(x_{k}, t_{n+1}\right)}{\delta t} .
$$

Passo 7: Calcular o tensor não-Newtoniano $S_{i j}\left(x_{k}, t_{n+1}\right)$

(a) calcular o tensor não-Newtoniano nos contornos rigídos, injetor e ejetor, conforme especificado na Seção 4.

(b) calcule $S_{i j}\left(x_{k}, t_{n+1}\right)$ por meio das equações (3.9)-(3.14). 
Passo 8: Atualizar as posições das partículas marcadoras resolvendo a equação

$$
\frac{d x_{i}}{d t}=u_{i}
$$

para cada partícula marcadora. A superfície livre do fluido é representada por uma superfície linear por partes composta por triângulos e quadriláteros em cujos vértices estão as partículas marcadoras.

\subsection{Aproximação por diferenças finitas}

Para resolver as equações que descrevem o método numérico apresentado na seção 3.5 vamos utilizar o método de diferenças finitas numa malha deslocada tridimensional. A célula computacional adotada tem dimensões $\delta x \times \delta y \times \delta z$ e as componentes do vetor velocidade estão localizados nas faces da célula enquanto que a pressão $p$, a função potencial $\psi$, o tensor não-Newtoniano e o tensor taxa de deformação são calculados no centro da célula. A figura 3.5 ilustra o tipo de célula utilizada em GENSMAC3D-PTT. Os tipos de células na malha são os

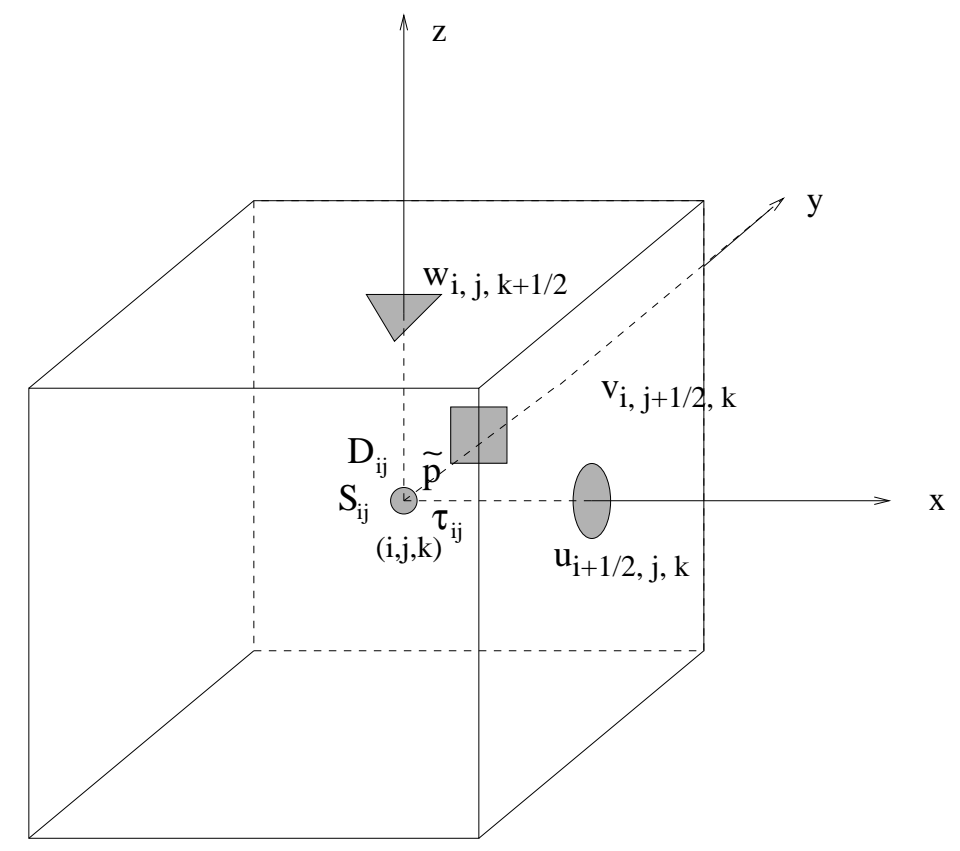

Figura 3.5: Célula tridimensional utilizada em GENSMAC3D-PTT.

mesmos utilizados em GENSMAC2D-PTT, ou seja,

$\triangleright$ células vazias (E): são células que não contém fluido;

$\triangleright$ células cheias $(\mathrm{F})$ : são células cheias de fluido e não possuem nenhuma face em contato com células vazias;

$\triangleright$ células de superfíie $(\mathrm{S})$ : são células que contém fluido e possuem pelo menos uma face em contato com células vazias; 
$\triangleright$ células de contorno rígido (B): são células que definem um contorno rígido de maneira que as condições de contorno possam ser impostas;

$\triangleright$ células de injetor (I): são células que definem a entrada de fluido;

$\triangleright$ células de ejetor $(\mathrm{O})$ : são células que definem a saía de fluido.

\subsubsection{Aproximação das equações básicas}

No cálculo das componentes da velocidade intermediária $\widetilde{u}_{i}$ a derivada temporal na equação (3.64) é aproximada pelo método de Euler explicito. O gradiente de pressão e os termos lineares são aproximados por diferenças centrais enquanto os termos convectivos são aproximados pelo método 'upwind' de alta ordem CUBISTA [1]. Os termos envolvendo o divergente do tensor não-Newtoniano são aproximados por diferenças centrais. Logo, as componentes da velocidade intermediária (3.64) são calculados pelas equações:

$$
\begin{aligned}
& \tilde{u}_{i+\frac{1}{2}, j, k}^{(n+1)}=\tilde{u}_{i+\frac{1}{2}, j, k}+\delta t\left[-\operatorname{conv}(u u)-\operatorname{conv}(v u)-\operatorname{conv}(w u)-\frac{\tilde{p}_{i+1, j, k}-\tilde{p}_{i, j, k}}{\delta x}\right. \\
& +\frac{1}{R e}\left(\frac{u_{i+\frac{3}{2}, j, k}-2 u_{i+\frac{1}{2}, j, k}+u_{i-\frac{1}{2}, j, k}}{\delta x^{2}}+\frac{u_{i+\frac{1}{2}, j+1, k}-2 u_{i+\frac{1}{2}, j, k}+u_{i+\frac{1}{2}, j-1, k}}{\delta y^{2}}\right. \\
& \left.+\frac{u_{i+\frac{1}{2}, j, k+1}-2 u_{i+\frac{1}{2}, j, k}+u_{i+\frac{1}{2}, j, k-1}}{\delta z^{2}}\right)+\frac{S_{i+1, j, k}^{x x}-S_{i, j, k}^{x x}}{\delta x} \\
& \left.+\frac{S_{i+\frac{1}{2}, j+\frac{1}{2}, k}^{x y}-S_{i+\frac{1}{2}, j-\frac{1}{2}, k}^{x y}}{\delta y}+\frac{S_{i+\frac{1}{2}, j, k+\frac{1}{2}}^{x z}-S_{i+\frac{1}{2}, j, k-\frac{1}{2}}^{x z}}{\delta z}+\frac{1}{F r^{2}} g_{x}\right] \text {, } \\
& \tilde{v}_{i, j+\frac{1}{2}, k}^{(n+1)}=\tilde{v}_{i, j+\frac{1}{2}, k}+\delta t\left[-\operatorname{conv}(u v)-\operatorname{conv}(v v)-\operatorname{conv}(w v)-\frac{\tilde{p}_{i, j+1, k}-\tilde{p}_{i, j, k}}{\delta y}\right. \\
& +\frac{1}{R e}\left(\frac{v_{i+1, j+\frac{1}{2}, k}-2 v_{i, j+\frac{1}{2}, k}+v_{i-1, j+\frac{1}{2}, k}}{\delta x^{2}}+\frac{v_{i, j+\frac{3}{2}, k}-2 v_{i, j+\frac{1}{2}, k}+v_{i, j-\frac{1}{2}, k}}{\delta y^{2}}\right. \\
& \left.+\frac{v_{i, j+\frac{1}{2}, k+1}-2 v_{i, j+\frac{1}{2}, k}+v_{i, j+\frac{1}{2}, k-1}}{\delta z^{2}}\right)+\frac{S_{i+\frac{1}{2}, j+\frac{1}{2}, k}^{x y}-S_{i-\frac{1}{2}, j+\frac{1}{2}, k}^{x y}}{\delta x} \\
& \left.+\frac{S_{i, j+1, k}^{y y}-S_{i, j, k}^{y y}}{\delta y}+\frac{S_{i, j+\frac{1}{2}, k+\frac{1}{2}}^{y z}-S_{i, j+\frac{1}{2}, k-\frac{1}{2}}^{y z}}{\delta z}+\frac{1}{F r^{2}} g_{y}\right], \\
& \tilde{w}_{i, j, k+\frac{1}{2}}^{(n+1)}=\tilde{w}_{i, j, k+\frac{1}{2}}+\delta t\left[-\operatorname{conv}(u w)-\operatorname{conv}(v w)-\operatorname{conv}(w w)-\frac{\tilde{p}_{i, j, k+1}-\tilde{p}_{i, j, k}}{\delta z}\right. \\
& +\frac{1}{R e}\left(\frac{w_{i+1, j, k+\frac{1}{2}}-2 w_{i, j, k+\frac{1}{2}}+w_{i-1, j, k+\frac{1}{2}}}{\delta x^{2}}+\frac{w_{i, j+1, k+\frac{1}{2}}-2 w_{i, j, k+\frac{1}{2}}+w_{i, j-1, k+\frac{1}{2}}}{\delta y^{2}}\right. \\
& \left.+\frac{w_{i, j, k+1}-2 w_{i, j, k+\frac{1}{2}}+w_{i, j, k-1}}{\delta z^{2}}\right)+\frac{S_{i+\frac{1}{2}, j, k+\frac{1}{2}}^{x z}-S_{i-\frac{1}{2}, j, k+\frac{1}{2}}^{x z}}{\delta x} \\
& \left.+\frac{S_{i, j+\frac{1}{2}, k+\frac{1}{2}}^{y z}-S_{i, j-\frac{1}{2}, k+\frac{1}{2}}^{y z}}{\delta y}+\frac{S_{i, j, k+1}^{z z}-S_{i, j, k}^{z z}}{\delta z}+\frac{1}{F r^{2}} g_{z}\right],
\end{aligned}
$$

onde os termos $\operatorname{conv}(\cdot)$ representam os termos convectivos e estes são discretizados pelo método CUBISTA. Nas equações acima, termos como por exemplo, $S_{i+\frac{1}{2}, j+\frac{1}{2}, k}^{x y}$ são obtidos pela média 
dos quatro valores mais próximos como segue:

$$
S_{i+\frac{1}{2}, j+\frac{1}{2}, k}^{x y}:=\frac{S_{i, j, k}^{x y}+S_{i+1, j, k}^{x y}+S_{i, j+1, k}^{x y}+S_{i+1, j+1, k}^{x y}}{4} .
$$

A equação de Poisson (3.65) é discretizada no centro da célula usando o Laplaciano de sete pontos

$$
\frac{\psi_{i+1, j, k}-2 \psi_{i, j, k}+\psi_{i-1, j, k}}{\delta x^{2}}+\frac{\psi_{i, j+1, k}-2 \psi_{i, j, k}+\psi_{i, j-1, k}}{\delta y^{2}}+\frac{\psi_{i, j, k+1}-2 \psi_{i, j, k}+\psi_{i, j, k-1}}{\delta z^{2}}=\tilde{D}_{i, j, k}
$$

onde

$$
\tilde{D}_{i, j, k}=\frac{\tilde{u}_{i+\frac{1}{2}, j, k}-\tilde{u}_{i-\frac{1}{2}, j, k}}{\delta x}+\frac{\tilde{v}_{i, j+\frac{1}{2}, k}-\tilde{v}_{i, j-\frac{1}{2}, k}}{\delta y}+\frac{\tilde{w}_{i, j, k+\frac{1}{2}}-\tilde{w}_{i, j, k-\frac{1}{2}}}{\delta z} .
$$

A equação (3.73) conduz à um sistema linear para $\psi_{i, j}$, cuja matriz associada é simétrica e positiva definida. Para resolver este sistema linear empregamos o método dos gradientes conjugados.

As componentes da velocidade, $u, v$ e $w$, no tempo $t_{n+1}$ são obtidas discretizando a equação (3.66) nos seus respectivos nós,

$$
\left\{\begin{array}{l}
u_{i+\frac{1}{2}, j, k}^{n+1}=\tilde{u}_{i+\frac{1}{2}, j, k}-\left(\frac{\psi_{i+1, j, k}-\psi_{i, j, k}}{\delta x}\right) \\
v_{i, j+\frac{1}{2}, k}^{n+1}=\tilde{v}_{i, j+\frac{1}{2}, k}-\left(\frac{\psi_{i, j+1, k}-\psi_{i, j, k}}{\delta y}\right) \\
w_{i, j, k+\frac{1}{2}}^{n+1}=\tilde{v}_{i, j, k+\frac{1}{2}}-\left(\frac{\psi_{i, j, k+1}-\psi_{i, j, k}}{\delta z}\right)
\end{array}\right.
$$

e a pressão $p$ é dada por:

$$
p_{i, j, k}=\tilde{p}_{i, j, k}+\frac{\psi_{i, j, k}}{\delta t}
$$

As componentes do tensor não-Newtoniano, dadas pelas equações (3.9) - (3.14), são aproximadas por diferenças finitas da seguinte forma: as derivadas temporais são aproximadas pelo método de Euler explícito, os termos convectivos são calculados usando o método 'upwind' de alta ordem "CUBISTA" e as derivadas espacias de primeira e segunda ordem são aproximadas por 
diferenças centrais. Logo, as equações (3.9)- (3.14) são aproximadas por:

$$
\begin{aligned}
& S_{i, j, k}^{x x}{ }^{(n+1)}=S_{i, j, k}^{x x}+\delta t\left\{-\left.\frac{1}{W e}\left(f\left(S_{k k}\right)\right)\right|_{i, j, k} S_{i, j, k}^{x x}-\operatorname{conv}\left(u S^{x x}\right)-\operatorname{conv}\left(v S^{x x}\right)-\operatorname{conv}\left(w S^{x x}\right)\right. \\
& +2(1-\xi) D_{i, j, k}^{x x} S_{i, j, k}^{x x}+\left[(2-\xi) \frac{u_{i, j+\frac{1}{2}, k}-u_{i, j-\frac{1}{2}, k}}{\delta y}-\xi \frac{v_{i+\frac{1}{2}, j, k}-v_{i-\frac{1}{2}, j, k}}{\delta x}\right] S_{i, j, k}^{x y} \\
& +\left[(2-\xi) \frac{u_{i, j, k+\frac{1}{2}}-u_{i, j, k-\frac{1}{2}}}{\delta z}-\xi \frac{w_{i+\frac{1}{2}, j, k}-w_{i-\frac{1}{2}, j, k}}{\delta x}\right] S_{i, j, k}^{x z}+\frac{2}{R e W e}\left[1-\left.f\left(S_{k k}\right)\right|_{i, j, k}\right] D_{i, j, k}^{x x} \\
& -\frac{2}{R e}\left(\frac{D_{i, j, k}^{x x}(n+1)-D_{i, j, k}^{x x}}{\delta t}+\operatorname{conv}\left(u D^{x x}\right)+\operatorname{conv}\left(v D^{x x}\right)+\operatorname{conv}\left(w D^{x x}\right)\right. \\
& -2(1-\xi)\left(D_{i, j, k}^{x x}\right)^{2}-\left[(2-\xi) \frac{u_{i, j+\frac{1}{2}, k}-u_{i, j-\frac{1}{2}, k}}{\delta y}-\xi \frac{v_{i+\frac{1}{2}, j, k}-v_{i-\frac{1}{2}, j, k}}{\delta x}\right] D_{i, j, k}^{x y} \\
& \left.\left.-\left[(2-\xi) \frac{u_{i, j, k+\frac{1}{2}}-u_{i, j, k-\frac{1}{2}}}{\delta z}-\xi \frac{w_{i+\frac{1}{2}, j, k}-w_{i-\frac{1}{2}, j, k}}{\delta x}\right] D_{i, j, k}^{x z}\right)\right\},
\end{aligned}
$$

$$
\begin{aligned}
& S_{i, j, k}^{y y}{ }^{(n+1)}=S_{i, j, k}^{y y}+\delta t\left\{-\left.\frac{1}{W e}\left(f\left(S_{k k}\right)\right)\right|_{i, j, k} S_{i, j, k}^{y y}-\operatorname{conv}\left(u S^{y y}\right)-\operatorname{conv}\left(v S^{y y}\right)-\operatorname{conv}\left(w S^{y y}\right)\right. \\
& +2(1-\xi) D_{i, j, k}^{y y} S_{i, j, k}^{y y}+\left[(2-\xi) \frac{v_{i, j, k+\frac{1}{2}}-v_{i, j, k-\frac{1}{2}}}{\delta x}-\xi \frac{u_{i, j+\frac{1}{2}, k}-u_{i, j-\frac{1}{2}, k}}{\delta y}\right] S_{i, j, k}^{x y} \\
& +\left[(2-\xi) \frac{v_{i, j, k+\frac{1}{2}}-v_{i, j, k-\frac{1}{2}}}{\delta z}-\xi \frac{w_{i, j+\frac{1}{2}, k}-w_{i, j-\frac{1}{2}, k}}{\delta y}\right] S_{i, j, k}^{y z}+\frac{2}{R e W e}\left[1-\left.f\left(S_{k k}\right)\right|_{i, j, k}\right] D_{i, j, k}^{y y} \\
& -\frac{2}{R e}\left(\frac{D_{i, j, k}^{y y}{ }^{(n+1)}-D_{i, j, k}^{y y}}{\delta t}+\operatorname{conv}\left(u D^{y y}\right)+\operatorname{conv}\left(v D^{y y}\right)+\operatorname{conv}\left(w D^{y y}\right)\right. \\
& -2(1-\xi)\left(D_{i, j, k}^{y y}\right)^{2}-\left[(2-\xi) \frac{v_{i, j, k+\frac{1}{2}}-v_{i, j, k-\frac{1}{2}}}{\delta x}-\xi \frac{u_{i, j+\frac{1}{2}, k}-u_{i, j-\frac{1}{2}, k}}{\delta y}\right] D_{i, j, k}^{x y} \\
& \left.\left.-\left[(2-\xi) \frac{v_{i, j, k+\frac{1}{2}}-v_{i, j, k-\frac{1}{2}}}{\delta z}-\xi \frac{w_{i, j+\frac{1}{2}, k}-w_{i, j-\frac{1}{2}, k}}{\delta y}\right] D_{i, j, k}^{y z}\right)\right\},
\end{aligned}
$$

$$
\begin{aligned}
& S_{i, j, k}^{z z}{ }^{(n+1)}=S_{i, j, k}^{z z}+\delta t\left\{-\left.\frac{1}{W e}\left(f\left(S_{k k}\right)\right)\right|_{i, j, k} S_{i, j, k}^{z z}-\operatorname{conv}\left(u S^{z z}\right)-\operatorname{conv}\left(v S^{z z}\right)-\operatorname{conv}\left(w S^{z z}\right)\right. \\
& +2(1-\xi) D_{i, j, k}^{z z} S_{i, j, k}^{z z}+\left[(2-\xi) \frac{w_{i+\frac{1}{2}, j, k}-w_{i-\frac{1}{2}, j, k}}{\delta x}-\xi \frac{u_{i, j, k+\frac{1}{2}}-u_{i, j, k-\frac{1}{2}}}{\delta z}\right] S_{i, j, k}^{x z} \\
& +\left[(2-\xi) \frac{w_{i, j+\frac{1}{2}, k}-w_{i, j-\frac{1}{2}, k}}{\delta y}-\xi \frac{v_{i, j, k+\frac{1}{2}}-v_{i, j, k-\frac{1}{2}}}{\delta z}\right] S_{i, j, k}^{y z}+\frac{2}{R e W e}\left[1-\left.f\left(S_{k k}\right)\right|_{i, j, k}\right] D_{i, j, k}^{z z} \\
& -\frac{2}{R e}\left(\frac{D_{i, j, k}^{z z}(n+1)}{R e} D_{i, j, k}^{z z}+\operatorname{conv}\left(u D^{z z}\right)+\operatorname{conv}\left(v D^{z z}\right)+\operatorname{conv}\left(w D^{z z}\right)\right. \\
& -2(1-\xi)\left(D_{i, j, k}^{z z}\right)^{2}-\left[(2-\xi) \frac{w_{i+\frac{1}{2}, j, k}-w_{i-\frac{1}{2}, j, k}}{\delta x}-\xi \frac{u_{i, j, k+\frac{1}{2}}-u_{i, j, k-\frac{1}{2}}}{\delta z}\right] D_{i, j, k}^{x z} \\
& \left.\left.-\left[(2-\xi) \frac{w_{i, j+\frac{1}{2}, k}-w_{i, j-\frac{1}{2}, k}}{\delta y}-\xi \frac{v_{i, j, k+\frac{1}{2}}-v_{i, j, k-\frac{1}{2}}}{\delta z}\right] D_{i, j, k}^{y z}\right)\right\},
\end{aligned}
$$




$$
\begin{aligned}
S_{i, j, k}^{x y}{ }^{(n+1)}=S_{i, j, k}^{x y}+\delta t & -\left.\frac{1}{W e}\left(f\left(S_{k k}\right)\right)\right|_{i, j, k} S_{i, j, k}^{x y}-\operatorname{conv}\left(u S^{x y}\right)-\operatorname{conv}\left(v S^{x y}\right)-\operatorname{conv}\left(w S^{x y}\right) \\
+ & (1-\xi)\left(D_{i, j, k}^{x x}+D_{i, j, k}^{y y}\right) S_{i, j, k}^{x y}+\left[\left(1-\frac{\xi}{2}\right) \frac{v_{i+\frac{1}{2}, j, k}-v_{i-\frac{1}{2}, j, k}}{\delta x}-\frac{\xi}{2} \frac{\left.u_{i, j+\frac{1}{2}, k}-u_{i, j-\frac{1}{2}, k}\right]}{\delta y}\right] S_{i, j, k}^{x x} \\
+ & {\left[\left(1-\frac{\xi}{2}\right) \frac{u_{i, j+\frac{1}{2}, k}-u_{i, j-\frac{1}{2}, k}}{\delta y}-\frac{\xi}{2} \frac{v_{i+\frac{1}{2}, j, k}-v_{i-\frac{1}{2}, j, k}}{\delta x}\right] S_{i, j, k}^{y y} } \\
+ & {\left[\left(1-\frac{\xi}{2}\right) \frac{v_{i, j, k+\frac{1}{2}}-v_{i, j, k-\frac{1}{2}}}{\delta z}-\frac{\xi}{2} \frac{w_{i, j+\frac{1}{2}, k}-w_{i, j-\frac{1}{2}, k}}{\delta y}\right] S_{i, j, k}^{x z} } \\
+ & {\left[\left(1-\frac{\xi}{2}\right) \frac{u_{i, j, k+\frac{1}{2}}-u_{i, j, k-\frac{1}{2}}}{\delta z}-\frac{\xi}{2} \frac{w_{i+\frac{1}{2}, j, k}-w_{i-\frac{1}{2}, j, k}}{\delta x}\right] S_{i, j, k}^{y z}+\frac{2}{R e W e}\left[1-\left.f\left(S_{k k}\right)\right|_{i, j, k}\right] D_{i, j, k}^{x y} } \\
- & \frac{2}{R e}\left(\frac{D_{i, j, k}^{x y}(n+1)-D_{i, j, k}^{x y}+\mathbf{c o n v}\left(u D^{x y}\right)+\operatorname{conv}\left(v D^{x y}\right)+\operatorname{conv}\left(w D^{x y}\right)}{\delta t}\right] \\
- & (1-\xi)\left(D_{i, j, k}^{x x}+D_{i, j, k}^{y y}\right) D_{i, j, k}^{x y}-\left[\left(1-\frac{\xi}{2}\right) \frac{v_{i+\frac{1}{2}, j, k}-v_{i-\frac{1}{2}, j, k}}{\delta x}-\frac{\xi}{2} \frac{u_{i, j+\frac{1}{2}, k}-u_{i, j-\frac{1}{2}, k}}{\delta y}\right] D_{i, j, k}^{x x} \\
- & {\left[\left(1-\frac{\xi}{2}\right) \frac{u_{i, j+\frac{1}{2}, k}-u_{i, j-\frac{1}{2}, k}}{\delta y}-\frac{\xi}{2} \frac{v_{i+\frac{1}{2}, j, k}-v_{i-\frac{1}{2}, j, k}}{\delta x}\right] D_{i, j, k}^{y y}-\left[\left(1-\frac{\xi}{2}\right) \frac{v_{i, j, k+\frac{1}{2}}-v_{i, j, k-\frac{1}{2}}}{\delta z}\right.} \\
& \left.\left.-\frac{\xi}{2} \frac{w_{i, j+\frac{1}{2}, k}-w_{i, j-\frac{1}{2}, k}}{\delta y}\right] D^{x z}-\left[\left(1-\frac{\xi}{2}\right) \frac{u_{i, j, k+\frac{1}{2}}-u_{i, j, k-\frac{1}{2}}}{\delta z}-\frac{\xi w_{i+\frac{1}{2}, j, k}-w_{i-\frac{1}{2}, j, k}}{2} D_{i, j, k}^{y z}\right)\right\}
\end{aligned}
$$

$S_{i, j, k}^{x z}{ }^{(n+1)}=S_{i, j, k}^{x z}+\delta t\left\{-\left.\frac{1}{W e}\left(f\left(S_{k k}\right)\right)\right|_{i, j, k} S_{i, j, k}^{x z}-\operatorname{conv}\left(u S^{x z}\right)-\operatorname{conv}\left(v S^{x z}\right)-\operatorname{conv}\left(w S^{x z}\right)\right.$

$+(1-\xi)\left(D_{i, j, k}^{x x}+D_{i, j, k}^{z z}\right) S_{i, j, k}^{x z}+\left[\left(1-\frac{\xi}{2}\right) \frac{w_{i+\frac{1}{2}, j, k}-w_{i-\frac{1}{2}, j, k}}{\delta x}-\frac{\xi}{2} \frac{u_{i, j, k+\frac{1}{2}}-u_{i, j, k-\frac{1}{2}}}{\delta z}\right] S_{i, j, k}^{x x}$

$+\left[\left(1-\frac{\xi}{2}\right) \frac{u_{i, j, k+\frac{1}{2}}-u_{i, j, k-\frac{1}{2}}}{\delta z}-\frac{\xi}{2} \frac{w_{i+\frac{1}{2}, j, k}-w_{i-\frac{1}{2}, j, k}}{\delta x}\right] S_{i, j, k}^{z z}$

$+\left[\left(1-\frac{\xi}{2}\right) \frac{w_{i, j+\frac{1}{2}, k}-w_{i, j-\frac{1}{2}, k}}{\delta y}-\frac{\xi}{2} \frac{v_{i, j, k+\frac{1}{2}}-v_{i, j, k-\frac{1}{2}}}{\delta z}\right] S_{i, j, k}^{x y}$

$+\left[\left(1-\frac{\xi}{2}\right) \frac{u_{i, j+\frac{1}{2}, k}-u_{i, j-\frac{1}{2}, k}}{\delta y}-\frac{\xi}{2} \frac{v_{i+\frac{1}{2}, j, k}-v_{i-\frac{1}{2}, j, k}}{\delta x}\right] S_{i, j, k}^{y z}+\frac{2}{R e W e}\left[1-\left.f\left(S_{k k}\right)\right|_{i, j, k}\right] D_{i, j, k}^{x z}$

$-\frac{2}{R e}\left(\frac{D_{i, j, k}^{x z}{ }^{(n+1)}-D_{i, j, k}^{x z}}{\delta t}+\operatorname{conv}\left(u D^{x z}\right)+\operatorname{conv}\left(v D^{x z}\right)+\operatorname{conv}\left(w D^{x z}\right)\right.$

$-(1-\xi)\left(D_{i, j, k}^{x x}+D_{i, j, k}^{z z}\right) D_{i, j, k}^{x z}-\left[\left(1-\frac{\xi}{2}\right) \frac{w_{i+\frac{1}{2}, j, k}-w_{i-\frac{1}{2}, j, k}}{\delta x}-\frac{\xi}{2} \frac{u_{i, j, k+\frac{1}{2}}-u_{i, j, k-\frac{1}{2}}}{\delta z}\right] D_{i, j, k}^{x x}$

$-\left[\left(1-\frac{\xi}{2}\right) \frac{u_{i, j, k+\frac{1}{2}}-u_{i, j, k-\frac{1}{2}}}{\delta z}-\frac{\xi}{2} \frac{w_{i+\frac{1}{2}, j, k}-w_{i-\frac{1}{2}, j, k}}{\delta x}\right] D_{i, j, k}^{z z}-\left[\left(1-\frac{\xi}{2}\right) \frac{w_{i, j+\frac{1}{2}, k}-w_{i, j-\frac{1}{2}, k}}{\delta y}\right.$

$\left.\left.\left.-\frac{\xi}{2} \frac{v_{i, j, k+\frac{1}{2}}-v_{i, j, k-\frac{1}{2}}}{\delta z}\right] D_{i, j, k}^{x y}-\left[\left(1-\frac{\xi}{2}\right) \frac{u_{i, j+\frac{1}{2}, k}-u_{i, j-\frac{1}{2}, k}}{\delta y}-\frac{\xi}{2} \frac{v_{i+\frac{1}{2}, j, k}-v_{i-\frac{1}{2}, j, k}}{\delta x}\right] D_{i, j, k}^{y z}\right)\right\}$, 


$$
\begin{aligned}
S_{i, j, k}^{y z}(n+1) & =S_{i, j, k}^{y z}+\delta t\left\{-\left.\frac{1}{W e}\left(f\left(S_{k k}\right)\right)\right|_{i, j, k} S_{i, j, k}^{y z}-\operatorname{conv}\left(u S^{y z}\right)-\operatorname{conv}\left(v S^{y z}\right)-\operatorname{conv}\left(w S^{y z}\right)\right. \\
+ & (1-\xi)\left(D_{i, j, k}^{y y}+D_{i, j, k}^{z z}\right) S_{i, j, k}^{y z}+\left[\left(1-\frac{\xi}{2}\right) \frac{w_{i, j+\frac{1}{2}, k}-w_{i, j-\frac{1}{2}, k}}{\delta y}-\frac{\xi}{2} \frac{v_{i, j, k+\frac{1}{2}}-v_{i, j, k-\frac{1}{2}}}{\delta z}\right] S_{i, j, k}^{y y} \\
+ & {\left[\left(1-\frac{\xi}{2}\right) \frac{v_{i, j, k+\frac{1}{2}}-v_{i, j, k-\frac{1}{2}}}{\delta z}-\frac{\xi}{2} \frac{w_{i, j+\frac{1}{2}, k}-w_{i, j-\frac{1}{2}, k}}{\delta y}\right] S_{i, j, k}^{z z} } \\
+ & {\left[\left(1-\frac{\xi}{2}\right) \frac{w_{i+\frac{1}{2}, k}, j-w_{i-\frac{1}{2}, j, k}}{\delta x}-\frac{\xi}{2} \frac{u_{i, j, k+\frac{1}{2}}-u_{i, j, k-\frac{1}{2}}}{\delta z}\right] S_{i, j, k}^{x y} } \\
+ & {\left[\left(1-\frac{\xi}{2}\right) \frac{v_{i+\frac{1}{2}, j, k}-v_{i-\frac{1}{2}, j, k}}{\delta x}-\frac{\xi}{2} \frac{u_{i, j+\frac{1}{2}, k}-u_{i, j-\frac{1}{2}, k}}{\delta y}\right] S_{i, j, k}^{x z}+\frac{2}{R e W e}\left[1-\left.f\left(S_{k k}\right)\right|_{i, j, k}\right] D_{i, j, k}^{y z} } \\
- & \frac{2}{R e}\left(\frac{D_{i, j, k}^{y z}(n+1)-D_{i, j, k}^{y z}+\operatorname{conv}\left(u D^{y z}\right)+\operatorname{conv}\left(v D^{y z}\right)+\operatorname{conv}\left(w D^{y z}\right)}{\delta t}\right] \\
- & (1-\xi)\left(D_{i, j, k}^{y y}+D_{i, j, k}^{z z}\right) D_{i, j, k}^{y z}-\left[\left(1-\frac{\xi}{2}\right) \frac{w_{i, j+\frac{1}{2}, k}-w_{i, j-\frac{1}{2}, k}}{\delta y}-\frac{\xi}{2} \frac{v_{i, j, k+\frac{1}{2}}-v_{i, j, k-\frac{1}{2}}}{\delta z}\right] D_{i, j, k}^{y y} \\
- & {\left[\left(1-\frac{\xi}{2}\right) \frac{v_{i, j, k+\frac{1}{2}}-v_{i, j, k-\frac{1}{2}}}{\delta z}-\frac{\xi}{2} \frac{w_{i, j+\frac{1}{2}, k}-w_{i, j-\frac{1}{2}, k}}{\delta y}\right] D_{i, j, k}^{z z}-\left[\left(1-\frac{\xi}{2}\right) \frac{w_{i+\frac{1}{2}, k}, j-w_{i-\frac{1}{2}, j, k}}{\delta x}\right.} \\
- & -\frac{\xi u_{i, j, k+\frac{1}{2}}-u_{i, j, k-\frac{1}{2}}}{\delta z} D_{i, j, k}^{x y}-\left[\left(1-\frac{\xi}{2}\right) \frac{v_{i+\frac{1}{2}, j, k}-v_{i-\frac{1}{2}, j, k}}{\delta x}-\frac{\xi}{2} \frac{\left.\left.\left.u_{i, j+\frac{1}{2}, k}-u_{i, j-\frac{1}{2}, k}\right] D_{i, j, k}^{x z}\right)\right\}}{\delta y}\right.
\end{aligned}
$$

onde,

$$
\begin{array}{ll}
D_{i, j, k}^{x x}=\left(\frac{u_{i+\frac{1}{2}, j, k}-u_{i-\frac{1}{2}, j, k}}{\delta x}\right), & D_{i, j, k}^{x y}=\frac{1}{2}\left(\frac{u_{i, j+\frac{1}{2}, k}-u_{i, j-\frac{1}{2}}, k}{\delta y}+\frac{v_{i+\frac{1}{2}, j, k}-v_{i-\frac{1}{2}, j, k}}{\delta x}\right), \\
D_{i, j, k}^{y y}=\left(\frac{v_{i, j+\frac{1}{2}, k}-v_{i, j-\frac{1}{2}, k}}{\delta y}\right), & D_{i, j, k}^{x z}=\frac{1}{2}\left(\frac{u_{i, j, k+\frac{1}{2}}-u_{i, j, k-\frac{1}{2}}}{\delta z}+\frac{w_{i+\frac{1}{2}, j, k}-w_{i-\frac{1}{2}, j, k}}{\delta x}\right), \\
D_{i, j, k}^{z z}=\left(\frac{w_{i, j, k+\frac{1}{2}}-w_{i, j, k-\frac{1}{2}}}{\delta z}\right), & D_{i, j, k}^{y z}=\frac{1}{2}\left(\frac{v_{i, j, k+\frac{1}{2}}-v_{i, j, k-\frac{1}{2}}}{\delta z}+\frac{w_{i, j+\frac{1}{2}, k}-w_{i, j-\frac{1}{2}, k}}{\delta y}\right),
\end{array}
$$

e $\left.f\left(S_{k k}\right)\right|_{i, j, k}=1+\varepsilon \operatorname{ReWe}\left(S_{i, j, k}^{x x}+S_{i, j, k}^{y y}+S_{i, j, k}^{z z}\right)$.

Nas equações (3.75)-(3.80) os termos que não são avaliados nos seus respectivos nós são obtidos pela média aritmética dos nós vizinhos, por exemplo:

$$
w_{i+\frac{1}{2}, j, k}:=\frac{w_{i, j, k+\frac{1}{2}}+w_{i+1, j, k+\frac{1}{2}}+w_{i, j, k-\frac{1}{2}}+w_{i+1, j, k-\frac{1}{2}}}{4}
$$

\subsubsection{Aproximação do tensor não-Newtoniano em contornos rígidos}

Os valores de $S^{x x}, S^{y y}, S^{z z}, S^{x y}, S^{x z}$ e $S^{y z}$ nas células do contorno rígido são obtidos através das equações derivadas na Seção 3.4 as quais são discretizadas no centro das faces das células do contorno rígido (B) que estão em contato com células do interior do domínio.

A seguir consideramos a discretização em contornos paralelos ao plano-xy. Nestes contornos dois casos são considerados: células B com apenas a face superior e células B com apenas a face inferior em contato com uma célula do interior do domínio. Por exemplo, para a configuração mostrada na figura 3.6, as equações derivadas na Seção 3.4 (ver equações (3.43)-(3.46), 
(3.50) e (3.51))) são discretizadas como segue:

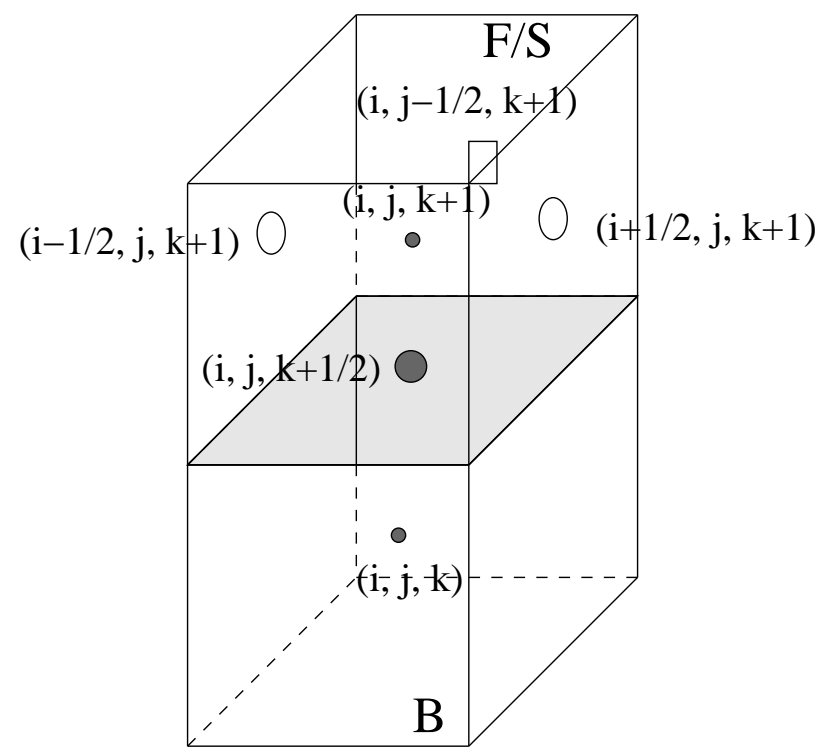

Figura 3.6: Célula do contorno rígido com a face superior em contato com uma célula do interior do domínio.

$$
\begin{aligned}
& S_{i, j, k+\frac{1}{2}}^{x x(n+1)}=e^{-\frac{1}{W e} \delta t} S_{i, j, k+\frac{1}{2}}^{x x}+(2-\xi) \frac{\delta t}{2}\left(\left.\frac{\partial u}{\partial z}\right|_{i, j, k+\frac{1}{2}} e^{-\frac{1}{W e} \delta t} S_{i, j, k+\frac{1}{2}}^{x z}+\left.\frac{\partial u^{(n+1)}}{\partial z}\right|_{i, j, k+\frac{1}{2}} S_{i, j, k+\frac{1}{2}}^{x z(n+1)}\right) \\
& +(2-\xi) \frac{W e}{R e}\left(1-e^{\frac{1}{W e} \delta t}\right)\left(\left.\frac{\partial u\left(t^{*}\right)}{\partial z}\right|_{i, j, k+\frac{1}{2}}\right)^{2} \\
& S_{i, j, k+\frac{1}{2}}^{y y(n+1)}=e^{-\frac{1}{W e} \delta t} S_{i, j, k+\frac{1}{2}}^{y y}+(2-\xi) \frac{\delta t}{2}\left(\left.\frac{\partial v}{\partial z}\right|_{i, j, k+\frac{1}{2}} e^{-\frac{1}{W e} \delta t} S_{i, j, k+\frac{1}{2}}^{y z}+\left.\frac{\partial v^{(n+1)}}{\partial z}\right|_{i, j, k+\frac{1}{2}} S_{i, j, k+\frac{1}{2}}^{y z(n+1)}\right) \\
& +(2-\xi) \frac{W e}{R e}\left(1-e^{\frac{1}{W e} \delta t}\right)\left(\left.\frac{\partial v\left(t^{*}\right)}{\partial z}\right|_{i, j, k+\frac{1}{2}}\right)^{2}, \\
& S_{i, j, k+\frac{1}{2}}^{z z(n+1)}=e^{-\frac{1}{W e} \delta t} S_{i, j, k+\frac{1}{2}}^{z z}-\xi \frac{\delta t}{2}\left(\left.\frac{\partial u}{\partial z}\right|_{i, j, k+\frac{1}{2}} e^{-\frac{1}{W e} \delta t} S_{i, j, k+\frac{1}{2}}^{x z}+\left.\frac{\partial u^{(n+1)}}{\partial z}\right|_{i, j, k+\frac{1}{2}} S_{i, j, k+\frac{1}{2}}^{x z(n+1)}\right) \\
& -\xi \frac{\delta t}{2}\left(\left.\frac{\partial v}{\partial z}\right|_{i, j, k+\frac{1}{2}} e^{-\frac{1}{W e} \delta t} S_{i, j, k+\frac{1}{2}}^{y z}+\left.\frac{\partial v^{(n+1)}}{\partial z}\right|_{i, j, k+\frac{1}{2}} S_{i, j, k+\frac{1}{2}}^{y z(n+1)}\right) \\
& -\xi \frac{W e}{R e}\left(1-e^{\frac{1}{W e} \delta t}\right)\left[\left(\left.\frac{\partial u\left(t^{*}\right)}{\partial z}\right|_{i, j, k+\frac{1}{2}}\right)^{2}+\left(\left.\frac{\partial v\left(t^{*}\right)}{\partial z}\right|_{i, j, k+\frac{1}{2}}\right)^{2}\right]
\end{aligned}
$$




$$
\begin{aligned}
S_{i, j, k+\frac{1}{2}}^{x y(n+1)}= & e^{-\frac{1}{W e} \delta t} S_{i, j, k+\frac{1}{2}}^{x y y}+\left(1-\frac{\xi}{2}\right) \frac{\delta t}{2}\left(\left.\frac{\partial u}{\partial z}\right|_{i, j, k+\frac{1}{2}} e^{-\frac{1}{W e} \delta t} S_{i, j, k+\frac{1}{2}}^{y z}+\left.\frac{\partial u^{(n+1)}}{\partial z}\right|_{i, j, k+\frac{1}{2}} S_{i, j, k+\frac{1}{2}}^{y z(n+1)}\right) \\
& +\left(1-\frac{\xi}{2}\right) \frac{\delta t}{2}\left(\left.\frac{\partial v}{\partial z}\right|_{i, j, k+\frac{1}{2}} e^{-\frac{1}{W e} \delta t} S_{i, j, k+\frac{1}{2}}^{x z}+\left.\frac{\partial v^{(n+1)}}{\partial z}\right|_{i, j, k+\frac{1}{2}} S_{i, j, k+\frac{1}{2}}^{x z(n+1)}\right) \\
& +\left.\left.(2-\xi) \frac{W e}{R e}\left(1-e^{\frac{1}{W e} \delta t}\right) \frac{\partial u\left(t^{*}\right)}{\partial z}\right|_{i, j, k+\frac{1}{2}} \frac{\partial v\left(t^{*}\right)}{\partial z}\right|_{i, j, k+\frac{1}{2}} \\
S_{\substack{x z(n+1) \\
i, j, k+\frac{1}{2}}=} & \left.\left(\frac{c_{1}}{a_{1}}-\frac{b_{1}}{a_{1}} \frac{\left(a_{2} c_{1}-a_{1} c_{2}\right)}{\left(a_{2} b_{1}-a_{1} b_{2}\right)}\right)\right|_{i, j, k+\frac{1}{2}} \\
S_{i, j, k+\frac{1}{2}}^{y z(n+1)}= & \left.\left(\frac{\left(a_{2} c_{1}-a_{1} c_{2}\right)}{\left(a_{2} b_{1}-a_{1} b_{2}\right)}\right)\right|_{i, j, k+\frac{1}{2}}
\end{aligned}
$$

desde que $\left.a_{1}\right|_{i, j, k+\frac{1}{2}} \neq 0$ e $\left.\left(a_{2} b_{1}-a_{1} b_{2}\right)\right|_{i, j, k+\frac{1}{2}} \neq 0$. As constantes $a_{1}, b_{1}, c_{1}, a_{2}, b_{2}$ e $c_{2}$ estão definidas na Seção 3.4.

Os valores de $\left.\left.\frac{\partial u\left(t^{*}\right)}{\partial z}\right|_{i, j, k+\frac{1}{2}} \mathrm{e} \frac{\partial v\left(t^{*}\right)}{\partial z}\right|_{i, j, k+\frac{1}{2}}$ são obtidos pelas médias entre os tempos $t_{n} \mathrm{e}$ $t_{n+1}$, ou seja,

$$
\begin{aligned}
& \left.\frac{\partial u\left(t^{*}\right)}{\partial z}\right|_{i, j, k+\frac{1}{2}}=\frac{1}{2}\left[\left.\frac{\partial u}{\partial z}\right|_{i, j, k+\frac{1}{2}}+\left.\frac{\partial u^{(n+1)}}{\partial z}\right|_{i, j, k+\frac{1}{2}}\right] \\
& \left.\frac{\partial v\left(t^{*}\right)}{\partial z}\right|_{i, j, k+\frac{1}{2}}=\frac{1}{2}\left[\left.\frac{\partial v}{\partial z}\right|_{i, j, k+\frac{1}{2}}+\left.\frac{\partial v^{(n+1)}}{\partial z}\right|_{i, j, k+\frac{1}{2}}\right]
\end{aligned}
$$

e essas derivadas são aproximadas como segue,

$$
\begin{array}{lll}
\left.\frac{\partial u}{\partial z}\right|_{i, j, k+\frac{1}{2}}=\frac{u_{i, j, k+1}^{n}-u_{i, j, k+\frac{1}{2}}^{n}}{\frac{\delta z}{2}}, & \left.\frac{\partial u^{(n+1)}}{\partial y}\right|_{i, j, k+\frac{1}{2}}=\frac{u_{i, j, k+1}^{(n+1)}-u_{i, j, k+\frac{1}{2}}^{(n+1)}}{\frac{\delta z}{2}} \\
\left.\frac{\partial v}{\partial z}\right|_{i, j, k+\frac{1}{2}}=\frac{v_{i, j, k+1}^{n}-v_{i, j, k+\frac{1}{2}}^{n}}{\frac{\delta z}{2}}, & \left.\frac{\partial v^{(n+1)}}{\partial z}\right|_{i, j, k+\frac{1}{2}}=\frac{v_{i, j, k+1}^{(n+1)}-v_{i, j, k+\frac{1}{2}}^{(n+1)}}{\frac{\delta z}{2}}
\end{array}
$$

As velocidades na posição $\left(i, j, k+\frac{1}{2}\right)$ são nulas pela condição de não-escorregamento e as velocidades na posição $(i, j, k+1)$ são dadas por

$$
u_{i, j, k+1}=\frac{u_{i+\frac{1}{2}, j, k+1}+u_{i-\frac{1}{2}, j, k+1}}{2} \text { e } \quad v_{i, j, k+1}=\frac{v_{i, j+\frac{1}{2}, k+1}+v_{i, j-\frac{1}{2}, k+1}}{2} \text {, respectivamente. }
$$

Finalmente, os valores de $S_{i, j, k}^{x x}, S_{i, j, k}^{y y}, S_{i, j, k}^{z z}, S_{i, j, k}^{x y}, S_{i, j, k}^{x z}$ e $S_{i, j, k}^{y z}$ são obtidos pela interpolação linear usando os nós $\left(i, j, k+\frac{1}{2}\right)$ e $(i, j, k+1)$, respectivamente. 


\subsubsection{Condição de contorno na superfície livre}

As condições de contorno na superfície livre dadas pelas equações (3.16)-(3.18) são aproximadas pelo método de diferenças finitas seguindo as idéias de Tomé et al. [80].

Supomos que a malha tenha um espaçamento suficientemente pequeno de maneira que, localmente, a superfície livre pode ser aproximada por uma superfície plana. Nesse trabalho, consideramos três tipos de superfícies planas: superfícies-1D, superfícies-2D e superfícies-3D. Superfícies-1D são as superfícies que são paralelas aos planos formados pelos eixos $x, y$ ou $x, z$ ou $z, y$ enquanto as superfícies-2D e superfícies-3D são superfícies que fazem um ângulo de $45^{\circ} \mathrm{e}$ $60^{\circ}$, com os eixos coordenados, respectivamente. (ver figura 3.7). Detalhes dessas aproximações são apresentados a seguir.

a)

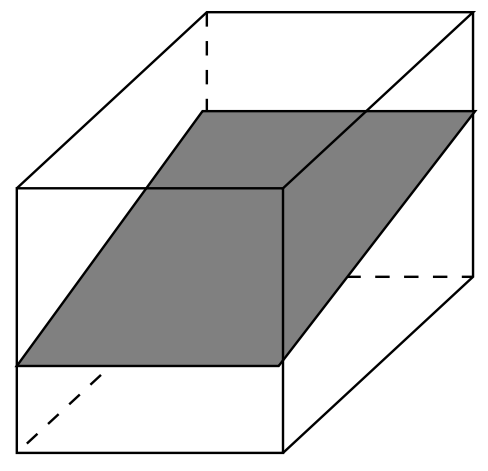

b)

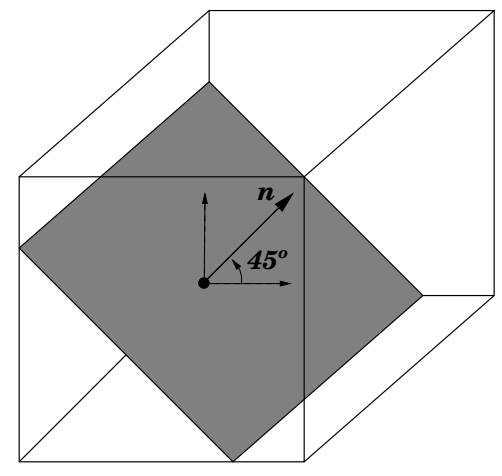

c)

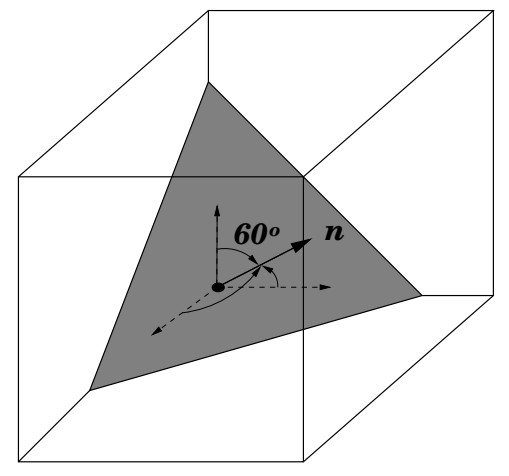

Figura 3.7: Tipos de superfícies planas. a) superfície-1D; b) superfície-2D; c) superfície-3D

i) superfícies-1D: Essas superfícies são identificadas por células de superfície (S) que possuem apenas uma face em contato com uma face de células vazias (E). Nessas superfícies,

a)

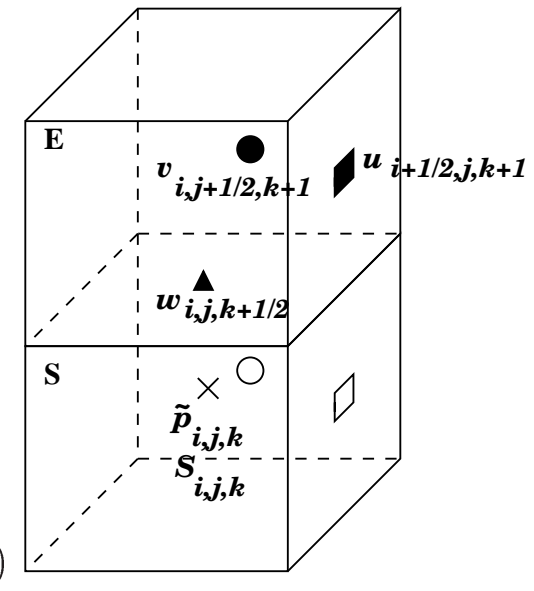

b)

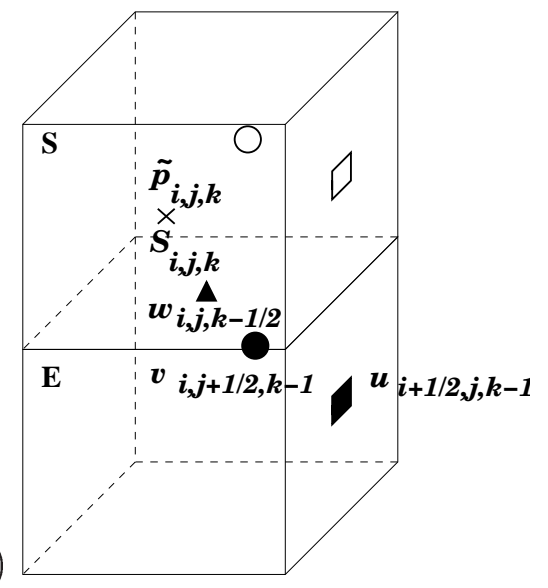

Figura 3.8: Células de superfícies $(\mathrm{S})$ com apenas uma face em contato com uma face de célula vazia (E). a) face $\left(k-\frac{1}{2}\right)$ da célula $(\mathrm{S})$ em contato com uma face da célula (E); b) face $\left(k+\frac{1}{2}\right)$ da célula $(\mathrm{S})$ em contato com uma face da célula (E).

assumimos que o vetor normal aponta para um dos eixos coordenados: $x, y$ ou $z$. Logo, 
o vetor normal pode ser escrito como: $( \pm 1,0,0),(0, \pm 1,0)$ ou $(0,0, \pm 1)$. Se uma célula de superfície tem a face $\left(k+\frac{1}{2}\right)$ ou a face $\left(k-\frac{1}{2}\right)$ em contato com uma face de uma célula vazia (ver figura 3.8) então assumimos que a superfície livre está paralela ao plano-xy e o vetor normal aponta para a direção $z$. Assim, o vetor normal tem a forma $\mathbf{n}=(0,0, \pm 1) \mathrm{e}$ os vetores tangenciais são tomados como sendo $\mathbf{m}_{\mathbf{1}}=(0,1,0)$ e $\mathbf{m}_{\mathbf{2}}=(1,0,0)$. Portanto, as condições para o contorno na superfície livre (3.16)-(3.18) reduzem-se a:

$$
\begin{gathered}
\tilde{p}=\frac{2}{R e} \frac{\partial w}{\partial z}+S^{z z}=0, \\
\frac{1}{R e}\left(\frac{\partial v}{\partial z}+\frac{\partial w}{\partial y}\right)+S^{y z}=0, \\
\frac{1}{R e}\left(\frac{\partial u}{\partial z}+\frac{\partial w}{\partial x}\right)+S^{x z}=0 .
\end{gathered}
$$

Por exemplo, adotando a configuração de célula mostrada na figura 3.8a, o valor da pressão $p_{i, j, k}$ e os valores das velocidades $w_{i, j, k+\frac{1}{2}}, u_{i+\frac{1}{2}, j, k+1}$ e $v_{i, j+\frac{1}{2}, k+1}$ são requeridos. Esses valores são obtidos como segue. Impondo conservação de massa no centro da célula de superfície obtém-se:

$$
\frac{u_{i+\frac{1}{2}, j, k}-u_{i-\frac{1}{2}, j, k}}{\delta x}+\frac{v_{i, j+\frac{1}{2}, k}-v_{i, j-\frac{1}{2}, k}}{\delta y}+\frac{w_{i, j, k+\frac{1}{2}}-w_{i, j, k-\frac{1}{2}}}{\delta z}=0 .
$$

Agora, discretizando a equação (3.90) na posição $\left(i+\frac{1}{2}, j, k+\frac{1}{2}\right)$ vem:

$$
\frac{u_{i+\frac{1}{2}, j, k+1}-u_{i+\frac{1}{2}, j, k}}{\delta z}+\frac{w_{i+1, j, k+\frac{1}{2}}-w_{i, j, k+\frac{1}{2}}}{\delta x}=\operatorname{Re} S_{i+\frac{1}{2}, j, k+\frac{1}{2}}^{x z}
$$

e de forma similar discretizando a equação (3.89) no ponto $\left(i, j+\frac{1}{2}, k+\frac{1}{2}\right)$ tem-se:

$$
\frac{v_{i, j+\frac{1}{2}, k+1}-v_{i, j+\frac{1}{2}, k}}{\delta z}+\frac{w_{i, j+1, k+\frac{1}{2}}-w_{i, j, k+\frac{1}{2}}}{\delta y}=\operatorname{Re} S_{i, j+\frac{1}{2}, k+\frac{1}{2}}^{y z} .
$$

As equações (3.91)-(3.93) constituem um sistema linear $3 \times 3$ para as incógnitas $w_{i, j, k+\frac{1}{2}}$, $u_{i+\frac{1}{2}, j, k+1}$ e $v_{i, j+\frac{1}{2}, k+1}$, que uma vez resolvido, fornece:

$$
\begin{aligned}
w_{i, j, k+\frac{1}{2}} & =w_{i, j, k-\frac{1}{2}}-\frac{\delta z}{\delta x}\left(u_{i+\frac{1}{2}, j, k}-u_{i-\frac{1}{2}, j, k}\right)-\frac{\delta z}{\delta y}\left(v_{i, j+\frac{1}{2}, k}-v_{i, j-\frac{1}{2}, k}\right) \\
u_{i+\frac{1}{2}, j, k+1} & =u_{i+\frac{1}{2}, j, k}-\frac{\delta z}{\delta x}\left(w_{i+1, j, k+\frac{1}{2}}-w_{i, j, k+\frac{1}{2}}\right)-\delta z \operatorname{Re} S_{i+\frac{1}{2}, j, k+\frac{1}{2}}^{x z} \\
v_{i, j+\frac{1}{2}, k+1} & =v_{i, j+\frac{1}{2}, k}-\frac{\delta z}{\delta y}\left(w_{i, j+1, k+\frac{1}{2}}-w_{i, j, k+\frac{1}{2}}\right)-\delta z \operatorname{Re} S_{i, j+\frac{1}{2}, k+\frac{1}{2}}^{y z}
\end{aligned}
$$

Tendo obtido as velocidades através das equações (3.94)-(3.96) podemos calcular a pressão 
discretizando a equação (3.88) no centro da célula de superfície:

$$
\tilde{p}_{i, j, k}-\frac{2}{R e} \frac{w_{i, j, k+\frac{1}{2}}-w_{i, j, k-\frac{1}{2}}}{\delta z}+S_{i, j, k}^{z z}=0 .
$$

Se a célula de superfície tem somente a face $\left(k-\frac{1}{2}\right)$ em contato com uma face de uma célula vazia (ver figura 3.8b, os valores das velocidades e pressão são obtidos de maneira análoga e são dados por:

$$
\begin{aligned}
w_{i, j, k-\frac{1}{2}} & =w_{i, j, k+\frac{1}{2}}+\frac{\delta z}{\delta x}\left(u_{i+\frac{1}{2}, j, k}-u_{i-\frac{1}{2}, j, k}\right)-\frac{\delta z}{\delta y}\left(v_{i, j+\frac{1}{2}, k}-v_{i, j-\frac{1}{2}, k}\right) \\
u_{i+\frac{1}{2}, j, k-1} & =u_{i+\frac{1}{2}, j, k}-\frac{\delta z}{\delta x}\left(w_{i+1, j, k-\frac{1}{2}}-w_{i, j, k-\frac{1}{2}}\right)-\delta z \operatorname{Re} S_{i+\frac{1}{2}, j, k-\frac{1}{2}}^{x z} \\
v_{i, j+\frac{1}{2}, k-1} & =v_{i, j+\frac{1}{2}, k}-\frac{\delta z}{\delta y}\left(w_{i, j+1, k-\frac{1}{2}}-w_{i, j, k-\frac{1}{2}}\right)-\delta z \operatorname{Re} S_{i, j+\frac{1}{2}, k-\frac{1}{2}}^{y z} .
\end{aligned}
$$

A aplicação das condições de contorno na superfície livre nos outros casos de superfícies-1D é feita de maneira análoga aos casos acima.

ii) Superfícies-2D: Estas superfícies são identificadas por células de superfície (S) que possuem duas faces adjacentes em contato com faces de células vazias (E) e assume-se que essas superfícies fazem um ângulo de $45^{\circ}$ com dois eixos coordenados. Logo, o vetor normal nessas superfícies toma a forma: $\mathbf{n}=\left( \pm \frac{\sqrt{2}}{2}, \pm \frac{\sqrt{2}}{2}, 0\right), \mathbf{n}=\left( \pm \frac{\sqrt{2}}{2}, 0, \pm \frac{\sqrt{2}}{2}\right)$ ou $\mathbf{n}=\left(0, \pm \frac{\sqrt{2}}{2}, \pm \frac{\sqrt{2}}{2}\right)$.

Para ilustrarmos a aplicação das condições do contorno na superfície livre nessas superfícies, considere uma célula de superfície que tem as faces $\left(i+\frac{1}{2}\right)$ e $\left(k+\frac{1}{2}\right)$ em contato com faces de células vazias (ver figura 3.9). Neste caso, tomamos o vetor normal como sendo

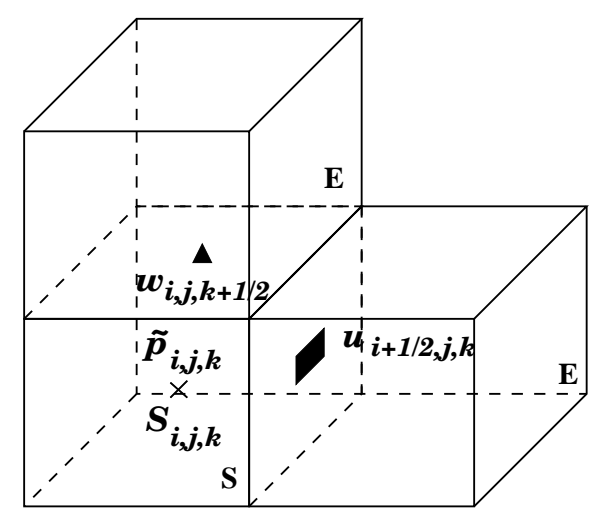

Figura 3.9: Células de superfícies (S) com duas faces em contato com faces de célula vazia (E).

$$
\mathbf{n}=\left(\frac{\sqrt{2}}{2}, 0, \frac{\sqrt{2}}{2}\right) \text { e os vetores tangencias dados por } \mathbf{m}_{1}=\left(\frac{\sqrt{2}}{2}, 0,-\frac{\sqrt{2}}{2}\right) \text { e } \mathbf{m}_{\mathbf{2}}=(0,1,0) \text {. }
$$


Introduzindo esses vetores nas equações (3.16)-(3.17) obtemos

$$
\begin{gathered}
\tilde{p}=\frac{1}{R e}\left(\frac{\partial u}{\partial x}+\frac{\partial w}{\partial z}+\frac{\partial u}{\partial z}+\frac{\partial w}{\partial x}\right)+\frac{1}{2}\left(S^{x x}+S^{y y}\right)+S^{x y}=0, \\
\frac{1}{R e}\left(\frac{\partial u}{\partial x}-\frac{\partial w}{\partial z}\right)+S^{x y}+S^{y z}=0,
\end{gathered}
$$

respectivamente. Com referência a figura 3.9, os valores de $u_{i+\frac{1}{2}, j, k}, w_{i, j, k+\frac{1}{2}}$ nas faces das células vazias e a pressão $\tilde{p}_{i, j, k}$ são requeridas. Esses valores são obtidos discretizando a equação (3.102) e a equação de conservação de massa no centro da célula computacional como segue.

$$
\begin{aligned}
& \frac{1}{R e}\left(\frac{u_{i+\frac{1}{2}, j, k}-u_{i-\frac{1}{2}, j, k}}{\delta x}-\frac{w_{i, j, k+\frac{1}{2}}-w_{i, j, k-\frac{1}{2}}}{\delta z}\right)+S_{i, j, k}^{x y}+S_{i, j, k}^{y z}=0 \\
& \frac{u_{i+\frac{1}{2}, j, k}-u_{i-\frac{1}{2}, j, k}}{\delta x}+\frac{w_{i, j, k+\frac{1}{2}}-w_{i, j, k-\frac{1}{2}}}{\delta z}=-\left(\frac{v_{i, j+\frac{1}{2}, k}-v_{i, j-\frac{1}{2}, k}}{\delta y}\right) .
\end{aligned}
$$

Resolvendo (3.103) e (3.104) para $u_{i+\frac{1}{2}, j, k}$ e $w_{i, j, k+\frac{1}{2}}$ obtemos as seguintes expressões:

$$
\begin{aligned}
u_{i+\frac{1}{2}, j, k} & =u_{i-\frac{1}{2}, j, k}-\frac{1}{2} \frac{\delta x}{\delta y}\left(v_{i, j+\frac{1}{2}, k}-v_{i, j-\frac{1}{2}, k}\right)+\frac{\delta x}{4} \frac{1}{R e}\left(S_{i, j, k}^{x y}-S_{i, j, k}^{y z}\right) \\
w_{i, j, k+\frac{1}{2}} & =w_{i, j, k-\frac{1}{2}}-\frac{1}{2} \frac{\delta z}{\delta y}\left(v_{i, j+\frac{1}{2}, k}-v_{i, j-\frac{1}{2}, k}\right)+\frac{\delta z}{4} \frac{1}{R e}\left(S_{i, j, k}^{x y}-S_{i, j, k}^{y z}\right) .
\end{aligned}
$$

Tendo calculado as velocidades nas faces das células vazias, a pressão no centro da célula de superfície é obtida usando a equação (3.101) aplicada no centro da célula,

$$
\begin{aligned}
\tilde{p}_{i, j, k}= & \frac{1}{R e}\left(\frac{u_{i+\frac{1}{2}, j, k}-u_{i-\frac{1}{2}, j, k}}{\delta x}+\frac{w_{i, j, k+\frac{1}{2}}-w_{i, j, k-\frac{1}{2}}}{\delta z}+\frac{u_{i, j, k+\frac{1}{2}}-u_{i, j, k-\frac{1}{2}}}{\delta z}\right. \\
& \left.\left.+\frac{w_{i+\frac{1}{2}, j, k}-w_{i-\frac{1}{2}, j, k}}{\delta x}\right)+\frac{1}{2}\left(S_{i, j, k}^{x x}+S_{i, j, k}^{y y}\right)+S_{i, j, k}^{x y}\right) .
\end{aligned}
$$

Para outras configurações de superfícies-2D, a aplicação das condições de contorno na superfície livre é feita de maneira análoga ao caso acima.

iii) Superfícies-3D: Essas superfícies são classificadas como células de superfície (S) que possuem três faces adjacentes em contato com faces de células vazias (E). Nessas superfícies, o vetor normal unitário faz um ângulo de $60^{\circ} \mathrm{com}$ os eixos coordenados e pode ser escrito na forma: $\mathbf{n}=\left( \pm \frac{\sqrt{3}}{3}, \pm \frac{\sqrt{3}}{3}, \pm \frac{\sqrt{3}}{3}\right)$. Por exemplo, se considerarmos uma célula de superfície com as faces $\left(i+\frac{1}{2}\right),\left(j+\frac{1}{2}\right)$ e $\left(k+\frac{1}{2}\right)$ em contato com faces de células vazias (ver figura 3.10) tomamos $\mathbf{n}=\left(\frac{\sqrt{3}}{3}, \frac{\sqrt{3}}{3}, \frac{\sqrt{3}}{3}\right), \mathbf{m}_{\mathbf{1}}=\left(\frac{\sqrt{2}}{2},-\frac{\sqrt{2}}{2}, 0\right)$ e $\mathbf{m}_{\mathbf{2}}=\left(\frac{\sqrt{6}}{6}, \frac{\sqrt{6}}{6},-2 \frac{\sqrt{6}}{6}\right)$. Substituindo $\mathbf{n}, \mathbf{m}_{\mathbf{1}}$ e $\mathbf{m}_{\mathbf{2}}$ nas equações (3.16)-(3.17) obtemos 


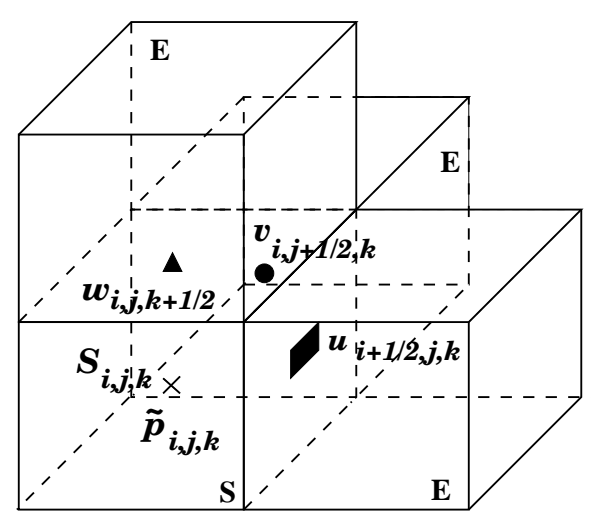

Figura 3.10: Células de superfícies (S) com duas faces em contato com faces de célula vazia (E).

$$
\begin{gathered}
\tilde{p}=\frac{2}{3 R e}\left(\frac{\partial u}{\partial y}+\frac{\partial v}{\partial x}+\frac{\partial u}{\partial z}+\frac{\partial w}{\partial x}+\frac{\partial v}{\partial z}+\frac{\partial w}{\partial y}\right)+\frac{1}{3}\left(S^{x x}+S^{y y}+S^{z z}\right)+\frac{2}{3}\left(S^{x y}+S^{x z}+S^{y z}\right) \\
\frac{1}{R e}\left[2\left(\frac{\partial u}{\partial x}+\frac{\partial v}{\partial y}\right)+\left(\frac{\partial u}{\partial z}+\frac{\partial w}{\partial x}\right)-\left(\frac{\partial v}{\partial z}+\frac{\partial w}{\partial y}\right)\right]+S^{x x}-S^{y y}+S^{x z}-S^{y z}=0, \\
\frac{1}{R e}\left[-6 \frac{\partial w}{\partial z}+2\left(\frac{\partial u}{\partial y}+\frac{\partial v}{\partial x}\right)-\left(\frac{\partial u}{\partial z}+\frac{\partial w}{\partial x}\right)-\left(\frac{\partial v}{\partial z}+\frac{\partial w}{\partial y}\right)\right]+S^{x x}+S^{y y}-2 S^{z z}-S^{x z}-S^{y z}=0
\end{gathered}
$$

respectivamente. Neste caso, os valores de $u_{i+\frac{1}{2}, j, k}, v_{i, j+\frac{1}{2}, k}$ e $w_{i, j, k+\frac{1}{2}}$ são requeridos. Esses valores são obtidos discretizando as equações (3.109), (3.110) e a equação da conservação de massa no centro da célula computacional obtendo assim um sistema linear $3 \times 3$ para as incógnitas $u_{i+\frac{1}{2}, j, k}, v_{i, j+\frac{1}{2}, k}$ e $w_{i, j, k+\frac{1}{2}}$. Esse sistema linear é resolvido pelo método de eliminação de Gauss. Depois de calculadas as velocidades nas faces das células de superfícies calculs-se a pressão discretizando a equação (3.108) no centro da célula. Detalhes dos cálculos podem ser encontrados em Tomé et al. [82]. A outras configurações de superfícies-3D são tratadas de maneira análoga.

Detalhes sobre o tratamento de superfícies-1D, 2D e 3D podem ser encontrados em Tomé et al. [82].

\subsubsection{Cálculo do Passo no tempo}

Em cada ciclo de cálculo, o método GENSMAC3D [80] determina o tamanho do passo no tempo $\delta t$ que é calculado segundo as seguintes restrições de estabilidade:

$$
\delta t<\frac{\delta x}{|u|}, \quad \delta t<\frac{\delta y}{|v|}, \quad \delta t<\frac{\delta z}{|w|}
$$

Esta restrição garante que nenhuma partícula pode cruzar mais do que uma célula em um dado intervalo de tempo.

Devido à discretização explícita da equação de quantidade de movimento, adota-se a seguinte 
restrição de estabilidade que envolve o número de Reynolds e a viscosidade:

$$
\delta t<\frac{\delta x^{2} \delta y^{2} \delta z^{2}}{\delta x^{2} \delta y^{2}+\delta x^{2} \delta z^{2}+\delta y^{2} \delta z^{2}} \frac{R e}{2}
$$

A implementação dessas restrições para o tamanho do passo no tempo seguem as mesma idéias de Tomé \& McKee. [85]. Detalhes sobre a implementação do cálculo do passo no tempo pode ser encontrado em Tomé et al. [80].

\subsection{Simulação numérica de escoamentos viscoelásticos tridimensionais com superfícies livres}

As equações de diferenças finitas desenvolvidas nas seções anteriores foram incorporadas no ambiente de simulação Freeflow3D aumentando assim a aplicabilidade desse ambiente para escoamentos viscoelásticos governados pela equação constitutiva PTT. No capítulo 3, foram considerados o problema do jato oscilante e o problema do inchamento do extrudado, os quais foram estudados utilizando o código Freeflow2D. Nessa seção, vamos mostrar que Freeflow3D pode simular escoamentos tridimensionais com superfícies livres modelados pela equação PTT e aplica-lo ao problema do jato oscilante e inchamento do extrudado.

\subsubsection{Simulação numérica do jato oscilante}

No Capítulo 2, Seção 2.9.1 vimos que no momento em que um jato incide em uma superfície rígida plana, se o número de Reynolds for suficientemente pequeno então pode ocorrer o fênomeno conhecido como flambagem ou jato oscilante. Também foi mostrado que se a viscoelasticidade, representada pelo número de Weissenberg, for grande o suficiente, então o jato apresentará o fenômeno do jato oscilante.

Cruickshank \& Munson [23] realizaram uma série de experimentos com jatos Newtonianos e obtiveram condições para a ocorrência do jato oscilante. Essas condições envolvem o número de Reynolds, o diâmetro do jato e a altura do injetor até a placa rígida. Em particular, Cruickshank \& Munson [23] mostraram experimentalmente que um jato Newtoniano axissimétrico apresenta o fenômeno do jato oscilante se as seguintes condições forem satisfeitas:
a) $\operatorname{Re}<1.2$,
b) $H / D>7.2$.

Para demonstrar esse fato, realizamos duas simulações de um jato incindindo em uma placa rígida em que as condições (3.113) são satisfeitas (Problema I). Em uma simulação empregamos um jato Newtoniano e a outra utilizamos um jato modelado pela equação constitutiva PTT. O domínio computacional adotado nessas simulações é apresentado na figura 3.11 e os dados geométricos utilizados estão dispostos na Tabela 3.1.

Da Tabela 3.1 vemos que $H / D=20.0>7.2$ de modo que a condição 3.113 b está satisfeita. Para que a condição 3.113 a seja satisfeita, escolhemos $R e=0.5$ para ambos os jatos. Os 
Figura 3.11: Domínio computacional: $7 \mathrm{~cm} \times 7 \mathrm{~cm} \times 12.8 \mathrm{~cm} ; 70 \times 70 \times 128$ células. Placa rígida de $7 \mathrm{~cm} \times 7 \mathrm{~cm} \times 3 \mathrm{~mm}$. Injetor de $6 \mathrm{~mm}$ de diâmetro. Altura do injetor até a placa rígida de $12 \mathrm{~cm}$.

\section{Dados geométricos}

\begin{tabular}{|l|l|}
\hline domínio & $7 \mathrm{~cm} \times 7 \mathrm{~cm} \times 12.8 \mathrm{~cm}$ \\
\hline malha & $70 \times 70 \times 128$ células \\
\hline$H:$ altura do injetor até a placa rígida & $12 \mathrm{~cm}$ \\
\hline$D:$ diâmetro do injetor & $6 \mathrm{~mm}$ \\
\hline
\end{tabular}

Tabela 3.1: Modelagem do problema. Geometria para o Problema I e Problema II .

dados utilizados nessas simulações estão dispostos na Tabela 3.2 na coluna correspondente ao Problema I. Com esses dados o código FreeFlow3d simulou esse problema até o tempo $t=0.575 \mathrm{~s}$. Os resultados obtidos das simulação do jato Newtoniano e da simulação do jato com o fluido PTT onde $R e=0.5$, são apresentados na figura 3.12. Como podemos observar na figura 3.12, o jato Newtoniano após atingir a placa rígida $(t=0.175 \mathrm{~s})$ sofre um aumento do diâmetro devido a ação da condição não-escorregamento enquanto o jato modelado pela equação PTT parece não apresentar esse efeito. No tempo $t=0.35 \mathrm{~s}$ ambos os jatos ja apresentam o fenômeno do jato oscilante e podemos observar com maior clareza o aumento do diâmetro do jato Newtoniano. Esse efeito foi observado nos experimentos de Cruickshank \& Munson [23] e também por Unilever Research [87]. O jato com o fluido PTT não apresenta esse efeito e o fenômeno do jato oscilante é mais acentuado. Nos tempos subsequentes, vemos que o jato com o fluido PTT apresenta um comportamento bem diferente daquele apresentado pelo fluido Newtoniano. Acreditamos que o comportamento do jato PTT seja devido a viscoelasticidade do fluido.

Para demonstrar que o efeito da viscoelasticidade pode fazer com que um jato tridimensional oscile, realizamos duas outras simulações utilizando um jato Newtoniano e um jato com 
fluido PTT, em que a condição 3.113a não é satisfeita por ambos os jatos. Nessas simulações utilizamos a mesma geometria apresentada na Tabela 3.1 e os restantes dos dados são apresentados na Tabela 3.2 embaixo da coluna Problema II. Nessas simulações utilizamos o número de Reynolds $R e=1.3>1.2$ e portanto a condição 3.113a não é satisfeita. Com esses dados, o código FreeFlow3D simulou esse problema para ambos os jatos até o tempo $t=1.15 \mathrm{~s}$.

Como era esperado pela teoria de Cruickshank, o jato Newtoniano não sofre nenhuma oscilação ao atingir a placa rígida; o número de Reynolds é grande o suficiente para que a inércia domine o efeito viscoso o que faz com que o jato escoe continuamente na direção radial. Porém, o jato com o fluido PTT ao atingir a placa rígida sofre o efeito da condição não-escorregamento e o aumento da viscosidade no jato faz com o número de Reynolds local seja pequeno, fato esse que desacelera o fluido, fazendo com que o jato apresente o efeito do jato oscilante. Essas conclusões são baseadas nos resultados obtidos no capítulo 3 para jatos bidimensionais. Para jatos tridimensionais é necessário ainda fazer um estudo numérico mais aprofundado e obter resultados experimentais para validar os resultados numéricos. Os resultados experimentais estão sendo elaborados pelo Departamento de Engenharia de Polímeros da Universidade do Minho - Guimarães - Portugal e durante a elaboração desse trabalho não obtivemos acesso a esses resultados.

Dados que caracterizam os escoamentos dos fluidos Newtoniano e PTT
\begin{tabular}{|l|l|l|}
\hline Newtoniano e PTT & Problema I Re=0.5 & Problema II Re=1.3 \\
\hline \hline$U:$ velocidade de injeção & $\mathbf{1 . 0} \mathrm{ms}^{-1}$ & $\mathbf{0 . 5} \mathrm{ms}^{-1}$ \\
\hline$g_{z}:$ força gravitacional & $-9.81 \mathrm{~ms}^{-2}$ & $-9.81 \mathrm{~ms}^{-2}$ \\
\hline$\eta$ & $12 \mathrm{Pa.s}$ & $2.3076 \mathrm{~Pa} . \mathrm{s}$ \\
\hline$\rho$ & $1000 \mathrm{kgm}^{-3}$ & $1000 \mathrm{kgm}^{-3}$ \\
\hline$R e$ & $\mathbf{0 . 5}$ & $\mathbf{1 . 3}$ \\
\hline$F r$ & 0.242611 & 0.485222 \\
\hline Definição do modelo PTT & & \\
\hline$\lambda$ & $\mathbf{0 . 0 0 6} \mathrm{s}$ & $\mathbf{0 . 0 1 2} \mathrm{s}$ \\
\hline$\varepsilon$ & 0.01 & 0.01 \\
\hline$\xi$ & 0.01 & 0.01 \\
\hline$W e$ & 1.0 & 1.0 \\
\hline
\end{tabular}

Tabela 3.2: Simulação numérica do jato oscilante: Dados do fluido Newtoniano e viscoelásticos. 

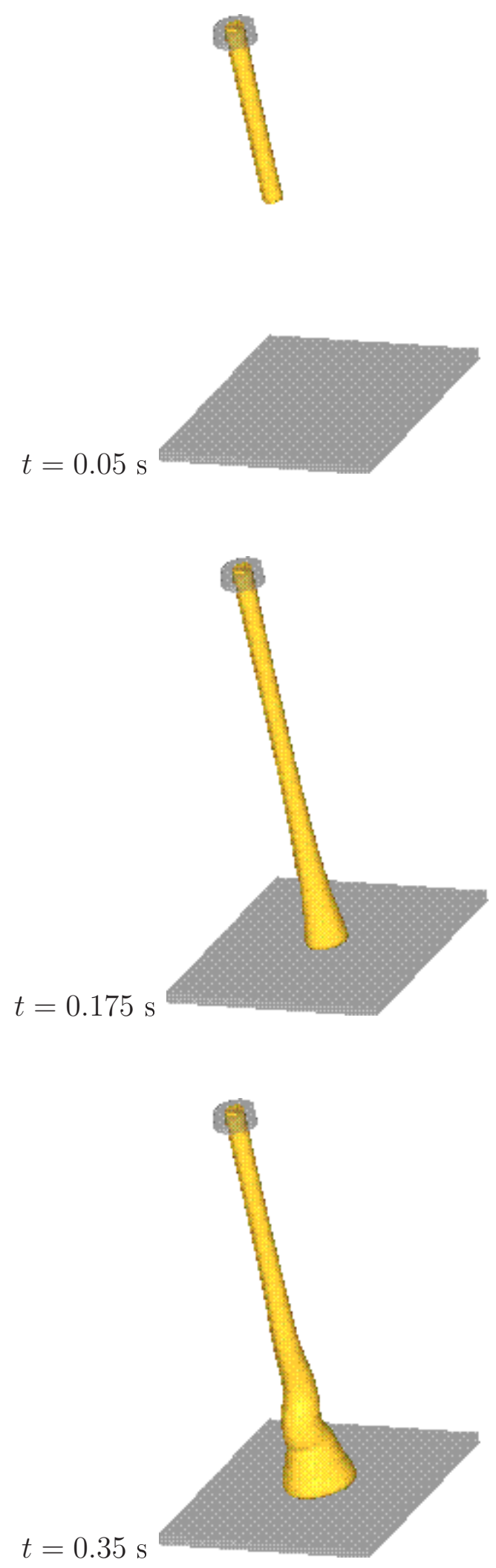
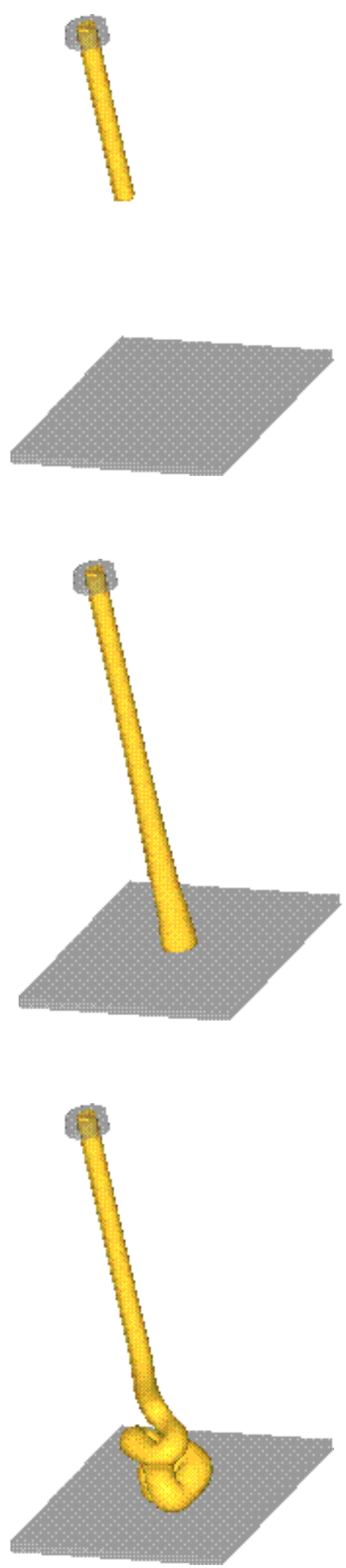

Figura 3.12: Simulação numérica do jato oscilante. Configuração do fluido em diferentes tempos. Coluna a esquerda - jato Newtoniano; coluna a direita - jato PTT. $R e=0.5, W e=1$, $\xi=0.01$ e $\varepsilon=0.01$. 

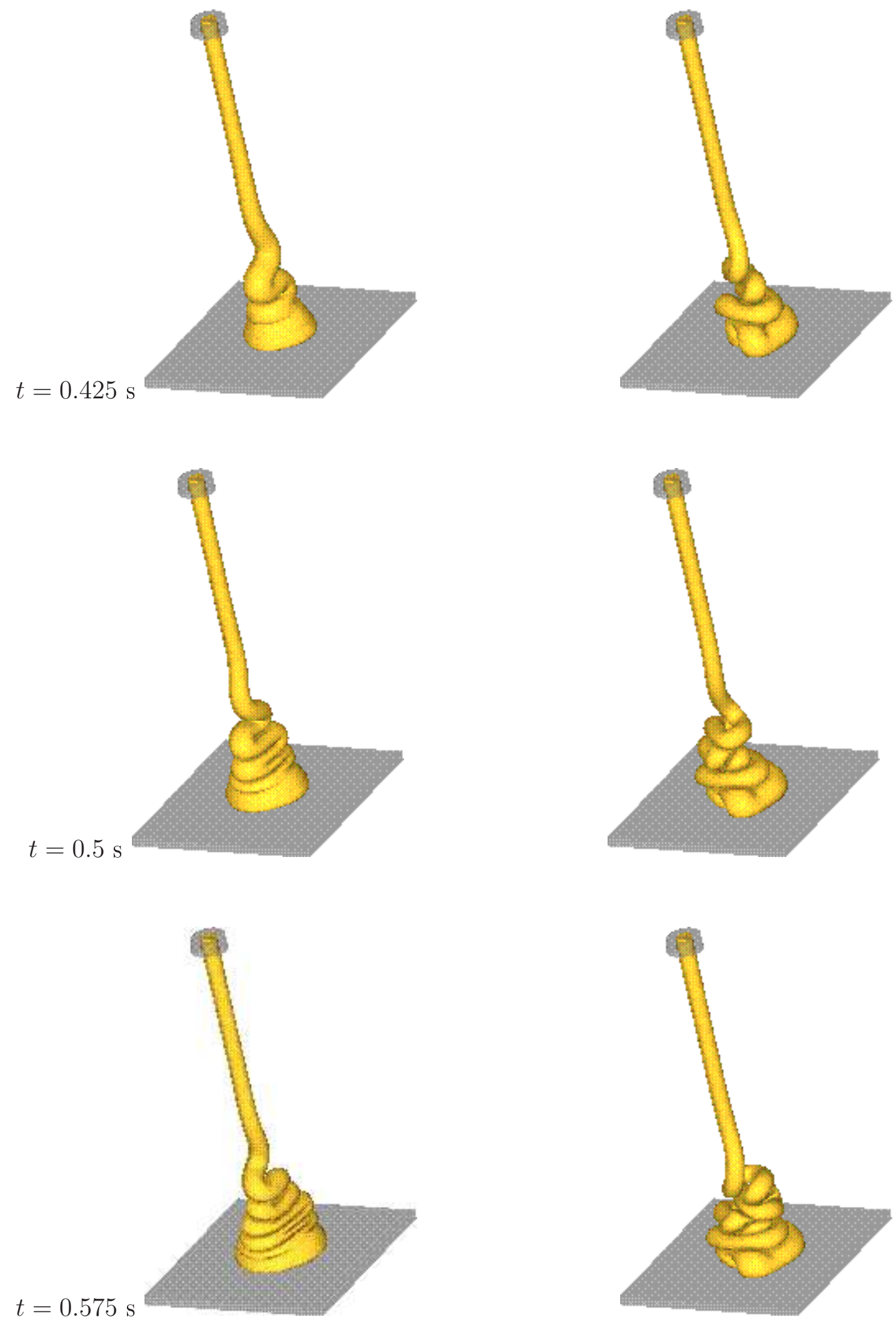

Figura 3.12. Continuação. Configuração do fluido em diferentes tempos. Coluna a esquerda jato Newtoniano e coluna a direita - jato PTT. $R e=0.5, W e=1, \xi=0.01$ e $\varepsilon=0.01$. 

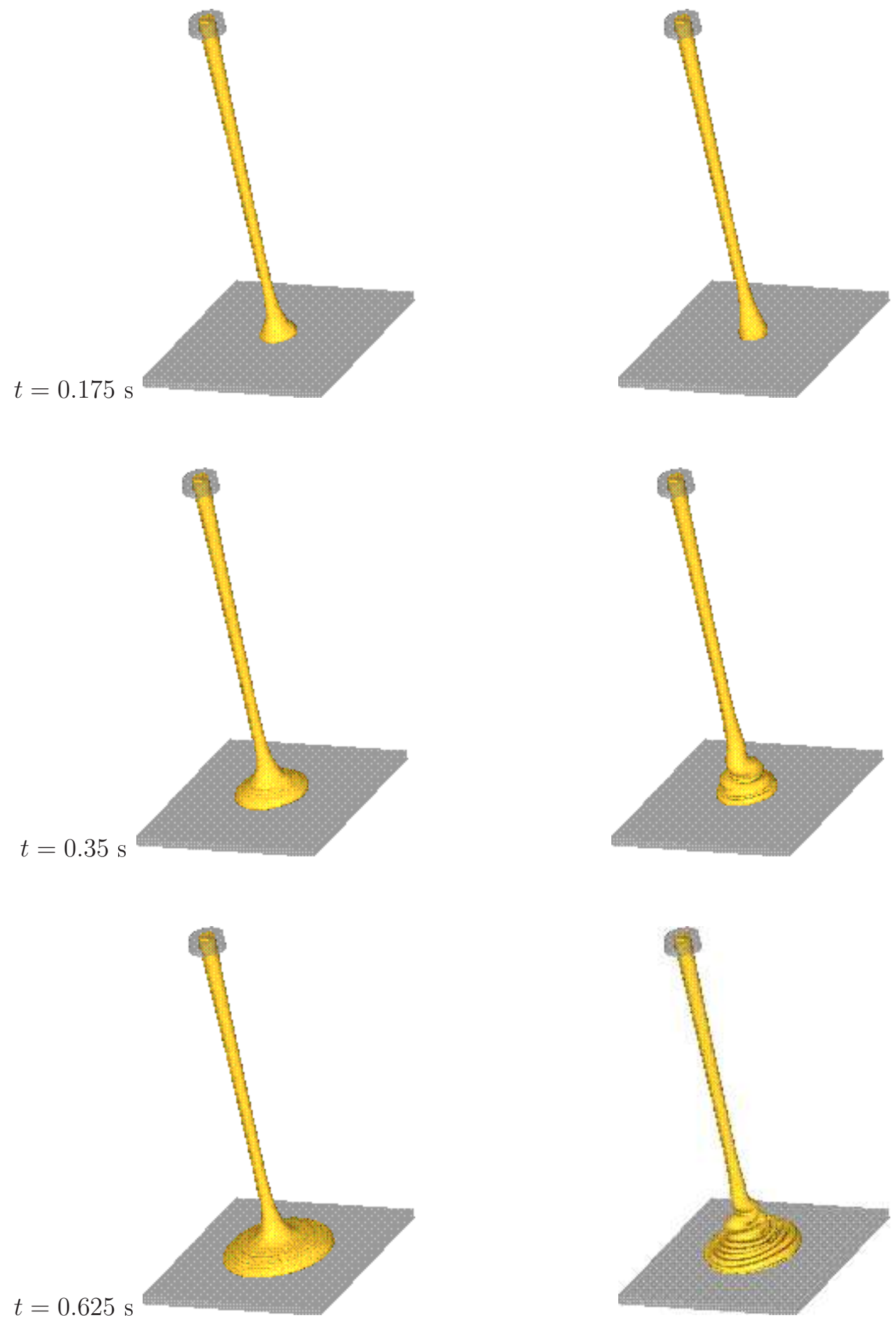

Figura 3.13: Simulação numérica do jato oscilante. Configuração do fluido em diferentes tempos. Coluna a esquerda - jato Newtoniano; coluna a direita - jato PTT. $R e=1.3, W e=1$, $\xi=0.01$ e $\varepsilon=0.01$. 

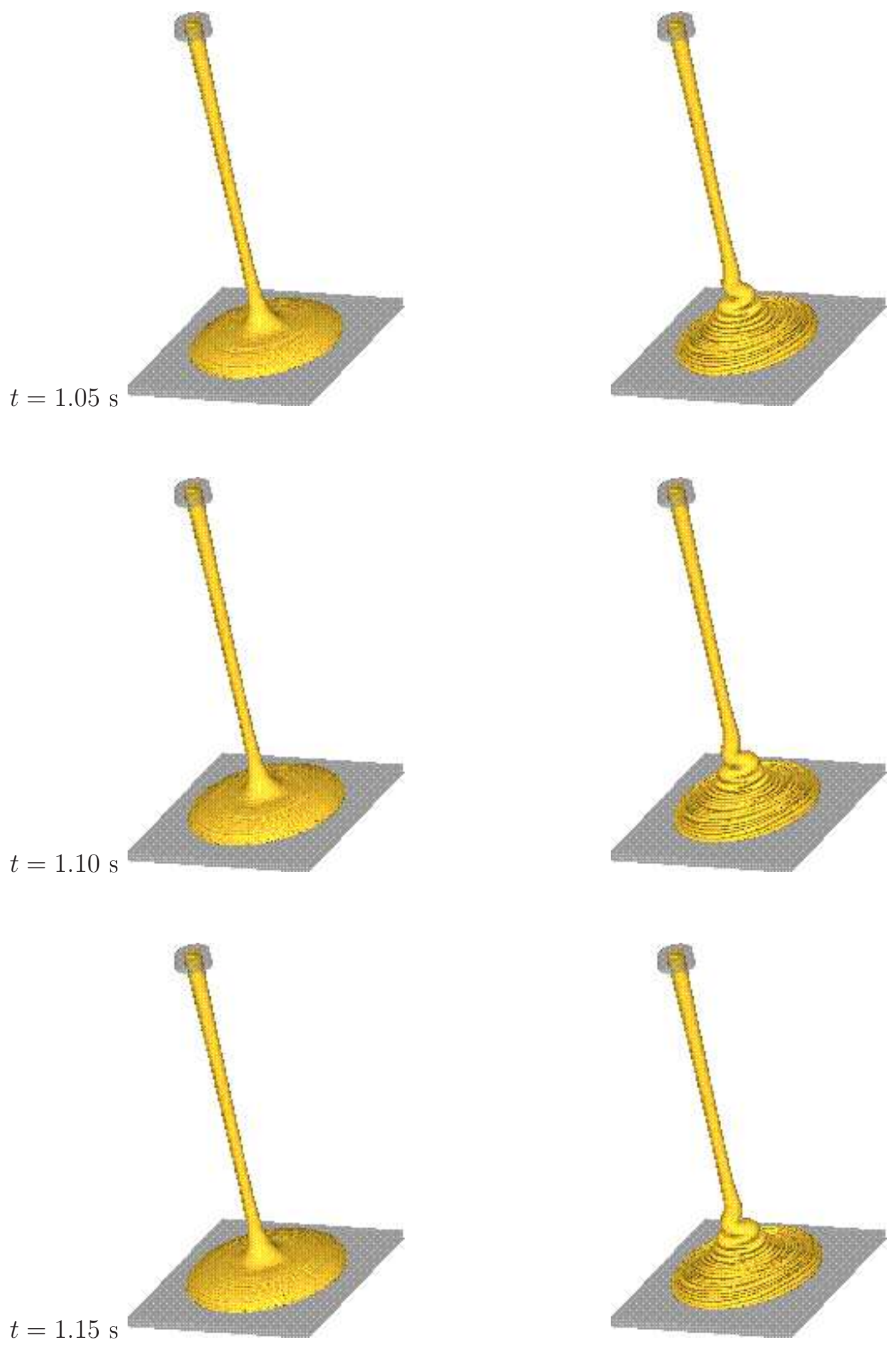

Figura 3.13. Continuação. Configuração do fluido em diferentes tempos. Coluna a esquerda jato Newtoniano; coluna a direita - jato PTT. $R e=1.3, W e=1, \xi=0.01$ e $\varepsilon=0.01$. 


\subsubsection{Simulação numérica do inchamento do extrudado}

No momento em que um jato é expelido na atmosfera pode ocorrer um fênomeno conhecido como inchamento do extrudado, que é caracterizado pelo aumento do diâmetro do extrudado em relação ao diâmetro interno do tubo pelo qual o jato é expelido (ver figura 3.14). O inchamento do extrudado é calculado pela relação entre a dilatação sofrida pelo jato após emergir a atmosfera $\left(D_{\max }\right)$ e o diâmetro do tubo $(D)$ que o fluido ocupava (ver figura 3.14). Assim, define-se a taxa de inchamento $S_{r}$ como sendo $S_{r}=\frac{D_{\max }}{D}$. Esse fenômeno aparece em muitas aplicações industriais e tem atraído a atenção de vários pesquisadores e várias técnicas para simular o inchamento do extrudado tem sido propostas, por exemplo [77], [68], [21], [38], [39] e [84].

Para simular esse problema, vamos considerar o escoamento de um fluido viscoelástico dentro de um tubo circular onde ao final do tubo o fluido é expelido para a atmosfera. Os dados geométricos do problema estão dispostos na Tabela 3.3 e o domínio computacional é ilustrado na figura 3.14. Na entrada do tubo, a velocidade foi definida utilizando a solução analítica apresentada por Alves et al. [2] dada por:

$$
\begin{aligned}
W(x, y)= & \frac{R e \frac{d p}{d z}}{2 \chi}\left(1-r^{2}\right)+\frac{1}{R e W e^{2} \xi(2-\xi) \frac{d p}{d z}}\left(1+\frac{2}{\chi}\right)\left\{\ln \left[\frac{1+\sqrt{1-(a r)^{2}}}{1+\sqrt{1-a^{2}}}\right]\right. \\
& \left.+\sqrt{1-a^{2}}-\sqrt{1-(a r)^{2}}\right\}
\end{aligned}
$$

onde

$$
r=\sqrt{x^{2}+y^{2}}, \quad a=-2 \operatorname{Re} W e \frac{d p}{d z} \sqrt{\xi(2-\xi)}, \quad \chi=\frac{\xi(2-\xi)}{\varepsilon(1-\xi)}
$$

e na parede do tubo foi imposta a condição não-escorregamento.

Os valores das componentes do tensor não-Newtoniano $S_{i j}$ na parede do tubo formam calculadas pelas expressões derivadas na Seção 3.4 enquanto que na entrada do tubo as componentes do tensor não-Newtoniano $S_{i j}$ foram calculadas por (ver Tomé et al. [82])

$$
\begin{aligned}
& S^{x x}=0, \quad S^{y y}=0, \quad S^{x y}=0, \\
& S^{x z}=-\frac{1}{R e} \frac{\partial w}{\partial x}, \quad S^{y z}=\frac{1}{R e} \frac{\partial w}{\partial y}, \quad S^{z z}=2 \frac{W e}{R e}\left[\left(\frac{\partial w}{\partial x}\right)^{2}+\left(\frac{\partial w}{\partial y}\right)^{2}\right]
\end{aligned}
$$

Foram realizadas várias simulações onde fixamos o número de Reynolds $R e=1$ e foram adotados diferentes números de Weissenberg. Os dados que caracterizam os escoamentos dos fluidos viscoelásticos estão dispostos na tabela 3.4. Os parâmetros do modelo PTT foram mantidos fixos em todas as simulações, $\varepsilon=0.15, \xi=0.05$ e $\Delta \mathrm{p}_{z}=\partial p / \partial z=-0.25$. 


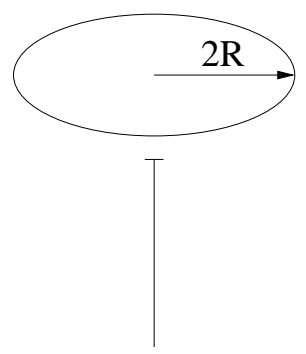

$10 \mathrm{~m}$

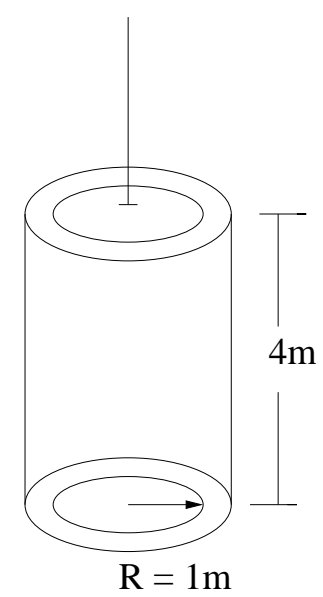

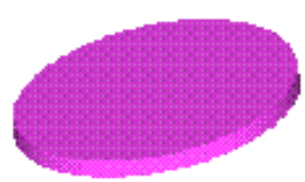

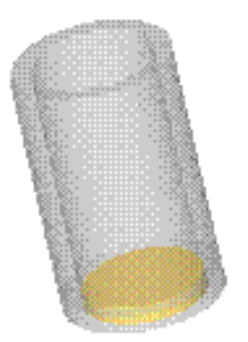

Figura 3.14: Domínio geométrico: $4.6 \times 4.6 \times 14.6 \mathrm{~m}$; Raio do injetor: $1 \mathrm{~m}$; compimento do tubo: $4 \mathrm{~m}$; Distância da extremidade do tudo até o ejetor: $10 \mathrm{~m}$. Malha utilizada: $46 \times 46 \times 146$ células $(\delta x=\delta y=\delta z=0.1 \mathrm{~m})$.

Dados geométricos

\begin{tabular}{|l|l|}
\hline domínio & $4.6 \mathrm{~m} \times 4.6 \mathrm{~m} \times 14.6 \mathrm{~m}$ \\
\hline malha & $46 \times 46 \times 146$ células \\
\hline diâmetro do injetor $D=2 R(R$ - raio $)$ & $2 \mathrm{~m}$ \\
\hline diâmetro do ejetor & $4.6 \mathrm{~m}$ \\
\hline comprimento do tudo & $4 \mathrm{~m}$ \\
\hline distância do final do tubo ao ejetor: $5 D=10 R$ & $10 \mathrm{~m}$ \\
\hline
\end{tabular}

Tabela 3.3: Modelagem do problema.

A figura 3.16 mostra a visualização tridimensional dos resultados obtidos nas simulações do inchamento do extrudado para $R e=1$ e $W e=0.1,1,3$ nos tempos $t=8 \mathrm{~s}, t=15 \mathrm{~s}, t=30 \mathrm{~s}$. A visualização frontal desses resultados é mostradas na figura 3.17.

Na figura 3.16 podemos observar que no tempo $t=8 \mathrm{~s}$ os jatos mantêm-se dentro do tubo, não sendo possível observar grandes diferenças oriundas da variação do número de Weissenberg; apenas o fato de $W_{\max }$ variar o que causa o jato correspondente a $W e=3$ estar um pouco mais avançado com relação aos jatos com $W e=0.1$ e $W e=1$ (ver figura 3.17). Porém, no 


\begin{tabular}{|c|c|c|c|c|c|}
\hline$W e$ & 0.1 & 0.5 & 1 & 2 & 3 \\
\hline \hline$W_{\text {max }}\left[\mathrm{ms}^{-1}\right]$ & 1.02647 & 1.02719 & 1.02945 & 1.0388 & 1.05553 \\
\hline$\eta[\mathrm{Pa} . \mathrm{s}]$ & 1026.47 & 1027.19 & 1029.45 & 1038.80 & 1055.53 \\
\hline$\rho\left[\mathrm{kgm}^{-3}\right]$ & 1000 & 1000 & 1000 & 1000 & 1000 \\
\hline$F r$ & 0.3277 & 0.3279 & 0.3286 & 0.3316 & 0.3370 \\
\hline$\lambda[\mathrm{s}]$ & 0.0974 & 0.4867 & 0.9713 & 1.9252 & 2.8421 \\
\hline
\end{tabular}

Tabela 3.4: Dados utilizados na simulação do inchamento do extrudado para $R e=1$.

tempo $t=15$ s podemos notar uma expressiva variação no inchamento extrudado a medida que o número de Weissenberg aumenta e no tempo $t=30$ s podemos observar que o jato correspondente ao número de Weissenberg $W e=3$ apresenta o maior inchamento. O gráfico mostrado na figura 3.15 mostra a variação do inchamento em função do número de Weissenberg $W$ e. O inchamento foi medido a partir de um corte no plano YZ passando pelo centro do jato. O gráfico mostra que a razão de inchamento $S_{r}$ obtida foi $8.1 \%$ para $W e=0.1,18.8 \%$ para $W e=1$ e $29.20 \%$ para $W e=3$. Esses resultados mostram que o método numérico apresentado nesse capítulo é capaz de simular escoamentos com alta viscoelasticidade.

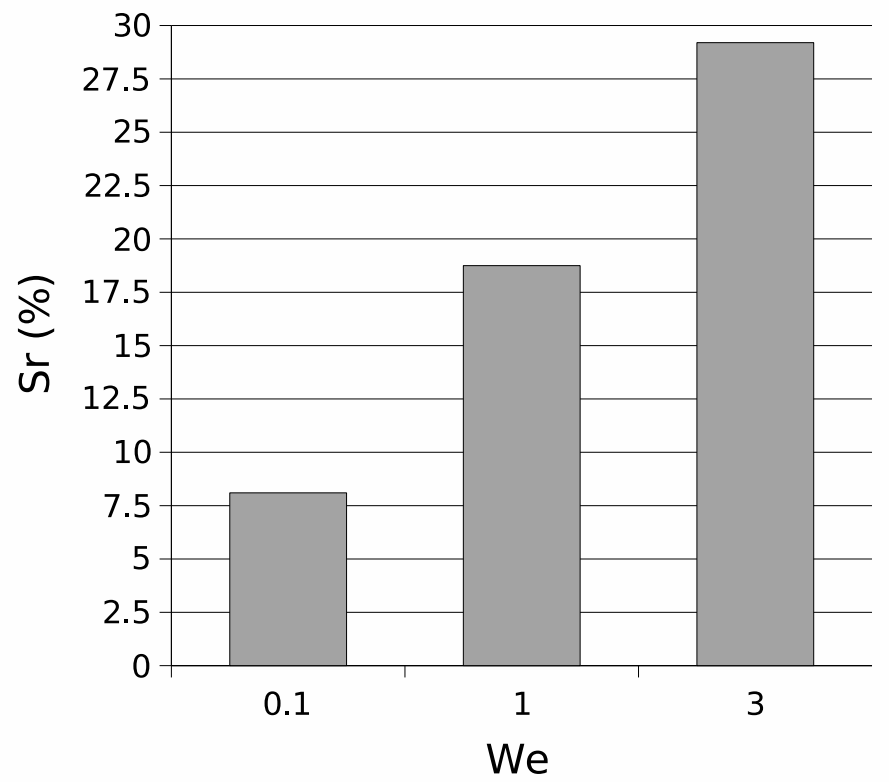

Figura 3.15: Razão de inchamento obtida no tempo $t=30 \mathrm{~s}$. $R e=1$ e $W e=0.1,1$ e 3 . 

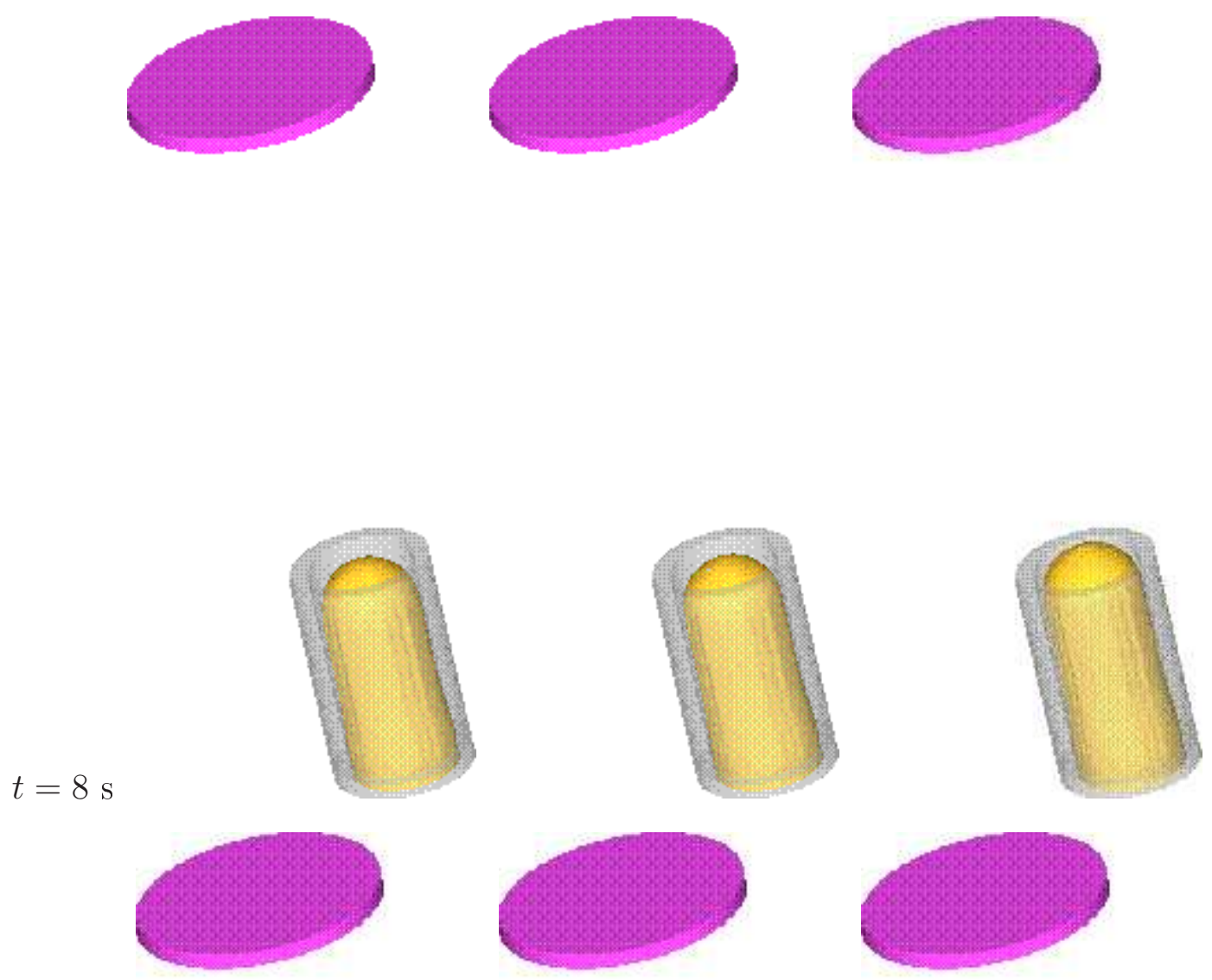

$$
t=15 \mathrm{~s}
$$
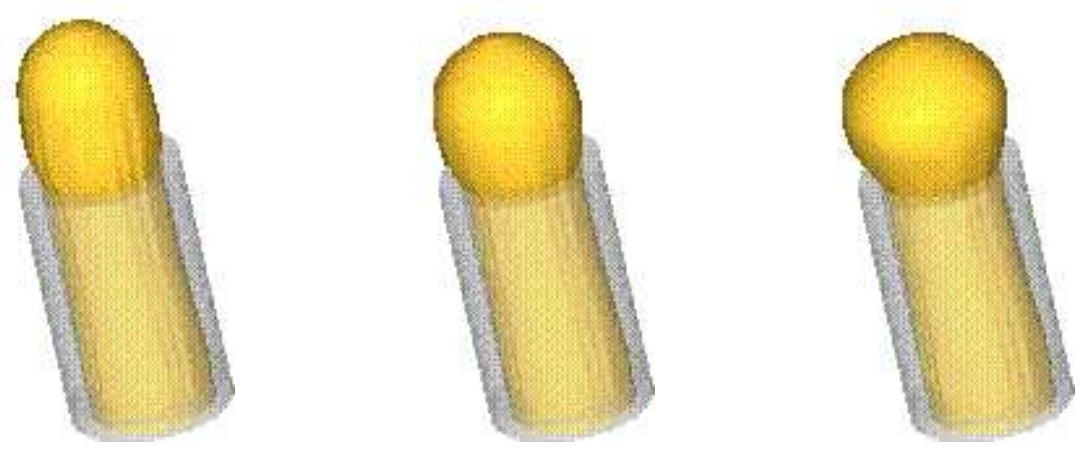

Figura 3.16: Simulação do inchamento do extrudado. Visualização tridimensional do inchamento do extrudado em diferentes tempos. $R e=1$ e $W e=0.1,1,3$. 
$t=30 \mathrm{~s}$
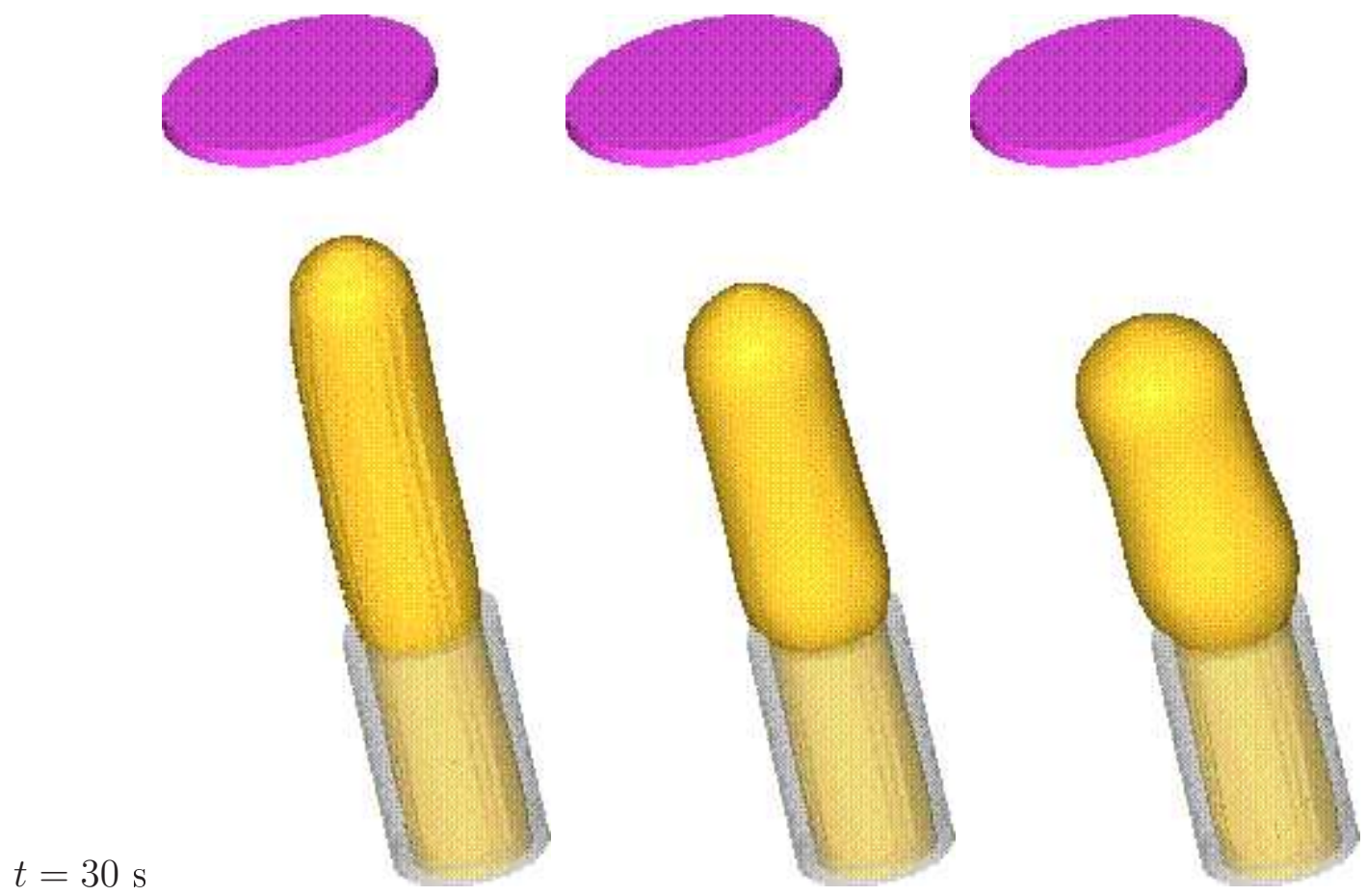

Figura 3.16. Continuação. Visualização do fluido em diferentes tempos. Re $=1 \mathrm{e}$ $W e=0.1,1,3$.

$$
t=8 \mathrm{~s}
$$
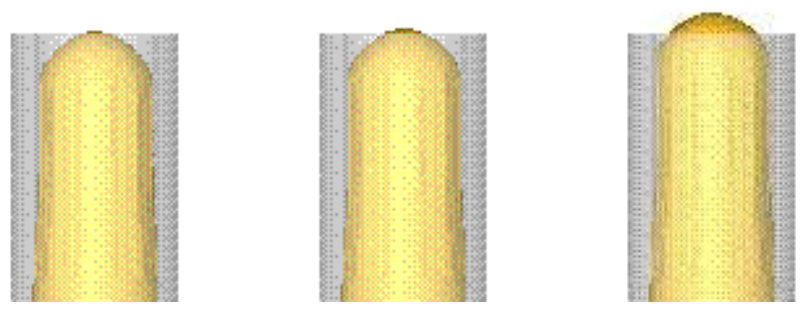

Figura 3.17: Simulação do inchamento do extrudado. Visualização pela face direita do inchamento do extrudado em diferentes tempos. $R e=1$ e $W e=0.1,1,3$. 

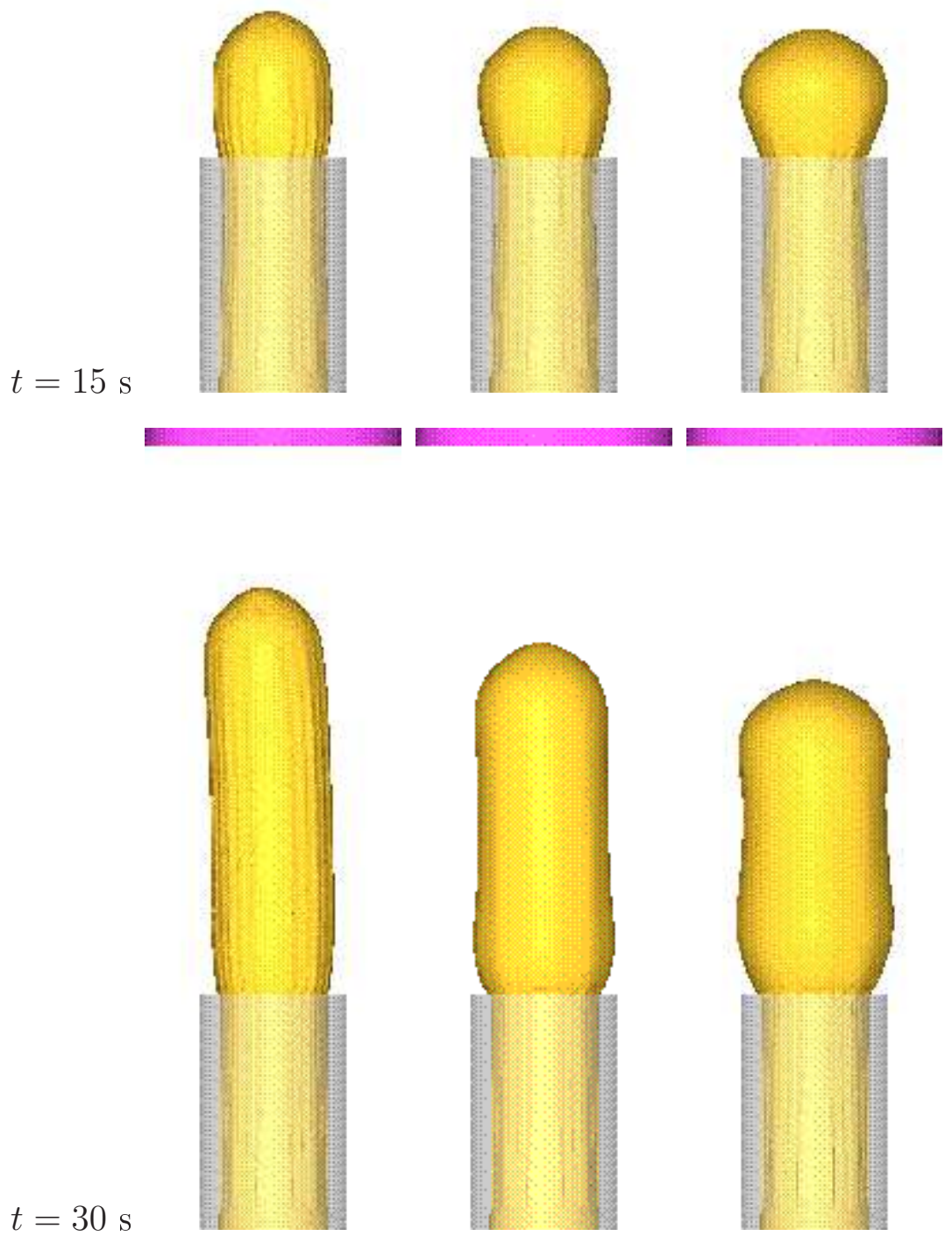

Figura 3.17. Continuação. Visualização pelo face direita do inchamento do extrudado em diferentes tempos. $R e=1$ e $W e=0.1,1,3$. 


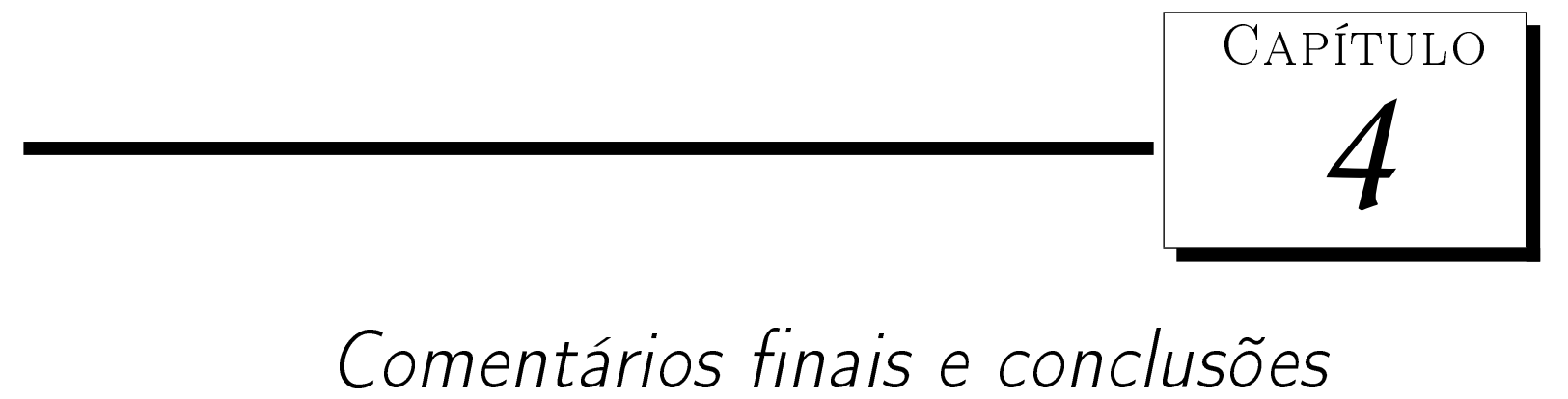

Esta tese apresentou os desenvolvimentos realizados para a obtenção de métodos numéricos para simular escoamentos incompressíveis com superfícies livres governados pela equação constitutiva PTT (Phan-Thien-Tanner). Esse trabalho constituiu-se de duas partes principais: escoamentos bidimensionais e escoamentos tridimensionais. Para a primeira parte foram desenvolvidos dois métodos numéricos conforme apresentados nos Capítulos 1 e 2 dessa tese. Esse trabalho, podemos classificar como uma dificuldade inicial da pesquisa que nos trouxe muito aprendizado na modelagem matemática dos modelos viscoelásticos. Para obter um método estável e consistente usamos a formulação EVSS (Elastic-Viscous Stress-Splitting) [64] que consiste em separar o tensor $\tau_{i j}$ em duas componentes, uma componente chamada de contribuição Newtoniana do fluido e a outra conhecida como contribuição não-Newtoniana. Basicamente, a diferença entre os dois métodos numéricos bidimensionais está na maneira de como foi aplicada a formulação EVSS. Para o primeiro método bidimensional definiu-se um parâmetro $\beta$ o qual relaciona a viscosidade da contribuição Newtoniana $\eta_{N}$ e a viscosidade polimérica $\eta_{P}$. Em seguida aplicou-se a formulação EVSS. Nesta fase, inicialmente seguimos as idéias apresentadas por Xue et al. [92] onde a formulação EVSS é aplicada duas vezes: uma primeira vez onde o tensor extra-tensão, $\tau_{i j}$, é escrito como uma soma de um tensor Newtoniano $D_{i j}$ e um tensor não-Newtoniano $S_{i j}$ e essa transformação é utilizada para se obter um termo difusivo na equação de conservação de quantidade de movimento; uma segunda vez, em que o tensor não-Newtoniano $S_{i j}$ é escrito novamente como como uma soma de um tensor não-Newtoniano $\Sigma_{i j}$ e um tensor Newtoniano, o qual é substituido na equação constitutiva para se obter uma equação para o tensor $\Sigma_{i j}$. Xue et al. [92] utilizaram um método de volume finitos baseado no algoritmo SIMPLEST [91] e apresentaram resultados para escoamentos tridimensionais em uma contração planar. No nosso caso, o emprego dessas duas transformações não resultou em bons resultados e resolvemos então empregar somente a primeira transformação e introduzi-la simul- 
taneamente na equação de conservação de quantidade de movimento e na equação constitutiva obtendo assim o termo difusivo e também uma equação para o tensor não-Newtoniano $S_{i j}$. Esse método numérico está descrito no Capítulo 2, foi implementado no sistema FreeFlow2D e obtivemos resultados numéricos da simulação do jato oscilante e também do impacto de uma gota contra uma superfície rígida. Ainda no Capítulo 1, seguindo as idéias de Alves et al. [2] obtivemos a solução analítica do escoamento totalmente desenvolvido em um canal bidimensional. Assim, para validar o tratamento das componentes do tensor extra-tensão nos contornos rígidos e no interior do domínio computacional simulamos o escoamento de um fluido PTT em um canal bidimensional até atingir o estado estacionário e comparamos os resultados numéricos obtidos com a solução analítica deste problema (ver equações (1.37), (1.52), (1.54) e (1.55), do Capítulo 1. Os resultados mostraram uma boa concordância entre as solução numérica e a solução analítica e o erro relativo entre essas soluções, calculados em três diferentes malhas, demonstrou a convergência do método numérico desenvolvido no Capítulo 1).

No entanto, a formulação adotada no Capítulo 1 introduz um parâmetro adicional $\eta_{N}$ que não está relacionado com a equação constitutiva PTT. Dessa maneira, refizemos a formulação EVSS utilizando somente a viscosidade polimérica $\eta_{P}$, reescrevemos novamente as equações governantes e refizemos o método numérico. Dessa maneira, obtivemos um novo método numérico, que denominamos GENSMAC2D-PTT, que também foi implementado no sistema FreeFlow2D. O método GENSMAC2D-PTT foi desenvolvido especificamente para o tratamento de escoamentos incompressíveis com superfícies livres modelados pela equação constitutiva PTT. O Capítulo 2 apresenta uma descrição detalhada dos items principais de GENSMAC2D-PTT tais como: cálculo do tensor não-Newtoniano nos contornos rígidos, aplicação das condições de contorno na superfície livre, descrição do algoritmo computacional, aplicação do método de diferenças finitas. Alves et. al [2] mostraram que a solução exata para o modelo PTT para um escoamento no estado estacionário é dada pelas equações (2.67)-(2.70). Assim, a validação de GENSMAC2D-PTT seguiu-se de maneira análoga ao primeiro caso e os resultados mostraram boa concordância com a solução analítica e também foi mostrado a convergência de GENSMAC2D-PTT. A solução analítica apresentada por Alves et. al [2] para a componente $\tau^{x x}$ do tensor extra-tensão não depende do parâmetro $\varepsilon$ presente no modelo PTT (ver equação (2.69)). Portanto, uma validação adicional para GENSMAC2D-PTT foi realizada fazendo duas simulações com diferentes valores da constante $\varepsilon$ e comparamos estas soluções numéricas com a expressão analítica de $\tau^{x x}$ e novamente obtivemos uma boa concordância entre as soluções numéricas com $\varepsilon=0.1, \varepsilon=0.25$ e a solução analítica. No Capítulo 2 foram apresentados os resultados numéricos da simulação de escoamentos viscoelásticos obtidos por GENSMAC2D-PTT. Em particular, foram apresentados a simulação do jato oscilante, inchamento do extrudado e o impacto de uma gota em uma superfície rígida. O primeiro exemplo de escoamentos viscoelásticos bidimensionais com superfícies livres apresentado neste trabalho, para ambos os métodos numéricos bidimensionais desenvolvidos, foi a simulação de um jato de fluido viscoelástico incidindo numa placa plana. Nas simulações realizadas com o primeiro 
método numérico foi mostrado que o parâmetro $\beta$ está intimamente ligado a viscosidade $\eta_{p}$ presente no modelo PTT. Para ambos os métodos numéricos, comparamos o comportamento do jato Newtoniano com o comportamento do jato com fluido viscoelástico seguindo a teoria de Cruickshank \& Munson [23] , [22]. Os resultados obtidos com o jato com o fluido Newtoniano estiveram de acordo com a teoria de Cruickshanck \& Munson. Entretanto, a teoria de Cruickshanck \& Munson não se aplica a fluidos viscoelásticos visto que o jato viscoelástico apresentou o fenômeno oscilante em situações em que o jato Newtoniano não apresentou o fenômeno do jato oscilante. Também, foi mostrado que o jato viscoelástico oscila devido ao aumento da viscosidade no momento em que o jato atinge a placa rígida e foi realizado um estudo onde foi mostrado que a viscoelasticidade e o número de Reynolds são fatores importantes no problema do jato oscilante. Para analisar a convergência de GENSMAC2D-PTT no problema do jato viscoelástico, foi realizado um refinamento de malha e os resultados mostraram a convergência de GENSMAC2D-PTT para o problema do jato oscilante. Os resultados numéricos apresentados neste trabalho, de ambos os métodos bidimensionais, demostraram que as técnicas numéricas desenvolvidas são capazes de simular escoamentos com superfícies livres governados pelo modelo PTT. Outro exemplo numérico de escoamentos viscoelásticos bidimensionais com superfícies livres simulados com ambos os métodos numéricos, foi a simulação do impacto de uma gota com fluido viscoelástico incidindo contra uma placa rígida plana. Aplicando o primeiro método numérico, variou-se o número de Weissenberg e observou-se a sua influência no escoamento. Enquanto que o escoamento da gota viscoelástica com $W e=1$ apresentou três fases, o escoamento da gota viscoelástica com $W e=3$ apresentou cinco fases. Ainda, comparou-se o comportamento da gota de fluido viscoelástico com o da gota de fluido Newtoniano. O último exemplo numérico de escoamentos viscoelásticos bidimensionais com superfícies livres apresentado nesta tese foi a simulação numérica do inchamento do extrudado. O primeiro método não foi capaz de simular esse problema enquanto que GENSMAC2D-PTT obteve resultados satisfatórios. Foram obtidos resultados para diversos números de Weissenberg e números de Reynolds $R e=0.1$ e $R e=1$. Os resultados obtidos com $R e=0.1$ mostraram um maior inchamento do que os resultados obtidos com $R e=1$.

O Capítulo 3 apresenta o trabalho desenvolvido para a obtenção de um método numérico para simular escoamentos viscoelásticos tridimensionais. O método GENSMAC2D-PTT foi extendido para escoamentos tridimensionais obtendo assim o método GENSMAC3D-PTT. Neste capítulo são apresentadas as equações governantes para escoamentos cartesianos tridimensionais descritos pelo modelo PTT. As condições de contorno para cada tipo de contorno, o cálculo do tensor extra-tensão no contorno rígido e as condições na superfície livre do fluido foram apresentadas em detalhes. As equações governantes são resolvidas pelo método de diferenças finitas usando uma malha diferenciada tridimensional. GENSMAC3D-PTT foi implementado no ambiente de simulação FreeFlow3D e foi utilizado para simular de escoamentos viscoelásticos tridimensionais com superfícies livres. Em particular, GENSMAC3D-PTT foi aplicado aos seguintes problemas: simulação de um jato de fluido viscoelástico incidindo numa placa rígida 
plana e simulação numérica do inchamento do extrudado. Esses resultados são originais visto que na literatura pode-se encontrar poucos resultados sobre simulação numérica de escoamentos viscoelásticos tridimensionais com superfícies livres.

Como proposta de trabalho futuro temos alguns exemplos numéricos de escoamentos de fluidos viscoelásticos que podem ser simulados pelos códigos desenvolvidos nessa tese. Dentre esses modelos podemos citar os problemas conhecidos como: contração 4:1, 'filament stretching', 'squeeze flow' e 'delayed swell'. O problema da contração 4:1 caracteriza-se pelo escoamento de um fluido através de um canal de diâmetro $D$ que passa a escoar em um canal de diâmetro menor $D_{1}=D / 4$. Neste problema estuda-se o aparecimento de vórtices no canto anterior a contração ([92], [63], [40], [95], [61]). Para que seja possível a visualização e o cálculo do comprimento dos vórtices utilizando esses métodos numéricos deve-se acrescentar uma rotina para efetuar o cálculo da função corrente. O problema do 'filament stretching' caracteriza-se pelo estiramento de um bloco de fluido que encontra-se entre duas placas paralelas ([78], [48], [88], [29], [65]). Inicialmente, as placas encontram-se em repouso e no instante $t=0$ passam a movimentar-se com uma velocidade exponencial. Para simular esse problema será necessário a implementação do movimento de fronteira rígida em função do deslocamento da placa no sistema Freeflow2D para resolver o problema bidimensional e no sistema Freeflow3D para resolver o problema tridmensional. Com estas alterações nos sistemas, acredita-se que seja possível o tratamento do 'squeeze flow' que aparece em muitas aplicações industriais. Neste problema o fluido é colocado entre duas placas e estas movimentam-se de forma a comprimir o fluido entre elas ([26], [24], [59], [49], [25]). O outro problema possível de simular é o 'delayed swell' no qual estuda-se o retardo do inchamento do extrudado. Há poucos resultados numéricos na literatura tratando deste problema ([36], [37], [41] , [17]); ainda é necessário um melhor entendimento do comportamento físico e reológico deste fenômeno. 


\section{Referências Bibliográficas}

[1] M. A. Alves, P. J. Oliveira, e F. T. Pinho, A convergent and universally bounded interpolation scheme for the treatment of advecion, International journal for numerical methods in fluids 41 (2003), 47-75.

[2] M. A. Alves, F. T. Pinho, e P. J. Oliveira, Study of steady pipe and channel flows of a single-mode Phan-Thien-Tanner fluid, Journal of Non-Newtonian Fluid Mechanics 101 (2001), 55-76.

[3] A. Amsden e F. Harlow, The SMAC method: A numerical technique for calculating incompressible fluid flows, Tech. report, Los Alamos Scientific Laboratory Report LA-4370, Los Alamos, NM, 1970.

[4] M. S. B. Araújo, Extensão de gensmac para escoamentos de fluidos governados pelos modelos integrais Maxwell and K-BKZ, Tese de doutorado, ICMC/USP, 2006.

[5] G. K. Batchelor, An introduction of fluid dynamics, Cambridge University Press, Cambridge, 1967.

[6] A. Beris, R. Armostrong, e R. Brown, Spectral finite-element calculations of the flow of a Maxwell fluid between excetric rotating cylinders, Journal of Non-Newtonian Fluid Mechanics 22 (1987), 129-167.

[7] R. B. Bird, R. C. Armstrong, e O. Hassager, Dynamics of polymetric liquids, vol. 1, Wiley, New York, 1977, Fluid Mechanics.

[8] A. Bonito, M. Picasso, e M. Laso, Numerical simulation of $3 D$ viscoelastic flows with free surfaces, Journal of Computational Physics 215 (2006), 691-716.

[9] D. Bousfield, R. Keunings, e M. Denn, Transient deformation of an inviscid inclusion in a viscoelastic extensional flow, Journal of Non-Newtonian Fluid Mechanics 27 (1988), $205-221$. 
[10] D. Bousfield, R. Keunings, G. Marruci, e M. Denn, Nonlinear analysis of the surface tension driven breakup of viscoelastic filaments, Journal of Non-Newtonian Fluid Mechanics 21 (1986), 79-97.

[11] E. Brasseur, M. Fyrillas, G. Georgiou, e M. Crochet, The time-dependent extrudate-swell problem of an Oldroyd-B fluid with slip along the wall, Journal of Rheology 42 (1994), 549-566.

[12] R. E. S. Bretas e M. D'Avila, Reologia de polímeros fundidos, editora Ufscar, São Carlos, 2000.

[13] E. O. A. Carew, P. Townsed, e M. F. Webster, A Taylor-Petrov-Galerkin algorithm for viscoelastic flow, Journal of Non-Newtonian Fluid Mechanics 50 (1993), 253-287.

[14] D. M. Carvalho, Desenvolvimento de um sistema de simulação de escoamentos de fluidos com superficies livres bidimensionais, Dissertação de mestrado, ICMC/USP, 2004.

[15] A. Castelo, M. F. Tomé, C.N.L. César, S. McKee, e J.A. Cuminato, Freeflow: An integrated simulation system for three-dimensional free surface flows, Journal of Computating and Visualization in Science 2 (2000), 199-210.

[16] A. Castelo, M. F. Tomé, S. McKee, e J. A. Cuminato, Freeflow: An integrated simulation system for three-dimensional free surface flows, Computing and Visualization in Science 2 (2000), 199-210.

[17] M. Cloitre, T. Hall, C. Mata, e D. D. Joseph, Delayed-die swell and sedimentation of elongated particles in wormlike micellar solutions, Journal of Non-Newtonian Fluid Mechanics 79 (1998), 157-171.

[18] J. Cormenzana, A. Ledda, e B. Debbaut, Calculation of free surface flows using connfessitt, Journal of Rheology 45 (2001), 237-258.

[19] A. B. Costacurta, Estratégias 'upwind' and modelagem k-epsilon para simulação numérica de escoamentos com superfícies livres em altos números de reynolds, Dissertação de mestrado, ICMC/USP, 2005.

[20] M. J. Crochet, B. Debbaut, R. Keunings, e J. M. Marchal, Polyflow: a multi-purpose finite element program for continuous polymer flows, K. T. O’Brien (Ed.), Applications of CAE in Extrusion and other Continuous Processes, 1992.

[21] M. J. Crochet e R. Keunings, Finite element analysis of die-swell of a highly elastic fluid, Journal of Non-Newtonian Fluid Mechanics 10 (1982), 339-356.

[22] J. O. Cruickshank, Low-Reynolds-number instabilities in stagnating jet flows, Journal of Fluid Mechanics 193 (1988), 111-127. 
[23] J. O. Cruickshank e B. R. Munson, Viscous-fluid buckling of plane axisymmetric jets, Journal of Fluid Mechanics 113 (1981), 221-239.

[24] B. Debbaut, Non-isothermal and viscoelastic effects in the squeeze flow between infinite plates, Journal of Non-Newtonian Fluid Mechanics 98 (2001), 15-31.

[25] B. Debbaut e K. Thomas, Simulation and analysis of oscillatory squeeze flow, Journal of Non-Newtonian Fluid Mechanics 124 (2004), 77-91.

[26] J. Engmann, C. Servais, e A. S. Burbidge, Squeeze flow theory and applications to rheometry: A review, Journal of Non-Newtonian Fluid Mechanics 132 (2005), 1-27.

[27] X. Fan, N. Phan-Thien, e R. Zheng, A direct simulation of fibre suspensions, Journal of Non-Newtonian Fluid Mechanics 74 (1998), 113-135.

[28] V. G. Ferreira, Análise and implementação de esquemas de convecção e modelos de turbulência para a simulação de escoamentos incompressiveis envolvendo superfícies livres, Tese de doutorado, ICMC/USP, 2001.

[29] Katerina Foteinopoulou, Vlasis G. Mavrantzas, e John Tsamopoulos, Numerical simulation of bubble growth in newtonian and viscoelastic filaments undergoing stretching, Journal of Non-Newtonian Fluid Mechanics 122 (2004), 177-200.

[30] M. Griebel, D. Thomas, e T. Neunhoeffer, Numerical simulation in fluid dynamics. a practical introduction, Society for Indrustrial and Applied Mathematics, 1997.

[31] L. Grossi, Solução numérica de escoamentos axissimétricos não-newtonianos com superfícies, Dissertação de mestrado, ICMC/USP, 1997.

[32] L. Grossi, Desenvolvimento de métodos numéricos para a simulação de escoametos não-newtonianos and viscoelásticos com superfícies livres, Tese de doutorado, ICMC/USP, 2003.

[33] F. H. Harlow e J. E. Welch, The mac method, Physics of Fluids 8 (1965), 2182-2189.

[34] X. Huang, N. Phan-Thien, e R. I. Tanner, Viscoelastic flow between eccentric rotating cylinders: Unstructured control volume method, Journal of Non-Newtonian Fluid Mechanics 64 (1996), 71-92.

[35] M. A. Hulsen, E. A. J. F. Peters, e B. H. A. A. van den Brule, A new approach to the deformation fields method for solving complex flows using integral constitutive equations, J. Non-Newt. Fluid Mech. 98 (2001), 201-221.

[36] D. D. Joseph e E. M. Chen, Delayed die swell, Journal of Non-Newtonian Fluid Mechanics 24 (1987), 31-65. 
[37] D. D. Joseph e C. Christodoulou, Independent confirmation that delayed die swell is a hyperbolic transition, Journal of Non-Newtonian Fluid Mechanics 48 (1993), 225-235.

[38] R. Keunings, An algorithm for the simulation of transient viscoelastic flows with free surfaces, Journal of Computational Physics 62 (1986), 199-220.

[39] R. Keunings e D. Bousfield, Analysis of surface tension driven leveling in horizontal viscoelastic films, Journal of Non-Newtonian Fluid Mechanics 22 (1987), 219-233.

[40] R. Keunings e M. J. Crochet, Numerical simulation of the flows of a viscoelastic fluid through an abrupt contraction, Journal of Non-Newtonian Fluid Mechanics 14 (1984), 279-299.

[41] D. G. Kiriakidis, H. J. Park, E. Mitsoulis, B. Vergnes, e J. F. Agassant, A study of stress distribution in contraction flows of anb lldpe melt, Journal of Non-Newtonian Fluid Mechanics 47 (1993), 339-356.

[42] M. Kolte, H. Rasmussen, e O. Hassager, Transient filament stretching rheometer.2. numerical simulation, Rheology Acta 36 (1997), 285-302.

[43] X. L. Luo, Numerical simulation of Weissenberg phenomena - the rod-climbing of viscoelastic fluids, Computer Methods in Applied Mechanics and Engineering 180 (1999), 393-412.

[44] X. L. Luo e R. I. Tanner, A streamline element scheme for solving viscoelastic flow problems part II : Integral constitutive models, J. Non-Newtonian Fluid Mech. 22 (1986), 61-89.

[45] X. L. Luo e R. I. Tanner, Finite element simulation of long and short circular die extrusion experiments using integral models, International Journal for Numerical Methods in Engineering 25 (1988), 9-22.

[46] C. W. Macosko, Rheology: principles, measurements and applications, VHC, 1994.

[47] J. M. Marchal e M. J. Crochet, A new mixed finite element for calculating viscoelastic flow, Journal of Non-Newtonian Fluid Mechanics 26 (1987), 77-114.

[48] H. Matallah, M. J. Banaai, K. S. Sujatha, e M. F. Webster, Modelling filament stretching flows with strain-hardening models and sub-cell approximations, Journal of Non-Newtonian Fluid Mechanics 134 (2006), 77-104.

[49] Y. Mochimaru, Fast squeezing flow of viscoelastic fluids, Journal of Non-Newtonian Fluid Mechanics 9 (1987), 157-178.

[50] G. Mompean e M. Deville, Unsteady finite volume of Oldroyd-B fluid through a three-dimensional planar contraction, Journal of Non-Newtonian Fluid Mechanics $\mathbf{7 2}$ (1997), 253-279. 
[51] C. M. Oishi, Análise and implementação de métodos implícitos no sistema Freeflow2D, Dissertação de mestrado, ICMC/USP, 2004.

[52] J. Oliveira, Desenvolvimento de um sistema de simulação de escoamentos de fluidos com superficies livres bidimensionais, Dissertação de mestrado, ICMC/USP, 1999.

[53] M. L. B. Oliveira, Freeflow-Axi: Um ambiente de simulação de escoamentos axissimétricos com superfícies livres, Dissertação de mestrado, ICMC/USP, 2002.

[54] P. J. Oliveira e F. T. Pinho, Analytical solution for fully developed channel and pipe flow of Phan-Thien-Tanner fluids, Journal of Fluid Mechanics 387 (1999), 271-280.

[55] P. Olley, An adaptation of the separable KBKZ equation for comparable response in planar and axisymmetric flow, J. Non-Newtonian Fluid Mech. 95 (2000), 35-53.

[56] P. Olley, R. Spares, e P. D. Coates, A method for implementing time-integral constitutive equations in commercial CFD packages, J. Non-Newtonian Fluid Mech. 86 (1999), 337-357.

[57] L. S. Paiva, Desenvolvimento de um modelador de movimentos para o sistema Freeflow3D, Dissertação de mestrado, ICMC/USP, 2000.

[58] N. Phan-Thien, A nonlinear network viscoelastic model, Journal of Rheology 22 (1978), no. 3, 259-283.

[59] N. Phan-Thien e H. T. Low, Squeeze-film flow of a viscoelastic fluid a lubrication model, Journal of Non-Newtonian Fluid Mechanics 28 (1988), 129-148.

[60] N. Phan-Thien e R. I. Tanner, A new constitutive equation derived from network theory, Journal of Non-Newtonian Fluid Mechanics 2 (1977), 353-365.

[61] T. N. Phillips e A. Williams, Viscoelastic flow though a planar contraction using a semi-lagrangian finite volume method, Journal of Non-Newtonian Fluid Mechanics 87 (1999), 215-246.

[62] T. N. Phillips e A. Williams, Comparison of creeping and inertial flow of an Oldroyd-B fluid though a planar and axisymmetric contraction, Journal of Non-Newtonian Fluid Mechanics 108 (2002), 25-47.

[63] F. T. Pinho, M. A. Alves, e P. J. Oliveira, Benchmark solutions for the flow of Oldroyd-B and ptt fluids in planar contractions, Journal of Non-Newtonian Fluid Mechanics 110 (2003), 45-75.

[64] D. Rajagopalan, R. C. Armstrong, e R. A. Brown, Finite element methods for calculation of steady viscoelastic flow using constitutive equations with a newtonian viscosity, Journal of Non-Newtonian Fluid Mechanics 36 (1990), 159-192. 
[65] H. K. Rasmussen e O. Hassager, Three-dimensional simulations of viscoelastic instability in polymeric filaments, Journal of Non-Newtonian Fluid Mechanics 82 (1999), 189-202.

[66] N. M. Ribe, Periodic folding of viscous jets, Physical Review E 68 (2003), Art. No. 036305 Part 2.

[67] N. M. Ribe, Coiling of viscous jets, Proceedings of the Royal Society of London Series A - Mathematical Physical and Engineering Sciences 460 (2004), 3223-3239.

[68] M. E. Ryan e A. Dutta, A finite difference simulation of extrudate swell, Proceedings of Second World Congress of Chemical Engineering, 1981, pp. 277-281.

[69] M. H. Sabatini, Expansão do sistema Freeflow3D para escoamentos com influência da temperatura, Dissertação de mestrado, ICMC/USP, 2002.

[70] F. L. P. Santos, Simulação numérica de escoamentos multifásicos utilizando o sistema Freeflow2D, Dissertação de mestrado, ICMC/USP, 2001.

[71] W. Shyy, H. S. Udaykumar, M. M. Rao, e R. W. Smith, Computational fluid dynamics with moving boundaries, Hemisphere, 1996.

[72] G. F. Silva, Análise and implementação de modelos viscoelásticos no sistema Freeflow2D, Dissertação de mestrado, ICMC/USP, 2003.

[73] J. M. Silva, Simulação numérica de escoamentos com superfícies livres e com influência da temperatura, Dissertação de mestrado, ICMC/USP, 2002.

[74] M. A. Silva, Desenvolvimento de um método numérico para simular escoamentos viscoelásticos axissimétricos com superfícies livres, Dissertação de mestrado, ICMC/USP, 2005.

[75] R. S. Siquieri, Análise and implementação de modelos não-newtonianos no sistema Freeflow2D, Dissertação de mestrado, ICMC/USP, 2002.

[76] F. S. Sousa, Simulação de escoamentos multifásicos usando o sistema Freeflow3D, Dissertação de mestrado, ICMC/USP, 2002.

[77] R. I. Tanner, A theory of die-swell, Journal of Polymer Science 8 (1970), 2067-2078.

[78] V. Tirtaatmadja e T. Sridhar, A filament stretching device for measurement of extensional viscosity, Journal of Rheology 37 (1993), 1081-1102.

[79] L. Tomé, M. F. Grossi, A. Castelo, J. A. Cuminato, N. Mangiavacchi, V. G. Ferreira, F. S. Sousa, e S. McKee, A numerical method for solving three-dimensional generalized newtonian free surface flows, Journal of Non-Newtonian Fluid Mechanics 123 (2004), 85-103. 
[80] M. F. Tomé, A. Castelo, J. A. Cuminato, N. Mangiavacchi, e S. McKee, GENSMAC3D: A numerical method for solving unsteady three-dimensional free surface flows, International Journal for Numerical Methods in Fluids 37 (2001), 747-796.

[81] M. F. Tomé, A. Castelo, F. Federson, e J. A. Cuminato, A numerical method for solving the Oldroyd-B model for $3 D$ free surface flows, XIV Congreso sobre Metodos Numericos Y Sus Aplicaciones, ENIEF 2004 (San Carlos de Bariloche, Argentina) (CD-ROM, ed.), 2004.

[82] M. F. Tomé, A. Castelo, e V. G. Ferreira, Numerical solution of the Oldroyd-B model for three-dimensional viscoelastic free surface flows, European Conference on Computational Fluid Dynamics - ECCMAS CFD (TU Delft, The Netherlands) (P. Wesseling, E. Oñate, e J. Périaux, eds.), 2006.

[83] M. F. Tomé, J. L. Doricio, A. Castelo, J. A. Cuminato, e S. McKee, Solving viscoelastic free surface flow of a second order fluid using a Marker-and-Cell approach, International Journal for Numerical Methods in Fluids - artigo aceito para publicação. (2006).

[84] M. F. Tomé, N. Mangiavacchi, A. Castelo, J. A. Cuminato, e S. McKee, A finite difference techique for simulating unsteady viscoelastic free surface flows, Journal of Non-Newtonian Fluid Mechanics 106 (2002), 61-106.

[85] M. F. Tomé e S. McKee, GENSMAC: a computational Marker-and-Cell method for free surface flows in general domains, Journal of Computational Physics 110 (1994), 171-186.

[86] M. F. Tomé e S. McKee, Numerical simulation of viscous flow: Buckling of planar jets, International Journal for Numerical Methods in Fluids 29 (1999), 705-718.

[87] M. F. Tomé, S. McKee, L. Barrat, D. A. Jarvis, e A. J. Patrick, An experimental and numerical investigation of container filling with viscous liquids, International Journal for Numerical Methods in Fluids 31 (1999), 1333-1353.

[88] M. F. Webster, H. Matallah, e K. S. Sujatha, Sub-cell approximations for viscoelastic flows-filament stretching, Journal of Non-Newtonian Fluid Mechanics 126 (2005), 187-205.

[89] J. E. Welch, F. H. Harlow, J. P. Shannon, e B. J. Daly, The MAC method, Tech. report, Los Alamos Scientific Laboratory Report LA-3425, Los Alamos, 1966.

[90] A. M. Worthington, A study of splashes, London:Longmans, Green, 1967.

[91] S. C. Xue, N. Phan-Thien, e R. I. Tanner, Numerical study of secondary flows of viscoelastic fluid in straight pipes by an implicit finite volume method, Journal of Non-Newtonian Fluid Mechanics 59 (1995), 191-213. 
[92] S. C. Xue, N. Phan-Thien, e R. I. Tanner, Three-dimensional numerical simulation of viscoelastic flows through planar contractions, Journal of Non-Newtonian Fluid Mechanics 74 (1998), 195-245.

[93] S. C. Xue, R. I. Tanner, e N. Phan-Thien, Three-dimensional numerical simulation of viscoelastic flows - predictability and accuracy, Computer Methods in Applied Mechanics and Engineering 180 (1999), 305-331.

[94] M. W. Yao e G. McKinley, Numerical simulation of extensional deformations of viscoelastic liquid bridges in filaments stretching devices, Journal of Non-Newtonian Fluid Mechanics 74 (1998), 47-88.

[95] J. Yoo e Y. Na, A numerical study of the planar contraction flow of a viscoelastic fluid using the simpler algorithm, Journal of Non-Newtonian Fluid Mechanics 30 (1991), 89-106. 